\title{
Systematic Design of \\ Sigma-Delta Analog-To-Digital \\ CONVERTERS
}




\section{Systematic Design of Sigma-Delta Analog-TO-Digital CONVERTERS}

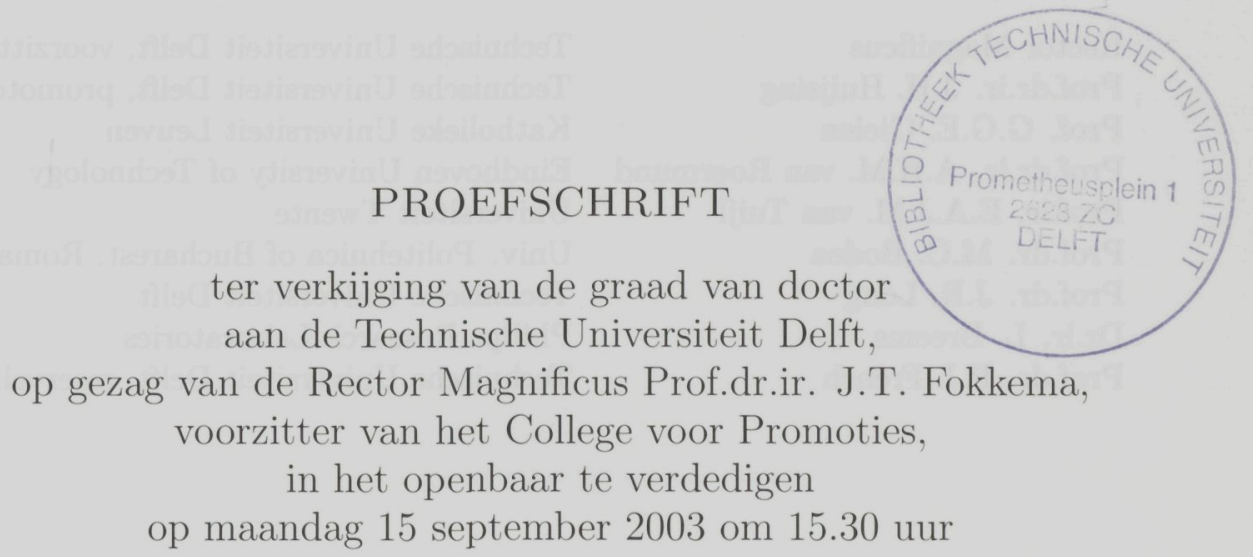

door

Ovidiu BAJDECHI

inginer

Facultatea de Electronică şi Telecomunicaţii - Bucureşti geboren te Constanţa, Roemenië 
Dit proefschrift is goedgekeurd door de promotor:

Prof.dr.ir. J.H. Huijsing

Samenstelling promotiecommissie:

Rector Magnificus

Prof.dr.ir. J.H. Huijsing

Prof. G.G.E. Gielen

Prof.dr.ir. A.H.M. van Roermund

Prof.ir. E.A.J.M. van Tuijl

Prof.dr. M.C. Bodea

Prof.dr. J.R. Long

Dr.ir. L. Breems

Prof.dr. P.J. French
Technische Universiteit Delft, voorzitter Technische Universiteit Delft, promotor Katholieke Universiteit Leuven

Eindhoven University of Technology Universiteit Twente

Univ. Politehnica of Bucharest, Romania Technische Universiteit Delft Philips Research Laboratories Technische Universiteit Delft, reservelid

Printed by [OPTIMA] Grafische Communicatie, Rotterdam

ISBN: 90-6734-355-2

Copyright (C)2003 by O. Bajdechi

All rights reserved. No part of this publication may be reproduced or distributed in any form or by any means, or stored in a database or retrieval system, without the prior written permission of the author.

Printed in The Netherlands 
To my wife, Simona Maria 


\section{Table of Contents}

1 Introduction

1.1 Analog-to-Digital Conversion.

From Nyquist to Sigma-Delta . . . . . . . . . . . . . . . . 1

1.1.1 Nyquist-rate ADCs . . . . . . . . . . . . . . . . 2

1.1 .2 Oversampled ADCs ................... 3

1.1 .3 Noise Shaping ADCs . . . . . . . . . . . . . . . . . . 4

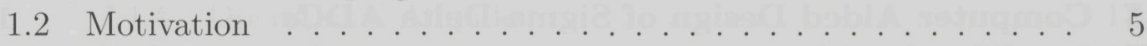

1.3 Thesis Organization . . . . . . . . . . . . . 6

2 Architecture-Level Analysis of Sigma-Delta ADCs 9

2.1 Principle and Operation ................... 9

2.1.1 Linear Model. Transfer Functions . . . . . . . . . . . . . . . . 9

2.1.2 Non-Linear Loop Transfer . . . . . . . . . . . . . . . . . . 12

2.1.3 Performance Metrics . . . . . . . . . . . . . . . . . . 14

2.2 Sigma-Delta ADCs with Discrete-Time Loop Filters . . . . . . . 16

2.2.1 Design of Loop Transfer Functions . . . . . . . . . . . . 16

2.2 .2 Single Loop Topologies . . . . . . . . . . . . . . . . . . . . 19

2.2 .3 Cascaded Topologies . . . . . . . . . . . . . . . 26

2.3 Sigma-Delta ADCs with Continuous-Time Loop Filters . . . . . 31

2.3.1 Equivalence Between Discrete-Time and Continuous-Time Loop Filters . . . . . . . . . . . . . . . . . . . . . 32

2.3.2 Effects of DAC Waveform Shape ... . . . . . . . . . 33

2.3.3 Single Loop Topologies . . . . . . . . . . . . . . . . . . . . . . . . . . . . . . . 38

2.3.4 Cascaded Topologies . . . . . . . . . . . . . 40

3 Discrete-Time Circuit Design $\quad 45$

3.1 Switched-Capacitor Integrators . . . . . . . . . . . . . 48

3.1.1 Topologies for Summing Integrators . . . . . . . . . . . 48

3.1 .2 Effects of Circuit Non-idealities . . . . . . . . . . . . . 51

3.1 .3 Clocking Signals . . . . . . . . . . . . . . . . . . . 59

3.1 .4 Operational Amplifier Topologies . . . . . . . . . . 63 
3.1.5 Power Consumption Analysis . . . . . . . . . . . . . 66

3.2 Switched-Capacitor Amplifiers . . . . . . . . . . . . . . . 69

3.3 Quantizer ......................... 71

3.3 .1 The Dynamic Comparator . . . . . . . . . . . . 71

3.3 .2 Multibit Quantizers . . . . . . . . . . . . . 72

3.4 Multibit, SC Digital-to-Analog Converters . . . . . . . . . . 73

4 Continuous-Time Circuit Design $\quad 79$

4.1 Active RC Integrator . . . . . . . . . . . . . . . . . . . . . . . 81

4.1 .1 Topology . . . . . . . . . . . . . . . . 81

4.1 .2 Effects of Circuit Non-Idealities . . . . . . . . . . . . . . 84

4.1.3 Operational Amplifier Topologies . . . . . . . . . . . . . . 91

4.1.4 Power Consumption Analysis . . . . . . . . . . . . . . . 93

4.2 Quantizer ............................. 96

4.3 Digital-to-Analog Converters . . . . . . . . . . . . . . . . . . . . . 97

4.3 .1 Return-to-Zero DACs . . . . . . . . . . . . . . 97

4.3 .2 Calibrated Multibit DACs . . . . . . . . . . . . . 99

5 Computer Aided Design of Sigma-Delta ADCs 103

5.1 Filter-Level Design . . . . . . . . . . . . . . . . . . 103

5.1 .1 Design of Stable Discrete-Time NTF . . . . . . . . . . . 105

5.1 .2 Dynamic Range Estimation . . . . . . . . . . . . . . . 108

5.1 .3 Mapping DT TF to CT TF . . . . . . . . . . . 111

5.2 Architecture-Level Design . . . . . . . . . . . . . . . . . . 115

5.2 .1 Design Space Exploration . . . . . . . . . . . 115

5.2 .2 Optimization of Loop Gains . . . . . . . . . . . . . 117

5.2 .3 Performance Test . . . . . . . . . . . . . . 120

5.2.4 Estimation of Power Consumption . . . . . . . . . . . 121

5.2 .5 Yield Analysis and Optimization . . . . . . . . . . . . 122

5.3 Design Examples . . . . . . . . . . . . . . . . . . . . 123

5.3 .1 Audio Sigma-Delta ADC . . . . . . . . . . . . 124

5.3.2 Delta-Sigma ADC for xDSL Applications . . . . . . . 125

6 Sigma-Delta ADC for Audio Applications 129

6.1 The Electret Microphone . . . . . . . . . . . . . . . . . 130

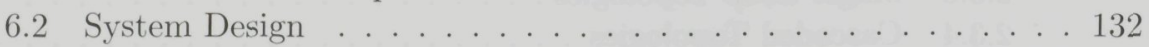

6.2 .1 Project Specifications . . . . . . . . . . . . 132

6.2.2 Switched-Capacitor vs. Continuous-Time Loop Filter . . 133

6.3 Sigma-Delta ADC Design . . . . . . . . . . . . . . . . . 134

6.3 .1 Topology ... . . . . . . . . . . . . . . . 134

6.3 .2 NTF and STF Design . . . . . . . . . . . . 135

6.3.3 High Input Impedance Continuous-Time Integrator . . . . 136

6.3.4 Switched-Capacitor Integrators . . . . . . . . . . . . 141

vi Systematic Design of $\Sigma \Delta$ ADCs 
6.3.5 Adder and Comparator . . . . . . . . . . . . . . . 142

6.3.6 Timing of Feedback Signals . . . . . . . . . . . . . . . 143

6.4 Linearity Analysis . . . . . . . . . . . . . . . . . . . . . . 144

6.5 Experimental Results . . . . . . . . . . . . . . 146

7 Broadband, High Dynamic Range Sigma-Delta ADC 153

7.1 Project Specifications . . . . . . . . . . . . . . . 155

7.2 CAD-Assisted Design Optimization . . . . . . . . . . . 157

7.2.1 Directing the CAD Software Toward an Optimal Solution 157

7.2 .2 Loop Coefficients . . . . . . . . . . . . . . . . 162

7.2.3 Power Dissipation for DT and CT Circuits . . . . . . . 164

7.3 Design of the 16-bit CT Integrator . . . . . . . . . . 166

7.3 .1 Integrator Topology . . . . . . . . . . . 166

7.3.2 Operational Amplifier . . . . . . . . . . . . . . 168

7.4 Design of the 14-bit SC Integrator . . . . . . . . . . . . . 171

7.4 .1 Integrator Topology . . . . . . . . . . . . . . . . 171

7.4 .2 Linearized Sampling Switch . . . . . . . . . . . . . . . 172

7.4 .3 Capacitor Sizing . . . . . . . . . . . . . . . . . . . . . . 174

7.4 .4 Operational Amplifier . . . . . . . . . . . . . . . 174

7.5 Higher Order Integrators . . . . . . . . . . . . . . . . . 177

7.5.1 Integrator Topology . . . . . . . . . . . . . . . 177

7.5.2 Capacitors Sizing and Opamps Performance . . . . . . . . 178

7.6 Feed-Forwards Adder and 31-Levels Quantizer . . . . . . . . . 178

7.6 .1 Zero-Delay SC Adder . . . . . . . . . . . . . . . 178

7.6.2 Quantizer Topology . . . . . . . . . . . . . . 179

7.7 Calibration of Current-Mode CT DAC . . . . . . . . . . . . . . 181

7.7.1 DAC Matching Requirements . . . . . . . . . . . . . . 181

7.7.2 CT Current-Mode DAC . . . . . . . . . . . . . . 183

7.7 .3 Active Calibration . . . . . . . . . . . . . . . . . 185

7.7 .4 Calibration DAC . . . . . . . . . . . . . . . . . 189

7.8 Transistor-Level Simulation Results . . . . . . . . . . . . . . . 190 
viii Systematic Design of $\Sigma \Delta$ ADCs 


\section{List of Abbreviations and Symbols}

\section{Abbreviations}

A/D analog-to-digital

ADC analog-to-digital converter

ADSL asymmetric digital subscriber line

BER bit error rate

BW bandwidth

CAD computer-aided design

CMC common-mode control

CMOS complementary metal oxide semiconductor

CMRR common-mode rejection ratio

Codec coder-decoder

CT continuous-time

D/A digital-to-analog

DAC digital-to-analog converter

DR dynamic range

DSL digital subscriber line

DSP digital signal processor

DT discrete time

ENOB effective number of bits

FDD frequency-division duplexing

FDM frequency-division multiplexing

FFT fast Fourier transform

FOM figure of merit

JFET junction field-effect transistor

MOS metal oxide semiconductor

NMOS N-channel MOS transistor

NRZ non-return to zero

NTF noise transfer function

Opamp operational amplifier 
PAM pulse amplitude modulation

PMOS P-channel MOS transistor

POTS plain old telephone service

RC resistor-capacitor

RTZ return to zero

$\mathrm{Rx}$ receive

OSR oversampling ratio

$\Sigma \Delta \quad$ sigma-delta

$\mathrm{S} / \mathrm{H} \quad$ sample and hold

SNDR signal-to-(noise plus distortion) ratio

SC switched-capacitor

STF signal transfer function

THD total harmonic distortion

Tx transmit

UGB unity-gain bandwidth

$\mathrm{V} / \mathrm{I} \quad$ voltage-to-current 


\section{Symbols}

$A_{D C} \quad$ opamp voltage gain at DC

BITS number of quantizer bits

C capacitance

$\Delta \quad$ (large) variation

$D_{i} \quad$ input-referred integrator distortion

$E(z) \quad$ quantization noise

$f$ frequency

$f_{b} \quad$ signal bandwidth

$f_{s} \quad$ sampling frequency

$G_{m} \quad$ generic transconductance

$\gamma$

$g_{m} \quad$ transistor (pair) transconductance

HD3 third-order harmonic distortion

$H(s) \quad$ continuous-time transfer function

$H(z) \quad$ discrete-time transfer function

I current

$J \quad \sqrt{-1}$

$k \quad$ Boltzmann's constant

$L \quad$ channel length for MOS transistors

LOOPS number of cascaded loops

$\mu \quad$ carrier mobility

$N \quad$ number of quantizer bits

$\omega \quad$ frequency

ORDER order of $\Sigma \Delta$ loop

$P_{i} \quad$ input-referred integrator noise power

$P_{n} \quad$ noise power

$Q_{n} \quad$ in-band quantization noise power

$R \quad$ resistance

$s \quad$ Laplace operator

$\sigma \quad$ standard deviation

$S_{n} \quad$ spectral power density (of noise)

$T$ temperature

$T_{s} \quad$ sampling period

$V \quad$ voltage

$V_{d s} \quad$ drain-source voltage of a MOS transistor

$V_{g s} \quad$ gate-source voltage of a MOS transistor

$V_{\text {ref }} \quad$ reference voltage

$V_{T} \quad$ threshold voltage of a MOS transistor

$W \quad$ channel width for a MOS transistor

z $\quad$ z-domain frequency 
xii Systematic Design of $\Sigma \Delta$ ADCs 


\section{1}

\section{Introduction}

\subsection{Analog-to-Digital Conversion. From Nyquist to Sigma-Delta}

Ever since the analog computers have been ruled out as a high-performance, low-cost option to implement programmable, complex control loops [1], the need arose for faster, more and more accurate interfaces between the analog world and the digital processors acting as the decision element of the system. Digital processors are easily programmable and highly integrable, densities of millions of logic gates per chip being the norm these days. With a central digital core to perform the bulk of processing and analog interfaces to connect to its environment, the mixed-signal systems are present in appliances ranging from mobile remote controls to multi-Gbit/s transceivers.

As the "brain" of almost any control/communications system, the digital processor connects to the external world through analog-to-digital converters (ADCs) for inbound analog data and digital-to-analog converters (DACs) for outbound analog signals. Because all ADCs contain inside at least one DAC placed in a control/comparison loop [2], the ADC can only be as good as the $\mathrm{DAC}$ is and, therefore, the ADC becomes the most challenging interface block. One of the most important design challenges is to integrate the ADC on the same chip with the digital processor using a digital (typically CMOS) technology offering fast transistors biased at low voltage. The tendency is therefore to use ADC architectures which trade accuracy for speed, faster analog designs needing less-complex and lower-performance component blocks to attain a given conversion accuracy compared to their slower counterparts.

The technology sets the upper limit for the switching speed however, so the trade-off speed-accuracy must be applied both ways to find the best fitting architecture. Different architectures are used for different analog signal 


\section{Introduction}

bandwidths and different available technologies. Nyquist-rate ADCs are used for high-bandwidth conversion while oversampling, noise-shaping $\Sigma \Delta$ ADCs are used for low- and intermediate-frequency signals, their range of applicability extending with faster switching speeds available.

\subsubsection{Nyquist-rate ADCs}

Analog signals are continuous both in time and amplitude and their spectrum contains non-zero tones in a finite frequency band as an effect of their continuity in amplitude. In Fig. 1.1 the input signal spectrum is placed around DC and extends to $f_{b}$ in the positive spectrum. The analog-to-digital conversion requires the analog input signal $V_{i}(t)$ to firstly be sampled by a sample-and-hold (the block $S / H$ ) which transforms it into an analog, discrete-time signal, only changing its amplitude at periodic intervals $T_{s}$ (set by the sampling frequency $\left.f_{s}, T_{s}=1 / f_{s}\right)$. Because sampling introduces instantaneous amplitude changes in the analog signal, the spectrum of the sampled signal has infinite bandwidth, by replicating the input signal spectrum (the void trapezoid at DC in Fig. 1.1) around the multiples of the sampling frequency (shaded spectra around $\left.f_{s}, 2 f_{s} \cdots\right)$. The name Nyquist-rate of this architecture comes from the sampling theorem also known as the Nyquist theorem stating that $f_{s}$ should be at least two times larger than $f_{b}$,

$$
f_{s} \geq 2 f_{b}
$$

for error-free reconstruction of the input signal. Sampling with frequencies lower than $2 f_{b}$ introduces aliasing which changes the image of input signal spectrum in the sampled signal. Aliasing defines the overlapping of the input signal spectrum with the first replica of itself introduced by sampling at $2 f_{s}$.

Sampling is required to hold a constant signal $V_{s}(t)$ at the input of the $A D C$ block while the amplitude is quantized as an integer/integer ratio related to the

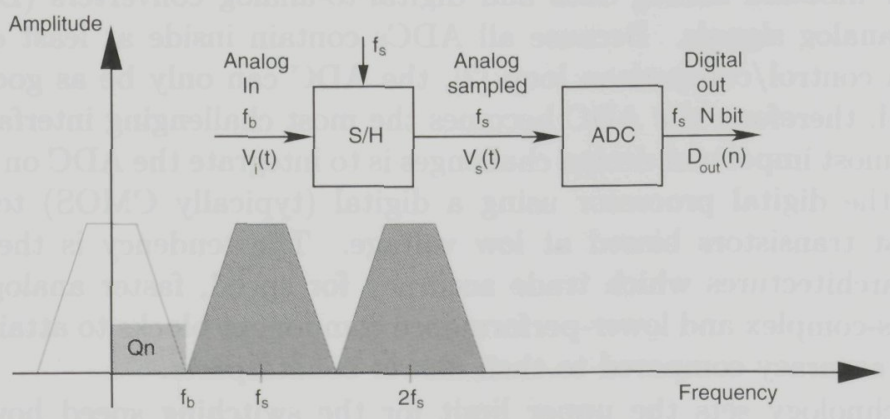

Figure 1.1: Nyquist-rate $A D C$ 


\subsection{Analog-to-Digital Conversion.From Nyquist to Sigma-Delta}

reference level (typically a voltage) $V_{\text {ref }}$,

$$
\frac{V_{s}}{V_{\text {ref }}}=\frac{D_{\text {out }}}{2^{N}-1}
$$

if the ADC has $N$ bits of resolution.

From a "spectral" point of view, the quantization introduces quantization noise in the signal bandwidth which limits the conversion resolution (as the ratio between $V_{\text {ref }}$ and the minimal signal that can be converted). Assuming the error lays with equal probability between two adjacent quantization levels spaced at $V_{\text {ref }} /\left(2^{N}-1\right)$, the quantization noise has a white spectrum (shaded rectangle marked $Q_{n}$ in Fig. 1.1) and its root mean square (rms) power is [3]

$$
e_{r m s}^{2}=\frac{V_{r e f}^{2}}{12\left(2^{N}-1\right)^{2}}
$$

For $f_{s}=2 f_{b}$ the entire quantization noise power is equally distributed from DC to $f_{s} / 2=f_{b}$, hence $e_{r m s}^{2}$ is all found inside the signal bandwidth,

$$
Q_{n}=e_{r m s}^{2}
$$

This makes the conversion resolution to be equal with the number of bits in the ADC. An increase in resolution can be attained by increasing the number of ADC bits, a method not always convenient since the complexity of the ADC is exponentially increasing with its number of bits.

\subsubsection{Oversampled ADCs}

If an ADC operates faster than $2 f_{b}$, an oversampled $A D C$ is built (Fig. 1.2). In this case, the oversampling ratio (OSR) is a design parameter showing how

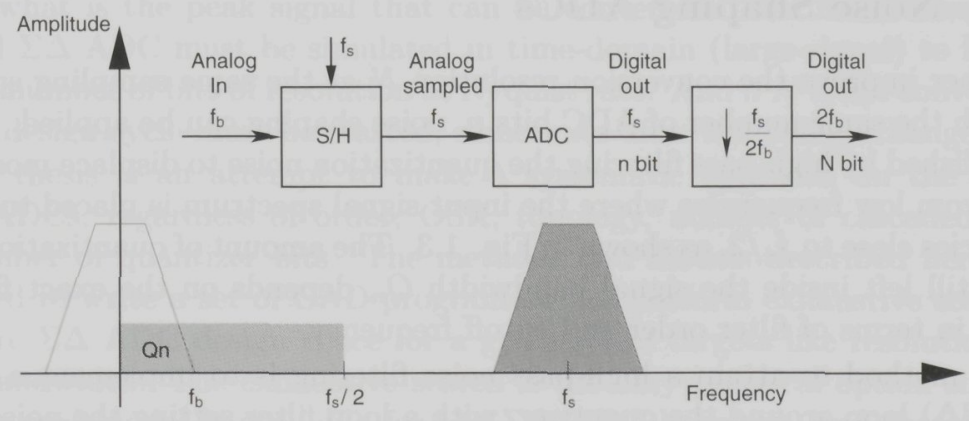

Figure 1.2: Oversampling $A D C$ 
many times is $f_{s}$ larger than the minimal value required by the Nyquist theorem

$$
O S R=\frac{f_{s}}{2 f_{b}}
$$

The ADC with $\mathrm{N}$ bits of resolution adds the same quantization noise power (Eq. 1.3) to the quantized signal. The difference is that only part of the quantization noise power falls in the signal bandwidth since the bandwidth of the quantization noise ranges now from DC to $f_{s} / 2$,

$$
Q_{n}=\frac{e_{r m s}^{2}}{f_{s} / 2} f_{b}=\frac{e_{r m s}^{2}}{O S R}
$$

The resolution is therefore increased by 1 bit (four times less noise power from DC to $f_{b} / 2$ ) for 4 times increase in OSR.

In practice the oversampling is mostly used with a different approach, illustrated in Fig. 1.2. Knowing the analog signal bandwidth $f_{b}$ and the ADC sampling frequency $f_{s}$, a number of bits $n$ smaller than the final target resolution $N$ is implemented to reduce ADC complexity, given a technology-limited OSR. By every 4 times increase in OSR the difference between $N$ and $n$ is increased by 1 bit. After the ADC, a digital decimation filter reduces the sampling rate to $2 f_{b}$ while increasing the resolution to $N$ bits and rejecting the quantization noise between $f_{b}$ and $f_{s} / 2$.

For large OSRs the $S / H$ block is not really required since the input signal changes little during one $T_{s}$ period. Given enough OSR, the ADC resolution $n$ can eventually be reduced to 1 bit. The OSR is upper limited by the technology used to implement the ADC (which limits its decision speed $f_{s}$ ), so for large resolutions and speeds 1 bit ADCs may still not be enough.

\subsubsection{Noise Shaping ADCs}

To further improve the conversion resolution $N$ at the same sampling speed $f_{s}$ and with the same number of ADC bits $n$, noise shaping can be applied. This is accomplished by high-pass filtering the quantization noise to displace most of its power from low frequencies where the input signal spectrum is placed to higher frequencies close to $f_{s} / 2$, as shown in Fig. 1.3. The amount of quantization noise power still left inside the signal bandwidth $Q_{n}$ depends on the exact filtering applied in terms of filter order and cutoff frequency.

One method to attain a high-pass noise filtering is to implement a sigmadelta $(\Sigma \Delta)$ loop around the quantizer, with a loop filter setting the noise shaping. $\Sigma \Delta$ ADCs are well-studied and versatile architectures which only miss one important feature: a highly-accurate analytical model.

4 Systematic Design of $\Sigma \Delta$ ADCs 


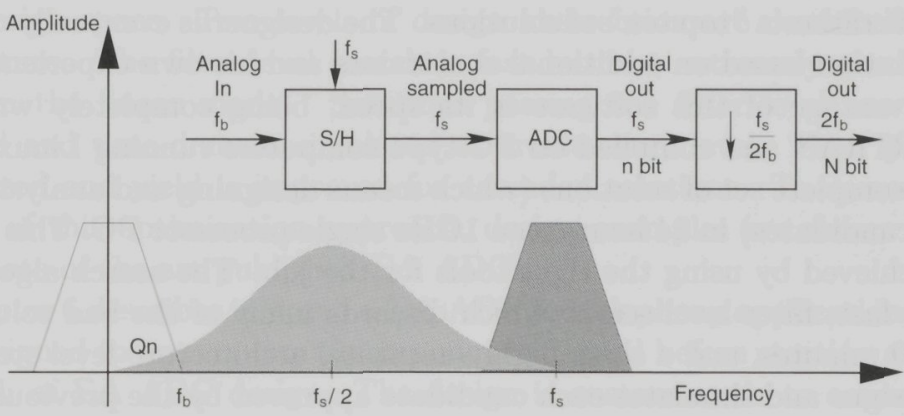

Figure 1.3: Noise shaping ADC

\subsection{Motivation}

The $\Sigma \Delta$ ADC is probably the most used ADC architecture nowadays. These ADCs fit perfectly on the same chip with digital processors, or at least better than other architectures. They are very versatile, converting from seismic signals in the milihertz range up to communication signals reaching into tens of megahertz, with resolutions of 10, 16 or 24 bits. Their complexity ranges from the simplest first-order with a single-bit quantizer to cascaded fifth-order with multi-bit quantizers in each loop. Most of the reported designs are based on industry's "trusted solutions" since no accurate analytical model is available for the general case.

A $\Sigma \Delta$ ADC cannot be just calculated at architecture-level. Its behavior, especially for large order loop filters, is governed by the presence of a signaldependent quantization noise instead of the white quantization noise assumed by the linear model. While the quantization noise power can be calculated based on linear approximations of the loop filter, there is no mathematical model to predict what is the peak signal that can be converted. The final, completely designed $\Sigma \Delta$ ADC must be simulated in time-domain (large-signal) to find $N$, the final number of bits of resolution at Nyquist rate. And if $N$ is not convenient, another design cycle must be started, sometimes after a topology change.

This thesis is an attempt to make a systematic approach on the design of $\Sigma \Delta$ ADCs, regardless of order, OSR, topology, number of cascaded loops and number of quantizer bits. The methods and models described here have been used to write a set of CAD programs which perform exhaustive search of the entire $\Sigma \Delta$ ADC design space for a given set of targets like resolution and signal bandwidth. The exhaustive search is the only practical option since no mathematical model is available for the $\Sigma \Delta \mathrm{ADC}$ behavior, and therefore no gradient methods can be used for global optimization. The search is oriented toward minimizing the power consumption of the final design and it supplies not 


\section{Introduction}

only one but rather a "top ten" of solutions. The designer is eventually choosing the best solution based on additional constraints and his own experience.

The advantage of this software is its speed, being completely written in $\mathrm{C}$ and FORTRAN and compiled on PC-type computers running Linux. It can generate a complete set of solutions (which means designing and analysing more than 2000 candidates) in 24 hours on a $1 \mathrm{GHz}$ single-processor PC. This speed is not only achieved by using the right tools for the job. The search algorithm is split into a fast, filter-level search which discards many of the bad solutions in less than 10 minutes, and a slow, highly-accurate, architecture-level step which actually designs and simulates each candidate approved by the previous step as a potential solution.

\subsection{Thesis Organization}

This thesis is divided in seven chapters describing the different aspects of the $\Sigma \Delta$ ADC design process.

Chapter 2 covers in detail the architecture-level design of $\Sigma \Delta$ ADCs having both discrete-time and continuous-time loop filters. The principle of operation of $\Sigma \Delta$ ADCs is explained based on the linear model and the limitations of this model are introduced. An example of mapping the loop filters on a given topology is given, and the equivalence between discrete-time and continuoustime loop filters is explained. A few non-ideal circuit effects are described, which are easy to analyze at architecture-level. The chapter covers both single-loop and cascaded (MASH) topologies.

Chapter 3 describes the circuit blocks typically used to implement discretetime $\Sigma \Delta$ ADC loop filters. The topology, non-ideal effects and power consumption of switched-capacitor (SC) integrators are analysed. The power model starts from the target noise and distortion performance to calculate the capacitors in the integrator and derive a compact expression for the opamp transconductance, a good estimate of the power consumption. Other blocks, like zerodelay SC amplifiers and dynamic comparators, are also described. The design of multi-bit quantizers and DACs for differential circuits is also explained.

Chapter 4 follows the same structure but targets continuous-time (CT) loop filter design. The active RC integrator is analyzed in detail as being the most linear option. The pros and cons of different DAC topologies are explained and non-ideal circuit-level effects are discussed. Again, a model of the power consumption is derived, calculating the opamp transconductance from the noise budget and distortion target. A quantizer suited for connection to CT integrators is explained. Multiple options of implementing DAC functionality, including multi-bit calibrated DACs, are detailed.

Chapter 5 covers both the numerical methods and the decision algorithms used to implement an exhaustive search of a global optimum in the entire $\Sigma \Delta$

6 Systematic Design of $\Sigma \Delta$ ADCs 
ADC design space. Two levels of design abstraction are described, filter and architecture. The filter-level search is fast but not extremely accurate, being based on the linear model. Solutions passing the filter level are completely designed and time-domain simulated at architecture level. Their performance is analyzed and yield is improved for cascaded solutions. To demonstrate the validity of this optimization approach, design examples are shown for targets set to state-of-the-art published $\Sigma \Delta$ ADCs.

Chapter 6 describes in detail a $\Sigma \Delta$ ADC for telephony applications which has been designed to accumulate the experience needed before starting a systematic approach of $\Sigma \Delta \mathrm{ADC}$ design. The design is custom-made to connect directly to an electret microphone without the need of a pre-amplifier. Single-ended to differential conversion takes place in the first integrator which also offers a high-impedance input to isolate the electret from the rest of the circuit. The power consumption is reduced by using a mixed CT/DT loop filter.

Chapter 7 describes an optimal design as generated by the CAD software. It is a broadband $\Sigma \Delta \mathrm{ADC}$ targeted toward wire communication applications of DSL type. Its power consumption is reduced by keeping the OSR low while increasing the number of quantizer bits. The loop filter is designed with a continuous-time first integrator for low power consumption, and discretetime (switched-capacitor) higher-order integrators for better loop stability with highly-aggressive noise transfer function. The DAC of the first integrator is calibrated using a novel, active calibration technique which compensates for both dynamic and static DAC cell mismatches. 
REFERENCES

\section{References}

[1] V. Bush, As We May Think, The Atlantic Monthly, Vol. 176, No. 1, July 1945.

[2] R. van de Plassche, Integrated Analog-to-Digital and Digital-to-Analog Converters, Kluwer Academic Publishers, Boston, 1994.

[3] S.R. Norsworthy, R. Schreier, G.C. Temes, Delta-Sigma Data Converters, IEEE Press, 1997. 


\section{2}

\section{Architecture-Level Analysis of Sigma-Delta ADCs}

A $\Sigma \Delta$ ADC requires a set of two transfer functions to be implemented. In the widely-used case of the low-pass ADCs, the two functions are a high-pass noise transfer function (NTF) and a low-pass signal transfer function (STF). They are implemented with an architecture containing a loop filter and a coarse quantizer (with lower resolution than the Nyquist-rate target) which limits the applicability of the analytical (linear) model for the noise-shaping ADC [1]. These limits of linear model can only be overcome by time-domain simulations of the fully-designed architecture. Architecture-level issues of $\Sigma \Delta$ ADC design are covered in this Chapter, from effects of NTF and STF design choices to effects of circuit-related non-idealities like clock jitter, which are easy to model accurately at this abstraction level [2]. Section 2.1 describes the linear model, principles and operation of a $\Sigma \Delta$ ADC. In Section 2.2 , the design of both singleloop and cascaded, discrete-time (DT) $\Sigma \Delta$ ADCs is covered. A similar analysis is carried out for the continuous-time (CT) $\Sigma \Delta$ ADCs in Section 2.3.

\subsection{Principle and Operation}

\subsubsection{Linear Model. Transfer Functions}

A generic discrete-time representation of a $\Sigma \Delta$ ADC, given in Fig. 2.1, is best used to explain the functioning of the ADC modeled as a linear system. The loop filter has two sections, a forward filter $\mathrm{G}(\mathrm{z})$ and a feedback filter $\mathrm{H}(\mathrm{z})$. The input signal $\mathrm{X}(\mathrm{z})$ is applied and compared with the signal fed back by $\mathrm{H}(\mathrm{z})$, filtered through $G(z)$ and quantized to give the digital output. The quantization introduces an error $\mathrm{E}(\mathrm{Z})$ which is modeled as input-signal-independent 


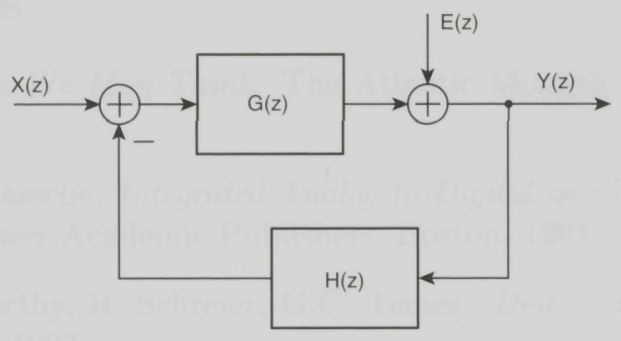

Figure 2.1: Noise shaper

and directly added to the output, in the quantizer (represented as a summation point).

The two $\Sigma \Delta$ transfer functions are defined on the system above: the signal transfer function to characterize the transfer from $\mathrm{X}(\mathrm{z})$ to $\mathrm{Y}(\mathrm{z})$ and the noise transfer function for the partial contribution of $\mathrm{E}(\mathrm{z})$ in $\mathrm{Y}(\mathrm{z})$

$$
Y(z)=\operatorname{STF}(z) X(z)+N T F(z) E(z)
$$

These functions can be independently defined because a linear model for the quantizer is assumed, thus making the whole system a linear one where superposition rules apply. The definitions of the two transfer functions are based on the two sections of the loop filter:

$$
\begin{aligned}
& \operatorname{STF}(z)=\frac{Y(z)}{X(z)}=\frac{G(z)}{1+G(z) H(z)} \\
& \operatorname{NTF}(z)=\frac{Y(z)}{E(z)}=\frac{1}{1+G(z) H(z)}
\end{aligned}
$$

The STF can be approximated with 1 (one) at frequencies where $\mathrm{G}(\mathrm{z})$ is large and $\mathrm{H}(\mathrm{z})$ is unity, while, at the same frequencies, NTF can be approximated with 0 (zero) [1]. Note that, if the filters are implemented using integrators, the frequency band where $G(z)$ is large is around DC (see Fig. 2.2). If resonators are used instead of integrators, the central frequency for the region of interest is shifted around the resonance frequency of the resonators. The latter approach allows for bandpass converters to be designed.

The feedback filter $\mathrm{H}(\mathrm{z})$ is usually not implemented separately, but as distributed feedbacks into the G(z) [3]. Simpler systems can be used but they only allow full control of one of the two transfer functions. Because the NTF limits the quantization noise power which gives the conversion resolution, this transfer function is designed for the target performance. STF results as the inverse of NTF which means 0dB gain inside the signal band and no independent control whatsoever outside that band, as it will be explained in sub-Section 2.2.2. 


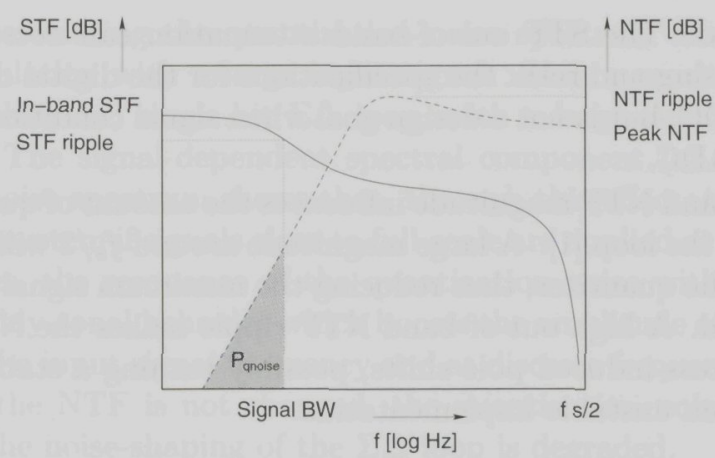

Figure 2.2: NTF and STF amplitude characteristics for a low pass $\Sigma \Delta A D C$

Typical shapes for NTF and STF are shown in Figure 2.2. The full line represents the STF while the dashed line is a typical NTF for a low-pass $\Sigma \Delta$ converter. The important parameters of the two curves are shown, for STF the $\mathrm{DC}$ value and in-band ripple and for NTF the $f_{s} / 2$ gain and out-of-band ripple. It is worth noting that the STF gain is dropping out of the band, rejecting high frequency signals. When STF is not well controlled it can also show an overshoot just above signal band limit, which is tightening the rejection specification for the digital filter following the converter [1]. A steeper (higher-order) digital filter may be needed to reduce the amplitude of any input spectral components occurring at those frequencies.

The steepness of the NTF curve inside the signal band is given by the order of the numerator of the product $\mathrm{G}(\mathrm{z}) \mathrm{H}(\mathrm{z})$ (see Fig. 2.1) multiplied by $20 \mathrm{~dB} / \mathrm{dec}$. The poles of this product give the zeros of NTF while the zeros of the $G(z) H(z)$ product affect the poles of NTF. Using resonators inside the $\mathrm{G}(\mathrm{z})$, the NTF zeros can be moved away from DC and spread inside the signal bandwidth in a manner which minimizes total quantization noise power. This noise power, scaled by the quantization noise spectral density, is represented on the graph in Fig. 2.2 as the shaded triangle marked $P_{\text {qnoise }}$ which gives the hard (theoretical) limit for the conversion resolution in the given band, for a given NTF and quantization noise total power. In an oversampled ADC, the input signal bandwidth Signal $B W$ is lower than half the sampling frequency, and a design parameter, the oversampling ratio OSR, shows how many times the Nyquist theorem is overapplied

$$
O S R=\frac{f_{s} / 2}{\text { Signal } B W}
$$

A large STF ripple is not convenient for most applications, which do not tolerate frequency-dependent in-band gain. For certain applications the STF phase is also important, as is the case with audio applications where a linear 
phase is required. The STF out-of-band attenuation can decrease the risk of input signal aliasing and relax the specifications for the digital decimating filter following the ADC, but is not a design goal when signal conditioning is available in front of the ADC.

The out-of-band NTF magnitude influences the amount of quantization noise re-circulated on the loop [1]. A large magnitude around $f_{s} / 2$ will put more noise at the input of the quantizer, thus reducing the maximum signal level which can still be processed. A high out-of-band NTF ripple makes the NTF shape more sensitive to process-induced pole shifts, possibly turning a stable design into a less-stable or even unstable implementation.

\subsubsection{Non-Linear Loop Transfer}

The linear model only yields accurate results if the two signals entering the system and used to define the transfer functions, $X(z)$ and $E(z)$ in Fig. 2.1, are mutually independent. This is true for small input signals, when the quantizer input is dominated by re-circulated quantization noise. At high input signals however, the signal at the input of the quantizer also contains some of the input signal spectrum. Since the quantizer output can only take fixed values, the noise added in the quantizer is no longer signal-independent. This can

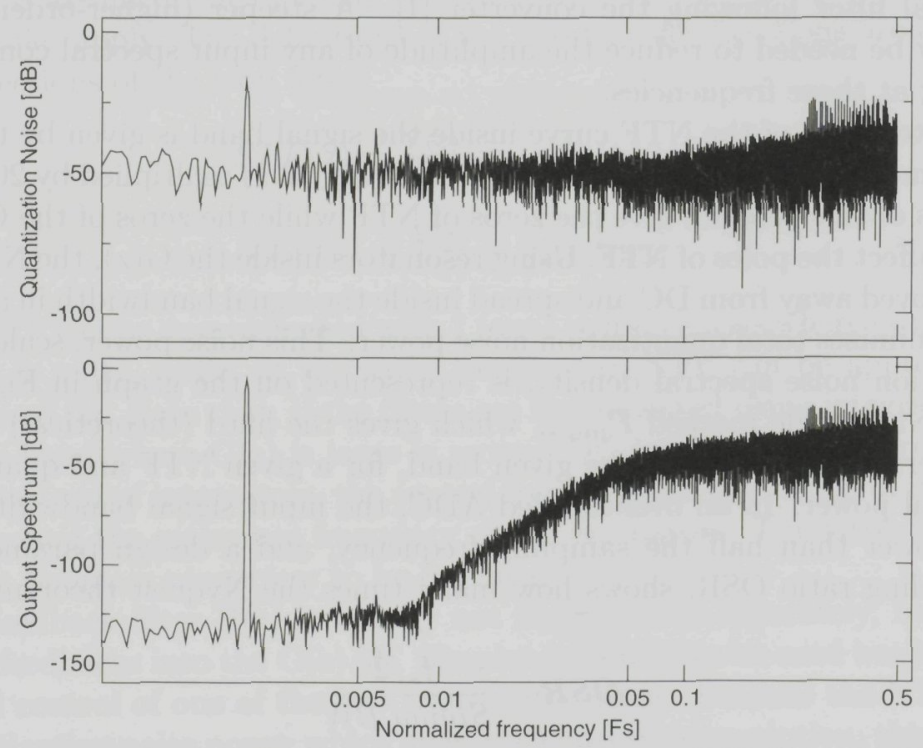

Figure 2.3: Output (bottom) and quantization noise (top) spectra for a fourthorder, single-bit $\Sigma \Delta A D C$ 
be found by calculating the quantization noise spectrum from a time-domain $\Sigma \Delta$ ADC simulation with a large input signal. Such a result is shown in Fig. 2.3 for a fourth-order, single-bit $\Sigma \Delta$ loop, with a large-amplitude, single-tone input signal. The signal-dependent spectral component (at $0.0039 \mathrm{Fs}$ ) in the quantization noise spectrum shows that, through this effect, the $\Sigma \Delta$ loop is no longer a linear system if signals close to full-scale are applied at the input. Under such conditions, the resonance of the quantization noise with the input signal produces a highly-tonal behavior which boosts the amplitude of the quantization noise around the input signal frequency and at discrete frequencies derived from it. Although the NTF is not changed, the quantization noise filtered by it is changed and the noise-shaping of the $\Sigma \Delta$ loop is degraded.

For input signal amplitudes above a certain level, called the overloading level $(\mathrm{OVL})$ and expressed in $\mathrm{dB}$ to reference level $(\mathrm{dBR})$, the loop does not work as a noise shaper anymore and more noise is present inside the band of interest. As it can be seen in Fig. 2.4, the output and quantization noise spectra for the same design used to generate Fig. 2.3 are completely modified due to overload. Fig. 2.3 has been generated with an input signal 1dBR lower than OVL, Fig. 2.4 for 1dBR higher than OVL. The input tone is boosted in the output spectrum from the expected $-3.5 \mathrm{dBR}$ to almost $+1 \mathrm{dBR}$ by the resonance effect. The lowfrequency quantization noise power is dramatically increased and re-distributed

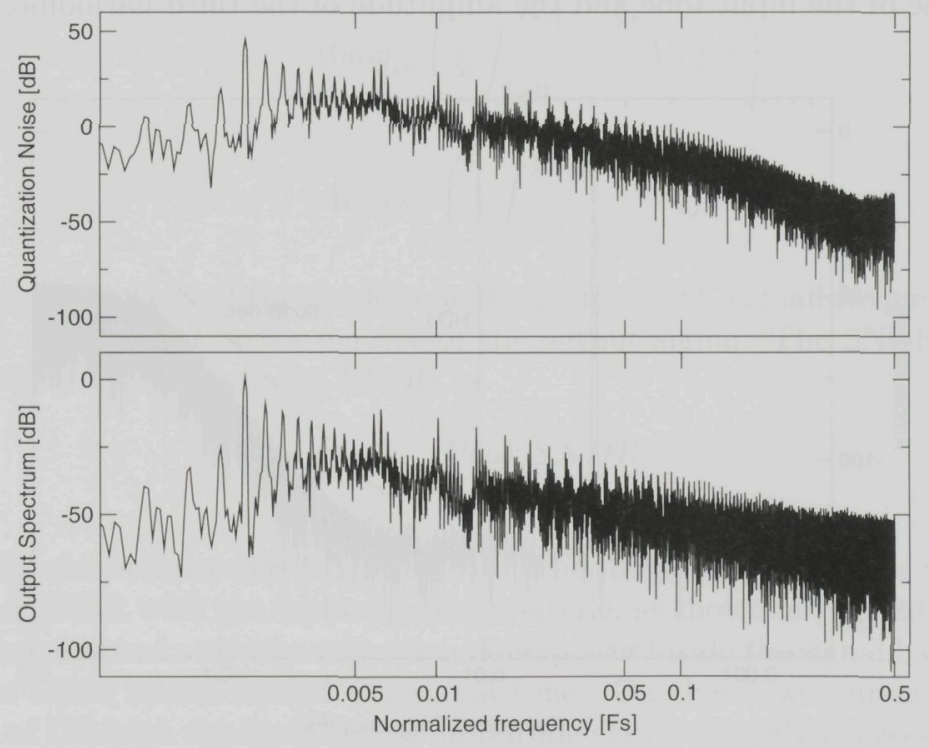

Figure 2.4: Output (bottom) and quantization noise (top) spectra for a fourthorder, single-bit $\Sigma \Delta A D C$ in overloading condition 
from the flat, white-noise-like density in Fig. 2.3, to a spectral density showing strong modulation around the input signal tone.

The OVL value varies with $\Sigma \Delta$ loop order, loop topology, quantizer resolution and NTF aggressiveness. In systematic $\Sigma \Delta$ ADC design, these parameters along with OSR define the filter-level design space (as it will further be explained in Chapter 5) where, under the limits imposed by the overloading effect, the linear model can be used to estimate the performance of a $\Sigma \Delta$ ADC design.

\subsubsection{Performance Metrics}

During the design and characterization of a $\Sigma \Delta$ ADC, a number of performance metrics are collected from output spectra of the ADC converting different types of input signals. Such a spectrum is shown in Fig. 2.5, generated by a fast Fourier transform (FFT) of the digital output of a fourth-order $\Sigma \Delta$ ADC with a 5-bit quantizer. The input signal is set close to the OVL, at $-2 \mathrm{dBR}$. The entire spectrum is scaled to bring the reference level to $0 \mathrm{dBR}$, so all the spectral components are expressed in $\mathrm{dBR}$. On such a graph it can be measured if the noise shaping has the correct order (here the quantization noise raises with $80 \mathrm{~dB} /$ decade outside the signal bandwidth), showing the loop is not overloaded. The resolution is also measured on this spectrum, as it will be explained in the next paragraph, as well as the distortion, here shown as the ratio between the amplitude of the input tone and the amplitude of the third harmonic, HD3.

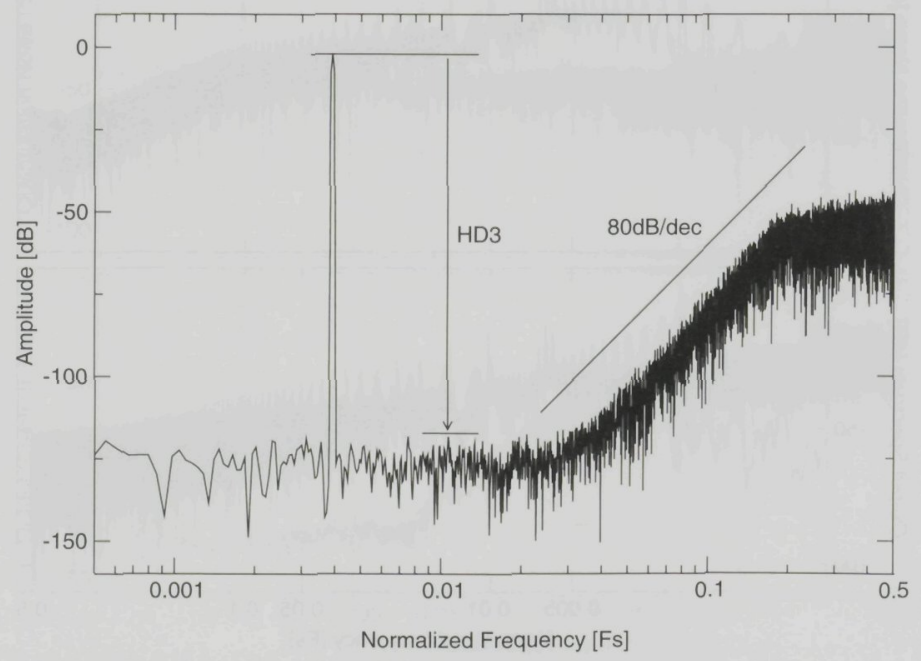

Figure 2.5: FFT spectrum for the output digital stream of a fourth-order, 5-bit $\Sigma \Delta A D C$ with peak input signal amplitude at $-2 d B R$ 
Similar with other ADCs, the resolution of a $\Sigma \Delta$ ADC is the measure for the smallest analog signal which can be converted. Only in the case of $\Sigma \Delta$ converters, due to the quantization noise particular shape, the definition of the resolution does not start from the noise floor level, but rather from the total in-band noise power which gives the dynamic range (DR)

$$
D R=-10 \log _{10}\left(\frac{1}{2} \int_{f=0}^{f=f_{b}} Y(f)^{2}\right)[d B]
$$

with $f_{b}$ being the input signal bandwidth (Signal $B W$ in Fig. 2.2) and $Y(f)$ being the quantization noise spectral power density, measured as the spectrum of the digital stream output with no input signal.

Because of the overloading effect, DR cannot be used to define the effective number of bits (ENOB) since the full input scale is not at 0dBR. In the same time, DR does not explicitly include non-linearity information. To determine ENOB, the ratio between the input signal power and the power of all the other in-band spectral components is calculated as the signal-to-(noise+distortion) ratio SNDR

$$
\begin{aligned}
S N D R= & 10 \log _{10}\left(\frac{1}{2} \int_{f=f_{i n}-\delta f}^{f=f_{i n}+\delta f} Y(f)^{2}\right) \\
& -10 \log _{10}\left(\frac{1}{2} \int_{f=0}^{f=f_{i n}-\delta f} Y(f)^{2}\right) \\
& -10 \log _{10}\left(\frac{1}{2} \int_{f=f_{i n}+\delta f}^{f=f_{b}} Y(f)^{2}\right)
\end{aligned}
$$

with $f_{i n}$ the frequency of the single-tone input signal, $\delta f$ a small frequency shift and $Y(f)$ the spectral power density of the output signal. The SNDR is used to approximately calculate the ENOB as

$$
E N O B \approx \frac{P e a k S N D R}{6}
$$

To fully characterize the $\Sigma \Delta \mathrm{ADC}$, two other curves are used: the variation of DR and SNDR with the input signal amplitude, as shown in Fig. 2.6. In this case DR is measured as the quantization noise power inside the signal bandwidth except for small bands around the signal tone. On these two curves, a large variation of DR with the input signal amplitude shows a highly-aggressive NTF or circuit-induced non-ideal effects, the differential non-linearity (DNL) can be measured on the SNDR curve as local changes of the slope and integral nonlinearity (INL) as a variation of the average slope from the desired conversion 


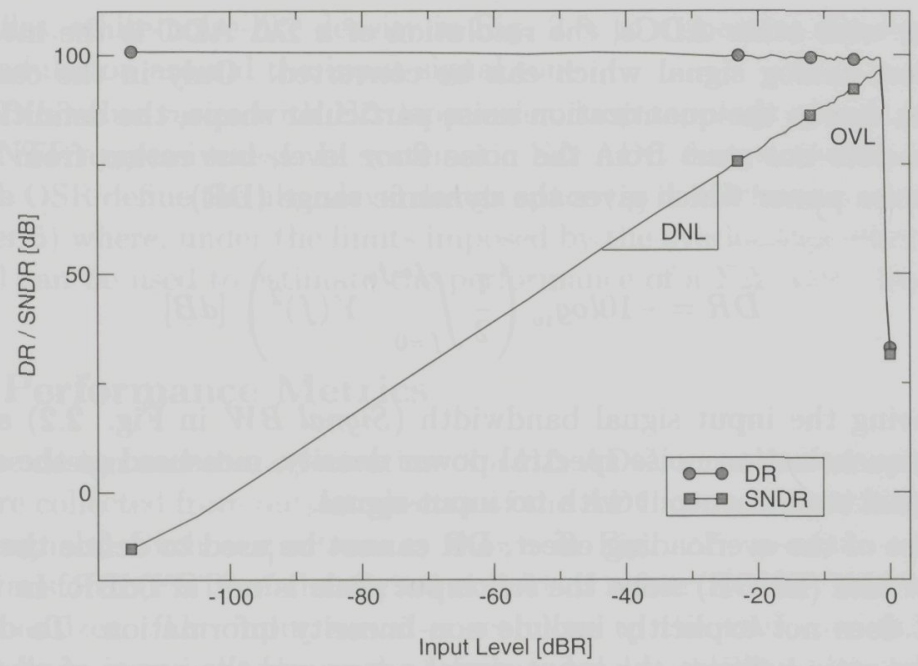

Figure 2.6: SNDR and DR variation with the input signal amplitude

gain. These measurements are performed from the OdB SNDR point (here at $-100 \mathrm{dBR}$ input) up to the OVL as defined by the peak value of the SNDR.

The OVL can also be looked upon as a performance metric, although its effect is also accounted for in ENOB by considering the OVL to be the full-scale input signal, used to calculate the Peak SNDR. In the systematic design of $\Sigma \Delta$ ADCs, the performance metrics are set as targets. Not meeting one of them disqualifies a solution.

\subsection{Sigma-Delta ADCs with Discrete-Time Loop Filters}

\subsubsection{Design of Loop Transfer Functions}

The NTF is the filter defining the resolution and other properties of a $\Sigma \Delta$ ADC, hence the design process always starts by defining this transfer function. Even if different filter families (Butterworth, Chebyshev or elliptic) can be used since the filter family has never been found to significantly affect the performance of a $\Sigma \Delta$ ADC [4], an NTF filter has to be causal and scaled to yield an impulseresponse starting with a unitary output. The causality is required for a filter mapped to physical systems and implies that there cannot be more zeros than poles in the $\operatorname{NTF}\left(z^{-1}\right)$. The requirement of initial unitary impulse-response stems from the architecture of a $\Sigma \Delta \mathrm{ADC}$, as shown in the previous section, 
Fig. 2.1. There is no direct (zero-delay) feedback from the quantizer output to the quantizer input. All the feedback is supplied through the loop filter $G\left(z^{-1}\right) H\left(z^{-1}\right)$ which contains at least one delay in its forward path. Hence, according to the expression of $\operatorname{NTF}\left(z^{-1}\right)$ derived in Eq. 2.2, the ratio of the zero-order terms in both the numerator and denominator of $N T F\left(z^{-1}\right)$ is always one. This ratio sets the initial impulse response of the filter.

The required scaling of the NTF filter introduces a relationship between its cutoff $(-3 \mathrm{~dB})$ frequency and its magnitude at half the sampling frequency $f_{s} / 2$. An increased cutoff frequency induces a larger magnitude at $f_{s} / 2$, as shown in Fig. 2.7 for the NTF of a fourth-order $\Sigma \Delta$ ADC with Chebyshev poles. The full line has the lowest cutoff frequency and the smallest magnitude in the same time. It is the least aggressive NTF in the set shown. The legend in the graph also shows simulated DR for the three curves designed into singlebit $\Sigma \Delta \mathrm{ADC}$ architectures, and the least aggressive curve offers the lowest DR $(66 \mathrm{~dB})$ from the three. This is caused by a larger in-band (DC to Signal BW limit) quantization noise power compared with the more aggressive members of the set. Under the assumption of flat quantization noise spectrum, the ratio between different $\mathrm{DR}$ values is given by the ratio of triangular areas defined by the in-band NTF magnitude and the straight line marked Signal BW setting the limit of signal bandwidth.

From the point of view of attainable DR it is obviously more convenient to use aggressive NTF filters. There is however a price to pay for the NTF

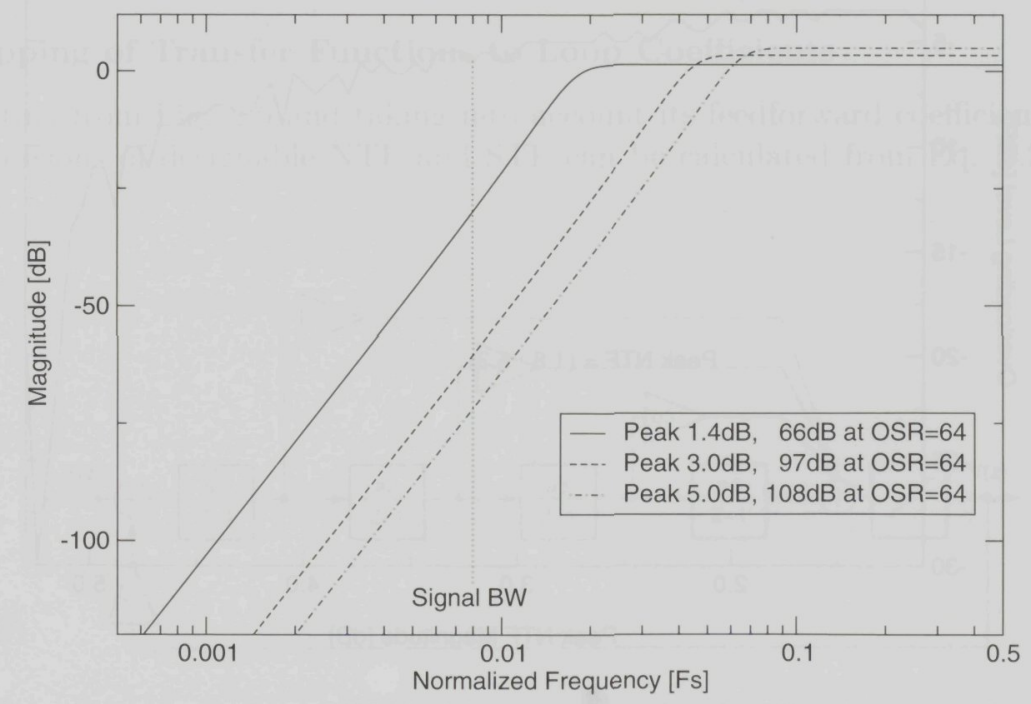

Figure 2.7: Choice of NTF aggressiveness for a fourth order $\Sigma \Delta A D C$ 
aggressiveness, and this is a reduced overloading level (OVL). As explained in the previous Section, a lower-than-0dBR OVL is produced by the modulation of the quantization noise with the input signal spectral components. A more aggressive NTF concentrates more quantization noise power around $f_{s} / 2$ for small input signal levels. Hence, at large input signal levels, larger spectral components will be found in the quantization noise spectrum around the input signal frequencies, producing faster overloading due to increased non-linear $\Sigma \Delta$ loop transfer. Fig. 2.8 shows the variation of OVL as a function of the peak NTF magnitude (NTF magnitude at $f_{s} / 2$ for flat out-of-band NTF) for a fourthorder single-bit $\Sigma \Delta$ ADC. The peak NTF magnitude is used to measure its aggressiveness rather than the cutoff frequency in order to highlight the effect explained above. The number of bits in the quantizer has to be taken into account since it changes the power of the quantization noise and the tonal behavior of the quantization noise at large input signals. This curve, connected with the DR values shown in Fig. 2.7, shows the trade-off between the attainable DR and peak SNDR (if limited only by OVL).

The graphs in Figs. 2.7 and 2.8 also contain the limits of the usable NTF aggressiveness for a fourth-order, single-bit $\Sigma \Delta$ ADC. It is impossible to design a stable $\Sigma \Delta$ ADC with this architecture if the peak NTF magnitude falls outside the shown limits [1].

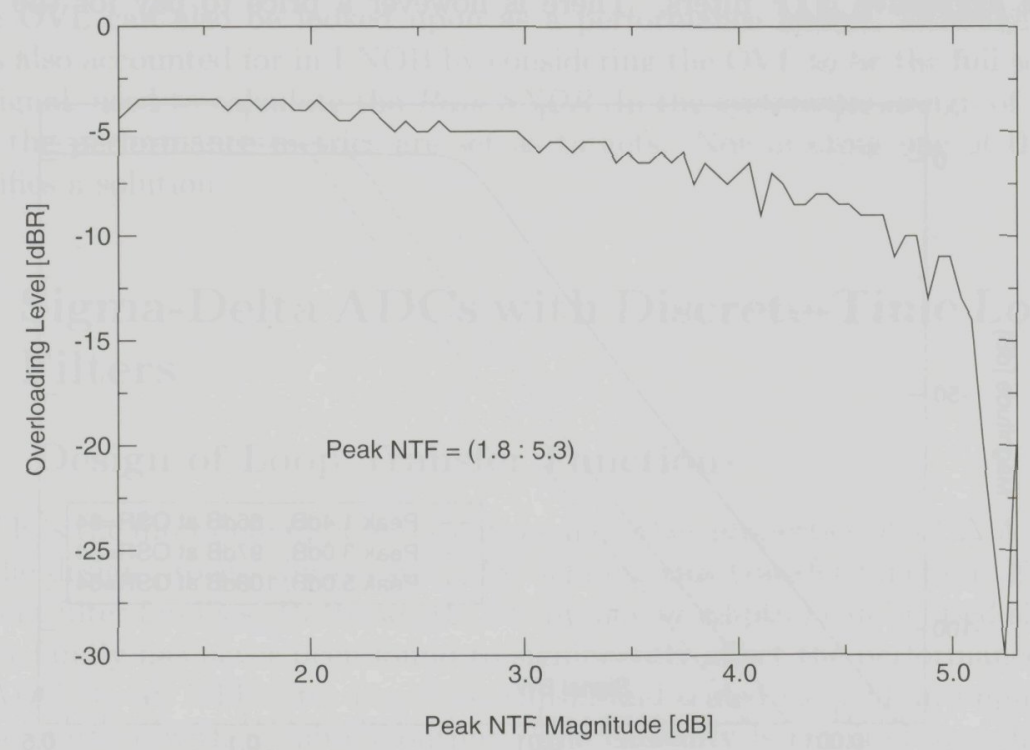

Figure 2.8: Overloading level variation with NTF aggressiveness for a fourth order, single-bit $\Sigma \Delta A D C$ 


\subsubsection{Single Loop Topologies}

Different topological variants can be used to design "stable" (or rather "realizable") $\Sigma \Delta$ ADCs. No topology design can stop the non-linear behavior of the $\Sigma \Delta$ loop to become apparent at high input signals, therefore the overloading level is less than 0dBR for all implementations. However, some topologies behave better than others at large input signals in terms of OVL value, increasing it by one or two dBR. OVL is much tighter connected with the NTF aggressiveness than with the $\Sigma \Delta$ topology used. Why "realizable" is a better term than "stable" when it comes to characterizing a topology becomes apparent by taking a look at Fig. 2.9 which is a typical fourth-order "realizable" topology, sometimes praised to be the most "stable" one due to its feedforward coefficients $b_{1} \cdots b_{3}[5]$. Without the feedforward coefficients, the only NTF that can be mapped on this topology would be

$$
\operatorname{NTF}(z)=\frac{(z-1)^{4}}{(z-1)^{4}+f_{1} a_{1} a_{2} a_{3} a_{4}}
$$

The $\operatorname{NTF}(z)$ above offers very little freedom in designing its poles and is practically impossible to map on a convenient filter shape like the ones in Fig. 2.7. The fact that no usable NTF can be mapped on such a structure is a much stronger constraint in using it than instability. How the presence of the multiple feedforward or feedback coefficients adds enough freedom degrees to allow mapping of any NTF filter on this topology is explained in the following paragraphs.

\section{Mapping of Transfer Functions to Loop Coefficients}

Starting from Fig. 2.9 and taking into account its feedforward coefficients, the expressions of designable NTF and STF can be calculated from Eq. 2.2 after

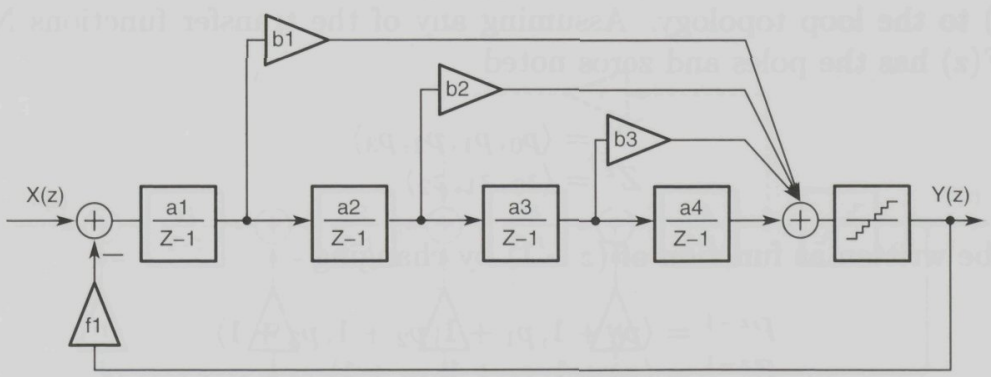

Figure 2.9: Distributed feedforward topology 
expressing $\mathrm{G}(\mathrm{z})$ and $\mathrm{G}(\mathrm{z}) \mathrm{H}(\mathrm{z})$ as sums of products of loop coefficients $a_{1} \cdots a_{4}$ and $b_{1} \cdots b_{3}$. The filter $\mathrm{G}(\mathrm{z})$ is the partial contribution of the input signal $\mathrm{X}(\mathrm{z})$ to the quantizer input

$$
G(z)=\frac{b_{1} a_{1}}{z-1}+\frac{b_{2} a_{1} a_{2}}{(z-1)^{2}}+\frac{b_{3} a_{1} a_{2} a_{3}}{(z-1)^{3}}+\frac{a_{1} a_{2} a_{3} a_{4}}{(z-1)^{4}}
$$

The other filter of interest, $\mathrm{G}(\mathrm{z}) \mathrm{H}(\mathrm{z})$, is the effect of the output signal $\mathrm{Y}(\mathrm{z})$ to the quantizer input through the feedback coefficient $f_{1}$

$$
G(z) H(z)=-f_{1} G(z)
$$

By replacing the two expressions above in Eq. 2.2, the transfer functions are easily written as $\operatorname{NTF}(z-1)$ and $\operatorname{STF}(z-1)$

$$
\begin{aligned}
& \operatorname{NTF}(z-1)=\frac{(z-1)^{4}}{\sum_{j=4}^{0} n_{j}(z-1)^{j}} \\
& \operatorname{STF}(z-1)=\frac{\sum_{i=3}^{0} m_{i}(z-1)^{i}}{\sum_{j=4}^{0} n_{j}(z-1)^{j}}
\end{aligned}
$$

with the polynomial coefficients calculated from the topology coefficients

$$
\begin{array}{ll}
n_{4}=1.0 & \\
n_{3}=f_{1} b_{1} a_{1} & m_{3}=b_{1} a_{1} \\
n_{2}=f_{1} b_{2} a_{1} a_{2} & m_{2}=b_{2} a_{1} a_{2} \\
n_{1}=f_{1} b_{3} a_{1} a_{2} a_{3} & m_{1}=b_{3} a_{1} a_{2} a_{3} \\
n_{0}=f_{1} a_{1} a_{2} a_{3} a_{4} & m_{0}=a_{1} a_{2} a_{3} a_{4}
\end{array}
$$

The NTF and STF are only represented as functions of $(z-1)$ instead of $z$ because they are easier to extract from the topology in this form, with the integrator's un-scaled transfer function being expressed as $1 /(z-1)$. The $(z-1)$ form does not affect in any way the mapping of linear-model filters $\operatorname{NTF}(z)$ and $\operatorname{STF}(z)$ to the loop topology. Assuming any of the transfer functions $\operatorname{NTF}(z)$ or $\operatorname{STF}(z)$ has the poles and zeros noted

$$
\begin{aligned}
& P^{z}=\left\langle p_{0}, p_{1}, p_{2}, p_{3}\right\rangle \\
& Z^{z}=\left\langle z_{0}, z_{1}, z_{2}\right\rangle
\end{aligned}
$$

it can be written as function of $(z-1)$ by changing

$$
\begin{aligned}
& P^{z-1}=\left\langle p_{0}+1, p_{1}+1, p_{2}+1, p_{3}+1\right\rangle \\
& Z^{z-1}=\left\langle z_{0}+1, z_{1}+1, z_{2}+1\right\rangle
\end{aligned}
$$

With the filter functions written $\operatorname{NTF}(z-1)$ and $\operatorname{STF}(z-1)$, a target set of poles gives the $n_{j}, j=0 \cdots 3$ polynomial coefficients (Eq. 2.10), which yields 
an over-determined system of four equations (Eq. 2.11). A set of STF zeros cannot be implemented from lack of freedom degrees. The feedback coefficient $f_{1}$ results from the ratio of $n_{0}$ and $m_{0}$ and sets the DC conversion gain. With $f_{1}$ calculated, the equation system built from the mapping of the $n_{j}, j=0 \cdots 3$ polynomial coefficients has four equations and seven variables, hence three of them are freedom degrees to be chosen by the designer. It is convenient to make the first three integrator coefficients $\left(a_{1}, a_{2}\right.$ and $\left.a_{3}\right)$ the freedom degrees so the designer can control these integrators' outputs, as will be explained in a latter paragraph. Once these freedom degrees set, the rest of the loop coefficients result from the values of $n_{j}$. Any set of poles can therefore be implemented using this topology since enough freedom degrees are available. It is worth noting that three feedforward coefficients are the minimal number needed to control the pole structure due to the specific form of the $n_{j}$ equation system.

A complementary topology replaces the three feedforwards with three feedback coefficients, as shown in Fig. 2.10. A system of equations can be written for this topology and used to demonstrate that the three feedback coefficients $f_{2}, f_{3}$ and $f_{4}$ are sufficient to offer control over the pole structure of the NTF and STF, hence this topology can also be used for $\Sigma \Delta$ ADC designs. Because only three feedback coefficients can be added it still lacks complete control over the STF zeros which makes the STF to be almost completely determined by the choice of NTF poles. To gain control over STF zeros as well, there is obviously need to use a more complex topology by connecting both distributed feedbacks and feedforwards. Such high-complexity topologies will be used in the design examples presented in Chapters 6 and 7 to control the STF in-band magnitude flatness and its roll-off at higher frequencies.

Note that although all four NTF zeros are shown in Eq. 2.10 as being placed at DC $(\mathrm{z}=1)$ it is possible, with minor architecture changes, to spread the zeros inside the signal bandwidth and further reduce the NTF's in-band mean value, thus reducing the total in-band quantization noise power. The use of a resonator (shown dashed in Fig. 2.10, loop coefficient $r$ ) introduces a zero in the $\operatorname{NTF}(z)$

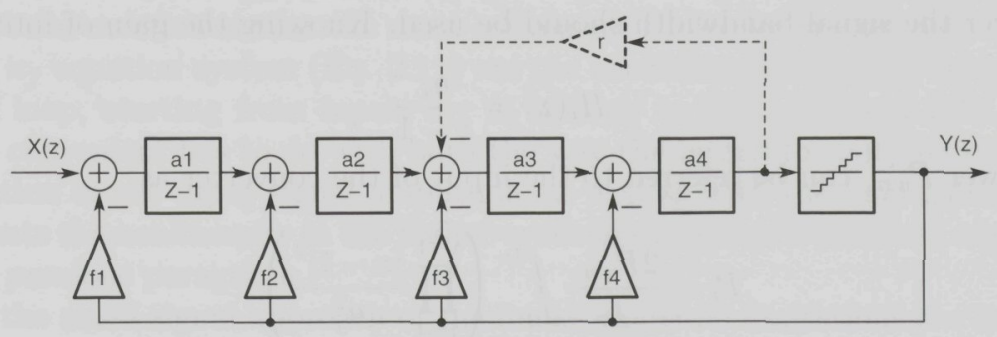

Figure 2.10: Distributed feedback topology 


\section{Architecture-Level Analysis of Sigma-Delta ADCs}

at

$$
z_{\text {in-band }}=\sqrt{1+r a_{3} a_{4}}
$$

If the resonator coefficient $r$ is considered during the initial mapping of the $\operatorname{NTF}(z)$ to the designed topology, the polynomial calculations become much more complex. Instead, taking advantage of the usually small value of $r$ caused by the small value of $z_{i n \text {-band }}$ (especially at high OSR), the resonator can be completely ignored in the first mapping phase and only calculated and added after the integrator coefficients have been determined. At low OSR the effects of the resonators over NTF poles must be studied more careful. Multiple resonators can be added to reduce the NTF in-band magnitude. Depending on the order of the loop, optimal sets of NTF zeros expressed as fractions of the input signal bandwidth have been calculated or obtained by simulations and are available in published literature [1].

\section{Noise and Distortion Contribution of Individual Integrators}

Conversion performance limits for a low-pass $\Sigma \Delta$ converter are set by the noise and distortion, which are contributed by all integrators in the $\Sigma \Delta$ loop. Noise is generated in each integrator by resistors and active devices (MOS transistors). Distortion is caused by large-signal non-linear behavior of active devices. The weights associated with the noise and distortion contribution of each particular integrator in a $\Sigma \Delta$ loop depend on the loop gain from the input to the respective integrator.

For the following calculations only the white noise will be considered, generated in a bandwidth larger than half the sampling frequency, $f_{s} / 2$. Noting the total noise power at the input of each integrator $P_{w n_{i}}, i=1 \cdots 4$ (for a fourth order loop as shown in Figs. 2.9 or 2.10), the noise contribution of the first integrator to the input-referred total noise power is

$$
P_{1}=\frac{P_{w n_{1}}}{O S R}
$$

For the following integrators an integral of the total gain of previous integrators over the signal bandwidth should be used. Knowing the gain of integrator $i$ is

$$
H_{i}(z)=\frac{a_{i}}{z-1}
$$

the power $P_{w n_{i}}$ can be referred to the input of the converter as

$$
P_{i}=\frac{2 P_{w n_{i}}}{f_{s}} \int_{0}^{f_{b}}\left(\prod_{j=1}^{i-1} \frac{|z-1|^{2}}{a_{j}^{2}}\right)
$$

The product of $a_{j}$ coefficients is constant over frequency, so only the absolute value of $(z-1)$ should be integrated over signal bandwidth, form DC to the 
upper limit $f_{b}$,

$$
P_{i}=\frac{2 P_{w n_{i}}}{f_{s}}\left(\prod_{j=1}^{i-1} \frac{1}{a_{j}^{2}}\right) \int_{0}^{f_{b}}|z-1|^{2(i-1)}
$$

The term to be integrated can now be written as

$$
\begin{aligned}
|z-1|^{2(i-1)} & =\left(\left(e^{J 2 \pi f T_{s}}-1\right)\left(e^{-J 2 \pi f T_{s}}-1\right)\right)^{2(i-1)} \\
& =\left(2 \sin \left(\pi f T_{s}\right)\right)^{2(i-1)} \\
& \approx\left(2 \pi f T_{s}\right)^{2(i-1)}
\end{aligned}
$$

if the frequency of interest $f$ is much smaller than $f_{s}=1 / T_{s}$. Equation 2.18 becomes under these conditions

$$
P_{i}=P_{w n_{i}}\left(\prod_{j=1}^{i-1} \frac{1}{a_{j}^{2}}\right) \frac{\pi^{2(i-1)}}{(2 i-1) O S R^{2 i-1}}
$$

The distortion introduced by integrator $i$, noted $D_{n_{i}}$ for similarity with the noise contributions, refers back to the input of the loop by the worst-case integrator gain, which is at the end of the input signal bandwidth,

$$
D_{i}=D_{n_{i}}\left(\prod_{j=1}^{i-1} \frac{1}{a_{j}^{2}}\right)\left(\frac{1}{|(z-1)|}\right)^{(i-1)} \text { at } f=f_{b}
$$

which again, assuming a large OSR, gets a simplified form of

$$
D_{i}=D_{n_{i}}\left(\prod_{j=1}^{i-1} \frac{1}{a_{j}^{2}}\right)\left(\frac{1}{\pi O S R}\right)^{(i-1)}
$$

\section{Sizing of Free Integrator Coefficients}

It has been mentioned that the most convenient choice for the freedom degrees in the $n_{j}$ equation system (Eq. 2.11) are the coefficients of the first integrators in the loop, starting from input, $a_{1}, a_{2}$ and $a_{3}$ in Figs. 2.9 and 2.10. Why this is convenient has to do with both the way the integrator outputs evolve as a function of the input signal amplitude, as well as with the loop gains which attenuate the nonlinearity in the higher-order integrator as it has been explained in the previous paragraph.

In the small-signal approach of the linear model no limitations are imposed on the integrator outputs. In real circuits however, the voltage at any node in the topology cannot be larger than the supply voltage (as a hard limit) and is usually conveniently bound by design to values within a range where the 
active circuits operate with required performance (soft limit). For example, the amplifier inside an integrator has a decreased gain if its output gets close to a supply rail and this can in turn affect the similarity of the real integrator with the ideal one used in the linear model.

The integrator outputs (or states) are input-signal dependent [3]. A larger input signal produces larger integrator outputs, up to a point where one or more outputs reach the soft or hard limits and the conversion performance is reduced. If a soft limit is reached first, distortion is introduced in the conversion and SNDR drops. If the outputs further increase and a hard limit is also reached, the shape of the NTF is also affected and the DR decreases. Therefore, the outputs of all integrators must be kept within convenient ranges for input signal amplitudes up to the maximally convertible value set by OVL. The variation of integrator outputs with the input signal level resulted from behavioral simulations of a fourth-order, single-bit loop is shown in Fig. 2.11. The NTF implemented by this loop produces an OVL of $-6 \mathrm{dBR}$ and the freely-chosen first three integrator coefficients have been designed to keep the output of the first three integrators below the hard limit set by Vclip. The first integrator has actually been down-sized to allow the use of an opamp in the first integrator with a much lower soft limit, in the range of $0.5 \mathrm{Vclip}$, while still keeping the distortion levels below the design target. The second, third and fourth integrators gradually clip to the hard limit as the input signal level increases above OVL. This is convenient as a stabilization method for faster recovery from overload-

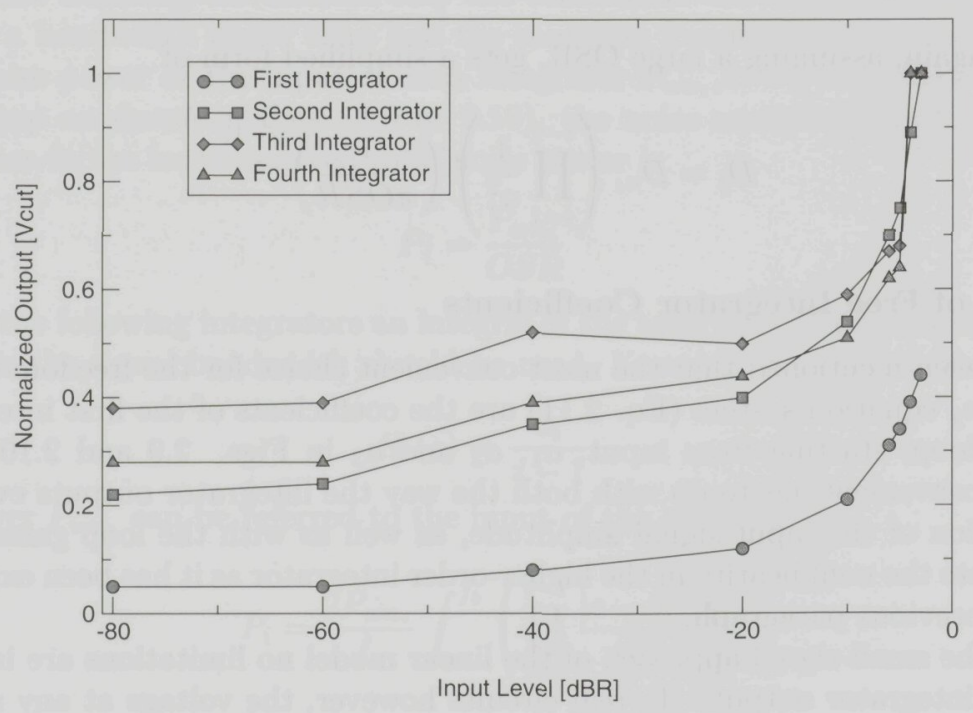

Figure 2.11: Integrator output vs. input signal amplitude 
ing condition, since in hard overloading only one integrator eventually remains active. The freely-chosen integrator coefficients (as explained with Eq. 2.11) must be designed so clipping occurs in the corresponding integrators for input levels around OVL. If a highly-aggressive NTF is used, integrator clipping can also be employed as a power-on stabilization technique [1] [3].

\section{Performance Sensitivity to Coefficient Mismatch}

Starting from the linear model, a set of loop coefficients are calculated to match the target NTF perfectly. In the real circuit however, these coefficients are implemented as ratios of currents or capacitors which only match with a certain accuracy, resulting in random coefficient-to-coefficient mismatch which shifts the position of the NTF poles, altering its shape. As a random process, the mismatch is characterized by a normal distribution with mean value zero and a standard deviation $\sigma$. The value of $\sigma$ depends on the IC process parameters and also on the size of the coefficients. Knowing that more than $99 \%$ of the mismatch occurences in a statistically-significant set of ICs (in other words large and spread on different wafers and batches) will have an absolute value smaller than $3 \sigma$, the standard deviation is used to calculate a production yield for a designed $\Sigma \Delta$ ADC. Because the coefficient mismatch introduces changes of NTF shape, more noise is expected to be present inside the signal bandwidth, therefore the DR is expected to be reduced.

One very convenient technique of improving the mismatch-limited yield for single-loop $\Sigma \Delta$ ADCs is to design the NTF to offer a margin between the target DR and the quantization-noise limited DR. In other words, the NTF is designed more aggressive than ideally needed for a given OSR, and the circuit-generated white noise power is allowed to dominate the in-band quantization noise power. By so designing the $\Sigma \Delta \mathrm{ADC}$, the DR is actually determined in a large measure by the white noise of the circuit whose power is constant for a given temperature.

The results for simulated yield of a fourth-order, single-bit $\Sigma \Delta \mathrm{ADC}$ with target white-noise limited DR (DRwn) of $90 \mathrm{~dB}, 96 \mathrm{~dB}$ and $102 \mathrm{~dB}$ at OSR $=64$ are shown in Fig. 2.12. The coefficients are assumed to show a process-wide mismatch $3 \sigma$ value of $1 \%$ (fair value for capacitor/capacitor mismatch). The margin between DRwn and the quantization noise DR (DRqn) is varied between $3 \mathrm{~dB}(66 \%$ of the noise budget taken by the white noise) and $6 \mathrm{~dB}$ ( $80 \%$ white noise). One clear conclusion stemming from these results is that a large margin between white and quantization noise increases the yield for any design aggressiveness. The three sets of simulations also show that, for a more aggressive NTF (as is the case with the target DRwn of $102 \mathrm{~dB}$ ), the yield sensitivity to mismatch is larger and even a large noise margin cannot completely cover the DR decrease induced by changes of NTF in-band shape. The set of simulations for DRwn of $102 \mathrm{~dB}$ are actually pushing to the limit the aggressiveness of the fourth-order NTF designed for single-bit, single-loop $\Sigma \Delta$ ADCs, the $6 \mathrm{~dB}$ noise 


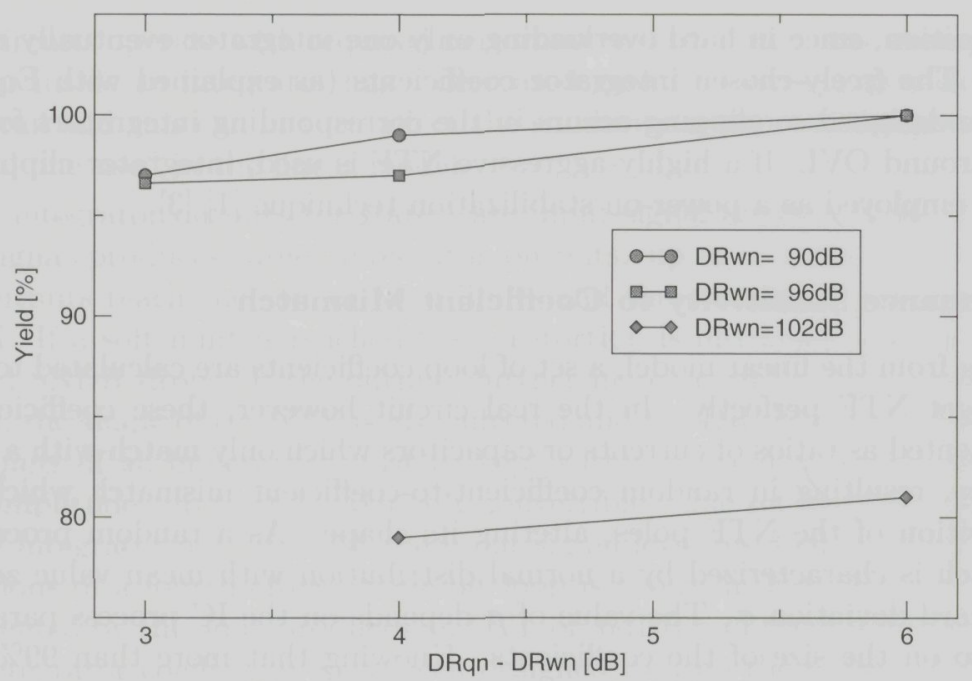

Figure 2.12: Yield of a fourth-order, single-bit $\Sigma \Delta A D C$ for different in-band margins between the circuit white-noise and quantization noise powers

margin being the maximally-available for this design at OSR $=64$ (highest $D R q n$ is $108 \mathrm{~dB})$.

The technique of designing the white noise to dominate over quantization noise inside the signal bandwidth is also convenient for covering the low-frequency tones produced especially in single-loop, low-order $\Sigma \Delta$ ADCs. This is a design goal itself in ADCs targeted toward audio applications. References on how to reduce tonal behavior are available in published literature [1] [6].

\subsubsection{Cascaded Topologies}

Cascaded $\Sigma \Delta$ ADC designs consist of a chain of $\Sigma \Delta$ ADC loops, only the first loop processing the input signal while the following ones process the quantization noise of the previous loop in the chain, respectively. In this way, the cascaded $\Sigma \Delta$ ADC designs maximize both the DR by using highly-aggressive (high cutoff frequency) noise shaping and the OVL by only applying the full input signal to a low-order loop placed as the first stage in the cascade. Cascaded designs are convenient in applications where a de-coupling between the increase in DR and decrease in peak SNDR is wanted at a fixed OSR, as is the case with broadband ADCs that reach into technology-limited OSR values. The price to pay for this flexibility is a higher sensitivity to process-induced mismatches and to other circuit non-idealities introducing errors between calculated and actually implemented $\Sigma \Delta$ loop coefficients. This sensitivity is an effect of the topology 
of such designs which count on a combination of analog circuitry and digital filters to cancel low-order shaped quantization noise.

\section{Digital Cancellation of Quantization Noise}

One possible topology for a fifth-order, three-loop cascade $\Sigma \Delta \mathrm{ADC}$ is given in Fig. 2.13. It consists of a first loop, taking the input signal $X(z)$ and generating the digital stream $Y_{1}(z)$ which contains a delayed version of $X(z)$

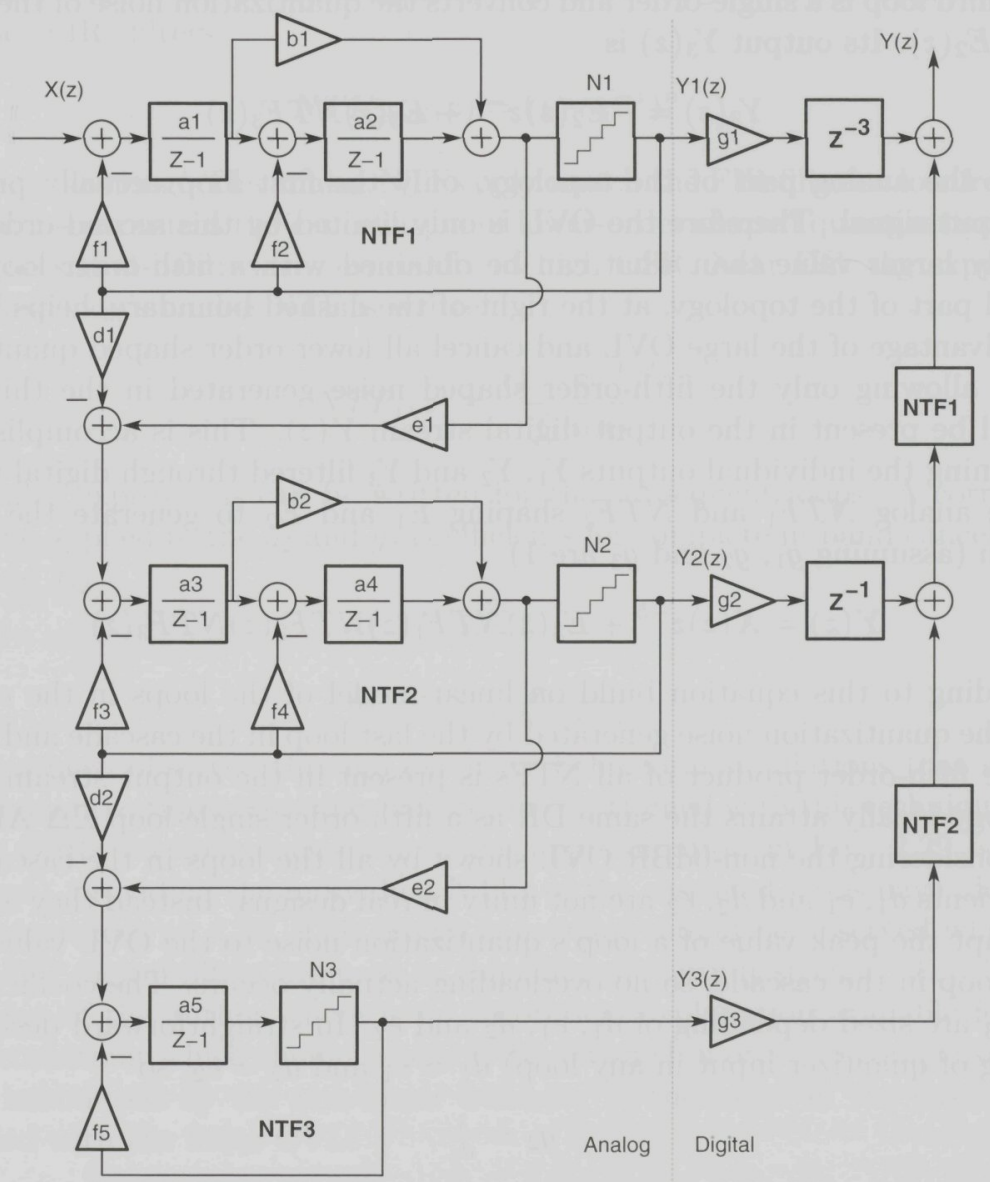

Figure 2.13: Fifth-order, 3-loop cascade $\Sigma \Delta A D C$ complete with digital processing for cancellation of low-order shaped quantization noise 
and quantization noise $E_{1}(z)$ shaped by the second-order $N T F_{1}$

$$
Y_{1}(z)=X(z) z^{-2}+E_{1}(z) N T F_{1}(z)
$$

The second loop, also a second-order in this case, converts the quantization noise generated by the first quantizer $E_{1}(z)$. For the purpose of this demonstration based on linear models the coefficients $d_{1}$ and $e_{1}$ can be assumed to be unity without loss of generality. Under these conditions the output of the second loop, $Y_{2}(z)$, is

$$
Y_{2}(z)=-E_{1}(z) z^{-2}+E_{2}(z) N T F_{2}(z)
$$

The third loop is a single-order and converts the quantization noise of the second loop, $E_{2}(z)$. Its output $Y_{3}(z)$ is

$$
Y_{3}(z)=-E_{2}(z) z^{-1}+E_{3}(z) N T F_{3}(z)
$$

So, in the analog part of the topology, only the first loop actually processes the input signal. Therefore the OVL is only limited by this second-order loop, a likely larger value than what can be obtained with a fifth-order loop. The digital part of the topology, at the right of the dashed boundary, helps keeping the advantage of the large OVL and cancel all lower-order shaped quantization noise, allowing only the fifth-order shaped noise generated in the third loop to still be present in the output digital stream $Y(z)$. This is accomplished by combining the individual outputs $Y_{1}, Y_{2}$ and $Y_{3}$ filtered through digital replicas of the analog $N T F_{1}$ and $N T F_{2}$ shaping $E_{1}$ and $E_{2}$ to generate the output stream (assuming $g_{1}, g_{2}$ and $g_{3}$ are 1 )

$$
Y(z)=X(z) z^{-5}+E_{3}(z) N T F_{1}(z) N T F_{2}(z) N T F_{3}(z)
$$

According to this equation build on linear-model of the loops in the cascade, only the quantization noise generated by the last loop in the cascade and shaped by the fifth-order product of all NTFs is present in the output stream, so the topology ideally attains the same $\mathrm{DR}$ as a fifth-order single-loop $\Sigma \Delta \mathrm{ADC}$.

Considering the non-0dBR OVL shown by all the loops in the cascade, the coefficients $d_{1}, e_{1}$ and $d_{2}, e_{2}$ are not unity in real designs. Instead they are sized to adapt the peak value of a loop's quantization noise to the OVL value of the next loop in the cascade, so no overloading actually occurs. The coefficients $g_{2}$ and $g_{3}$ are sized depending of $d_{1}, e_{1}, d_{2}$ and $e_{2}$. In straightforward designs (no scaling of quantizer input in any loop) $d_{1}=e_{1}$ and $d_{2}=e_{2}$, so

$$
\begin{aligned}
& g_{2}=\frac{1}{d_{1}} \\
& g_{3}=\frac{1}{d_{1} d_{2}}
\end{aligned}
$$

$g_{1}$ is only different from unity if there are differences in the reference level of the first and the subsequent loops.

28 Systematic Design of $\Sigma \Delta$ ADCs 


\subsection{Sigma-Delta ADCs with Discrete-Time Loop Filters}

\section{Practical NTF Design for Cascaded Loops}

The advantage of cascaded designs over single-loop designs is that a much more aggressive overall NTF is implemented as the product of multiple, lower-order NTFs without sacrificing stability and OVL. This is theoretically accomplished by re-combining the digital output streams of the cascaded loops by using digital filters (the blocks marked NTF1, NTF2 in Fig. 2.13) and delays. To simplify the digital part and reduce sensitivity to analog mismatches, only chains of accumulators are typically used instead of the complete, infinite impulse response (IIR) NTF filters $N T F_{1}$ and $N T F_{2}$ [7]. When the topology is so implemented, the analog NTFs are matched against digital NTFs which are finite impulse response (FIR) filters,

$$
N T F_{\text {ideal }}(z)=\left(1-z^{-1}\right)^{n}
$$

for order $n$ shaping. The analog NTFs only match the FIR filter at low frequencies, which guarantees the cancellation of lower-order shaped quantization noise inside the signal bandwidth, especially at high OSR. Near DC, the expression of the first loop's analog NTF is written

$$
N T F_{1}(z) \approx \frac{\left(1-z^{-1}\right)^{2}}{f_{1} a_{1} a_{2}}
$$

and similar expressions can be written for the subsequent loops. A correction should be applied to the $g_{2}$ and $g_{3}$ coefficients for complete in-band cancellation of $E_{1}$ and $E_{2}$

$$
\begin{aligned}
& g_{2} \longrightarrow \frac{g_{2}}{f_{1} a_{1} a_{2}} \\
& g_{3} \longrightarrow \frac{g_{3}}{f_{1} f_{3} a_{1} a_{2} a_{3} a_{4}}
\end{aligned}
$$

It has been observed through extensive behavioral simulations that a good in-band quantization noise cancellation can be attained with this technique even for low OSRs (at frequencies where the approximation in Eq. 2.29 is more erroneous), providing the individual NTFs of the cascaded loops have identical peak magnitudes at $f_{s} / 2$. This design constraint should be observed whenever simple FIR digital filters are used to calculate the output stream $Y(z)$. To the knowledge of the author, there is no theoretical explanation available for this design constraint which should probably be satisfied in order to compensate for effects introduced by the non-linear behavior of the loops in the cascade, as explained with the finite OVL.

\section{Performance Sensitivity to Coefficient Mismatch}

As it has been explained with the single-loop designs, the loop coefficients actually implemented in the final design are ratios of currents or capacitors and they 
never have the exact designed values. Instead, mismatches showing a normal distribution with zero mean and standard deviation $\sigma$ are introduced by the IC process used for implementation. The effect of such mismatches is to change the shape of the implemented NTF, thus placing more quantization noise at low frequencies (in the worst case).

In cascaded designs, the individual NTFs of all the loops in cascade are affected by mismatch. In the same time, the digital replicas of the (designed) analog NTFs are not affected by mismatch, hence a difference between the amplitude of the two filters appears and the lower-order shaped quantization noise is not completely canceled. In Fig. 2.13, differences between the analog $N T F 1, N T F 2$ and their digital replicas propagate a part of the quantization noise of the first two loops, $E_{1}(z)$ and $E_{2}(z)$, to the output digital stream, $Y(z)$

$$
\begin{aligned}
Y(z)= & X(z) z^{-5}+ \\
& E_{1}(z)\left(N T F_{1}^{\text {digital }}(z)-N T F_{1}^{\text {analog }}(z)\right) z^{-3}+ \\
& E_{2}(z)\left(N T F_{2}^{\text {digital }}(z)-N T F_{2}^{\text {analog }}(z)\right) N T F_{1}^{\text {digital }}(z) z^{-1}+ \\
& E_{3}(z) N T F_{1}(z) N T F_{2}(z) N T F_{3}(z)
\end{aligned}
$$

The non-ideal expression of $Y(z)$ contains the input signal $X(z)$, the fifth-order shaped quantization noise of the third loop $E_{3}(z)$, but also the second-order shaped $E_{1}(z)$ and fourth-order shaped $E_{2}(z)$ multiplied with corresponding mismatches between their digital and analog NTFs. For identical mismatches between digital and analog NTFs, $E_{2}(z)$ is also multiplied with the second-order $N T F_{1}^{\text {digital }}(z)$ compared to $E_{1}(z)$ which directly connects to the output. Since $E_{1}(z)$ is only second-order shaped, it is the dominant error term in the non-ideal output.

Even if the simplified digital implementation is used and the analog NTFs are approximated as shown in Eq. 2.29, there is still the mismatch between the analog products $f_{1} a_{1} a_{2}, f_{3} a_{3} a_{4}$ and their digital counterparts $g_{2}, g_{3}$. This difference shifts the analog NTFs compared to their digital replicas at low frequencies (gain error only).

Compared to single-loop designs, the effect of $\Sigma \Delta$ loop coefficient mismatch is more important in cascaded designs since it affects the cancellation of the lower-order shaped noise. The production yield is the measure of design's sensitivity to coefficient mismatch. Considering a given mismatch distribution, the yield can be increased by reducing the power of the dominant error, in this case $E_{1}(z)$. This is accomplished by designing the number of bits in the first quantizer $N 1$ so the power of $E_{1}(z)$ leaking to the output does not significantly reduce the yield. For the topology in Fig. 2.13 operated at OSR $=16$ and having 3 bits in the second and third quantizers, also 3 bits are sufficient in the nominal case if the target DR is $96 \mathrm{~dB}$. However, for a coefficient mismatch characterized by $3 \sigma=0.5 \%$ (Fig. 2.14), 4 bits should be designed in the first quantizer to 


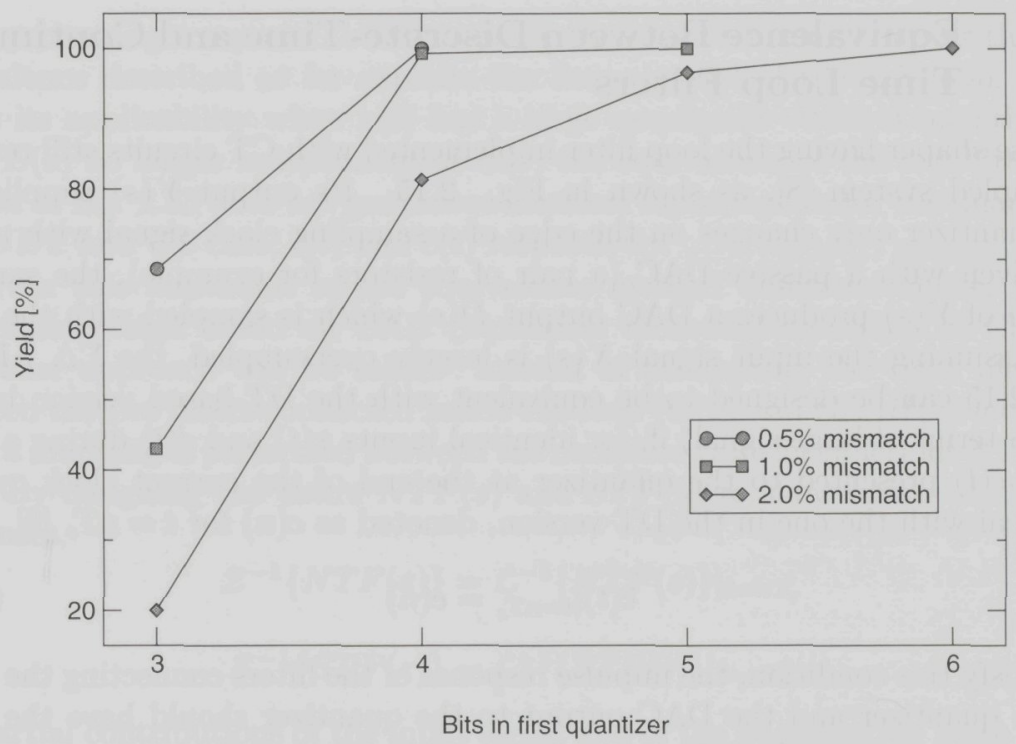

Figure 2.14: Yield of a fifth-order, three-loop cascaded $\Sigma \Delta A D C$ with 3 bits in the last two quantizers as a function of the number of bits in the first quantizer

keep the yield at $100 \%$. The situation is similar for $3 \sigma=1.0 \%$ even if, with 3 bits in the first quantizer, the yield drops to $43 \%$ from $68 \%$. Only with 5 bits in the first quantizer is it possible to maintain good yield when $3 \sigma=2.0 \%$.

\subsection{Sigma-Delta ADCs with Continuous-Time Loop Filters}

Not only discrete-time filters can be used to implement the loop transfer functions in $\Sigma \Delta$ ADCs. The two signal applied to the loop filter, the input signal and the DAC feedback signal, can be assumed to be stable during one clock cycle. Under these conditions, the loop filter can be implemented with continuous-time (CT) filters without changing the overall behavior of the ADC. The design of the CT loop filter can be done by direct synthesis or by deriving it from a DT filter. The second method opens the possibility of comparison between fundamental performance of DT- and CT-based $\Sigma \Delta$ ADCs and is therefore preferred in this systematic analysis. 


\subsubsection{Equivalence Between Discrete-Time and Continuous- Time Loop Filters}

A noise shaper having the loop filter implemented with CT circuits still remains a sampled system [8], as shown in Fig. 2.15. Its output $Y(s)$ supplied by the quantizer only changes on the edge of a sampling clock signal with period $T_{s}$. Even with a passive DAC (a pair of resistors for example), the sampled nature of $Y(s)$ produces a DAC output $D(s)$ which is sampled with the same $T_{s}$. Assuming the input signal $X(s)$ is largely oversampled, the $\Sigma \Delta$ ADC in Fig. 2.15 can be designed to be equivalent with the DT-based version in Fig. 2.1 (in terms of large signal) if, for identical inputs $x(t)$ and $d(t)$ during a clock cycle, $\hat{c}(t)$ presented to the quantizer at the end of the current clock cycle is identical with the one in the DT version, denoted as $c(n)$ for $t=n T_{s}[9]$,

$$
\left.\hat{c}(t)\right|_{t=n T_{s}}=c(n)
$$

To satisfy this condition, the impulse response of the filters connecting the input to the quantizer and the DAC output to the quantizer should have the same impulse response in the DT and the CT variants,

$$
\begin{gathered}
\mathcal{Z}^{-1}\{G(z)\}=\left.\mathcal{L}^{-1}\{\hat{G}(s)\}\right|_{t=n T_{s}} \\
\mathcal{Z}^{-1}\{G(z) H(z)\}=\left.\mathcal{L}^{-1}\{\hat{G}(s) \hat{H}(s)\}\right|_{t=n T_{s}}
\end{gathered}
$$

with $\mathcal{L}^{-1}\{\cdot\}$ being the inverse Laplace transform. With the equations above satisfied, the time-domain signal sampled by the quantizer is identical in the DT and the CT designs, and, ideally, time-domain simulation results obtained for the DT loop also apply to the CT loop. This is a major advantage since behavioral time-domain simulations for CT loops typically take more than 10

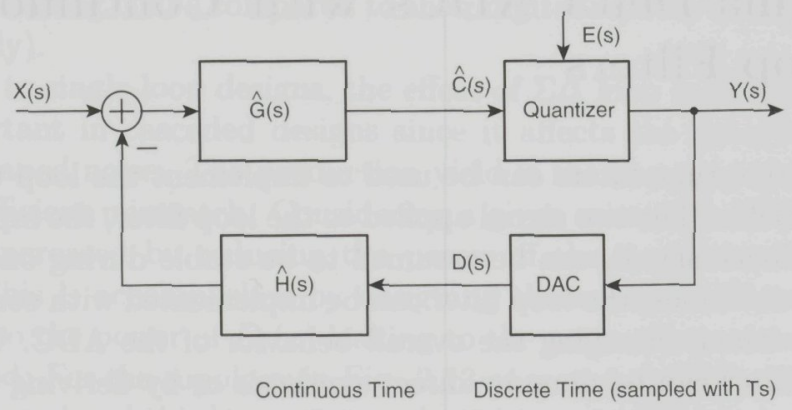

Figure 2.15: Noise shaper with continuous-time loop filter 


\subsection{Sigma-Delta ADCs with Continuous-Time Loop Filters}

times processing compared to DT loops [9]. It is worth noting however that the equivalence described so far exploits the linear model of the $\Sigma \Delta$ loop, which limits its applicability when $x(t)$ has a large amplitude. With a quantization noise $E(s)$ no longer independent of $X(s)$ the equivalence of the two filters described in Eq. 2.33 does not guarantee identical input signals for the quantizers in the DT and CT case, $c(t)$ and $\hat{c}(t)$ respectively.

Given this limitation in the equivalence method described above, in the systematic design based on CAD (numerical methods) described in Chapter 5 another approach will be taken. Instead of attempting the tight mapping between the two filters connected inside the loop $(G(z)$ and $G(z) H(z)$ individually), a mapping is performed between the complete transfer functions NTF and STF. By designing the entire $N \hat{T} F(s)$ and $S \hat{T} F(s)$ to show identical impulse responses,

$$
\begin{aligned}
& \mathcal{Z}^{-1}\{N T F(z)\}=\left.\mathcal{L}^{-1}\{N \hat{T} F(s)\}\right|_{t=n T_{s}} \\
& \mathcal{Z}^{-1}\{\operatorname{STF}(z)\}=\left.\mathcal{L}^{-1}\{S \hat{T} F(s)\}\right|_{t=n T_{s}}
\end{aligned}
$$

the partial contributions of the input signal and of the quantization noise to the quantizer input are identical in the two designs. This opens better comparison possibilities between the functioning of the DT and CT equivalent loops because the poles and zeros of the entire transfer functions are matched. No advantage is lost since the tight mapping does not actually guarantee identical time-domain behavior of the two designs for the entire input dynamic range.

The direct mapping of NTF and STF assumes a constant DAC output during one clock cycle. As the next sub-Section explains, return-to-zero (RTZ) DAC waveforms are needed in high-DR CT designs. RTZ DACs switch from zero to nominal value during any clock period and, with such DAC waveforms, the design method has to be adapted to consider the Laplace transform of the DAC impulse response.

\subsubsection{Effects of DAC Waveform Shape}

As opposed to DT loop filters, the value of the DAC output at any moment during a clock period is important in CT loop filters. The shape of the DAC pulse is part of the NTF expression (see Fig. 2.15)

$$
N T F(s)=\frac{1}{1+\hat{G}(s) \hat{H}(s) \hat{R}(s)}
$$

with $\hat{R}(s)$ being the transfer function of the DAC, as calculated by a Laplace transform of the DAC's impulse response. Hence the expression of $N T F(s)$ directly depends on the impulse response of the DAC, which is its output waveform. Changes of the DAC output waveform, inherent in any switching (and 
especially fast-switching) circuit, also affect noise shaping. This is a particularly sensitive issue in CT designs because the power density of the DAC pulse is high around the edges, making these designs more sensitive to non-ideal effects typically associated with level transitions in the DAC output.

\section{Intersymbol Interference}

Unequal rise and fall times in the DAC switching is one effect difficult to control by circuit techniques [10]. Considering a one-bit DAC used in CT $\Sigma \Delta$ loop, its output must switch between Iref and -Iref as decided by the loop quantizer on the previous sampling clock. Fig. 2.16 shows such a DAC output with non-equal rise and fall times, with the difference between rise and fall times being $\tau$. Two bit-stream sequences are analyzed, 101 and 110. If the rise and fall times are identical $(\tau=0)$, the charge delivered to the CT loop filter by both sequences is

$$
Q_{101}=Q_{110}=I_{\text {ref }} T_{s}
$$

where $T_{s}$ is the sampling clock period. With switching asymmetry however, the two waveforms change their charge content

$$
\begin{aligned}
& Q_{101}^{\tau}=I_{\text {ref }}\left(T_{s}-2 \tau\right) \\
& Q_{110}^{\tau}=I_{\text {ref }}\left(T_{s}-\tau\right)
\end{aligned}
$$

Since the bit-stream driving the DAC output contains both quantization noise and input signal, the charge delivered by the DAC becomes signal-dependent when $\tau \neq 0$. This effect introduces inter-symbol interference, so called because the charge delivered with one symbol (one bit in the output bit-stream) is not only dependent on the symbol itself but also on the previous symbol. Hence the different rise and fall times of the DAC output not only change the shape of the DAC pulse, affecting the NTF and therefore the noise shaping, but also makes

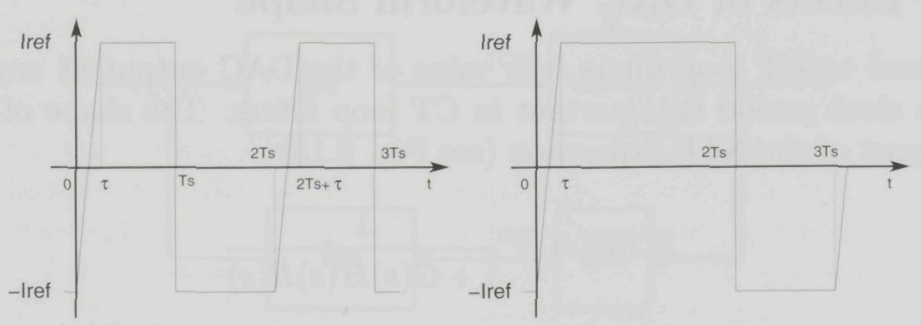

Figure 2.16: DAC waveforms with $\tau$ asymmetry between rise-time and fall-time, for "101"(left) and "110"(right) bit sequences

\section{Systematic Design of $\Sigma \Delta$ ADCs}




\subsection{Sigma-Delta ADCs with Continuous-Time Loop Filters}

the charge supplied by the DAC on each clock cycle to be signal-dependent. Signal dependency of the feedback charge puts harmonic distortion in the conversion result, reducing integral non-linearity (INL), or, in $\Sigma \Delta \mathrm{ADC}$ terms, the peak SNDR.

From Eqs. 2.37, the magnitude of this effect is expected to rise with increased DAC switching asymmetry (value of $\tau$ ). However, this magnitude can only be analytically estimated for first and second-order $\Sigma \Delta$ loops where the output symbol densities can be calculated [10]. Behavioral simulations must be run for higher-order loops, measuring the deviation of the peak SNDR from the ideal value obtained for $\tau=0$. Such results are shown in Fig. 2.17 for a fifth-order, single-bit $\Sigma \Delta$ loop with distributed feedbacks and feedforwards which attains a quantization-noise limited DR of $106 \mathrm{~dB}$ (with round symbols on the graph). The OVL is $-6 \mathrm{dBR}$, hence a peak SNDR of $100 \mathrm{~dB}$ can be reached. This value is only obtained if the rise-fall asymmetry is less than $0.0001 \%$ from the clock period $\left(\tau / T_{s} \leq 10^{-6}\right)$. Any value larger than that introduces a loss of peak SNDR by both in-band quantization noise leakage (at small $\tau$ values, actually reducing DR) as well as harmonic distortion (at large $\tau$ values). The SNDR is reduced by as much as $40 \mathrm{~dB}$ for $\tau / T_{s}=10^{-3}$, which only seems to be a hard-to-reach error value. For a CT $\Sigma \Delta$ running at $1 \mathrm{GHz}, T_{s}=1 n s$ and $\tau$ should then be $1 p s$ only to get $40 \mathrm{~dB}$ SNDR loss. At low $\tau$ values, where the

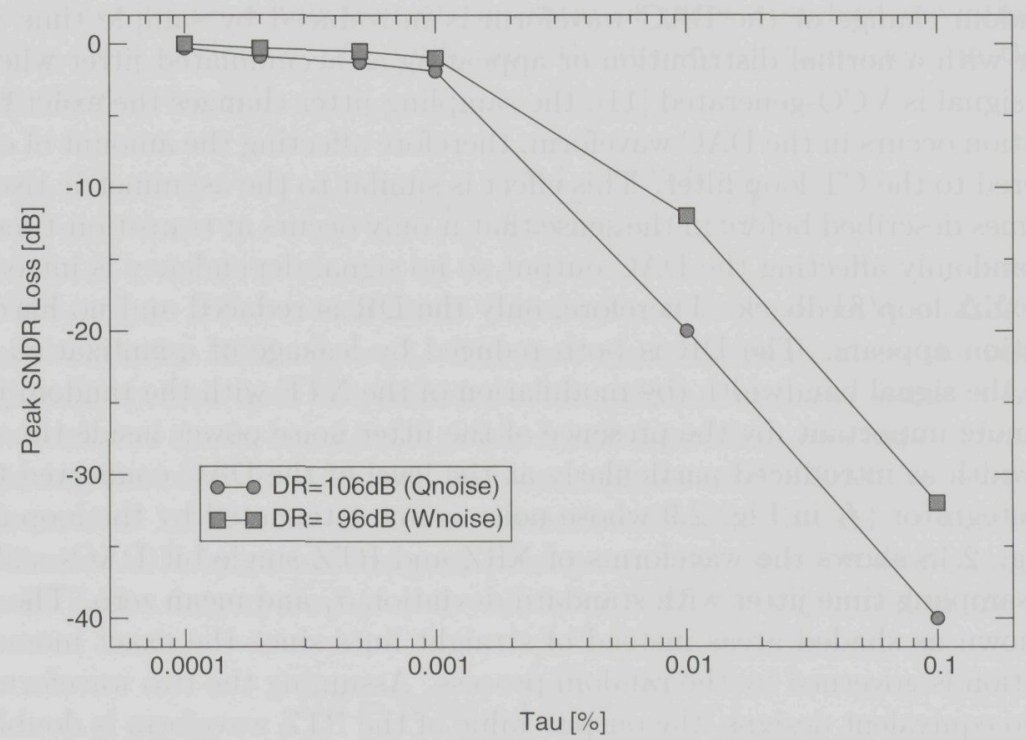

Figure 2.17: Peak SNDR loss due to ISI in a fifth-order single-loop CT $\Sigma \Delta$ $A D C$ with and without dominant in-band white noise 


\section{Architecture-Level Analysis of Sigma-Delta ADCs}

main error is DR reduction, the effect of asymmetric DAC pulse can be reduced by allowing the white (circuit) noise to dominate over the quantization noise inside the signal bandwidth. For the same design, a second curve shows the loss of SNDR if the white noise sets a target DR of only 96dB (margin of $10 \mathrm{~dB}$ to quantization noise power). By so designing the $\Sigma \Delta$ ADC the SNDR loss is reduced at small $\tau$ values, but not at large values where the harmonic distortion dominates over the noise floor.

One method to correct the effects of DAC waveform asymmetry is to employ a return-to-zero (RTZ) DAC output, which, during each clock cycle, switches according the quantizer decision for part of the time and resets to zero for the rest. In this way, independent of the quantizer decision, both a rising and a falling edge appear in the DAC pulse. Furthermore, the inter-symbol interference is reduced because, no matter the previous quantizer decision, a new DAC output starts from the same, reset, value. The use of RTZ DACs obviously changes the DACs transfer function $\hat{R}(s)$ (Eq. 2.35), hence it requires re-calculation of NTF denominator coefficients to keep the position of its poles [9]. A RTZ DAC waveform is shown in Fig. 2.18, where it is used to demonstrate why a RTZ design is more sensitive to phase jitter of the sampling clock compared to NRZ designs.

\section{Clock Jitter}

A random change of the DAC waveform is introduced by sample time jitter. Either with a normal distribution or appearing as accumulated jitter when the clock signal is VCO-generated [11], the sampling jitter changes the exact time a transition occurs in the DAC waveform, therefore affecting the amount of charge delivered to the CT loop filter. This effect is similar to the asymmetric rise- and fall-times described before in the sense that it only occurs at transition-time, but it is randomly affecting the DAC output so no signal-dependency is introduced in the $\Sigma \Delta$ loop feedback. Therefore, only the DR is reduced and no harmonic distortion appears. The DR is both reduced by leakage of quantization noise inside the signal bandwidth (by modulation of the NTF with the random jitter) and, more important, by the presence of the jitter noise power inside the signal bandwidth as introduced particularly at the level of the DAC connected to the first integrator ( $f_{1}$ in Fig. 2.9 whose noise is not attenuated by the loop gain).

Fig. 2.18 shows the waveforms of NRZ and RTZ single-bit DACs suffering from sampling time jitter with standard deviation $\sigma_{j}$ and mean zero. The edges are shown as shaded areas instead of straight lines since the exact moment of transition is governed by the random process. Assuming the two waveforms are for two equivalent designs, the output value of the RTZ waveform is doubled (if RTZ duration is half the clock period, RTZ duty cycle 50\%) to supply the same amount of charge with one symbol. From the comparison of the two waveforms it can be seen that a NRZ DAC is affected by this random effect only when a 

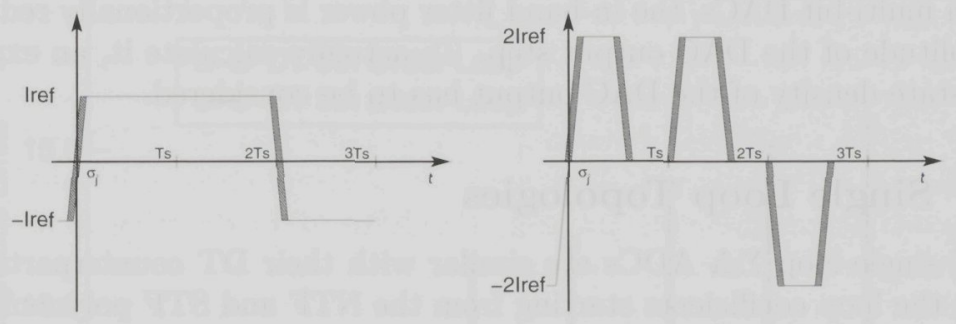

Figure 2.18: DAC waveforms with $\sigma_{j}$ clock jitter for "110" pattern, implemented with NRZ (left) and RTZ (right)

transition occurs (which introduces a signal-dependency of the jitter power in the DAC output) while the RTZ DAC takes 4 times more jitter power because there are two transitions during any clock cycle and the output power is doubled during the active phase. Hence it can be concluded that the loss of DR is signaldependent for NRZ DACs [11] while it is signal-independent but 6dB larger for the RTZ DACs with $50 \%$ duty cycle.

For single-bit DACs the DR value when clock jitter power dominates inside the signal bandwidth has been calculated for zero-mean, normally-distributed (white) jitter to be a function of the ratio of jitter standard deviation and sampling clock period, $\sigma_{j} / T_{s}$, OSR, and the density of transitions in the bitstream output, $\sigma_{\delta y}[11]$

$$
D R=10 \log _{10}\left(\frac{1}{2 \sigma_{\delta y}^{2}} \frac{O S R}{\left(\sigma_{j} / T_{s}\right)^{2}}\right)
$$

For NRZ, $\sigma_{\delta y}^{2}=2.8$ for zero input (maximum number of DAC output transitions), while for RTZ $\sigma_{\delta y}^{2}=8$. The last result is identical to the one estimated in [2] (which does not consider a bit-stream transition density) since in RTZ waveforms the in-band jitter power is not dependent on symbol transition. Comparing the result in 2.38 with the one in [2] for a NRZ DAC however yields a difference of $1.4 \mathrm{~dB}$ related to the transition density. Hence, estimating the jitter-limited DR by considering an average number of bit-stream transitions and a RTZ-like duty-cycle $\delta(\delta=0 \cdots 1,1$ for NRZ), as explained in [2]

$$
D R=10 \log _{10}\left(\frac{\delta^{2}}{4} \frac{O S R}{\left(\sigma_{j} / T_{s}\right)^{2}}\right)
$$

is accurate enough for usual applications where the target DR is larger than $60 \mathrm{~dB}$ (10 bits resolution). 
With multi-bit DACs, the in-band jitter power is proportionally reduced by the amplitude of the DAC output step. To actually calculate it, an expression for the state density of the DAC output has to be considered.

\subsubsection{Single Loop Topologies}

The CT single-loop $\Sigma \Delta$ ADCs are similar with their DT counterparts in calculating the loop coefficients starting from the NTF and STF polynomials, deriving the loop gains and consequently designing each integrator's performance in terms of noise and distortion. They also show similar sensitivity to loop coefficient mismatch, but on top of that the CT loops bring in a performance limitation related to accentuated non-linear behavior, which decreases the OVL and stable NTF limits. Another CT loop issue, related to the method of implementing the loop coefficients on ICs, is also discussed in this sub-Section.

\section{Fundamental Performance Limitation Compared to DT Loops}

It has been mentioned before that a mapping of complete NTF and STF from realizable DT loop filters to $\mathrm{CT}$ equivalents opens the possibility to compare the performance of two equivalent implementations of virtually the same design. "Virtually" highlights the fact that an equivalence is only valid when the linear model holds in both cases, which is not true with large input signals. Therefore, it is important to analyze the performance of the two $\Sigma \Delta$ ADCs, with equivalent DT and CT loop filter respectively, as independent designs and compare the results from time-domain simulations which account for the non-linear loop transfer.

The range of usable NTF aggressiveness (as defined by the lower and upper limits of peak NTF magnitude yielding a stable NTF) has been found to vary with the order of the loop, the number of bits in the quantizer, as well as with the nature of the loop filter, DT or CT. This is proven in Fig. 2.19 for a set of fifth-order, single-loop modulators, with the number of quantizer bits ranging from 1 to 8 . For any number of bits in the quantizer, the CT equivalents cannot be designed as aggressive as the DT designs. Also, the range of usable NTFs increases very fast with the number of quantizer bits for DT designs but very slow for the CT equivalents. These results are valid for NRZ, CT DAC pulses. Independent simulations using RZ DAC pulses proved to yield results closer to DT designs, intuitively placing the cause of the effects explained for the NRZ designs in the DAC pulse shape.

\section{Sensitivity to Initial Accuracy of Implemented Coefficients}

One issue which was not present with DT loop design is the initial accuracy of all loop coefficients implemented in the final circuit. As opposed to DT loops, 


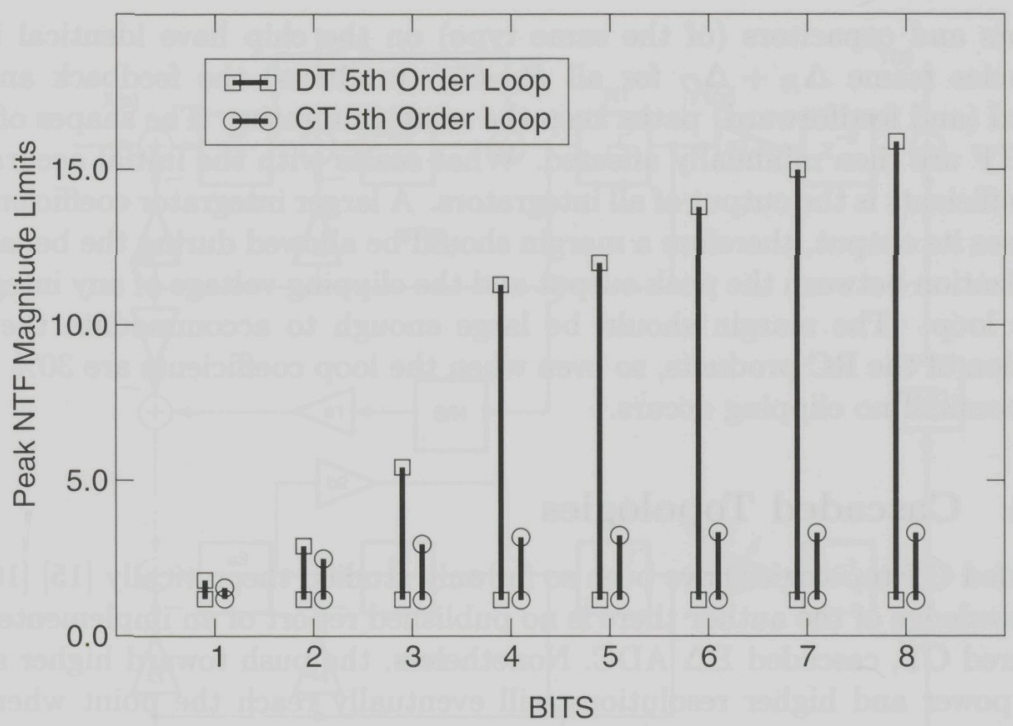

Figure 2.19: Peak NTF magnitude ranges as function of quantizer bits for DT and $C T$ loops with $O R D E R=5$

where all coefficients are implemented as ratios of either capacitors or currents [3] [12] [13], in CT loops the typical coefficient implementation is a resistorcapacitor $(R C)$ product [14]. Other methods include current-capacitor ratios $(I / C)$, especially for DACs, and transconductance-capacitor ratios $\left(G_{m} / C\right)[2]$. All these CT coefficients ultimately show an initial accuracy only as good as an $\mathrm{RC}$ product, with the resistor and the capacitor integrated on a chip. If no calibration is available for the $\mathrm{RC}$ product [15], the two on-chip passive components $R_{I C}, C_{I C}$ show independent spreads from their designed values $R_{0}, C_{0}$

$$
\begin{aligned}
& R_{I C}=R_{0}\left(1+\Delta_{R}\right) \\
& C_{I C}=C_{0}\left(1+\Delta_{C}\right)
\end{aligned}
$$

with $\Delta_{R}$ and $\Delta_{C}$ being two process-dependent, uncorrelated errors with standard deviations up to $5 \%$. Under these conditions the RC product will vary as much as

$$
R_{I C} C_{I C}=R_{0} C_{0}\left(1+\Delta_{R}\right)\left(1+\Delta_{C}\right) \approx R_{0} C_{0}\left(1+\Delta_{R}+\Delta_{C}\right)
$$

which gives a standard deviation of the RC product in the range of $10 \%$. For a normal distribution, this means practically all RC products are within $30 \%$ in error from their nominal value.

The method to cope with this spread of loop coefficients is based on the assumption that spread is the same across a processed wafer. Since all the 
resistors and capacitors (of the same type) on the chip have identical initial accuracies (same $\Delta_{R}+\Delta_{C}$ for all $R_{I C} C_{I C}$ products) the feedback and the forward (and feedforward) paths keep the same gain ratios. The shapes of NTF and STF are then minimally affected. What scales with the initial accuracy of the coefficients is the output of all integrators. A larger integrator coefficient also increases its output, therefore a margin should be allowed during the behavioral optimization between the peak output and the clipping voltage of any integrator in the loop. The margin should be large enough to accommodate the peak deviation of the RC products, so even when the loop coefficients are $30 \%$ larger than nominal no clipping occurs.

\subsubsection{Cascaded Topologies}

Cascaded CT topologies have been so far only studied theoretically [15] [16]. To the knowledge of the author there is no published report of an implemented and measured CT, cascaded $\Sigma \Delta$ ADC. Nonetheless, the push toward higher speed, lower power and higher resolutions will eventually reach the point where CT cascaded designs will be implemented, therefore this systematic analysis of $\Sigma \Delta$ ADCs also includes a brief look at the issues likely to rise during the design of such architectures. One solution for digital cancellation of lower-order shaped quantization noise from $\mathrm{CT}$ loops is given and also a solution to reduce leakage of quantization noise at low frequencies due to loop coefficient (RC product) spread is explained.

\section{Digital Cancellation of Quantization Error}

Starting from the DT fifth-order cascade in Fig. 2.13, a CT equivalent is built (Fig. 2.20) by mapping the $N T F(z)$ for the three loops to their CT versions, $N \hat{T} F(s)$. This can be done under the assumptions explained with Eq. 2.32, which hold for the first loop with the slow-changing X(s) but do not apply for the second loop if a direct connection is made between its input and the input of the first quantizer. The latter node outputs a fast-changing signal, continuously integrated by the second loop. This fast-changing input breaks the assumption of a steady input signal under which the equivalence between the DT and the CT NTF has been developed, so the digital re-combination must also be re-designed. This digital re-design is time-consuming and depends on the particular architecture used [1] [16], so in this systematic design approach a sample and hold (the $S / H$ block) is inserted at the inputs of the second and third loops to hold the signal steady during on clock cycle. This turns the input signals of the higher-order loops into DT ones since the outputs of the first and second quantizers are also DT signals. The performance of the $S / H$ in terms of noise and harmonic distortion is attenuated by the loop gain of the first to integrators so the circuit does not add a design challenge or significant 


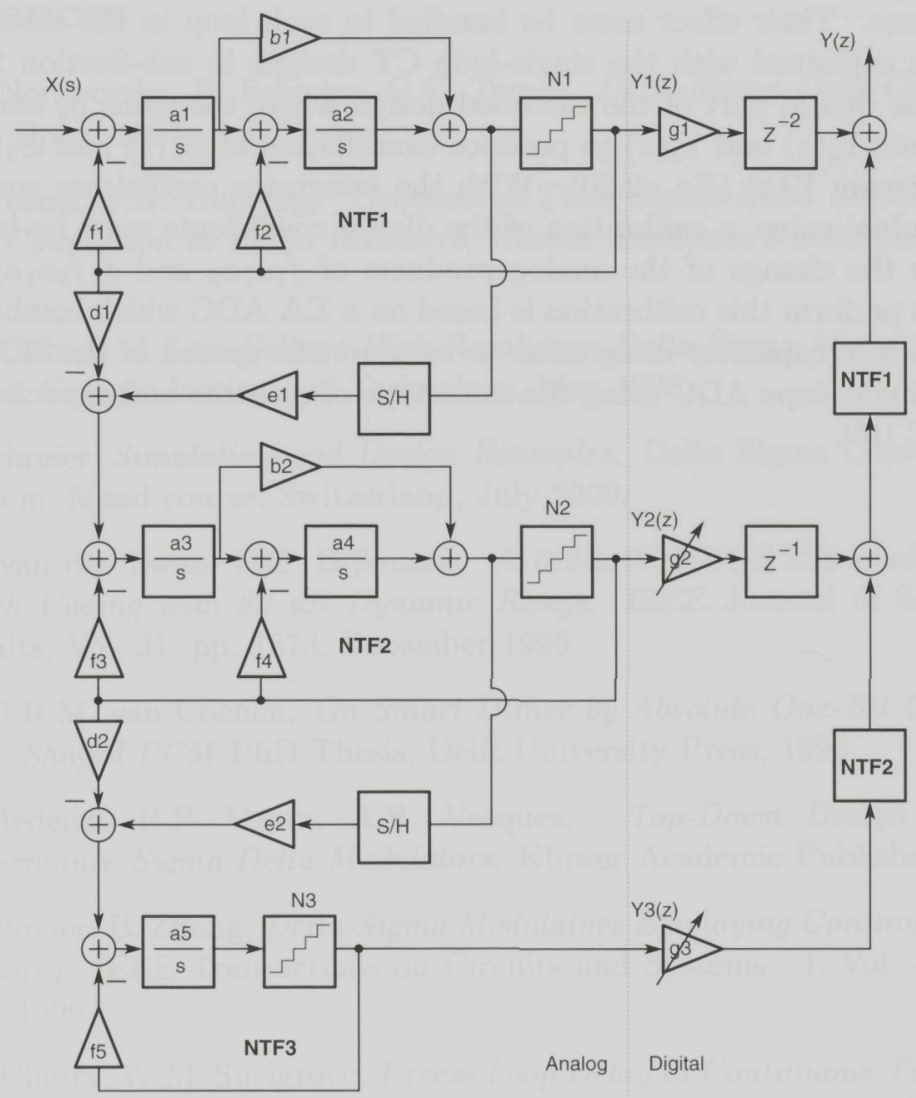

Figure 2.20: Fifth-order, 3-loop cascade, CT $\Sigma \Delta A D C$

power consumption. With the $S / H$ block inserted on the quantization noise path, the digital outputs of the cascaded CT loops can be re-combined in the same manner as the ones from the DT equivalents, using expressions of the DT $N T F 1$ and $N T F 2$ or simplified FIR filters as explained with Fig. 2.13. There are 2- and 1-clock delays ( $z^{-2}$ and $z^{-1}$ blocks) inserted at the outputs of the first and second loops to bring the three digital streams at the same phase delay compared to the input signal X(s).

\section{Effects of Initial Accuracy of Implemented Coefficients}

As it has been explained in sub-Section 2.3.3, the loop coefficients as implemented in the final circuit undergo process-induced spread which can show 
large values. Their effect must be handled in each loop in the cascade separately, as explained with the single-loop CT designs in sub-Section 2.3.3, but also in the digital part of the cascaded design where the gains $g_{2}$ and $g_{3}$ scale the outputs $Y_{2}(z)$ and $Y_{3}(z)$ to produce cancellation of $E_{1}(z)$ and $E_{2}(z)$ in the output stream $Y(z)$ (Eq. 2.30). With the integrator coefficients spread from their nominal value, a calibration of the digital coefficients must be introduced to match the change of the analog products of $f_{1} a_{1} a_{2}$ and $f_{1} f_{3} a_{1} a_{2} a_{3} a_{4}$. A circuit to perform this calibration is based on a $\Sigma \Delta$ ADC which combines a RC and capacitor/capacitor integration to measure the spread of the RC product, or on a single-slope ADC using the same type of resistors and capacitors as the $\Sigma \Delta$ ADC [15]. 


\section{References}

[1] S.R. Norsworthy, R. Schreier, G.C. Temes, Delta-Sigma Data Converters, IEEE Press, 1997.

[2] L. Breems, J.H. Huijsing, Continuous-Time Sigma-Delta Modulation for $A / D$ Conversion in Radio Receivers, Kluwer Academic Publishers, Boston, 2001.

[3] A.L. Coban, A Low-Voltage High-Resolution Delta-Sigma Modulator, PhD Thesis, Georgia Institute of Technology, May 1998.

[4] R. Schreier Simulation and Design Examples, Delta Sigma Converters for Telecom, Mead course, Switzerland, July 2000.

[5] E.J. van der Zwan, E.C. Dijkmans, A 0.2-mW CMOS $\Sigma \Delta$ modulator for Speech Coding with 80 dB Dynamic Range, IEEE Journal of Solid-State Circuits, Vol. 31, pp. 1873, December 1996.

[6] T.A.J.R.M. van Coenen, On Smart Dither by Absolute One-Bit Coding for Noise-Shaped PCM PhD Thesis, Delft University Press, 1996.

[7] F. Medeiro, B.P. Verdu, A.R. Vasquez, Top-Down Design of HighPerformance Sigma-Delta Modulators, Kluwer Academic Publishers, 1999.

[8] R. Schreier, B. Zhang, Delta-Sigma Modulators Employing Continuous-Time Circuitry, IEEE Transactions on Circuits and Systems - I, Vol. 43, No. 4, April 1996.

[9] J.A. Cherry, W.M. Snelgrove, Excess Loop Delay in Continuous-Time DeltaSigma Modulators, IEEE Transactions on Circuits and Systems - II, Vol. 46, No. 4, April 1999.

[10] J. Cherry, W.M. Snelgrove, Continuous-Time Delta-Sigma Modulators for High-Speed A/D Conversion, Kluwer Academic Publishers, 2000.

[11] J. Cherry, W.M. Snelgrove, Clock Jitter and Quantizer Metastability in Continuous-Time Delta-Sigma Modulators, IEEE Transactions on Circuits and Systems - II, Vol. 46, No. 6, June 1999.

[12] V. Peluso, M.S.J. Steyaert, W. Sansen, A 1.5-V-100- $\mu$ W $\Sigma \Delta$ Modulator with 12-b Dynamic Range Using the Switched-Opamp Technique, IEEE Journal of Solid-State Circuits, Vol. 32, No. 7, July 1997.

[13] C. Toumazou, G. Saether, M. Adams, Switched Current Circuit Design Techniques for Single-Bit, High Speed Sigma-Delta Modulators, IEE Colloquium on Oversampling Techniques and Sigma-Delta Modulation, March 1994. 
[14] R.W. Adams, Design and Implementation of an Audio $18 b$ Analog-toDigital Converter Using Oversampling Techniques Journal of Audio Engineering Society, Vol. 34, No. 3, March 1986.

[15] M. Ortmanns, F. Gerfers, Y. Manoli, On the Synthesis of Cascaded Continuous-Time $\Sigma \Delta$ Modulators IEEE International Symposium on Circuits and Systems, 2001, Vol. 5.

[16] Chi-Hung Lin, M. Ismail, Synthesis and Analysis of High-Order Cascaded Continuous-Time $\Sigma \Delta$ Modulators, IEEE International Conference on Electronics, Circuits and Systems, 1999.

[17] P.F. Ferguson, A. Ganesan, R.W. Adams, One Bit Higher Order SigmaDelta $A / D$ Converters, IEEE International Symposium on Circuits and Systems, 1990. 


\section{Discrete-Time Circuit Design}

Analog filters, ADCs and DACs that only process sampled analog signals can operate as discrete-time (DT) circuits controlled by the sampling clock. The DT circuits prove to be easy to simulate at behavioral level, as they only employ half-clock and 1-clock delays. Hence their functioning as linear systems can be modeled using Z-transforms, the same transforms used to analyze digital filters. These circuits often show advantages at transistor level, taking advantage of the higher switching speeds available in the deep sub-micron MOS transistors. Disadvantages of using discrete-time circuits include high transient currents on the supply lines and long transistor-level simulation times.

The main analog building block in $\Sigma \Delta$ ADCs is the first-order low-pass filter, or the integrator. Discrete-time integrators implement the Z-domain rational fraction

$$
H(z)=\alpha \frac{z^{-1}}{1-z^{-1}}
$$

where $z^{-1}$ is a one-clock delay and $\alpha$ a scaling factor, called the integrator coefficient. Three methods are currently used to build discrete-time integrators, all making heavy use of MOS switches to implement one-clock delays.

The first and most widely used is the switched-capacitor integrator, shown in Fig. 3.1 in its single-ended version. It consists of an opamp connected in closedloop with the integration capacitor $\mathrm{C} i$, which is used to accumulate charge, thus performing the low-pass function. For half the clock period (sampling phase) the input signal is sampled on the sampling capacitor Cs by closing the switches sw1 and sw3 and keeping sw2 and sw4 open. During the next half clock period, sw1 and sw3 are open and sw2 and sw4 are closed, thus connecting Cs to the charge-transfer node that is the negative opamp input. Because during this 


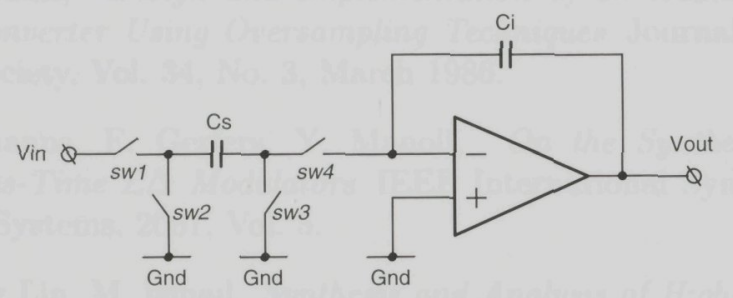

Figure 3.1: Switched-capacitor integrator

integrating phase the sampling capacitor $C s$ is connected between ground and the virtual ground node (held at virtual ground by the large opamp gain), all the charge stored in $\mathrm{Cs}$ is added to the charge of $\mathrm{Ci}$, modifying the output voltage accordingly. The transfer function can be written in Z-domain as

$$
H_{S C}(z)=\frac{V_{\text {out }}(z)}{V_{\text {in }}(z)}=\frac{C_{s}}{C_{i}} \frac{z^{-1}}{1-z^{-1}}
$$

Hence the integrator coefficient $\alpha$ is given by the ratio of the two capacitors, which is convenient from the point of view of yield optimization due to a relative mismatch of on-chip capacitors as low as $0.1 \%$ for a good layout.

Directly derived from the SC design is a design in which the sampling switch (sw1 in Fig. 3.1) is replaced by an opamp, as shown in Fig. 3.2. This switched opamp (SO) design [1] takes advantage of the presence of an opamp in front of any $\Sigma \Delta$ ADC integrator (either a buffer for input signal conditioning of the previous integrator in the loop) to eliminate the critical sampling switch sw1. The opamp OA1 can be switched on/off by the clock signal and operate in the same time as a SC amplifier (or integrator) and sampling switch. This

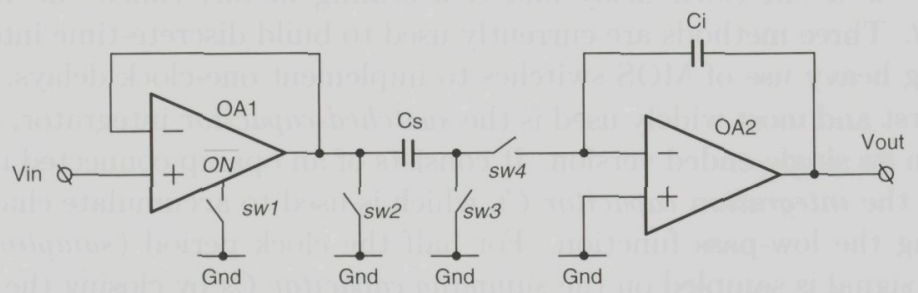

Figure 3.2: Switched-opamp integrator 
reduces the need for a linear floating switch but complicates the opamp design and operation. The SO designs avoid difficulties in driving a floating sampling switch in circuits operated at a supply voltage less than two times the threshold voltage of a MOS transistor. The transfer function of an $\mathrm{SO}$ integrator is similar to that of a $\mathrm{SC}$ integrator, even if each integrator simultaneously integrates and samples the output voltage on the next sampling capacitor. Opposite clock phases are used for consecutive integrators so there is no direct connection from the input of the loop filter to its output.

A completely different approach of designing DT integrators is the switchedcurrent (SI) method [2]. Instead of a voltage input and output, the SI integrator (shown in Fig. 3.3) has currents as input and output signals. During one clock phase the $s w 1$ and sw2 switches are closed, making the transistor M1 a MOS diode which samples by its gate-source capacitor the sum of the input and biasing currents minus the scaled previous output current sunk by M2. Switch $s w 4$ is also closed to make the one-clock-delayed output current available for the next integrator. Switch sw3 is open to keep the gate-source voltage of M2 and $M 3$ unchanged. During the second clock phase sw3 is closed and all the other switches are open, so the gate-source voltage of $M 2$ connected as a diode is sampled on the gate-source capacitance of M3. At this time the drain current of M2 is the integrated value of Iin. The transfer function is one of a DT integrator, written as

$$
H(z)=\frac{I_{\text {out }}(z)}{I_{\text {in }}(z)}=\alpha \frac{z^{-1}}{1-z^{-1}}
$$

if the ratio of the biasing currents and the MOS sizing are as shown in the schematic.

From the three design approaches outlined above, that of the SC circuits is most used in $\Sigma \Delta$ ADCs. These circuits are better suited for high-linearity

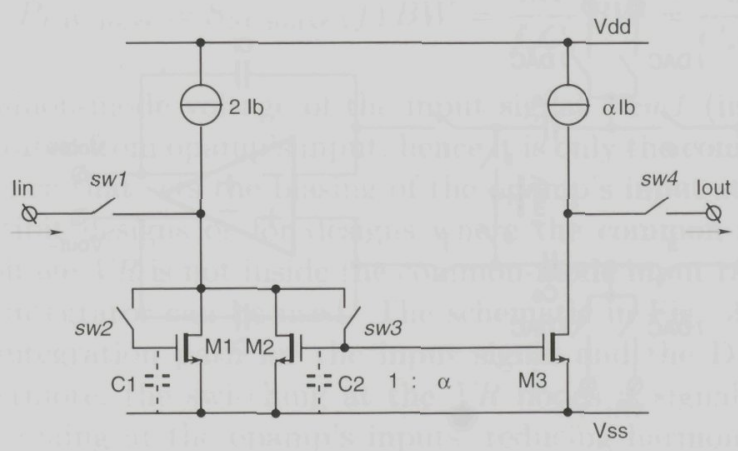

Figure 3.3: Switched-current integrator 
integrators than SO circuits. In SC circuits the opamp holds a closed loop, therefore all nonlinear effects introduced at its input are attenuated by the voltage gain. Compared to SI designs, the SC circuits show both better linearity and better coefficient matching, especially in high-speed CMOS processes where the output impedance of MOS transistors is significantly reduced. Because the focus of this chapter is the design of high-performance DT $\Sigma \Delta$ ADCs, only the $\mathrm{SC}$ circuits will be further analyzed. This analysis is also restricted to only differential designs for the same reason. The SC integrators are thoroughly analyzed in Section 3.1. In Section 3.2, a zero-delay SC amplifier needed to sum the feedforward coefficients is described. Both single-bit and multibit quantizers are covered in Section 3.3. The multibit DACs are explained in Section 3.4.

\subsection{Switched-Capacitor Integrators}

\subsubsection{Topologies for Summing Integrators}

At least the first integrator in a $\Sigma \Delta$ ADC loop has to integrate not only the input signal but also the pulses from a DAC, translating the decision of the quantizer into an analog signal to be fed back into the loop filter. The integrators presented in the introduction of this chapter only contain the signal path, since they were used to explain the circuit principles involved in various DT designs. This section gives a detailed overview of SC summing integrators which are used in $\Sigma \Delta$ ADC design.

Mostly used in single-bit $\Sigma \Delta$ ADCs is the differential integrator shown in Fig. $3.4 \mathrm{It}$ has a set of sampling capacitors $C s$ as the source of charge to be integrated on the $\mathrm{Ci}$ integration capacitors. During the sampling phase of the

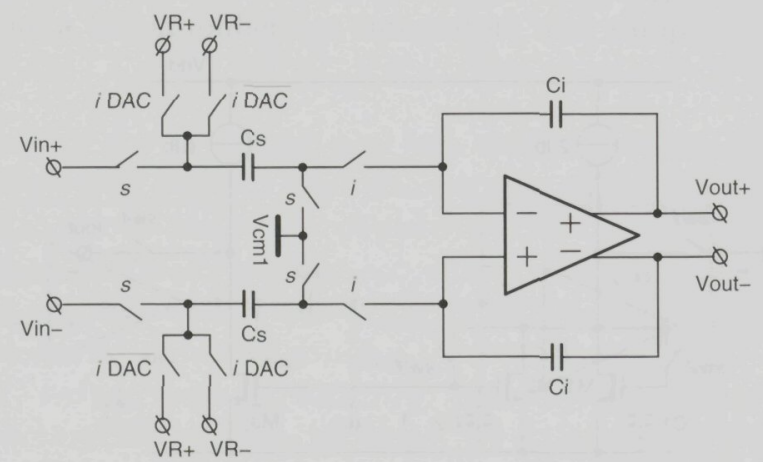

Figure 3.4: SC integrator with equal signal and DAC coefficients 


\subsection{Switched-Capacitor Integrators}

clock, the input signal Vin (either the input in the $\Sigma \Delta \mathrm{ADC}$ or the output of the previous integrator) charges the Cs capacitors. During the integration phase the $C s$ capacitors are connected to the virtual ground at the opamp inputs and to the reference voltage $V R$ according to the previous decision of the loop quantizer. If the previous decision is a logic 1 , it means that the output of the loop filter is too large and that the loop feedback should decrease it; hence the voltage Vin-VR is actually integrated as shown by the switching controls. By its construction, this integrator is fit for use in single-bit $\Sigma \Delta$ ADCs and if the input signal and the DAC pulses are multiplied by the same loop coefficient (the ratio of gains from the input voltage and the reference voltage to the integrator output is exactly 1 since the same sampling capacitor is used). This is generally the case with the first integrator in the loop if no conversion gain is implemented by the ADC [3]. The major advantage of this design is its simplicity: only a minimal number of switches is required, therefore the sampling noise is also minimized.

The noise introduced by the switching scheme is produced in the on-resistance of the switches (both sampling and integrating switches are uncorrelated noise sources) and filtered by the $C s$ capacitors [4]

$$
P_{\text {SCnoise }}=4 \int_{0}^{\infty} \frac{4 k T R_{O N}}{1+\left(2 \pi f R_{O N} C_{s}\right)} d f=\frac{4 k T}{C_{s}}
$$

resulting in a spectral density from DC to $f_{s} / 2$ (aliased by sub-sampling)

$$
S_{\text {SCnoise }}(f)=\frac{8 k T}{f_{s} C_{s}} \text {. }
$$

If an oversampling ratio of $O S R$ is assumed, the noise power inside the signal bandwidth can be calculated as the product of $S_{S C n o i s e}$ and the bandwidth since the noise is white

$$
P_{B W n o i s e}=S_{\text {SCnoise }}(f) B W=\frac{8 k T}{f_{s} C_{s}} B W=\frac{8 k T}{C_{s} O S R} .
$$

The common-mode voltage of the input signal Vcm1 (in Fig. 3.4) is completely separated from opamp's input, hence it is only the common-mode voltage of the reference that sets the biasing of the opamp's input stage.

For multibit designs or for designs where the common-mode value of the reference voltage $V R$ is not inside the common-mode input range of the opamp, a two-path integrator can be used. The schematic in Fig. 3.5 completely separates the integration path for the input signal and the DAC reference voltage. Furthermore, the switching at the $V R$ nodes is signal-independent, and so is the switching at the opamp's inputs, reducing harmonic distortion. The only signal-dependent switching is introduced by the use of physically different switches at the $V R$ nodes, but this has minimal effects due to their connection 


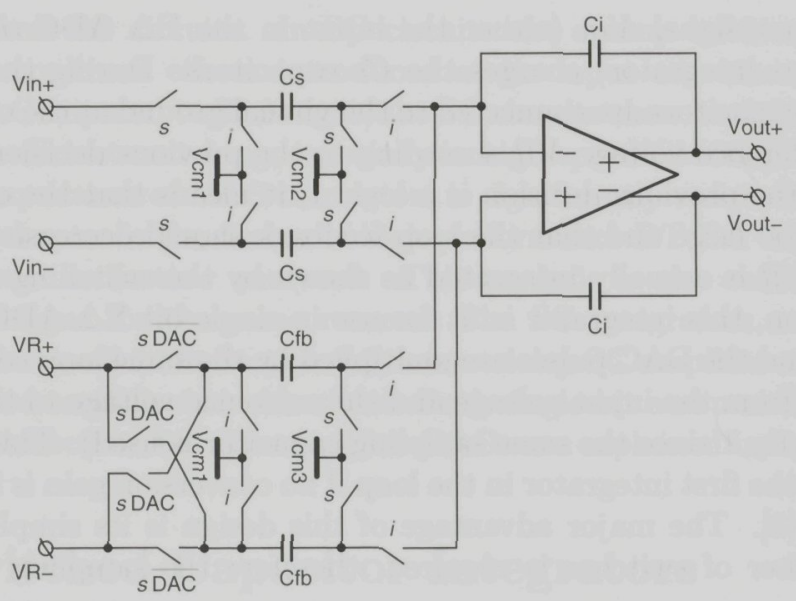

Figure 3.5: Summing SC integrator with different signal and DAC integration paths

to low-impedance nodes. Usually the $V R$ nodes are close to the supply rails and therefore complementary NMOS and PMOS switches can be used in the DAC. The noise power introduced inside the signal bandwidth by this switching scheme is

$$
P_{B W \text { noise }}=\frac{8 k T}{C_{s} O S R}\left(1+\frac{C_{f b}}{C_{s}^{2}}\right) .
$$

Due to the additional switching path, a larger total capacitance is required for the same noise budget.

A simpler method to build multibit DACs for SC integrators is to use a floating DAC capacitor, as shown in Fig. 3.6. In this case, the $C f b$ capacitor is differentially charged by the VR voltage so the common-mode voltage of the reference is not important. During the integration phase $C f b$ is connected between the two inputs of the opamp to be completely discharged; its charge is transferred to $\mathrm{Ci}$. One advantage is that the capacitor area is reduced by $50 \%$ since $C f b$ equates the two identical DAC capacitors in Fig. 3.5. A major disadvantage is the unbalanced connection of the parasitic capacitor $\alpha C f b$ associated in IC processing with any (a metal-metal, poly-poly or MOS) capacitor. The presence of the parasitic capacitor together with the signal-dependent connection to the reference voltage adds a signal-dependent load to the output common-mode control inside the opamp, which can translate in increased harmonic distortion by the finite common-mode to differential rejection. Therefore the use of this type of DAC is recommended only in the higher-order, less sensitive integrators in a $\Sigma \Delta \mathrm{ADC}$ loop filter. If a floating-capacitor approach is taken in high- 


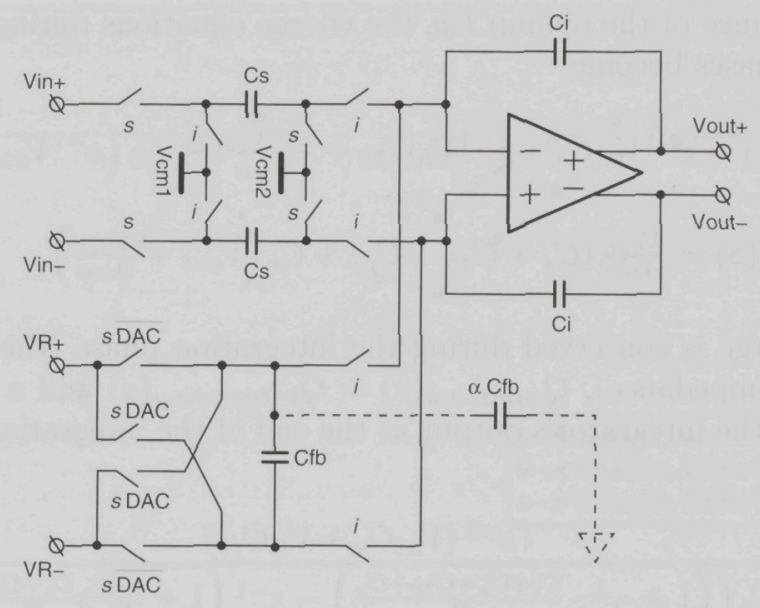

Figure 3.6: Summing SC integrator with floating DAC capacitor

performance integrators, $C f b$ must be implemented as two identical capacitors connected in series with their back side in the middle to obtain a symmetrical structure at the cost of an increased area. The common-mode voltage at the input of the opamp is only controlled by the common-mode voltage of the input signal, and the effectiveness of this control is also reduced by the presence of the parasitic $\alpha C f b$ capacitor.

\subsubsection{Effects of Circuit Non-idealities}

All circuits described in the previous section behave according to Eq. 3.1. However, they implement a DT integrator transfer function only if circuit nonidealities are neglected. But even if these non-idealities alter the function implemented in the final circuits, their effects on the overall conversion performance can be reduced by correct design of circuit components and signals. A few examples are given in this sub-Section.

\section{Finite, Nonlinear Amplifier Gain}

Amplifiers designed in CMOS processes suffer especially from a low value of the DC gain caused by reduced output impedance of MOS transistors. Even bipolar amplifiers have a finite DC gain and it affects the transfer function of the SC integrator through an incomplete discharge of the sampling and DAC capacitors, $C s$ and $C f b$ in Fig. 3.5, during the integration phase. The input capacitance of the opamp also becomes important in high-performance CMOS processes as these have a thinner gate oxide, and hence a larger gate capacitance. With the 


\section{Discrete-Time Circuit Design}

input capacitance of the opamp $C p$, the charge equations during sampling and integrating phases become

$$
\begin{aligned}
& Q_{\text {sampling }}(z)=z^{-1} V_{\text {in }} C_{s}+z^{-1} V_{R} C_{f b}+\frac{z^{-1} V_{\text {out }}}{A_{D C}}+C_{i}\left(z^{-1} V_{\text {out }}+\frac{z^{-1} V_{\text {out }}}{A_{D C}}\right) \\
& Q_{\text {integrating }}(z)=\frac{V_{\text {out }}}{A_{D C}}\left(C_{s}+C_{f b}+C_{p}\right)+C_{i}\left(V_{\text {out }}+\frac{V_{\text {out }}}{A_{D C}}\right) .
\end{aligned}
$$

Since the charge is conserved during the integration phase (the opamp inputs have infinite impedance), $Q_{\text {sampling }}(z)=Q_{\text {integrating }}(z)$ and a more accurate expression of the integrator's output at the end of the integration phase can be calculated:

$$
V_{\text {out }}=\frac{z^{-1}\left(V_{i n} C_{s}+V_{R} C_{f b}\right)}{C_{i}\left(\left(1+\frac{1}{A_{D C}} \frac{C_{i}+C_{s}+C_{f b}+C_{p}}{C_{i}}\right)-z^{-1}\left(1+\frac{1}{A_{D C}} \frac{C_{i}+C_{p}}{C_{i}}\right)\right)} .
$$

This expression can be placed in time-domain simulations of $\Sigma \Delta$ ADCs to show the impact of the finite amplifier DC gain on the conversion performance. Since Eq. 3.9 shows that a real integrator introduces both a gain and a pole error compared to the ideal integrator in Eq. 3.1, the finite amplifier DC gain affects the NTF characteristic and therefore decreases the DR. This can be observed in Fig. 3.7, where the variation of DR with the value of DC gain for all loop integrators is plotted for three designs. The DC gain of all opamps in the integrators is plotted on the $\mathrm{X}$-axis. No distinction is made between different integrators (according to the loop gain associated with each of them) because this effect changes the behavior of the entire $\Sigma \Delta$ loop filter [4]. The three curves are for fifth-order designs with 3-bit quantizers. One of the curves is for a singleloop operated at $\mathrm{OSR}=32$ and the other two are for 3-loop cascades operated at OSRs of 16 and 32 respectively. The graph is drawn from a "design" point of view, with the three lines stopping when the DC gain guarantees a DR of $98 \mathrm{~dB}$ for the respective architecture. It can be seen that a DC gain of $24 \mathrm{~dB}$ is enough for the single-loop solution (in line with what is recommended in design text books [4]), but $60 \mathrm{~dB}$ are needed for the cascade operated at OSR $=16$. The cascade designed for an increased OSR $(\mathrm{OSR}=32)$ needs an even higher DC gain in the amplifiers, larger than $72 \mathrm{~dB}$ as seen on the graph.

Traditionally, is said that gain linearity is not important in SC integrators. This is true as long as the DC gain of the amplifier is large enough to keep a low voltage at its inputs after the integration phase or as long as the gain, even if small, does not vary with the output voltage. Both these assumptions tend to be false in high-speed CMOS processes, where the output impedance of MOS transistors is small and shows a high dependence on the drain-source voltage. By replacing the $A_{D C}$ in Eq. 3.9 with an expression dependent on $V_{\text {out }}$, the importance of the gain nonlinearity can be estimated. From SPICE 


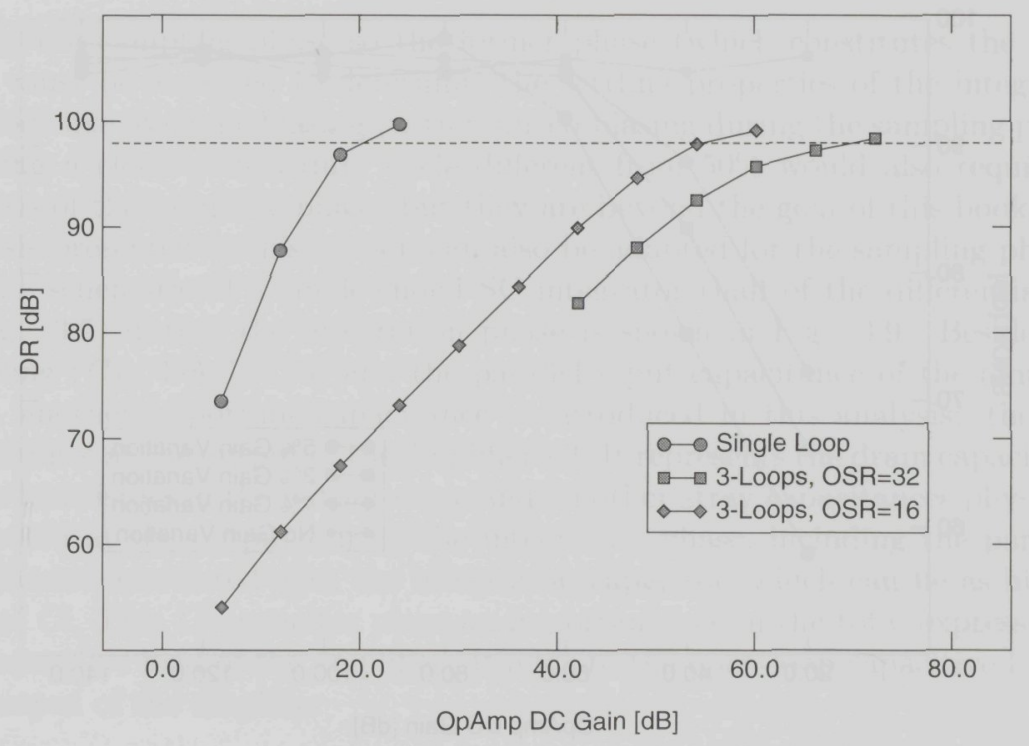

Figure 3.7: DR variation with opamp DC gain for both multi-loop and singleloop $A D C s$

simulation it has been found that, for a single-stage amplifier in $0.18 \mu m$ CMOS, the gain variation is as high as $\Delta A=6 \%$ for a peak differential output swing $\max \left(\left|V_{\text {out }}\right|\right)=2 \mathrm{~V}$, only caused by changes in the output impedance, and hence the variation is approximately linear with the output voltage:

$$
A_{D C}\left(V_{\text {out }}\right)=A_{D C}(0)\left(1-\frac{\left|V_{\text {out }}\right|}{\max \left(\left|V_{\text {out }}\right|\right)} \Delta A\right)
$$

A set of simulations have been run assuming $\Delta A$ values from 0 to $5 \%$ for a fourth-order, 5-bit $\Sigma \Delta$ ADC with a target peak SNDR of $96 \mathrm{~dB}$. The capacitors of the first integrator have been calculated from the noise considerations and for each $\Delta A$ value a search has been performed for the minimal $A_{D C}(0)$ value that reduces the harmonic distortion sufficiently to guarantee the target SNDR. The results are plotted in Fig. 3.8, with $A_{D C}(0)$ on the X-axis and the attained peak SNDR on the Y-axis. The simulations show that, for large $A_{D C}(0)$, large $\Delta A$ (nonlinearity) can be tolerated but the performance decreases rapidly for a combination of low $A_{D C}(0)$ and high $\Delta A$. This can be the case in fast-settling amplifiers which have to accommodate a large output swing in low-voltage, high-speed CMOS processes. 


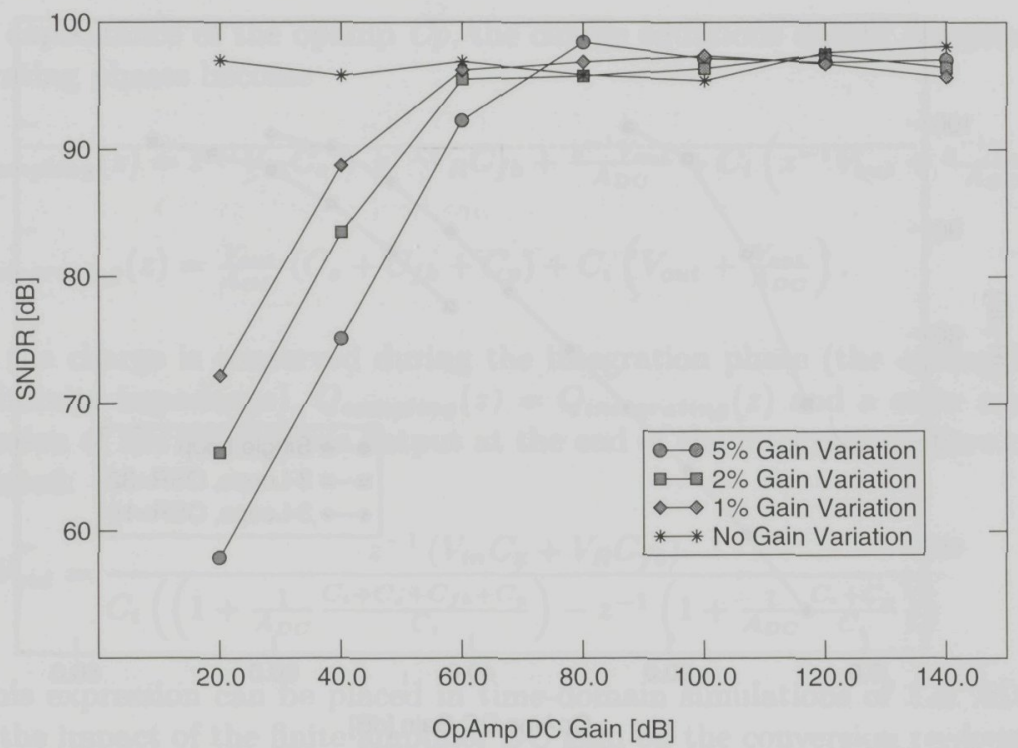

Figure 3.8: SNDR variation through signal-dependent opamp gain

\section{Finite Amplifier Bandwidth and Slew-Rate}

Switched-capacitor circuits count on fast charge transfer from the sampling and DAC capacitors to the integrating capacitor, and so far, in a first-order approximation, this transfer has been considered to be much faster than the sampling speed. This is only true if the unity-gain frequency of the amplifier (loaded with all the switching capacitors) is extremely large compared to the sampling frequency, which is almost never the case. In SC circuits, the limited amplifier bandwidth introduces settling errors at each integration and sampling phase, and hence it introduces harmonic distortion in the signal processing since the settling errors are signal dependent. In practical circuits the amplifier bandwidth is minimized to reduce power consumption, and the lower limit is set by the total harmonic distortion which can be introduced by the integrator. For a complete converter, the distortion introduced by each integrator has to be analyzed independently and each integrator's bandwidth has to be designed according to the associated loop gain (see Chapter 2). For example, if the first integrator in a $\Sigma \Delta \mathrm{ADC}$ has to settle to $-96 \mathrm{~dB}$ (16 bits) and its gain at the end of the signal bandwidth is $30 \mathrm{~dB}$, the second integrator only needs a THD of $-66 \mathrm{~dB}$ to contribute as much to the total THD as the first integrator.

Settling errors are introduced both during sampling and integrating phases. In designs with static biasing and clocked with $50 \%$ duty cycle, the capacitive load of the amplifier during the integration phase is always larger than the load 
during the sampling phase so the former phase (which constitutes the worst case) must be analyzed to determine the settling properties of the integrator. Methods like dynamic biasing (with reduced biasing during the sampling phase) or using a clock with a duty cycle different from $50 \%$ would also require an analysis of the sampling phase, but they are beyond the goal of this book. The analysis presented in this section can also be adapted for the sampling phase.

The schematic of a single-ended SC integrator (half of the differential one in Fig. 3.5) during the integration phase is shown in Fig. 3.9. Besides the sampling $(C s)$, DAC $(C f b)$ and the parallel input capacitance of the amplifier $(C p)$, another important capacitance is introduced in this analysis: the load capacitance at the output of the amplifier $\mathrm{Cl}$. It represents the drain capacitance of the transistors in the output stage and all other stray capacitances physically connected at the output during the integration phase, including the parasitic capacitance associated with the integration capacitor, which can be as high as $50 \%$ of $\mathrm{Ci}$. This $\mathrm{Cl}$ capacitor plays an important role in the total expression of the capacitive load of the amplifier. It adds to the open-loop capacitive load at the output of the amplifier

$$
C_{o l}=C_{l}+\frac{C_{i}\left(C_{s}+C_{f b}+C_{p}\right)}{C_{i}+C_{s}+C_{f b}+C_{p}}
$$

and, with a loop transfer factor of

$$
f_{\text {int }}=\frac{C_{i}}{C_{s}+C_{f b}+C_{p}+C_{i}}
$$

the closed-loop capacitive load of the amplifier is boosted by the presence of $C l$ :

$$
C_{c l}=\frac{C_{o l}}{f_{\text {int }}}=C_{s}+C_{f b}+C_{p}+C_{l}+\frac{C_{l}\left(C_{s}+C_{f b}+C_{p}\right)}{C_{i}} .
$$

The boost in capacitive load is larger than the ratio of $C_{l} / C_{i}$ and it explains why 2-layer poly-poly capacitors, which have a $C_{l} / C_{i}=0.25$, are more convenient

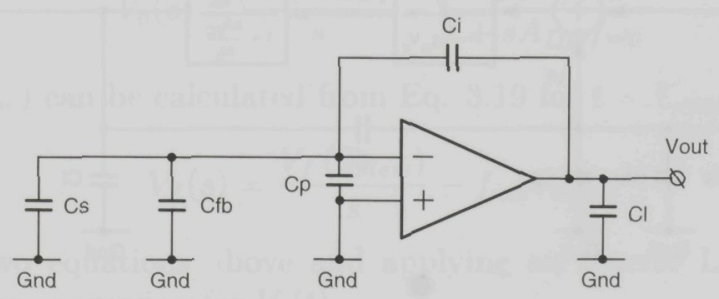

Figure 3.9: SC integrator during integration phase 
than 2-layer metal-metal capacitors, which have $C_{l} / C_{i}=0.50$ in typical a CMOS process.

The amplifier's behavior must be modeled next, to analyze the evolution of the output voltage during the integration phase. The opamp model is placed in the feedback loop, as shown in Fig. 3.10. It contains both a slewing component and a settling one [5]. The slewing component accounts for the limited current capability of an amplifier which is not dynamically biased, denoted in the model by the limit value of $I_{0}$ (which for a differential input pair is the tail current), and for the finite transconductance $g_{m}$ of the input stage. The slewing component accounts for the DC gain $A_{D C}$ and the finite unity-gain bandwidth, $\omega_{0}$.

With this model, the evolution of all voltages must be followed from the moment when the integrating phase starts. At first, there is no response from the opamp (which reacts only with finite speed to any stimulus), so a fast, passive charge re-distribution takes place and the feedback and output voltages become [6]

$$
\begin{aligned}
& V_{f}^{+}=\left(1+\frac{C_{l}}{C_{i}}\right) \frac{-C_{s} V_{i n}-C_{f b} V_{R} D A C+\left(C_{s}+C_{f b}+C_{p}+\frac{C_{i} C_{l}}{C_{i}+C_{l}}\right) V_{f}^{-}}{C_{o l}} \\
& V_{o}^{+}=V_{o}^{-}-\frac{C_{s} V_{i n}+C_{f b} V_{R} D A C+\left(C_{s}+C_{f b}\right) V_{f}^{-}}{C_{o l}}
\end{aligned}
$$

where $V_{f}^{+}$and $V_{o}^{+}$are the feedback and output voltages after the passive charge re-distribution respectively, and $V_{f}^{-}$and $V_{o}^{-}$are the corresponding voltages before the integration switches are closed. The equations show how the input charges $C_{s} V_{i n}$ and $C_{f b} V_{R} D A C$ (DAC is a $+/-1$ symbol showing the previous decision of the $\Sigma \Delta$ loop quantizer) affect the state of the two voltages of interest. The feedback voltage $V_{f}^{-}$is given by the output $V_{o}^{-}$and the DC gain

$$
V_{f}^{-}=-\frac{V_{o}^{-}}{A_{D C}} .
$$

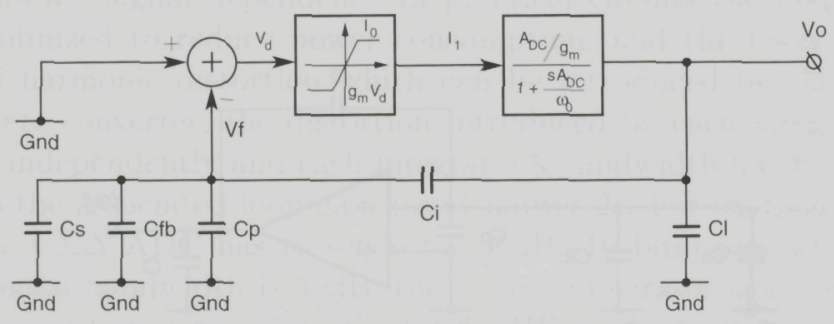

Figure 3.10: Functional modeling of real amplifier in a SC integrator during integration phase 
After the charge re-distribution, if

$$
\left|V_{f}^{+}\right|>\left|\frac{I_{0}}{g_{m}}\right|
$$

the amplifier slews at $I_{0}$, i.e.

$$
\begin{aligned}
& I_{1}(s)=\frac{I_{0}}{s} \operatorname{sgn}\left(-V_{f}^{+}\right) \\
& V_{o}(s)=\frac{V_{o}^{+}}{s}+\frac{A_{D C} / g_{m}}{1+s A_{D C} / \omega_{0}} I_{1}(s) .
\end{aligned}
$$

By an inverse Laplace transform, the expression of $V_{o}(t)$ is calculated

$$
V_{o}(t)=V_{o}^{+}+\frac{\operatorname{sgn}\left(-V_{f}^{+}\right) I_{0} A_{D C}}{g_{m}}\left(1-\exp \left(-\frac{\omega_{0}}{A_{D C}} t\right)\right)
$$

and by approximating $\exp (-x) \approx 1-x$ (holding for very small values of $x$ )

$$
V_{o}(t) \approx V_{o}^{+}+\frac{\operatorname{sgn}\left(-V_{f}^{+}\right) I_{0} \omega_{0}}{g_{m}} t
$$

The end of the slewing is reached when the feedback voltage evolving according to

$$
\left|V_{f}(t)\right|=\left|V_{f}^{+}+f_{\text {int }} V_{o}(t)\right|
$$

becomes equal with $\left|\frac{I_{0}}{g_{m}}\right|$, which gives a slewing time of

$$
T_{\text {slew }}=\left(V_{f}^{+}-\frac{I_{0}}{g_{m}}\right) \frac{C_{o l}}{I_{0} f_{\text {int }}}=\left(V_{f}^{+}-\frac{I_{0}}{g_{m}}\right) \frac{C_{c l}}{I_{0}}
$$

where $C_{o l}, f_{\text {int }}$ and $C_{c l}$ have been defined in Eqs. $3.11,3.12$ and 3.13 respectively.

After $T_{\text {slew }}$ the amplifier starts to settle and the expression of the output voltage can be calculated from

$$
V_{o}(s)=\frac{V_{o}\left(T_{\text {slew }}\right)}{s}-\frac{A_{D C} V_{f}(s)}{1+s A_{D C} / \omega_{0}}
$$

where $V_{o}\left(T_{\text {slew }}\right)$ can be calculated from Eq. 3.19 for $t=T_{\text {slew }}$ and

$$
V_{f}(s)=\frac{V_{f}\left(T_{\text {slew }}\right)}{s}-f_{\text {int }} V_{o}(s)
$$

Solving the two equations above and applying an inverse Laplace transform yields a settling expression for $V_{o}(t)$

$$
V_{o}(t)=V_{o}\left(T_{\text {slew }}\right)+\frac{A_{D C} V_{f}\left(T_{\text {slew }}\right)}{1+A_{D C} f_{\text {int }}}\left(1-\exp \left(-\omega_{0} \frac{1+A_{D C} f_{\text {int }}}{A_{D C}} t\right)\right) .
$$


This equation shows the exponential settling undergone by the output, asymptotically evolving toward its ideal value. Using this expression for the output voltage in a time-domain simulator, the effects of the finite $\omega_{0}$ of the amplifier can be observed as distortion introduced by the $\Sigma \Delta \mathrm{ADC}$ and a minimal bandwidth value can be calculated by assuming a single-pole system. This will be used in a subsequent section to estimate the power consumption of an integrator required to settle to a certain number of bits.

\section{Non-ideal MOS Switch}

In CMOS SC integrators, the switches used to control the charge transfer are MOS transistors, which approximate an ideal switch in the sense that there is no DC connection between the ON/OFF control (the gate of the MOS transistor) and the signal path (the drain and source nodes). This is an important property of MOS switches and it guarantees that the control and signal paths are separated. Unfortunately, this is only true at or near DC, in other words for slow-varying control clocks and signals, which is definitely not the case in oversampling converters.

A SC integrator complete with NMOS switches is shown in Fig. 3.11. The input switch $s w 1$ is represented as an ideal MOS switch plus the most important parasitics which limit its performance, the gate-source and gate-drain capacitors ( $C g s$ and $C g d$ respectively) and the ON-resistance (Ron). The two gate capacitances open a finite-impedance path between the control node and the two signal nodes, the drain and the source. This high-frequency connection introduces charge into the signal nodes (clock feedthrough) on the rising and falling edges of the control clock and, if these edges are not symmetrical, a voltage offset is integrated [7].

The ON-resistance adds another pole in the behavior of the integrator, both during the sampling and the integrating phase. During the sampling phase, the

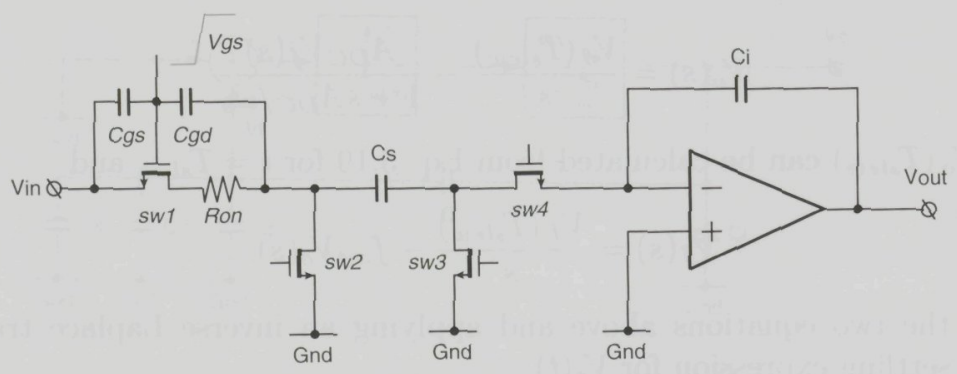

Figure 3.11: SC integrator with NMOS switches 
voltage sampled on $C s$ is

$$
V_{\text {sampled }}=V_{\text {in }}\left(1-\exp \left(-\frac{t}{2 C_{s} R_{o n}}\right)\right)
$$

and during integrating phase the output voltage evolves as

$$
V_{\text {out }}=V_{\text {out }}(0)+\frac{C_{s}}{C_{i}} V_{\text {sampled }}\left(1-\exp \left(-\frac{t}{2 C_{s} R_{\text {on }}}\right)\right) .
$$

Two conclusions become apparent: both sampling and integration are subject to exponential settling dictated by the Cs and Ron, and the errors during the two phases add (assuming $\exp \left(-\frac{t}{2 C_{s} R_{o n}}\right)<<1$ ). This introduces an error in the integration coefficient compared to the ideal $C_{s} / C_{i} . R_{o n}$ must be reduced by design to avoid significant degradation of the NTF shape. In practice, $R_{\text {on }}$ of the floating sampling switch $s w 1$ can also be non-linearly dependent on the input signal, making the settling error signal dependent as well and therefore producing harmonic distortion. Reducing $R_{\text {on }}$ also helps in this case.

The reduction of Ron is not favorable for another parasitic effect: the charge injection in the MOS channel. From basic MOS transistor theory [10], the charge that is released from the channel into the source and drain when the MOS switch is turned off is

$$
Q_{i n j}=\frac{L^{2}}{\mu R_{o n}}
$$

and it depends on the switch length $L$, the on-resistance $R_{\text {on }}$ and also on the type of MOS switch through the mobility of carriers in the channel, $\mu$. The faster the switch ( $L$ smaller, $R_{\text {on }}$ smaller proportionally at constant gate overdrive $\left.V_{g s}-V_{T}\right)$, the larger the charge injected. The charge injected by $s w 1$ is also non-linearly signal dependent and can introduce harmonic distortion.

The effects of both clock feedthrough and charge injection can be reduced with orders of magnitude by adding dummy switches [4] and employing differential circuits [9]. In these circuits, all the common-mode effects are attenuated by their CMRR, which can have values larger than $60 \mathrm{~dB}$ even in low-voltage, high-speed designs. Therefore, only the part of the error charges which is signal dependent remains an important issue. Methods to reduce these non-linear errors will be explained in the next sub-section.

\subsubsection{Clocking Signals}

\section{Clock Phases}

By looking at Fig. 3.11, one can see why it is important that not all switches are closed simultaneously. Suppose that during the sampling phase, when sw1 and sw2 are closed, sw4 is also closed or weakly closed (small $V_{g s}-V_{T}$, therefore 


\section{Discrete-Time Circuit Design}

large $\left.R_{o n}\right)$. In this case there is charge leaking from the integration capacitor, amounting to approximately

$$
\Delta Q_{C i}=-\frac{V_{o}}{A_{D C} R_{\text {on }}^{\text {sw }}} t_{\text {overlap }}
$$

Since the charge lost is proportional to the output voltage, which in a $\Sigma \Delta$ ADC contains the input signal spectrum, this effect is expected to put harmonic distortion in the $\mathrm{A} / \mathrm{D}$ conversion.

A simple yet efficient way to deal with this problem is to make sure that the active periods of the sampling and integration clock signals do not overlap. This can be achieved with a digital latch as the one shown in Fig. 3.12. The Master CLK is the reference clock (generated on-chip or supplied from off-chip) and is used to drive the RS NOR-latch. The outputs are delayed compared to the reference clock and they do not overlap if the delay through the NOR gates is larger than the edge duration. If this is not the case, additional delay can be introduced in the latch loop by using inverters.
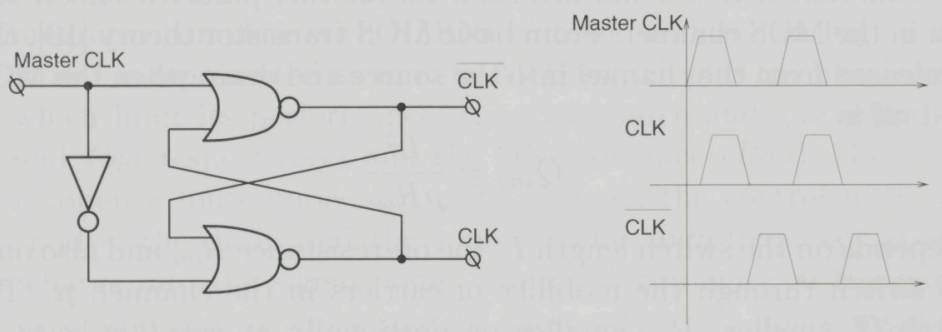

Figure 3.12: Latch configuration used to generate non-overlapping clocks

\section{Reduction of Non-linear Switching Errors}

Correct timing of the four clock signals does not only prevent charge sharing between the sampling and integrating phases, it can also reduce the non-linear charge injection which is generated by the switches with floating drain and source, i.e. sw1 and sw4 in Fig. 3.11, when they are switched off. Assuming that, at the end of the sampling phase, the switch sw3 is turned off before the switch sw1 (and both of them switch off before any of sw2 and sw4 switch on), then the charge injected by sw1 will all be taken to the source of the NMOS switch which is connected to the low-impedance signal source Vin. In this way, the amount of sw1-injected charge actually sampled on $C s$ is minimized. Of course, the charge injected by $s w 3$ is still taken by $C s$ but it is not signal 
dependent since the source of sw3 is connected to a fixed potential, Gnd. A similar logic can be applied to the sw2-sw4 switch pair for the integrating phase.

The circuit used to generate these delayed clock phases is derived from the one used for non-overlapping clocks since the sampling and integration clocks should still not overlap. By adding an even number of inverters in the latch loop, as shown in Fig. 3.13, additional delays can be added in increments of two inverter delays, both between opposite-phase clocks (total sampling integration periods) and between different phases of the same clock: sampling for sw3, delayed sampling for sw1, integration for sw 4 and delayed integration for $s w 2$.

Besides correct phasing of the clocks, there is another technique aimed at reducing the non-linear signal-dependent component of the charge injected especially by the input switch, sw1. From Eq. 3.27 it can be seen that the charge injected by a MOS switch is inversely proportional to the ON-resistance, $R_{\text {on }}$. For a MOS switch in the linear region $\left(V_{d s}<<V_{g s}-V_{T}\right.$ for NMOS) $R_{o n}$ depends linearly (in a first approximation) on both $V_{g s}-V_{T}$ and $V_{d s}$, so any charge injected in the sampling capacitor $C s$ should not introduce distortion. However, in low-voltage designs, most of the time the condition $V_{d s}<<V_{g s}-V_{T}$ is barely satisfied and then the NMOS switch has an $R_{\text {on }}$ which is quadratically or even exponentially dependent on $V_{g s}-V_{T}$. If the clock (the gate potential) is a digital signal switching between ground $(\mathrm{OFF})$ and $V_{d d}(\mathrm{ON})$, the difference $V_{g s}-V_{T}$ contains an input-signal-dependent component which will introduce non-linear components in the sampled signal. This effect can require drastic limitation of the input signal range in low-voltage designs.

Two ways can be envisioned to deal with this non-linear charge injection: boosting of the clock signal voltage to larger values to increase $V_{g s}-V_{T}$ for the whole Vin range and operating the switch in the linear region, or reduction of

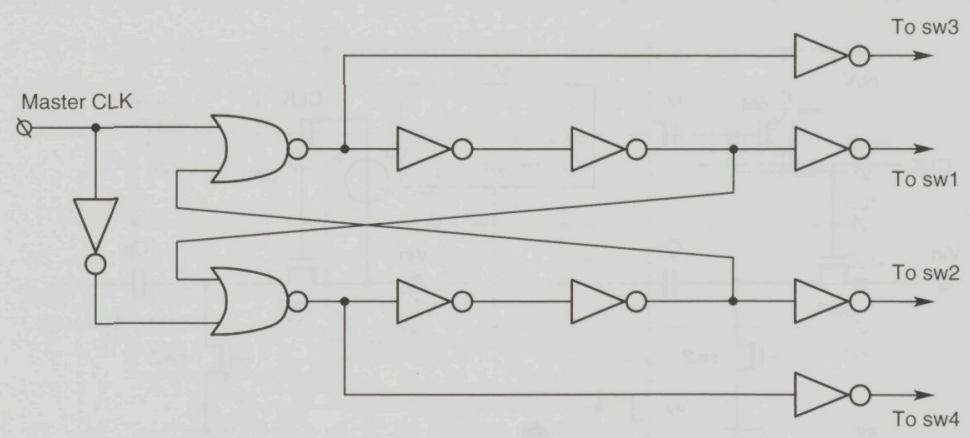

Figure 3.13: Latch configuration used to generate non-overlapping clocks and their delayed versions 


\section{Discrete-Time Circuit Design}

the signal dependency of the $V_{g s}-V_{T}$ expression and therewith reduction of the non-linear component of the charge injected.

The first approach is illustrated in Fig. 3.14 (left), where the CLK signal is a "digital" clock switching between ground and $2 V d d$. The quotation marks around "digital" emphasize that, most of the times, $V d d$ is the largest voltage available on-chip and therefore the $2 V d d$ clock signal $C L K$ has to be generated (on-chip) using charge pumps. Circuits to realize this doubling of the clock voltage have been reported in many books and papers [8] [6]. It is worth noting that, with this technique applied, the voltage across the gate oxide is $2 V d d$ during all of the sampling phase if the input signal is close to ground. Obviously, a CMOS technology which tolerates $2 \mathrm{Vdd}$ across the gate oxide has to be used. For example, it is possible to design a $1.8 \mathrm{~V}$ SC $\Sigma \Delta$ ADC in $0.5 \mu m$ CMOS since the technology tolerates a maximal $V_{g s}$ of $5 \mathrm{~V}$ and the doubled clock signal would reach $3.6 \mathrm{~V}$ (see the design example in Chapter 6 ). The same design in a $0.35 \mu \mathrm{m}$ CMOS would push the technology tolerance to its limits (nominal $V_{g s}$ for $0.35 \mu \mathrm{m}$ CMOS is $3.3 \mathrm{~V}$ ), and it would be impossible to carry it out in $0.18 \mu \mathrm{m}$ CMOS, which has a nominal $V_{g s}$ of $1.8 \mathrm{~V}$.

A technique that circumvents this disadvantage is shown in Fig. 3.14 (right). Instead of a fixed ON-voltage, the gate of the switch now has a variableamplitude $\mathrm{ON}$-voltage which follows the source voltage Vin. In the ON-state the gate potential is always $V b$ larger than the source potential, hence the $R_{o n}$ of the switch is signal-independent. Usually $V b$ is comparable to $V_{d d}$ in magnitude so, besides being signal-independent, $V_{g s}-V_{T}$ is also large and the switch is operated in saturation. Reliability is well improved with this technique. The gate oxide only takes a large voltage until the inversion layer is formed under the gate, then the voltage across the oxide drops to $V b$. Since this is less than $V_{d d}$, during most of the sampling period, the gate oxide is biased below the technology-tolerated voltage. One circuit which can be used to implement this "floating battery" technique in CMOS will be described in Chapter 7 . It
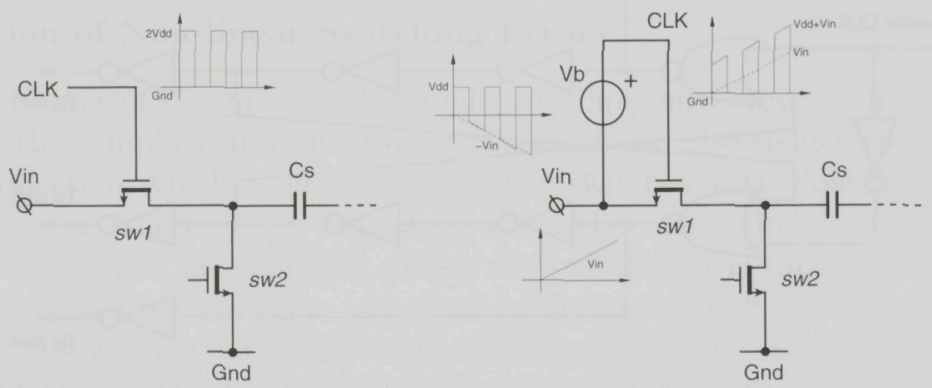

Figure 3.14: Two approaches to clock boosting 
has been successfully used in a SC integrator with 14 bits linearity designed in $0.18 \mu m$ CMOS with $V_{d d}=1.8 \mathrm{~V}$.

\subsubsection{Operational Amplifier Topologies}

Even if class-AB amplifiers show less static power consumption for a similar settling time than class- $\mathrm{A}$ amplifiers, they are not easy to implement in low-voltage applications. A typical class-AB amplifier requires two stages: a static-biased input stage and a class- $\mathrm{AB}$ output stage, which means more power consumption. Furthermore, a two-stage differential class-AB amplifier may need two common-mode feedback control circuits [8], which makes it more power hungry and/or more complex. Therefore, for high-performance low-voltage designs the class-A amplifiers are mostly used, and a few of these will be explained further on in this sub-section.

From the behavioral models described in the previous sub-section, the most important parameter of an opamp to be used in a SC integrator is its bandwidth. The amplifier loaded with all the capacitive paths has to settle to the required accuracy in the available time, usually half the clock period. Since large bandwidth (high switching speed) equates low-complexity designs (the best example is a digital inverter), Fig. 3.15 shows the fastest class-A amplifier, given a capacitive load and a supply current. It consists of only the transconductance stage $M 1$ and M2 and the active load M3 and M4. The rest of the transistors are biasing the circuit. The DC voltage gain of this amplifier is

$$
A_{D C}=\frac{g_{m_{M 1}}}{g_{d s_{M 1}}+g_{d s_{M 3}}}
$$

and has a maximal value of $40 \mathrm{~dB}$ in typical CMOS processes, which is mainly limited by the output impedance of the NMOS transistors. These transistors

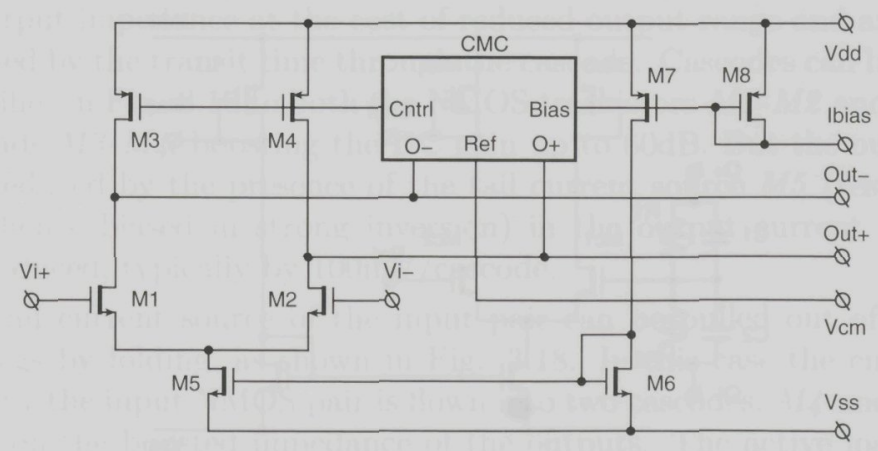

Figure 3.15: Single-stage, fast operational amplifier 


\section{Discrete-Time Circuit Design}

are usually designed with a large $g_{m_{M 1}} / I_{d_{M 1}}$ to push the unity-gain bandwidth (UGB) of the amplifier loaded with $C_{\text {ol }}$ (see sub-Section 3.1.2)

$$
U G B=\frac{g_{m_{M 1}}}{2 \pi C_{o l}}
$$

to the value imposed by settling requirements, with a minimal drain current $I_{d_{M 1}}$. A large $g_{m_{M 1}} / I_{d_{M 1}}$ means that $M 1$ is designed with a large $\mathrm{W} / \mathrm{L}$ to push it in moderate or weak inversion, hence the output impedance is reduced. Since the gate capacitance (or the product WL) has to be kept minimal (see effects of $C p$ in sub-Section 3.1.2), a small channel length is typically used, which, in high-speed CMOS, further reduces the output impedance.

The common-mode voltage at the input of the amplifier is controlled by the previous stage or by the common mode of the input signal for the first integrator. The common-mode voltage of the output, however, has to be controlled inside the amplifier. This is accomplished by the common-mode control (CMC) block, which implements a feedback loop to force the common-mode output voltage to be equal with the desired common-mode $V \mathrm{~cm}$ (present at the input Ref of the CMC block). A CT version of the CMC is shown in Fig. 3.16. It uses a differential pair $M c 1$ and $M c 2$ to compare the desired common-mode voltage at $R e f$ with the common-mode voltage of the two read-in amplifier outputs $O+$ and $O$-. The latter voltage is available on the resistive divider $R 1-R 2$. The feedback loop is closed through the Cntrl output which controls the gate potential of the active loads in Fig. 3.15. Because a feedback loop is closed through two amplifying stages, stability can be a concern if the load capacitors (mainly the $C_{g s}$ of $M 3$ and $M 4$ in Fig. 3.15) are large. The parallel-compensation capacitors $C 1$ and $C 2$ are used to avoid the extra pole at the gate of $M c 1$ within the CM loop.

The SC variant (Fig. 3.17) of the CMC does not raise a stability issue since

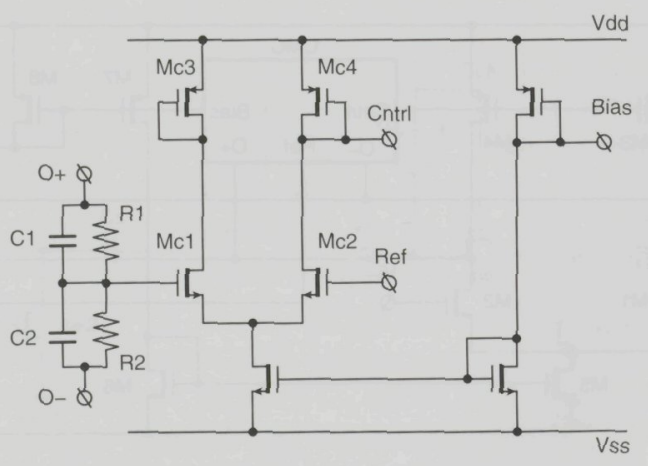

Figure 3.16: Continuous-time common-mode feedback control 


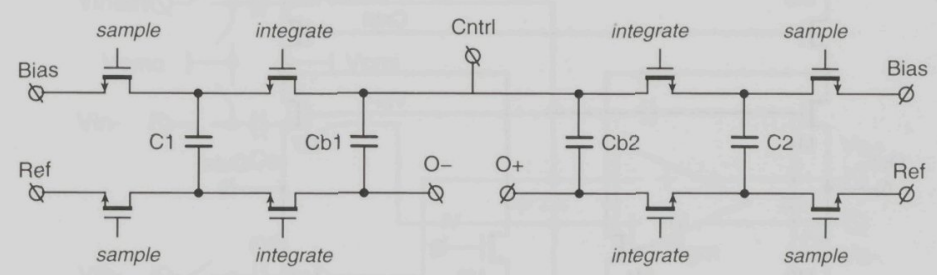

Figure 3.17: Switched-capacitor common-mode control

the common-mode feedback amplifier has only one stage: the transistors $M 3$ and $M 4$ in Fig. 3.15. This CMC uses two capacitors, $C 1$ and C2 in Fig. 3.17, to sample the difference between the Ref voltage and the Bias voltage, and two other capacitors to maintain this voltage difference between the gate of the active loads $M 3$ and $M_{4}$ (Fig. 3.15) and the outputs $O+$ and $O$-. The sampling and integrate signals are the corresponding clock phases used to operate the entire SC integrator. Apart from always being stable, the SC CMC also has the advantage of being able to work with a larger range of Ref voltages, as opposed to the CT version in Fig. 3.16, which can only operate with Ref larger than one saturation plus one gate-source voltage. The SC CMC can work with any Ref voltage if the right type of MOS switch is used (NMOS for low Ref, PMOS for high Ref, or transmission gates for any Ref voltage). A drawback of using a $\mathrm{SC}$ CMC is the additional switching load present at the output of the amplifier, which is important in fast-settling, low-distortion SC integrators.

If the DC gain has to be increased, as is the case in cascaded $\Sigma \Delta$ ADCs, where a low DC gain can decrease DR performance by altering the shape of NTF through lossy integration, cascodes must be employed. They offer a boost in the output impedance at the cost of reduced output range and an additional pole caused by the transit time through the cascode. Cascodes can be applied in the amplifier in Fig. 3.15 to both the NMOS transistors M1-M2 and the PMOS active loads $M 3-M 4$, boosting the DC gain up to $60 \mathrm{~dB}$. But the output range, already reduced by the presence of the tail current source M5 (responsible for CMRR, hence biased in strong inversion) in the output current legs will be further reduced, typically by $100 \mathrm{mV} /$ cascode.

The tail current source of the input pair can be pulled out of the output current legs by folding, as shown in Fig. 3.18. In this case the current signal supplied by the input NMOS pair is flown into two cascodes, $M 4$ and $M 7$, which repeat it on the boosted impedance of the outputs. The active loads M3 and M6 can also be cascoded, but in low-voltage designs it would be preferable to just design them with a large output impedance. The voltage gain of the 


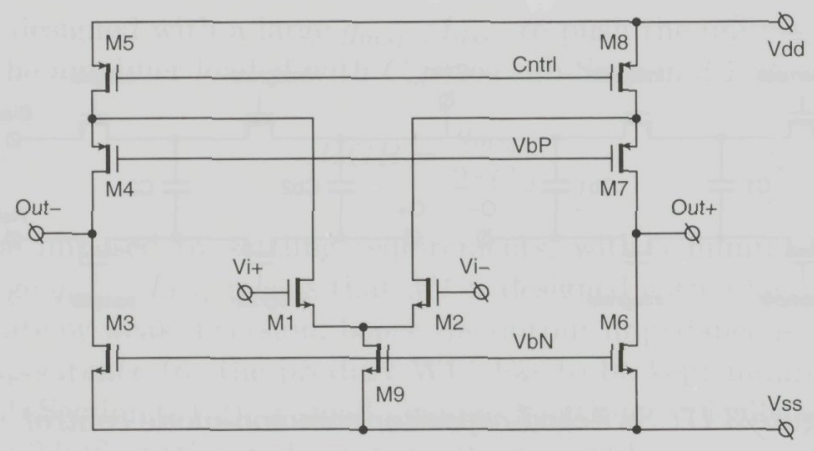

Figure 3.18: Folded-cascode single-stage amplifier

folded-cascode amplifier is

$$
A_{D C}=\frac{g_{m_{M 1}}}{g_{d s_{M 6}}+\frac{g_{d s_{M 8}}}{1+g_{m_{M 7}} / g_{d s_{M 7}}}} .
$$

Since this is also a one-stage design, stability is not an issue unless the UGB (given by an equation similar to Eq. 3.30) is comparable to the pole introduced by the transit time through the cascode transistors. The same CMC circuits can be used in both the folded-cascode amplifier and the simple single-stage one.

\subsubsection{Power Consumption Analysis}

The transconductance of the opamp inside a SC integrator is a good model of the power consumption of the integrator, especially if the amplifier is a onestage design. Starting from the noise budget available for the integrator, one can calculate the capacitors building the $\Sigma \Delta$ coefficients. With the capacitor values and the distortion requirements (translated in settling constraints), the $g_{m}$ of the opamp's input stage can be calculated. The power is evaluated by considering fully differential circuits are used, with independent paths for input and DAC signal integration, as shown in Fig. 3.19.

\section{Noise}

The noise budget allocated to each integrator (based on designer expertise or by $\mathrm{CAD})$ is consumed by two components: the switch noise $(k T / C$ noise $)$ and the opamp noise. Consider a one-stage amplifier with a large input transconductance $g_{m}$ : its noise is given by the differential input stage for a UGB inversely 


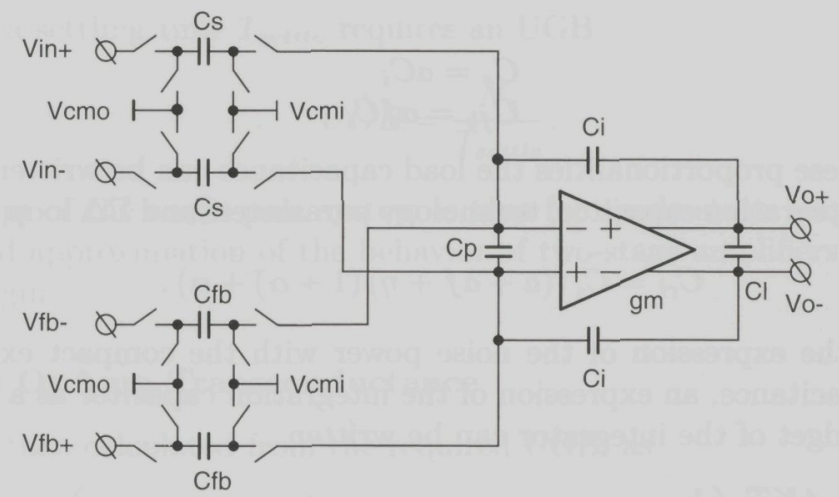

Figure 3.19: Differential SC integrator with DAC

proportional to the closed-loop load capacitance $C_{c l}$

$$
\begin{aligned}
& U G B=\frac{g_{m}}{2 \pi C_{c l}} \\
& P_{n_{a m p}}=\frac{2 k T \gamma}{g_{m}} U G B=\frac{k T \gamma}{\pi C_{c l}}
\end{aligned}
$$

with $\gamma$ the noise enhancement factor for short-channel transistors [6] (applicable for opamp input devices). The total noise power (wide-band) at the input of the SC integrator is

$$
P_{n}=4 K T\left(\frac{1}{C_{s}}\left(1+\frac{C_{f b}}{C_{s}}\right)+\frac{\gamma}{4 \pi C_{c l}}\right)
$$

with

$$
C_{c l}=C_{s}+C_{p}+C_{f b}+C_{l}+\frac{C_{l}\left(C_{s}+C_{p}+C_{f b}\right)}{C_{i}}
$$

(as explained in one of the previous sub-sections), where $C_{l}$ is a fraction of the integration capacitor (parasitic capacitance of $C_{i}$ ) connected at the opamp's output

$$
C_{l}=\alpha C_{i}
$$

where $\alpha$ is a technology-dependent number expressed as a percentage. $C_{p}$ is also proportional to $C_{i}$ since, for a given $V_{g s}-V_{T}$ and UGB, the transistor gate area is relatively proportional to the capacitive load:

$$
C_{p}=\eta C_{i} .
$$

The $C_{s}$ and $C_{f b}$ capacitors are related to the integration capacitor through the $\Sigma \Delta$ loop coefficients supplied by the architecture design (see Chapter 2, Fig. 


\section{Discrete-Time Circuit Design}

2.9)

$$
\begin{aligned}
& C_{s}=a C_{i} \\
& C_{f b}=a f C_{i}
\end{aligned}
$$

and using these proportionalities the load capacitance can be written as a function of the integration capacitor, technology parameters and $\Sigma \Delta$ loop coefficients

$$
C_{c l}=C_{i}((a+a f+\eta)(1+\alpha)+\alpha) .
$$

Combining the expression of the noise power with the compact expression of the load capacitance, an expression of the integration capacitor as a function of the noise budget of the integrator can be written

$$
C_{i}=\frac{4 K T}{P_{n}}\left(\frac{1}{a}(1+f)+\frac{\gamma}{4 \pi((a+a f+\eta)(1+\alpha)+\alpha)}\right) .
$$

The first part of the noise power in Eq. (3.33) is the noise of the switch onresistance and the second part is the noise of the $g_{m}$ of the opamp's input pair. As part of the total noise budget (allocated for the entire converter), the noise power inside the signal bandwidth is referred at the input of the $\Sigma \Delta$ converter as

$$
P_{i}=\left(P_{n} \frac{1}{\prod_{j=1}^{i-1}\left(\frac{C_{s j}}{C_{i j}}\right)} \frac{\pi^{2(i-1)}}{(2 i-1) O S R^{2 i-1}}\right)_{i}
$$

with $i$ the order of the integrator in the $\Sigma \Delta$ converter. For example, the noise power contributed by the first integrator inside the signal bandwidth is

$$
P_{1}=\frac{1}{O S R} P_{n_{1}}
$$

if $P_{n_{1}}$ is calculated based on Eq. 3.33. Calculating the wide-band noise for each integrator and then referring it to the input of the $\Sigma \Delta$ loop through Eq. 3.40 is a convenient, unified way of calculating the contribution of each integrator to the total noise budget.

\section{Distortion}

Starting from a specification of harmonic distortion $\left|H D_{3}\right|$ (only the third one since differential circuits are used), the linearity of the integrator can be expressed in bits as [9]

$$
B \approx \frac{\left|H D_{3}\right|[d B c]}{6}
$$

The number of bits B is then used to calculate the number of time constants required by a single-pole system to settle to $\mathrm{B}$ bits [9]

$$
N_{\tau}=\operatorname{Bln}(2)
$$


which, for a settling time $T_{\text {settle }}$ requires an UGB

$$
U G B=\frac{N_{\tau}}{T_{\text {settle }}} .
$$

The concept of the single-pole system is true for single-stage amplifiers and is also a good approximation of the behavior of two-stage amplifiers with enough phase margin.

\section{Required OpAmp Transconductance}

The $g_{m}$ is then calculated from the required UGB, as

$$
g_{m}=2 \pi C_{c l} U G B=2 \pi C_{c l} \frac{N_{\tau}}{T_{\text {settle }}}
$$

For a slewing followed by settling model [6] as has been explained in sub-Section 3.1 .2 , the time available for settling is

$$
T_{\text {settle }}=\frac{T_{C L K}}{2}-T_{\text {slew }}
$$

with $T_{\text {slew }}$ calculated from Eq. 3.21. Hence, the expression of $g_{m}$ results in

$$
g_{m}=\frac{2 \pi C_{c l}\left(N_{\tau}-1\right)}{\frac{T_{C L K}}{2}-V_{f}^{+} \frac{C_{c l}}{I_{0}}}
$$

where $I_{0}$ is the biasing current (tail current) of the opamp MOS input pair and $V_{f}^{+}$is the voltage at the input of the opamp after passive charge re-distribution, explained with Eq. 3.14.

If MOS transistors operated in weak inversion are used in the input stage of the opamp, $g_{m}=10 I_{0}$, and a compact expression for $g_{m}$ is obtained

$$
g_{m}=\frac{4 \pi C_{c l}}{T_{C L K}}\left(\left(N_{\tau}-1\right)+10 V_{f}^{+}\right) .
$$

\subsection{Switched-Capacitor Amplifiers}

The feedforward coefficients in a SC loop filter can only be implemented with switched capacitors if they connect to the input of a SC integrator containing a virtual ground node where the charge can be transferred. However, feedforward coefficients coupling the output of each integrator with the quantizer input can be used to build a stable $\Sigma \Delta$ topology [11]. In such topologies it is preferable (especially at low-OSR and when high distortion performance is targeted) to 


\section{Discrete-Time Circuit Design}

perform the summation of the coefficient-weighted integrator outputs with a summing amplifier which supplies the signal to the comparator input. The main challenge in designing this amplifier is to add different-weighted signals without introducing an additional delay in the loop but still in a SC manner.

The summing amplifier in Fig. 3.20 is drawn with two input paths, reading Vin1 and Vin2 on the weight capacitors Cw1 and Cw2 during the integration phase denoted by the switching clock $i$ in the figure. This timing adds the $C w$ capacitors as extra capacitive loads to the corresponding amplifiers during the integration phase but performs the summation of all charges passed through the $C w$ capacitors on the capacitors $C a$ without delay. In this way, the sum of the input signals is available at the output of the amplifier at the end of the integration phase, ready to be used by the quantizer to make a decision at the beginning of the sampling phase.

During the sampling phase the summing capacitors $\mathrm{Ca}$ are reset by two switches so the circuit works as an amplifier and not as an integrator. At the same time the capacitors $C w$ are connected to fixed potentials V11, V12 and V21, V22 respectively. These potentials are designed to place common-mode voltages on the weight capacitors during the sampling phase, which are then used to keep a convenient common-mode voltage at the input of the opamp during the integration phase, even for different common-mode voltages of Vin1 and Vin2.

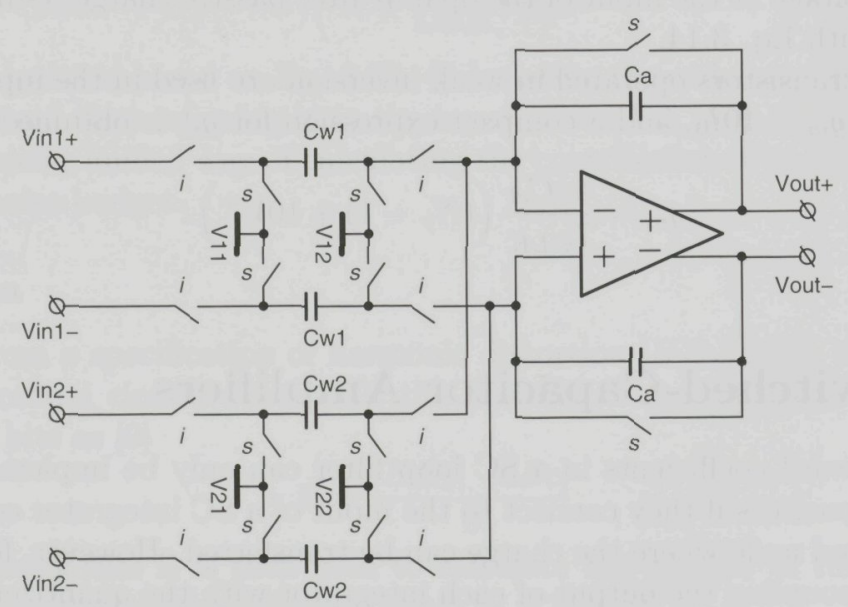

Figure 3.20: SC amplifier with zero-delay 


\subsection{Quantizer}

\subsubsection{The Dynamic Comparator}

The quantizer used in SC $\Sigma \Delta$ ADCs is usually built with one or multiple dynamic comparators based on a regenerative latch, like the one in Fig. 3.21. It uses an NMOS input pair $M 1-M 2$ as a transconductance-stage to generate a differential current on the M5-M6 PMOS latch and to switch it according to the input signal. The timing can be controlled by only one clock signal, CLK. With $C L K$ logic-Low, the input devices are switched off but still connected to the input signal so their gate capacitors are charged by the input voltage. At the same time, the switches $M 7$ and $M 8$ are turned on to reset the latch $M 5-M 6$ by pre-charging the outputs of the latch, thus reducing comparator hysteresis. This also connects the inputs of the RS digital NAND latch to $V d d$ (logic-High) forcing the RS latch to switch to the "memorize" state, keeping the previous decision stable at the digital output Out. When CLK is turned logic-High, the switches $M 7-M 8$ are turned on, connecting the input transistors to the PMOS latch. The latch is initially in a metastable condition and the differential current forced by $M 1$ and $M 2$ is forcing it to latch according to the sign of the input voltage.

The main advantages of this design are its reduced hysteresis and the completely dynamic operation showing no static power consumption. There are many variations of this design. Its gain can be increased by replacing the PMOS latch with a fully-complimentary latch made from two inverters. In low-voltage designs, there may be no tail current source [6] to save one saturation voltage at the price of uncontrolled supply-current spikes. In high-speed designs, folding

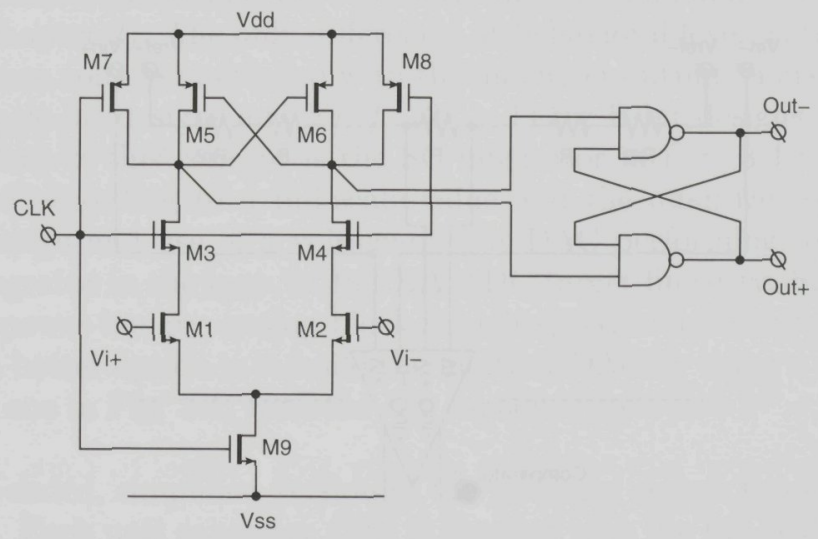

Figure 3.21: Dynamic Comparator 
of the current generated by M1-M2 may be employed to take advantage of the higher speed of an NMOS latch [9].

Relative to the integration clock phases, the comparator should be controlled by the sampling clock so that it makes a decision at the beginning of this phase. Its decision is needed for the DAC capacitors during the same phase, so slow comparators may charge the DACs incompletely, introducing signal-dependent charge effects, and hence distortion. Meta-stability may be a concern in highspeed processes where high gains are difficult to achieve, but usually the presence of the RS latch after the comparator relaxes the meta-stability issue significantly.

\subsubsection{Multibit Quantizers}

Multibit quantizer and DACs are implemented in $\Sigma \Delta$ ADCs to reduce in-band quantization noise power and to improve loop stability [9], especially when only a low OSR can be used and large DR is targeted. While a single-bit quantizer has a simple structure (like the one in Fig. 3.21), a B-bit quantizer employs $2^{B}-1$ that quantizers compare the input signal with reference voltages supplied by a resistive ladder biased by the full-scale reference voltage Vref, as shown in Fig. 3.22. In this case, two differential input stages have to be used inside the comparator to be able to compare only differences of voltages to reduce sensitivity to common-mode switching cross-talks and common-mode voltage variations of the input signal (an example is shown in Chapter 7). Hence, the comparator actually becomes a differential-difference comparator and care must be taken that, at full-swing signals, the difference of the reference and input signals is still performed with 1-bit accuracy. This implies the use of input stages with small $g_{m}$ or the use of large biasing currents. A compromise

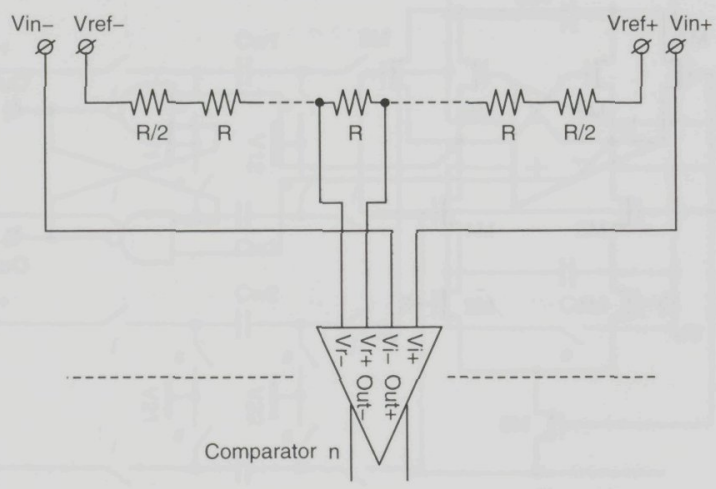

Figure 3.22: Multibit quantizer with resistive ladder 
has to be made during the design phase to balance the power consumption with the significant offset increase introduced by a small $g_{m}$. From $\Sigma \Delta$ ADC theory [4], the offset of the comparator only needs to be lower than the step of the reference voltages

$$
V_{\text {step }}=\frac{2\left(V_{r e f+}-V_{r e f-}\right)}{2^{B}-1} .
$$

The presence of the resistive ladder which introduces a variable, non-zero impedance at the input of every comparator in the quantizer also increases the sensitivity to comparator kick-back. At decision time, the switching of the latch inside the quantizer injects a charge in the gate-drain capacitances of the input MOS devices, a charge that has to be drained by the circuit driving the gate. On the reference inputs, the resistors in the ladder are driving the gate and are low-pass filtering the kick-back charge. This introduces switching dependence of the reference levels supplied to the comparator. Furthermore, the impedance present at the input of the comparator depends on the order of the comparator, with the lowest impedance at the ends of the ladder. This level-dependent kick-back introduces non-linear distortion in the $\Sigma \Delta$ ADC output and is the limiting effect for the maximal value of the resistors in the reference ladder, $R$. Other methods to reduce kick-back can also be applied before minimizing $R$, like designing 2-stage comparators whose pre-amplifier is a statically biased differential pair and judiciously placing large-valued resistors in the circuit to improve the backwards filtering of fast charge glitches.

\subsection{Multibit, SC Digital-to-Analog Converters}

In the design flow of $\Sigma \Delta$ ADCs, the loop coefficients are calculated based on behavioral simulations in similar ways for both single-bit and multibit-quantization loops (see Chapter 2). The only difference, at behavioral level, is the amplitude of the reference voltage multiplied with the quantizer output. This amplitude is Vref for single-bit designs and Vref/ $\left(2^{B}-1\right)$ for B-bit designs. The circuitlevel equivalent of this concept is the SC integrator in Fig. 3.4 where the $V R$ is modulated according to a full-scale value and the quantizer output. This needs the design and use of a voltage-output DAC performing, in the case of the first integrator in the loop, at full $\Sigma \Delta$ ADC target linearity. Such a DAC is a sensitive, power-hungry analog block if high speed and linearity are needed. Therefore, a better option is to build the multibit DAC by using a circuit topology like the one in Fig. 3.5, with the $C f b$ capacitor split into $2^{B}-1$ equal-sized capacitors.

A single-ended, simplified version of the resulting circuit topology is drawn in Fig. 3.23. Each unit capacitor $C f b u$ is charged with the full-scale DAC reference voltage, the sign depending on each comparator's decision $D A C 1$ to $D A C n$ ( $D A C 1$ to $D A C n$ take values $+/-1, \mathrm{n}=2^{B}-1$, DAC reference voltage assumed 


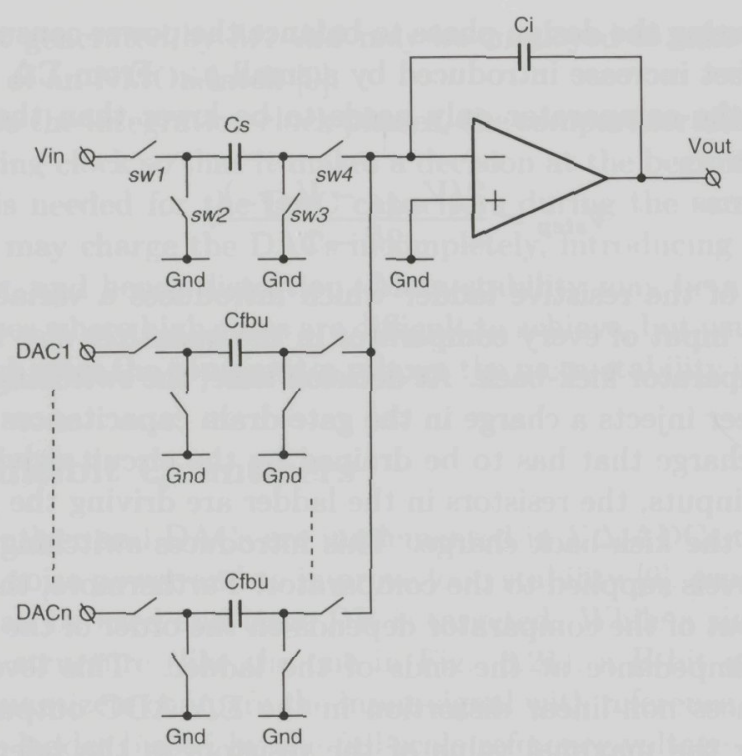

Figure 3.23: SC integrator with multibit DAC

1 for simplicity). With this structure all $C f b u$ capacitors are switched alternatively in sampling and integrating to avoid signal-dependent (or quantizer decision-dependent) switching. Since the splitting of the feedback capacitor introduces extra switching paths, the noise of the entire circuit has to be analyzed respecting the equivalence with the model of a single- $C f b$ capacitor with modulated $D A C$ voltage. Assuming that $C s$ and $C i$ remain the same, the equivalence implies that

$$
C_{f b u}=\frac{C_{f b}}{n}
$$

so, when all $n$ DACs switch in the same direction, the multibit DAC output is equal to the single-capacitor equivalent. With this reduction of capacitor size, the switching noise does not increase since the noise power of each switching $C f b u$ is referred at the input of the integrator as

$$
P_{n_{C f b u}}=\frac{4 k T}{C_{f b u}}\left(\frac{C_{f b u}}{C_{i}}\right)^{2}\left(\frac{C_{i}}{C_{s}}\right)^{2}=\frac{4 k T C_{f b u}}{C_{s}^{2}} .
$$

So the input-referred noise power is actually proportional to Cfbu, which makes the total switching noise power for all DACs to be

$$
P_{n_{n C f b u}}=\frac{4 k T n C_{f b u}}{C_{s}^{2}}=\frac{4 k T C_{f b}}{C_{s}^{2}}
$$




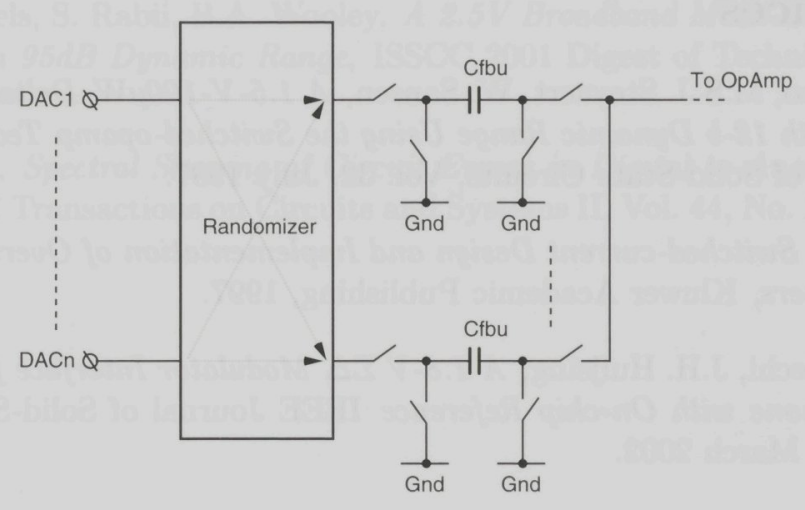

Figure 3.24: Multibit DAC with randomizing logic

showing that the noise power remains the same when the $C f b$ is split into $n$ equal $C f b u$ capacitors. Signal-dependent switching is avoided by using all the $C f b u$ capacitors during each integration phase, independent of the quantizer decision.

In practice, the unit capacitors $C f b u$ are not perfectly equal and the charge supplied by the multibit DAC on one clock becomes dependent on the quantizer output, in other words of the input signal. Because the dependency is non-linear, harmonic distortion appears at the converter's output [4]. DAC calibration is not simple in SC designs [12], so the easiest way to deal with this problem is to spread the power of the harmonic distortion in the entire sampled bandwidth (DC to $f_{s} / 2$ ) by randomizing the use of the DAC unit capacitors. A general schematic is shown in Fig. 3.24. The Randomizer block connects each of the DAC1 to DACn comparator decisions to a different capacitor $C f b u$, so two identical quantizer outputs do not generate identical DAC errors. In this way the DAC errors are modulated by the algorithm implemented by the Randomizer. If the algorithm (ideally) outputs a random sequence with infinite period the distortion introduced by DAC non-linearity is completely spread from DC to $f_{s} / 2$. In practice, using a pseudo-random algorithm usually proves to be insufficient, so data-weighted averaging (DWA) [13] is commonly used to reduce the harmonic distortion. Other randomizing methods include partitioned data averaging [14] and high-pass shaping of DAC errors [15]. 


\section{REFERENCES}

\section{References}

[1] V. Peluso, M.S.J. Steyaert, W. Sansen, A 1.5-V-100 $\mu$ W Delta-Sigma Modulator with 12-b Dynamic Range Using the Switched-opamp Technique, IEEE Journal of Solid-State Circuits, Vol. 32, July 1997.

[2] N. Tan, Switched-current Design and Implementation of Oversampling $A / D$ Converters, Kluwer Academic Publishing, 1997.

[3] O. Bajdechi, J.H. Huijsing, A 1.8-V $\Sigma \Delta$ Modulator Interface for an Electret Microphone with On-chip Reference IEEE Journal of Solid-State Circuits, Vol. 37, March 2002.

[4] S.R. Norsworthy, R. Schreier, G.C. Temes, Delta-Sigma Data Converters, IEEE Press, 1997.

[5] B.Y. Kamath, R.G. Meyer, P.R. Gray, Relationship Between Frequency Response and Settling Time of Operational Amplifiers, IEEE Journal of Solid-State Circuits, Vol. SC-9, Dec. 1974.

[6] A.L. Coban, A Low-Voltage High-Resolution Delta-Sigma Modulator, PhD Thesis, Georgia Institute of Technology, May 1998.

[7] A. Bakker, High-Accuracy CMOS Smart Temperature Sensors, PhD Thesis, Delft University of Technology, April 2000.

[8] S. Rabii, B.A. Wooley, The Design of Low-Voltage, Low-Power Sigma-Delta Modulators, Kluwer Academic Publishers, Boston, 1999.

[9] A. Marques, High Speed CMOS Data Converters, PhD Dissertation, Katholieke Universiteit Leuven, Belgium, 1999.

[10] G.C. Temes, Simple Formula for Estimation of Minimum Clock Feedthrough Error Voltage, IEE El. Letters, Vol. 22, September 1985.

[11] F. Medeiro, B.P. Verdu, A.R. Vasquez, Top-Down Design of HighPerformance Sigma-Delta Modulators, Kluwer Academic Publishers, 1999.

[12] Un-Ku Moon, J. Silva, J. Steensgaard, G.C. Temes, A Switched-Capacitor $D A C$ with Analog Mismatch Correction, IEEE International Symposium on Circuits and Systems, ISCAS 2000.

[13] R.T. Baird, T.S. Fiez, Linearity Enhancement of Multibit $\Sigma \Delta A / D$ and $D / A$ Converters using Data Weighted Averaging, IEEE Transactions on Circuits and Systems II, Vol. 42, No. 12, December 1995.

76 Systematic Design of $\Sigma \Delta$ ADCs 
[14] K. Vleugels, S. Rabii, B.A. Wooley, A 2.5V Broadband Multi-Bit $\Sigma \Delta$ Modulator with 95dB Dynamic Range, ISSCC 2001 Digest of Technical Papers, Feb. 2001.

[15] I. Galton, Spectral Shaping of Circuit Errors in Digital-to-Analog Converters, IEEE Transactions on Circuits and Systems II, Vol. 44, No. 10, October 1997. 
REFERENCES

78 Systematic Design of $\Sigma \Delta$ ADCs 


\section{4 \\ Continuous-Time Circuit Design}

Even if they do not directly map on the concept of "sampled system" normally used to describe a $\Sigma \Delta \mathrm{ADC}$, continuous-time (CT) circuits are often employed to implement the loop filters of such ADCs. This is done by taking advantage of the periodic operation of the quantizer, which makes possible the mapping of discrete-time loop filters into CT equivalents [1]. The main returns of this mapping effort are a lower power consumption of CT circuits [2] (especially at high sampling frequencies) and intrinsic anti-alias filtering [3].

Similarly with the discrete-time (DT) implementations, the main building block is the ideal low-pass filter (or integrator) which implements a transfer function with its only pole at DC:

$$
H(s)=\frac{\alpha}{s}
$$

$\alpha$ being the gain-bandwidth product (GBW) of the integrator. In CT $\Sigma \Delta$ ADC design, $\alpha$ is calculated from the filter-level NTF coefficients to map the designed NTF on the target loop topology.

The circuit techniques to build CT integrators rely on integrating a current proportional with the input signal on a capacitor and read the output voltage. A simple, passive RC filter cannot be used to implement the desired transfer function since its pole is not at DC. With passive filters, a design method which takes into account this pole shift has to be employed to map the desired NTF to the target loop topology.

The first method (Fig. 4.1, left) directly integrates the input current $I i$ on the integration capacitor $\mathrm{C} i$. This method can be applied when the input signal is a current, as is the case with certain sensor read-out circuits. The input signal source has to be able to generate the input current, a drawback which can be 

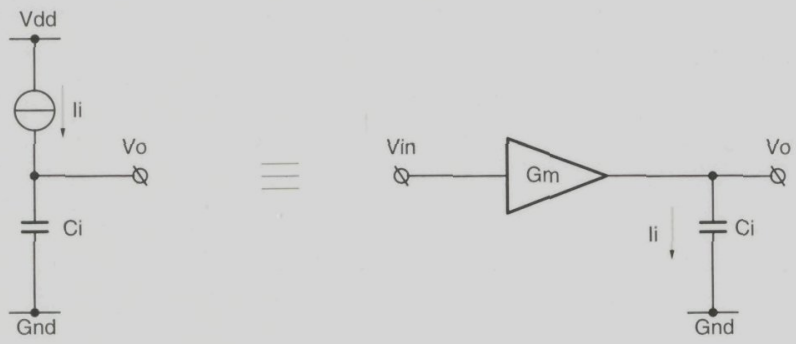

Figure 4.1: Gm-C integrator

overcome by switching to a voltage input, as in (Fig. 4.1, right). The two methods are equivalent from a functional point of view, but in the latter case the $G m$ stage can be designed to offer high input impedance. In both circuits, it is important that the next stage, connected at Vo, has a large input impedance so all the integration current $\mathrm{Ii}$ flows into $\mathrm{Ci}$. Both circuits do not contain local feedback loops so they need the lowest power for a given $\Sigma \Delta$ sampling frequency. One major drawback is the direct connection of the input current source or of the $\mathrm{Gm}$ stage to the voltage output. Variations of the integrator output are large in $\Sigma \Delta$ ADCs, and this can cause nonlinear variations of the integrated current due to finite, output-voltage dependent impedance of any CMOS current source.

The method to design high-linearity CT integrators keeps one end of the voltage-to-current converter at a fixed voltage with an active circuit, as is the case with $R i$ in Fig. 4.2. With an ideal amplifier, the transfer function of this integrator is

$$
H(s)=\frac{1}{s R_{i} C_{i}}
$$

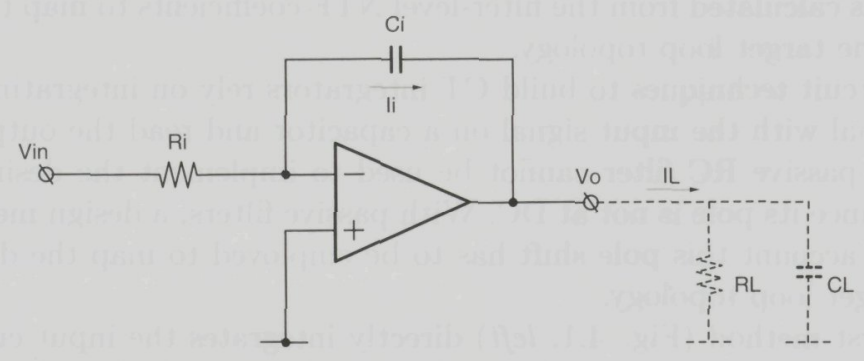

Figure 4.2: Active RC integrator 
showing that the integrator bandwidth is given only by the passive components. The operational amplifier ideally holds its negative input to ground, hence the integrated current is only dependent on the input voltage Vin and the conversion resistor Ri. Also, for a CMOS opamp, the input bias current is null, hence all the input current is integrated on $\mathrm{Ci}$. Two main advantages make this circuit the choice of most CT $\Sigma \Delta$ ADC designers: linearity is only limited by the opamp's performance [4] and not by second-order effects like in the circuits presented above, and by its low output impedance it is capable to supply the load current $I L$ generally required by the next stage. The disadvantage of increased supply current required to keep the amplifier stable is usually not a major drawback in applications targeting high speed, resolution and linearity.

The analyses presented in this chapter are focused on differential implementations of the active RC integrator in Fig. 4.2. Current-input implementations of this integrator are possible but since they are not used in mainstream designs (because of a lack of highly-linear current-mode signal sources) they will not be analyzed here. The voltage-input, active-RC integrator is analyzed in detail in Section 4.1. An example of building a quantizer suited for use in CT $\Sigma \Delta$ ADCs is given in Section 4.2. In Section 4.3 different DAC topologies are discussed.

\subsection{Active RC Integrator}

\subsubsection{Topology}

A fully-differential active-RC integrator for use in $\Sigma \Delta$ ADC design is shown in Fig. 4.3. Apart from the signal input path, Vin with $\mathrm{V} / \mathrm{I}$ conversion performed by $R i$, there is a second path which implements the $\Sigma \Delta$ DAC, in this case only one-bit, with the pair of resistors $R d a c$. The current from both paths is summed

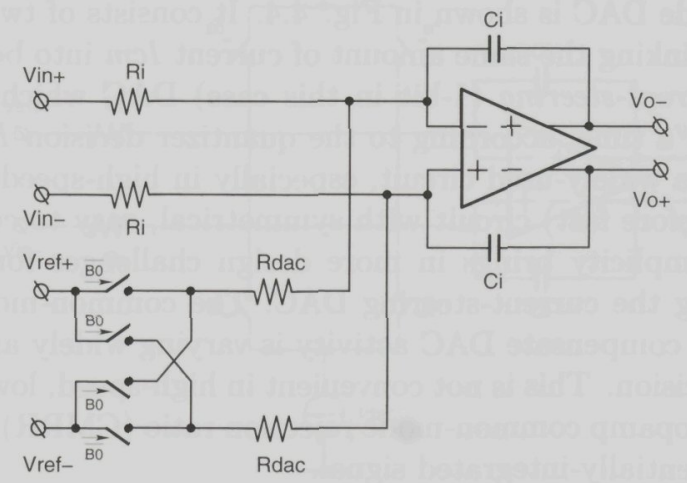

Figure 4.3: Active $R C$ integrator with $D A C$ resistors 


\section{Continuous-Time Circuit Design}

and integrated on $\mathrm{C} i$. The opamp keeps a null voltage difference between its inputs, hence the integrated current is

$$
I_{C i}=\frac{V_{i n}^{+}-V_{i n}^{-}}{2 R_{i}} \pm \frac{V_{r e f}^{+}-V_{r e f}^{-}}{2 R_{d a c}}
$$

The sign of the summation is decided based on the decision of the $\Sigma \Delta$ quantizer, represented in the schematic by the logic bit $B O$. If the decision $B O$ is logicHigh, it means the output of the integrator is too large and Vref is switched to reduce the output voltage.

The noise power at the input of the integrator is given by the input and DAC resistors and the $g_{m}$ of the opamp's first stage

$$
P_{\text {CTnoise }}=8 k T B W\left(R_{i}+\frac{R_{i}^{2}}{R_{d a c}}+\frac{\gamma}{4 g_{m}}\right)
$$

with $\gamma$ being the noise enhancement factor for short-channel transistors [5]. The noise densities of both the resistors and of the opamp are multiplied with the signal bandwidth $B W$. As opposed to SC integrators, there is no noise aliasing since no noise sampling is taking place. The noise of the DAC resistors is referenced back to the input through the squared value of the $R_{i}$ resistors. The noise in the reference voltage Vref can be added in the same manner in the final design.

The integrator using DAC resistors can be extended for multi-bit DACs by adding DAC paths, independently connected to the same Vref. Although structurally possible, there is one aspect of multi-bit DACs which cannot be handled with ease using a resistor-based DAC, namely calibration. High-precision calibration is however easy to implement on current sources, especially in MOS technologies [6]. The DAC resistors can be replaced with constant current source since they are connected to a constant voltage Vref, and one possible topology for a current-mode DAC is shown in Fig. 4.4. It consists of two common-mode current sources sinking the same amount of current $I \mathrm{~cm}$ into both opamp input nodes and a current-steering (1-bit in this case) DAC which is connected to only one node at a time, according to the quantizer decision BO. The currentsteering DAC is a widely-used circuit, especially in high-speed designs, since it is a simple (therefore fast) circuit with symmetrical, easy to control switching. However, this simplicity brings in more design challenges for the rest of the circuit containing the current-steering DAC. The common-mode current sunk by the opamp to compensate DAC activity is varying widely and dependent on the quantizer decision. This is not convenient in high-speed, low-voltage designs where a reduced opamp common-mode rejection ratio (CMRR) can significantly distort the differentially-integrated signal.

To correct this issue, a completely differential current-mode DAC would be preferred. Since it is difficult to design a floating current source which can be 


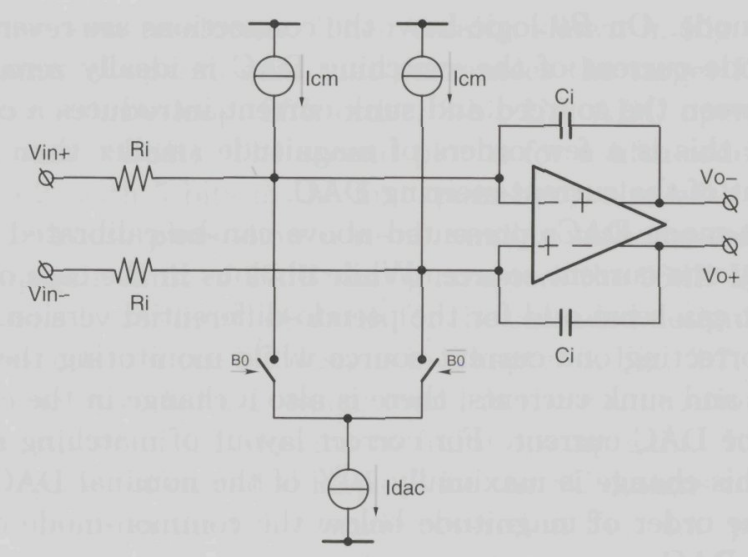

Figure 4.4: Active RC integrators with current-steering $D A C$

switched between the two inputs of the opamp, a pseudo-differential approach can be made, as shown in Fig. 4.5. In this case two current sources are used, one sourcing and one sinking. They are alternately connected to one or the other of the opamp inputs, according to quantizer decision B0. Even if no floating current source is used, the overall effect is the same. On B0 logic-High, Idact is sourced into the non-inverting opamp input node and $I d a c$-is sunk from the

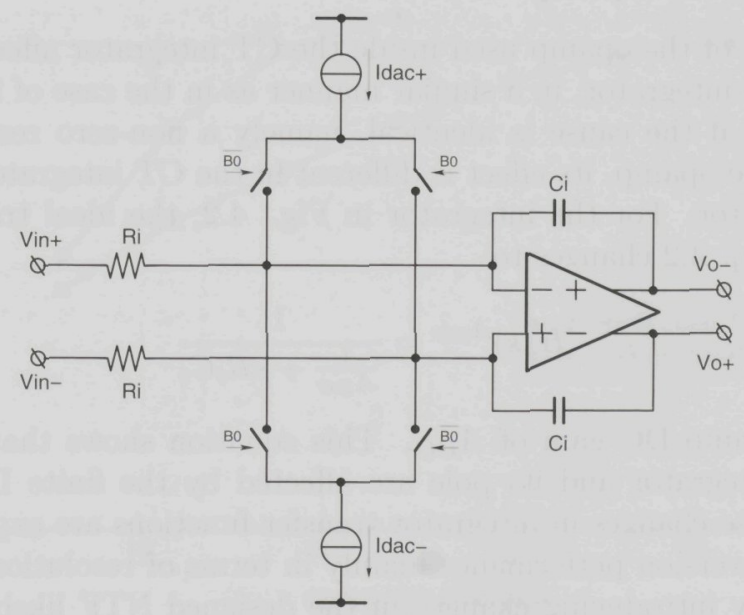

Figure 4.5: Active RC integrator with differential current-mode DAC 
inverting input node. On BO logic-Low, the connections are reversed, therefore the common-mode current of the switching DAC is ideally zero. In practice, a difference between the sourced and sunk current introduces a common-mode component, but this is a few orders of magnitude smaller than the commonmode component of the current-steering DAC.

Both current-mode DACs presented above can be calibrated by correcting the value of only one current source. While obvious in the case of the currentsteering DAC, it can seem odd for the pseudo-differential version. In the latter case, by only correcting one current source while monitoring the difference of the two sourced and sunk currents, there is also a change in the common-mode component of the DAC current. For correct layout of matching multiple DAC cells like this, this change is maximally $10 \%$ of the nominal DAC cell current, which is still one order of magnitude below the common-mode current of the current-steering DAC.

\subsubsection{Effects of Circuit Non-Idealities}

In comparison with a switched-capacitor (SC) integrator, the only active element in a CT integrator with resistive DAC is the operational amplifier. In such integrator topologies the opamp is the circuit element defining integrator's performance, assuming highly linear on-chip passive components are available, i.e. polysilicon resistors and metal/metal or poly/poly capacitors. But, similarly with the SC integrators, the effects of these circuit non-idealities can be analysed and limits for them can be derived that guarantee a target performance.

\section{Finite, Nonlinear Opamp Gain}

The finite gain of the opamp used inside the CT integrator affects the transfer function of the integrator, in a similar manner as in the case of SC integrators. However, even if the cause is identical, namely a non-zero residual signal at the input of the opamp, its effect is different in the CT integrator compared to the SC integrator. For the integrator in Fig. 4.2, the ideal transfer function described in Eq. 4.2 changes to

$$
H(s)^{A_{D C}}=\frac{1}{\frac{1}{A_{D C}}+s R_{i} C_{i}}
$$

for a finite opamp DC gain of $A_{D C}$. This equation shows that both the DC gain of the integrator and its pole are affected by the finite DC gain of the amplifier. These changes in integrator transfer functions are expected to cause loss of $\Sigma \Delta$ conversion performance mainly in terms of resolution, i.e. dynamic range (DR), by introducing changes in the designed NTF likely to produce a leakage of quantization noise inside the signal bandwidth. The performance of two designs has been simulated to analyse the effect of finite opamp gain

\section{Systematic Design of $\Sigma \Delta$ ADCs}




\subsection{Active RC Integrator}

on overall conversion resolution. Their results, shown in Fig. 4.6, are used to extract the minimal opamp gain that guarantees the target $\mathrm{DR}$ of $96 \mathrm{~dB}$. The first plot is for a single-loop, fifth order, 3-bit $\Sigma \Delta$ ADC operated at 32 times oversampling ratio (OSR). The second plot is for a fifth-order cascade of two loops(orders 3-2, with 5 bits in the first quantizer), also with three bits in the last quantizer. These plots show a result similar with the case of SC designs, with an opamp gain limit of $20 \mathrm{~dB}$ that guarantees the $96 \mathrm{~dB}$ DR for the singleloop design and a larger limit, of $40 \mathrm{~dB}$, for the cascaded design. Compared with the results presented for the SC designs in Chapter 3, Fig. 3.7, the lower opamp gain limit for the cascaded CT design found in these simulations does not show a reduced sensitivity of $\mathrm{CT}$ designs to finite opamp gain. It rather confirms that a larger number of cascaded loops ( 3 for the $\mathrm{SC}$ designs covered in Fig. 3.7 compared to 2 loops in the CT cascade covered in Fig. 4.6) produces a design more sensitive to finite opamp gain. An overall comparison of the results in Fig. 3.7 and Fig. 4.6 shows that both $\mathrm{SC}$ and active RC $\Sigma \Delta$ ADCs are sensitive in the same measure to finite opamp gain and in both cases the cascaded designs are more sensitive than single-loop designs. The results may vary with the aggressiveness of the NTF used in different designs but it is really difficult (if not impossible) to find a cascaded design less sensitive to finite opamp gain than a single-loop design.

Continuous-time integrators are also expected to be sensitive to opamp gain

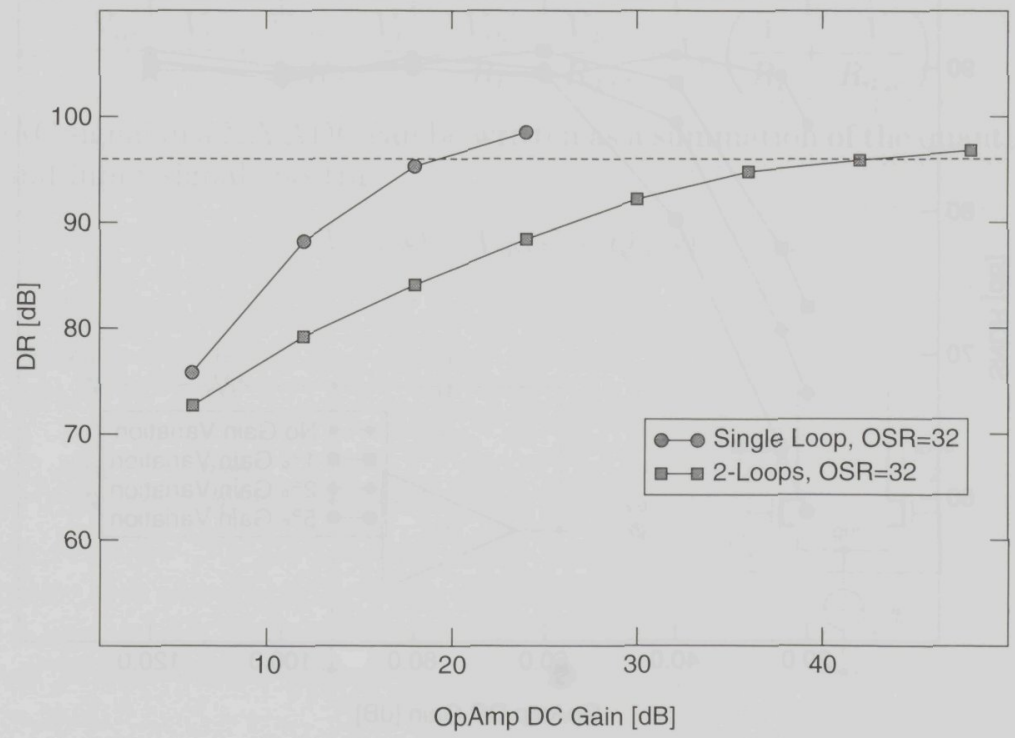

Figure 4.6: Effect of finite opamp gain over dynamic range in CT $\Sigma \Delta$ ADCs 
variation caused by the dependence of output impedance on the output voltage. This variation, connected with a finite nominal gain, maintains a signaldependent residual voltage at the opamp's input. The residual voltage is subtracted from the input signal, and only this difference is converted to a current on the input resistor Ri (see Fig. 4.2). As it has been explained in Chapter 3, the opamp's voltage gain variation can be modeled as a piecewise-linear function of the output voltage $V_{\text {out }}$

$$
A_{D C}\left(V_{\text {out }}\right)=A_{D C}(0)\left(1-\frac{\left|V_{\text {out }}\right|}{\max \left(\left|V_{\text {out }}\right|\right)} \Delta A\right)
$$

Its piecewise-linear behavior coupled with the dependence of the output voltage on the input signal introduces non-linearity in the overall $\Sigma \Delta$ conversion which reduces the peak signal-to-noise+distortion (SNDR) value. This reduction depends on both peak gain variation $\Delta A$ and nominal voltage gain (at zero output) $A_{D C}(0)$. A graph showing the effect of these complex dependencies for the first integrator in a single-loop, fifth-order, 3-bit design has been extracted from simulations and is given in Fig. 4.7 as four plots showing the peak SNDR at different $A_{D C}(0)$ values for gain variation $\Delta A$ ranging from $0 \%$ to $5 \%$. The control curve, marked No Gain Variation, shows that for an opamp gain of $24 \mathrm{~dB}$ with $0 \%$ gain variation the peak SNDR of $90 \mathrm{~dB}$ is reached. A gain of $40 \mathrm{~dB}$ is needed if the gain variation is $1 \%$, and $60 \mathrm{~dB}$ if the variation is larger.

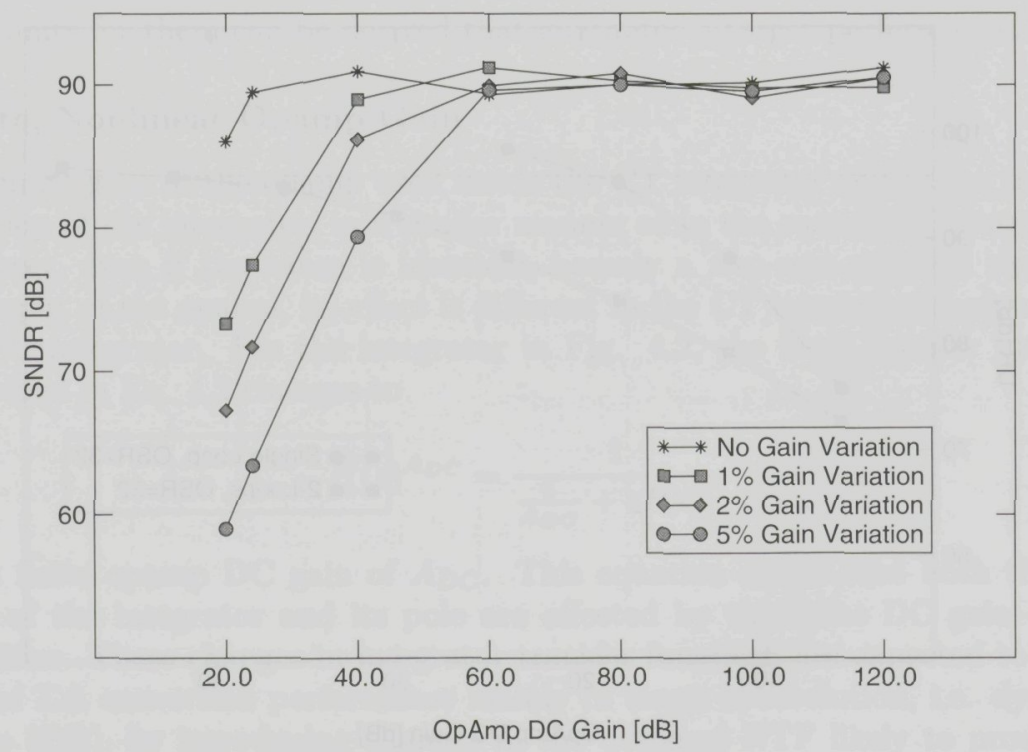

Figure 4.7: Peak SNDR decrease through signal-dependent opamp gain 
Compared with the $\mathrm{SC}$ designs covered in Chapter 3, Fig. 3.8, these results show an increased sensitivity of CT designs to non-linear variation of integrator opamp gain. Approximately 93dB distortion-limited peak SNDR is reached for a SC design with 40dB nominal opamp gain and $5 \%$ gain variation, while only $80 \mathrm{~dB}$ peak SNDR is reached for a CT design under the same conditions.

\section{Finite, Nonlinear OpAmp Transconductance}

Although used as a voltage-input, voltage-output circuit, the active-RC CT integrator actually integrates a current, $I_{i}$ in Fig. 4.8. The previous sub-Section covered the non-linearity of this current introduced by the finite, nonlinear output impedance of a CMOS amplifier. The other major non-linearity of the CMOS amplifier, introduced by its finite and strongly nonlinear transconductance $g_{m}$, is analyzed here. Since the integrator functions with current signals, the effect of the transconductance can be de-coupled from the effect of the output impedance. Furthermore, a simplified model of a single-stage amplifier can be used for this analysis, yielding results that can also be applied to more complex opamp designs by replacing the $g_{m}$ in this model with the equivalent $g_{m}$ of a multi-stage, correctly-compensated (approximately single-pole behavior) amplifier.

For the integrator in Fig. 4.8 with a finite opamp transconductance, the integrated current depends on both the input voltage $V_{i n}$, the DAC voltage $V_{d a c}$ and the non-zero residual voltage $V_{r}$

$$
I_{i}=\frac{V_{i n}-V_{r}}{R_{i}}+\frac{V_{d a c}-V_{r}}{R_{d a c}}=\frac{V_{i n}}{R_{i}}+\frac{V_{d a c}}{R_{d a c}}-V_{r}\left(\frac{1}{R_{i}}+\frac{1}{R_{d a c}}\right)
$$

The DAC signal in a $\Sigma \Delta \mathrm{ADC}$ can be written as a summation of the quantization noise and input signal spectra

$$
V_{d a c}(s)=V_{i n}(s)+Q_{n}(s)
$$
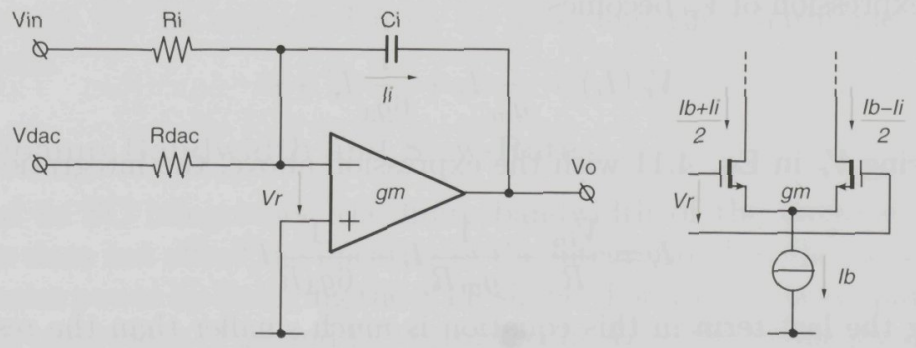

Figure 4.8: Active RC integrator with resistive feedback path and finite opamp transconductance 


\section{Continuous-Time Circuit Design}

Because inside the conversion bandwidth the power of the shaped quantization noise is much smaller than the signal power, the DAC signal can be approximated by only the input signal at the frequencies of interest.

$$
V_{d a c} \approx V_{i n}
$$

With this simplified feedback signal, the equivalent $\mathrm{V} / \mathrm{I}$ conversion resistor can be defined as

$$
\frac{1}{R}=\frac{1}{R_{i}}+\frac{1}{R_{d a c}}
$$

and Eq. 4.7 becomes

$$
I_{i}=\frac{V_{\text {in }}}{R}-\frac{V_{r}}{R}
$$

The source of non-linearity is the residual voltage $V_{r}$ produced by the finite, non-linear $g_{m}$ integrating the current $I_{i} . V_{r}$ can be written as function of (only) $I_{i}$ in a truncated Taylor series around $V_{r}=0$

$$
V_{r}\left(I_{i}\right)=V_{r}(0)+\frac{\partial V_{r}}{\partial I_{i}} I_{i}+\frac{1}{2} \frac{\partial^{2} V_{r}}{\partial I_{i}^{2}} I_{i}^{2}+\frac{1}{6} \frac{\partial^{3} V_{r}}{\partial I_{i}^{3}} I_{i}^{3}+\cdots
$$

The first term is zero if the opamp is offset-free, $V_{r}(0)=0$. For an input stage shown in Fig. 4.8 (right) functioning balanced and isolated from ground by the tail current source $I b$, the second derivative always results null because of the differential signal processing and identical input transistors. The first derivative is the inverse of the nominal $g_{m}$, while the third partial derivative is the inverse of a transconductance-like parameter (although without physical significance)

$$
\begin{aligned}
& \frac{\partial V_{r}}{\partial I_{i}}=\frac{1}{g_{m}} \\
& \frac{\partial^{2} V_{r}}{\partial I_{2}^{2}}=0 \\
& \frac{\partial^{3} V_{r}}{\partial I_{2}^{3}}=\frac{1}{g_{3}}
\end{aligned}
$$

and the expression of $V_{r}$ becomes

$$
V_{r}\left(I_{i}\right)=\frac{1}{g_{m}} I_{i}+\frac{1}{6 g_{3}} I_{i}^{3}+\cdots
$$

By replacing $V_{r}$ in Eq. 4.11 with the expression above, the integration current becomes

$$
I_{i}=\frac{V_{i n}}{R}-\frac{1}{g_{m} R} I_{i}-\frac{1}{6 g_{3} R} I_{i}^{3}
$$

Assuming the last term in this equation is much smaller than the rest, an expression of the linear $I_{i}$ as a function of $V_{i n}$ results

$$
I_{i}^{1 s t}=\frac{V_{i n}}{R} \frac{g_{m} R}{1+g_{m} R}=\frac{V_{i n} g_{m}}{1+g_{m} R}
$$


which in turn is used to calculate the cubed component of $I_{i}$ as

$$
I_{i}^{3 r d}=\frac{\left(I_{i}^{1 s t}\right)^{3}}{6 g_{3} R}=\frac{V_{i n}^{3} g_{m}^{3}}{6 g_{3} R\left(1+g_{m} R\right)^{3}}
$$

If a single-tone input signal is applied,

$$
V_{\text {in }}=V_{A} \cos (\omega t)
$$

by replacing the expression above in Eq. 4.17 and considering the trigonometric property of the cosine

$$
\cos ^{3}(x)=\frac{3}{4} \cos (x)+\frac{1}{4} \cos (3 x)
$$

the third and main spectral components of the integrated current become

$$
\begin{aligned}
& H_{1}=\frac{V_{A} g_{m}}{1+g_{m} R}+\frac{3}{4} H_{3} \\
& H_{3}=\frac{1}{4} \frac{V_{A}^{3} g_{m}^{3}}{6 g_{3} R\left(1+g_{m} R\right)^{3}}
\end{aligned}
$$

The second term in $H_{1}$ is much smaller than the first one, so it can be ignored in the summation. If the third harmonic is the dominant distortion component (as assumed in this demonstration by ignoring the higher-order terms in the Taylor series in Eq. 4.12), total harmonic distortion can be approximated by the third harmonic distortion

$$
H D_{3}=\frac{H_{3}}{H_{1}}=\frac{V_{A}^{2} g_{m}^{2}}{24 g_{3} R\left(1+g_{m} R\right)^{2}} \approx \frac{V_{A}^{2}}{24 g_{3} R^{3}}
$$

For CMOS opamps, expressions for both $g_{m}$ and $g_{3}$ can be extracted based on the sizing and operating point of the input devices. Since this distortion source is lower-limiting the opamp transconductance for a target harmonic distortion (which in turn would decrease the peak SNDR) the expressions of $g_{m}$ and $g_{3}$ will be calculated in a following sub-Section where the power consumption of the integrator will be estimated from its noise and third harmonic distortion performance.

\section{Finite Opamp Bandwidth and Slew-Rate}

Compared to SC integrators, the finite bandwidth of the amplifier in a CT integrator does not affect the linearity of the integration but the in-band quantization noise power by changing the NTF shape. For a single-pole opamp with a unity-gain bandwidth of $\omega_{0}$ and DC voltage gain $A_{D C}$, the frequency-dependent voltage gain

$$
A(s)=\frac{A_{D C}}{1+\frac{s A_{D C}}{\omega_{0}}}
$$


can be used in Eq. 4.5 to find the transfer function of a CT integrator containing the bandwidth-limited opamp

$$
H(s)^{G B W}=\frac{1}{\frac{1}{A(s)}+s R_{i} C_{i}}=\frac{1}{\frac{1}{A_{D C}}+s\left(\frac{1}{\omega_{0}}+R_{i} C_{i}\right)}
$$

The equation above shows that, in order to have a good approximation of the ideal integrator function,

$$
H(s)^{i \text { deal }}=\frac{1}{s R_{i} C_{i}}
$$

not only the DC gain $A_{D C}$ should be much larger than $1 /\left|s R_{i} C i\right|$, but also the unity-gain bandwidth of the opamp should be much higher than the unity-gain bandwidth of the integrator

$$
\omega_{0} \gg \frac{1}{R_{i} C_{i}}
$$

To compare the result in the equation above with the sampling frequency $f_{C L K}$ of the $\Sigma \Delta$ ADC, the value of the loop coefficient associated with the integrator

$$
a_{i}=\frac{1}{R_{i} C_{i} f_{C L K}}
$$

(assuming non-return-to-zero design) should be used. For typical $a_{i}$ values of 0.1 to 0.3 , results that

$$
\omega_{0} \geq f_{C L K} \cdots 3 f_{C L K}
$$

The exact value also depends on the aggressiveness of the NTF (which decreases tolerance to pole errors, see Chapter 2), the margin between the in-band physical noise and quantization noise power (which can be increased to cover the in-band quantization noise leakage) and also of the fill factor for a return-to-zero (RTZ) DAC shape (which changes the integrator bandwidth on the feedback path, $1 / R_{d a c} C_{i}$ becoming $\delta / R_{d a c} C_{i}$, with $\delta$ the RTZ fill factor).

The second issue associated with a limited opamp bias current is the slewing of the amplifier's output stage. Since the signal processed is the integrated current (supplied by the output stage of the amplifier), a limitation of this signal would introduce distortion in the overall A/D conversion. Therefore, a hard limit can be deduced for the biasing current of the output stage of the opamp as the peak of the integrated current during nominal operation of the $\Sigma \Delta$ ADC. This is usually not a very restrictive limit. As an example, for a fifth-order, one-bit, fully-differential design biased at $1.8 \mathrm{~V}$ and attaining $96 \mathrm{~dB}$ DR in a signal bandwidth of $100 \mathrm{kHz}$, the integration and DAC resistors of the first integrator result from the noise budget at $24 k \Omega$. With the input signal at full swing of $1.8 \mathrm{~V}$ and added to the DAC signal, the peak signal current results $75 \mu \mathrm{A}$, a value not difficult to supply to a design with the target performance as high as listed above. Hence, not reaching slewing conditions is not difficult in 
an active-RC integrator. Even in a design with current-mode feedback (where slewing inherently occurs for very short times during DAC switching) the effects of the slewing are negligible for distortion levels as low as $-100 \mathrm{dBc}$.

\subsubsection{Operational Amplifier Topologies}

Given the tight requirements on opamp gain linearity in CT integrators observed in one of the previous sub-Sections of this chapter, the choice of the opamp inside a CT integrator is dictated mainly by its DC gain and its (sufficiently linear) output voltage range. Bandwidth comes in second since the fast switching is by far not as important as in SC designs. The ratio of bandwidth versus power should also be optimized but not on the expense of the first requirement.

One topology which offers a large DC gain with as little power consumption as possible is the single-stage opamp in Fig. 4.9. Both its input pair M1-M2 and its active loads $M 7-M 8$ are cascoded to increase the output impedance. Hence, compared to a non-cascoded version (like the one in Chapter 3, Fig. $3.15)$ the DC gain is boosted by the voltage gain of the cascode transistors, $\mu_{M 3}=g_{m_{M 3}} / g_{d s_{M 3}}$ and $\mu_{M 5}=g_{m_{M 5}} / g_{d s_{M 5}}$, to

$$
A_{D C}=\frac{g_{m_{M 1}}}{\frac{g_{d s_{M 1}}}{\mu_{M 3}}+\frac{g_{d s_{M 3}}}{\mu_{M 5}}}
$$

The amplifier bandwidth is given by the transconductance of the input pair $g_{m_{M 1}}$ and its open-loop load capacitor $C_{o l}$

$$
U G B=\frac{g_{m_{M 1}}}{2 \pi C_{o l}}
$$

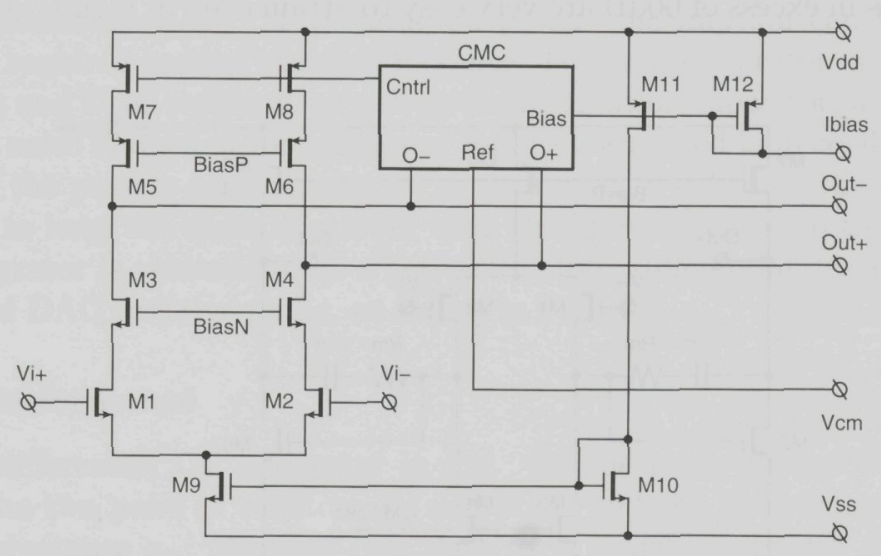

Figure 4.9: Single-stage opamp with high DC gain 
The non-zero transit time through the cascode transistors introduces a second pole which sets an upper limit for the bandwidth of a stable amplifier.

The main disadvantage of this amplifier lies in its output range. The loss of two additional saturation voltages on top of the ones for $M 1, M 7$ and especially M9 (with large saturation voltage for good common-mode rejection) can be a costly price for the low power consumption, especially in high-speed CMOS processes where the $V d d-V s s$ can be as low as $1.8 \mathrm{~V}$ or even $1.0 \mathrm{~V}$. At such signal range, the loss of two saturation voltages (which, as opposed to the threshold voltages, do not scale with the gate oxide thickness in CMOS processes) means a reduction of the output range by as much as $20 \%$. In a $\Sigma \Delta$ ADC, this means more than $20 \%$ larger integration capacitors to reduce the output swing accordingly.

The common-mode is set by a common-mode control block $(C M C)$ which, in case linearity is critical, should be a CT design (like the one shown in Chapter 3, Fig. 3.16). The $\mathrm{SC}$ version of the $\mathrm{CMC}$ can introduce un-wanted effects in the conversion linearity by sourcing high peak currents from the opamp, pushing it into slewing for significant periods of time.

In the case when output range should be maximized, a two-stage design with a non-cascoded, class-A output amplifier should be used. The simplest topology of this amplifier is given in Fig. 4.10. The first stage is built with the input pair M1-M2 and their active loads M3-M4. It drives the second stage built with the gain transistors $M 5-M 6$ and the active loads $M 7-M 8$. A topology with NMOS input pair can also be designed, but the one in Fig. 4.10 has the advantages of a better common-mode rejection due to increased isolation of the PMOS input pair, NMOS gain devices in the output stage where a large transconductance is required, and better-matching PMOS active loads in the output stage.

DC gains in excess of $60 \mathrm{~dB}$ are very easy to attain even in high-speed CMOS

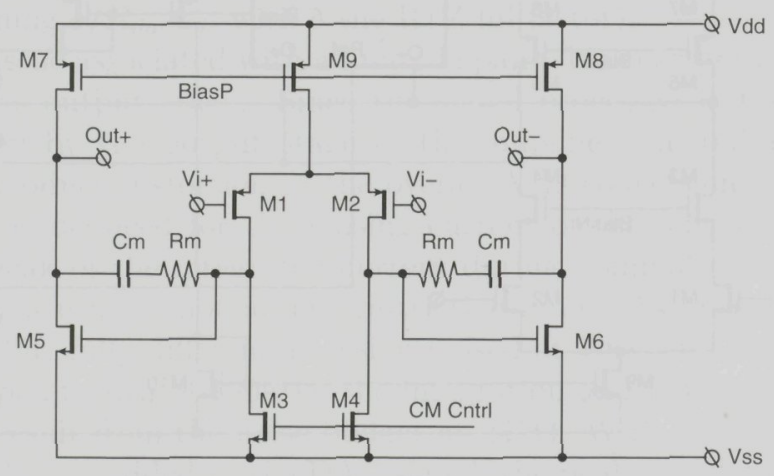

Figure 4.10: Class-A, Miller-compensated, two-stage opamp 
due to the two-stage design

$$
A_{D C}=\frac{g_{m_{M 1}}}{g_{d s_{M 1}}+g_{d s_{M 3}}} \frac{g_{m_{M 6}}}{g_{d s_{M 6}}+g_{d s_{M 8}}}
$$

For a well-designed opamp with 60-degree phase margin under a given capacitive load, the bandwidth of the amplifier is given by the input transconductance and the Miller capacitor $\mathrm{Cm}[7]$

$$
U G B=\frac{g_{m_{M 1}}}{2 \pi C_{m}}
$$

A well-designed amplifier is placing the non-dominant pole introduced at the output by the load capacitor $C_{o l}$

$$
p_{2}=\frac{g_{m_{M 6}}}{2 \pi C_{o l}}
$$

at a frequency at least two times larger than the target UGB. This constraint usually means large power consumption in the output stage, the main disadvantage of this opamp topology.

The common-mode control can be implemented as explained with the singlestage opamp topology presented before. The best point to close the commonmode feedback loop is the common gate of $M 3-M 4$ since they are probably the least-constrained devices in this design.

The resistors $R m$ in series with the Miller capacitors compensate for the positive zero produced by the Miller compensation of a low-gain stage, M6.

\subsubsection{Power Consumption Analysis}

A good estimate of the CT integrator's power consumption can be obtained from the required opamp transconductance. For each integrator in a $\Sigma \Delta$ ADC, the input and DAC resistors, as well as the integration capacitor, are calculated from the noise budged of the integrator and its associated loop coefficients. The values of the passive components are in turn used to calculate the opamp's $g_{m}$ required to keep the distortion lower than the limit allocated to the integrator. The integrator is assumed to be a active-RC, fully differential design with both input and DAC resistive paths, as shown in Fig. 4.3.

\section{Noise Performance}

For the differential CT integrator in Fig. 4.3 the noise budget is distributed among the two pairs of resistors $R_{i}$ and $R_{d a c}$, and the operational amplifier's transconductance $g_{m}$, resulting (Eq. 4.4), for a signal bandwidth $B W$,

$$
P_{\text {CTnoise }}=8 k T B W\left(R_{i}+\frac{R_{i}^{2}}{R_{d a c}}+\frac{\gamma}{4 g_{m}}\right)
$$


The equation above can be written in a more compact form if the expressions connecting the $\Sigma \Delta$ loop coefficients with the passive circuit components are used (see Chapter 2, Fig. 2.9)

$$
\begin{aligned}
& a=\frac{1}{R_{i} C_{i} f_{C K}} \\
& f=\frac{R_{i}}{R_{d a c}}
\end{aligned}
$$

to write $P_{C T n o i s e}$ only as function of $C_{i}$ and $R_{i}$

$$
P_{C T \text { noise }}=\frac{8 k T B W}{a C_{i} f_{C K}}\left(1+f+\frac{\gamma}{4 g_{m} R_{i}}\right)
$$

To quickly estimate $C_{i}$, the last term of the equation above can be ignored or be allocated a (small) fraction of the noise budget. Knowing $C_{i}$, the resistors can be calculated and used to calculate $g_{m}$ from the distortion specification. Alternatively, an optimized set of circuit parameters can be calculated if first the distortion specification is used to extract an expression for the $g_{m} R_{i}$ product, free from other circuit parameters.

\section{Distortion Performance}

Starting from the result of distortion analysis given in Eq. 4.21 and assuming the opamp has a large $g_{m}\left(g_{m} R \gg 1\right)[4]$

$$
H D_{3}=\frac{V_{A}^{2}}{24 g_{3} R^{3}}
$$

the distortion results to only depend on the non-linear component of the opamp's transconductance, $g_{3}$. The expression of $g_{3}$ is calculated as the third derivative of the residual input voltage which, for an input pair as shown in Fig. 4.8 with transistors sized $W / L$ in strong inversion, is

$$
V_{r}^{S I}=\sqrt{\frac{I_{b}}{2 K_{P} W / L}}\left(\sqrt{1+\frac{I_{i}}{I_{b}}}-\sqrt{1-\frac{I_{i}}{I_{b}}}\right)
$$

while, for weak inversion transistors, it is

$$
V_{r}^{W I}=n V_{T} \ln \left(\frac{I_{b}+I_{i}}{I_{b}-I_{i}}\right)
$$

Derivatives of these expressions yield the transconductance components $g_{m}$ and $g_{3}$. For strong inversion they are

$$
\begin{aligned}
& g_{m}^{S I}=\left(\frac{\partial V_{r}}{\partial I_{i}}\right)^{-1}=\sqrt{\frac{1}{2 K_{P}(W / L) I_{b}}}=\frac{I_{b}}{V_{g t}} \\
& g_{3}^{S I}=\left(\frac{\partial^{3} V_{r}}{\partial I_{i}^{3}}\right)^{-1}=\frac{3}{4 I_{b}^{3}} \sqrt{\frac{I_{b}}{2 K_{P}(W / L)}}=\frac{3}{4} \frac{1}{g_{m}^{S I} I_{b}^{2}}
\end{aligned}
$$

94 Systematic Design of $\Sigma \Delta$ ADCs 
with $V_{g t}=V_{g s}-V_{T}$. For weak inversion the expressions are

$$
\begin{gathered}
g_{m}^{W I}=\frac{I_{b}}{2 n V_{T}} \\
g_{3}^{W I}=\frac{I_{b}^{3}}{4 n V_{T}}
\end{gathered}
$$

With the derivatives calculated above and considering the expression of $R$ (Eq. 4.10), the third harmonic distortion becomes, for strong inversion transistors,

$$
H D_{3}^{S I}=\frac{V_{A}^{2} V_{g t}}{32 R_{i}^{3} I_{b}^{3}}\left(1+\frac{R_{i}}{R_{d a c}}\right)
$$

and, respectively, for weak inversion,

$$
H D_{3}^{W I}=\frac{V_{A}^{2} n V_{T}}{6 R_{i}^{3} I_{b}^{3}}\left(1+\frac{R_{i}}{R_{d a c}}\right)
$$

\section{Required OpAmp Transconductance}

From the equations above and using Eqs. 4.34, 4.39 and 4.40, a compact expression of the required $g_{m}$ for transistors in strong inversion

$$
g_{m}^{S I}=\frac{1}{R_{i}} \sqrt[3]{\frac{V_{A}^{2}(1+f)}{32 V_{g t}^{2} H D_{3}}}
$$

and, respectively, for weak inversion

$$
g_{m}^{W I}=\frac{1}{R_{i}} \sqrt[3]{\frac{V_{A}^{2}(1+f)}{48 n^{2} V_{T}^{2} H D_{3}}}
$$

Both expressions yield an expression for the product $g_{m} R_{I}$ which is free of other design parameters

$$
g_{m}=\frac{K_{g m}}{R_{i}}
$$

The factor $K_{g m}$ can be calculated before the optimization of each integrator takes place, from $\Sigma \Delta$ loop coefficients, performance requirements, MOS transistor parameters, and a design decision for $V_{g t}$ when strong inversion is used.

With $K_{g m}$, the integration capacitor $C_{i}$ can be calculated from the noise budget

$$
C_{i}=\frac{8 k T B W}{a P_{C T \text { noise }} f_{C K}}\left(1+f+\frac{\gamma}{4 K_{g m}}\right)
$$

and, using Eq. 4.34, the resistors $R_{i}$ and $R_{d a c}$ are calculated. With $R_{i}, g_{m}$ is calculated from Eq. 4.45 or directly from Eqs. 4.43 and 4.44 . 


\section{Continuous-Time Circuit Design}

\subsection{Quantizer}

The design of the quantizer in a CT $\Sigma \Delta$ ADC is mainly affected by the decision on using a RTZ DAC pulse or a NRZ. The difference lies in the quantizer timing since a RTZ DAC pulse is similar with a SC DAC while a NRZ DAC pulse requires the quantizer decision to be available as close as possible to the beginning of a sampling clock period to reduce excess loop delay [8].

If a RTZ DAC pulse is used, the full RTZ period (usually half a clock cycle) is available for the quantizer to make a decision and supply it to the DAC. Because of this, the quantizer in RTZ designs can have the same topology as a quantizer designed for a SC $\Sigma \Delta$ ADC. The comparators building these quantizers are specified in terms of hysteresis and meta-stability. Both these parameters, if larger than certain limits, can decrease the DR by modulating the high-frequency shaped quantization noise back at low frequencies. In multi-bit quantizers, the offset of each constituent comparator should be smaller than the reference voltage step to guarantee a correct NTF implementation.

If a NRZ DAC is designed, the DACs are used during the entire clock cycle and no spare time is available. In this case, the quantizer has to switch as fast as possible and supply its decision in a short and (ideally) signal-independent time. A non-zero, constant excess loop delay changes the DAC pulse waveform and therefore it affects the NTF shape, producing in-band quantization noise leakage. A signal-dependent excess loop delay has an effect similar with the intersymbol interference (see Chapter 2), increasing low-frequency noise power by leakage and distorting the output signal by making the DAC pulse waveform signal-dependent.

A fast comparator can be built with a PMOS input stage and an NMOS latch, as shown in Fig. 4.11. The voltage-input comparator stage is built

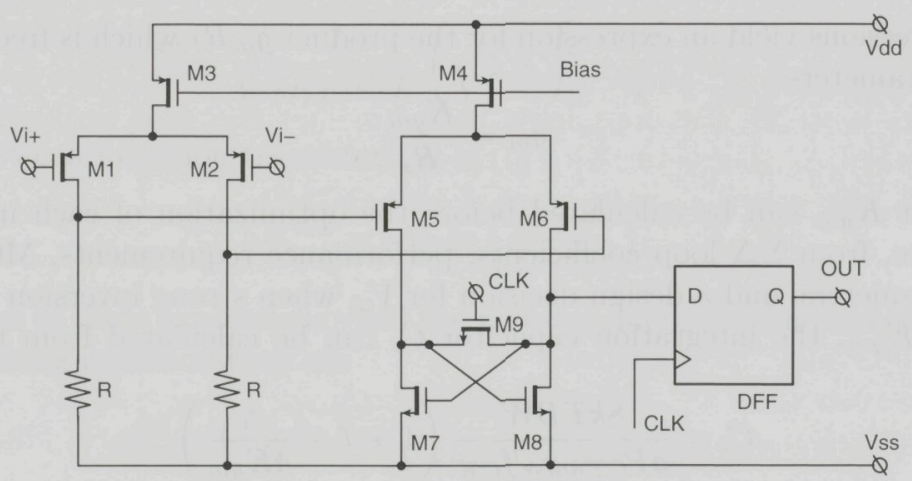

Figure 4.11: Fast comparator for CT $\Sigma \Delta$ ADCS 
with the transconductance transistors M5-M6 supplying the current for the latch $M 7-M 8$. The input stage, built with $M 1-M 2$ having resistive loads, is a preamplifier which adds extra delay so it is only used if kickback or offset must be minimized [9]. The latch, built with NMOS transistors for fast switching, is held in metastable condition by the reset switch $M 9$ for half the clock period, and then is allowed to make a decision which sets the state of the flip-flop $D F F$. The flip-flip is clocked by a delayed version of $C L K$ to obtain a signal-independent excess loop delay.

\subsection{Digital-to-Analog Converters}

As it has been shown with the integrator topologies, there are more options in implementing the DACs either with resistors or current sources. In all cases, one important issue of DAC design for $\Sigma \Delta$ ADCs is the ability to operate the DAC in return-to-zero (RTZ) mode, to reduce in-band quantization noise leakage due to inter-symbol modulation especially when high DR is targeted. An issue of importance only in multi-bit DACs is the DAC linearity which, for a DAC built from identical DAC cells, translates in a matching constraint for the constituent cells. In current-mode CT DACs the matching can be improved in an analog manner (as opposed to resistive- or capacitive-ladder switching available for $\mathrm{SC}$ or RC multibit DACs) by calibration. These two issues are covered in the present Section.

\subsubsection{Return-to-Zero DACs}

For the resistive DAC in Fig. 4.3, a RTZ switch is added as shown in Fig. 4.12. The switches coupling the two resistors to Vreft and Vref-are only active (following the quantizer decision $B O$ ) when the $R T Z$ signal is inactive. When $R T Z$ is active, they disconnect the DAC resistors from the reference voltages, and the switch controlled by $R T Z$ is closed, connecting the two ends of the DAC resistors. The advantage of this method is its simplicity, paid for with a RTZphase residual integration current produced on the two Rdac resistors by the residual voltage at the input of the opamp. This residual current can be reduced

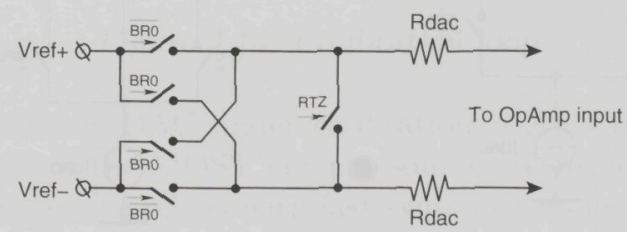

Figure 4.12: $R T Z$ switching of resistive $D A C$ 


\section{Continuous-Time Circuit Design}

by implementing the RTZ switch with a large ON-resistance, maximizing it in a trade-off with the increased gate-source and -drain capacitances and increased charge injection.

For the current-mode DACs, a simple switching logic disconnecting the DAC current sources from the integrator (see Figs. 4.4, 4.5) is not the best option for RTZ implementation. To avoid the saturation of the DAC current sources during the RTZ phase, the current-steering DAC in Fig. 4.13 (left) is equipped with an additional RTZ switch which connects the current source Idac to a resistive load during the RTZ phase. For the rest of the time, the switches controlled by the quantizer decision are active to connect the current source to either one of the opamp input nodes. Taking advantage of the existence of two current sources in the pseudo-differential, current-mode DAC, its RTZ version in Fig. 4.13 (right) only employs one RTZ switch to connect the two sources drainto-drain during the RTZ phase, keeping all the quantizer-controlled switches in off-state. For identical source/sink currents, there is no saturation whatsoever. In practice, the Idac+ and Idac-only match within 1-2\%, the difference being handled in the MOS current sources by $g_{d s}$ conduction. As long as the difference between Idact and Idac- is not very large it can be therefore accommodated with minimal $V_{d s}$ changes. If the difference is large, two RTZ switches can be used to switch each of Idac+ and Idac- to resistors connected to ground and supply, respectively, similar with the RTZ control of the current-steering DAC, at the price of doubled power consumption during the RTZ phase. Outside the RTZ phase, the four quantizer-controlled switches are used to connect the current sources to the opamp input nodes.
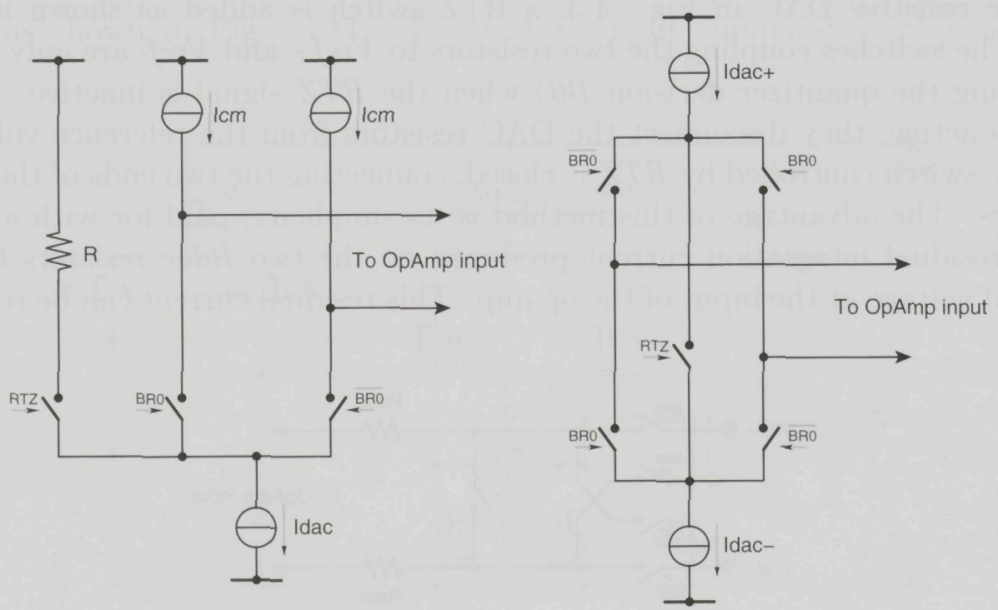

Figure 4.13: RTZ switching of current-mode DACs 


\subsubsection{Calibrated Multibit DACs}

In $\Sigma \Delta$ ADCs, the number of DAC bits is reduced due to oversampling and quantization noise shaping, hence the multi-bit DACs are normally designed as a collection of unitary DAC cells in $\Sigma \Delta$ ADCs. Since multibit DACs are built from multiple cells, matching of these cells is the main concern since it can degrade the conversion linearity. Calibration can be employed to correct the mismatch of DAC cells with lower power consumption than a complicated mismatch-shaping circuit as shown in [10] and with less spurious behavior than dynamic element matching [11]. Calibration is more flexible, it can be adapted to many DAC designs and its performance can be tweaked by trading accuracy for time and power. It is difficult to apply to passive DACs like resistor-strings or switched-capacitor, but it fits the current-mode DACs perfectly.

A background calibration loop (Fig. 4.14) contains a circuit for comparison between the analog output DAC_OUT of the DAC cell under calibration and a reference signal $R E F$. In the case depicted here, the comparison between the analog signals is performed by the Calibration $A D C$ which can be either a simple comparator (1-bit ADC) or a multibit ADC. The result of the comparison is supplied in digital domain to a Calibration $D A C$ which modifies the value of the $D A C$ cell under calibration by controlling the $C A L_{-} F B$ analog signal.

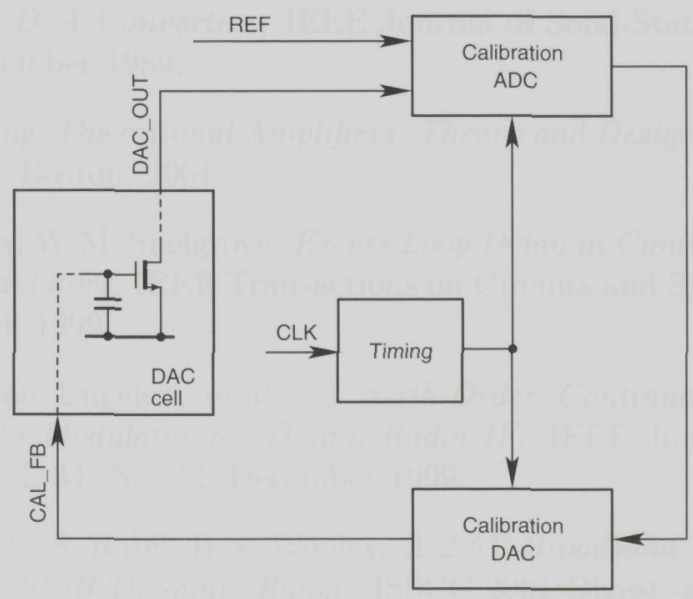

Figure 4.14: Calibration loop

For the current-mode DAC under calibration, $C A L_{-} F B$ is used to charge the gate capacitance of the MOST current source. Considering inherent leakage currents and charge couplings with fast-switching nodes, there is a minimal gate capacitance required to guarantee the DAC cell output variation from one calibration slot to another. Hence, for extended memory effect, the gate capaci- 


\section{Continuous-Time Circuit Design}

tance can be enhanced by an additional analog memory capacitor. The Timing block generates the synchronization signals for the calibration ADC and DAC. 


\section{References}

[1] M. Ortmanns, F. Gerfers, Y. Manoli, On the Synthesis of Cascaded Continuous-Time $\Sigma \Delta$ Modulators IEEE International Symposium on Circuits and Systems, 2001, Vol. 5.

[2] R. Mittal, D.J. Allstot, Low-Power High-Speed Continuous-Time $\Sigma \Delta$ Modulators IEEE International Symposium on Circuits and Systems, 1995, Vol. 1.

[3] E.J. van der Zwan, E.C. Dijkmans, A 0.2- $m W$ CMOS $\Sigma \Delta$ modulator for Speech Coding with 80 dB Dynamic Range, IEEE Journal of Solid-State Circuits, Vol. 31, pp. 1873, December 1996.

[4] L. Breems, J.H. Huijsing, Continuous-Time Sigma-Delta Modulation for $A / D$ Conversion in Radio Receivers, Kluwer Academic Publishers, Boston, 2001 .

[5] A.L. Coban, A Low-Voltage High-Resolution Delta-Sigma Modulator, PhD Thesis, Georgia Institute of Technology, May 1998.

[6] D.W.J. Groeneveld, et.al., A Self-Calibrated Technique for Monolithic HighResolution D/A Converters, IEEE Journal of Solid-State Circuits, Vol. 24, No. 6, December 1989.

[7] J.H. Huijsing, Operational Amplifiers. Theory and Design, Kluwer Academic Publishers, Boston, 2001.

[8] J.A. Cherry, W.M. Snelgrove, Excess Loop Delay in Continuous-Time DeltaSigma Modulators, IEEE Transactions on Circuits and Systems - II, Vol. 46, No. 4, April 1999.

[9] J.A.E.P. van Engelen, et.al. A Sixth-Order Continuous-Time Bandpass Sigma-Delta Modulator for Digital Radio IF, IEEE Journal of Solid-State Circuits, Vol. 34, No. 12, December 1999.

[10] K. Vleugels, S. Rabii, B.A. Wooley, A $2.5 \mathrm{~V}$ Broadband Multi-Bit $\Sigma \Delta$ Modulator with 95dB Dynamic Range, ISSCC 2001 Digest of Technical Papers, Feb. 2001.

[11] E. Fogleman, I. Galton, H. Jensen, A Dynamic Element Matching Technique for Reduced-Distortion Multibit Quantization in Delta-Sigma ADCs, IEEE International Symposium on Circuits and Systems, 1999, Vol. 2. 
REFERENCES

102 Systematic Design of $\Sigma \Delta$ ADCs 


\section{5}

\section{Computer Aided Design of Sigma-Delta ADCs}

Since no precise analytical model is available for $\Sigma \Delta$ ADCs in general, there is need to simulate different possible solutions before deciding which one has the potential to be the optimal one. Even if a local optimum is targeted, for example if the constraint is to use a fourth order design, the lack of a good analytical model undermines the use of gradient-based methods. This Chapter presents a systematic procedure to exhaustively explore the design space of $\Sigma \Delta$ ADCs, by automatically designing and evaluating a large number of candidate solutions based on behavioral simulations. Section 5.1 presents the first level of abstraction used in this procedure, the filter level, where the linear model is used (with all its limitations) to reduce the number of possible solutions. Section 5.2 presents the second abstraction level, the architecture level, where the $\Sigma \Delta \mathrm{ADC}$ loops are actually designed and simulated to measure their performance. Two design examples are shown in Section 5.3.

\subsection{Filter-Level Design}

A fast design space exploration is performed at filter-level, using a linearized model of the $\Sigma \Delta$ ADC. The goal of this search is to quickly evaluate all solutions in the design space and select only candidates that can attain the target dynamic range (DR). The search algorithm is shown in Fig. 5.1. The entire $\Sigma \Delta \mathrm{ADC}$ design space is split in two sub-spaces, the topology design sub-space defined by the ORDER, number of cascaded LOOPS and number of BITS, and the parameter design sub-space defined by the oversampling ratio $O S R$ and peak NTF (noise transfer function) magnitude. All these dimensions of the search space are browsed using constant stepping, linearly in topology space 


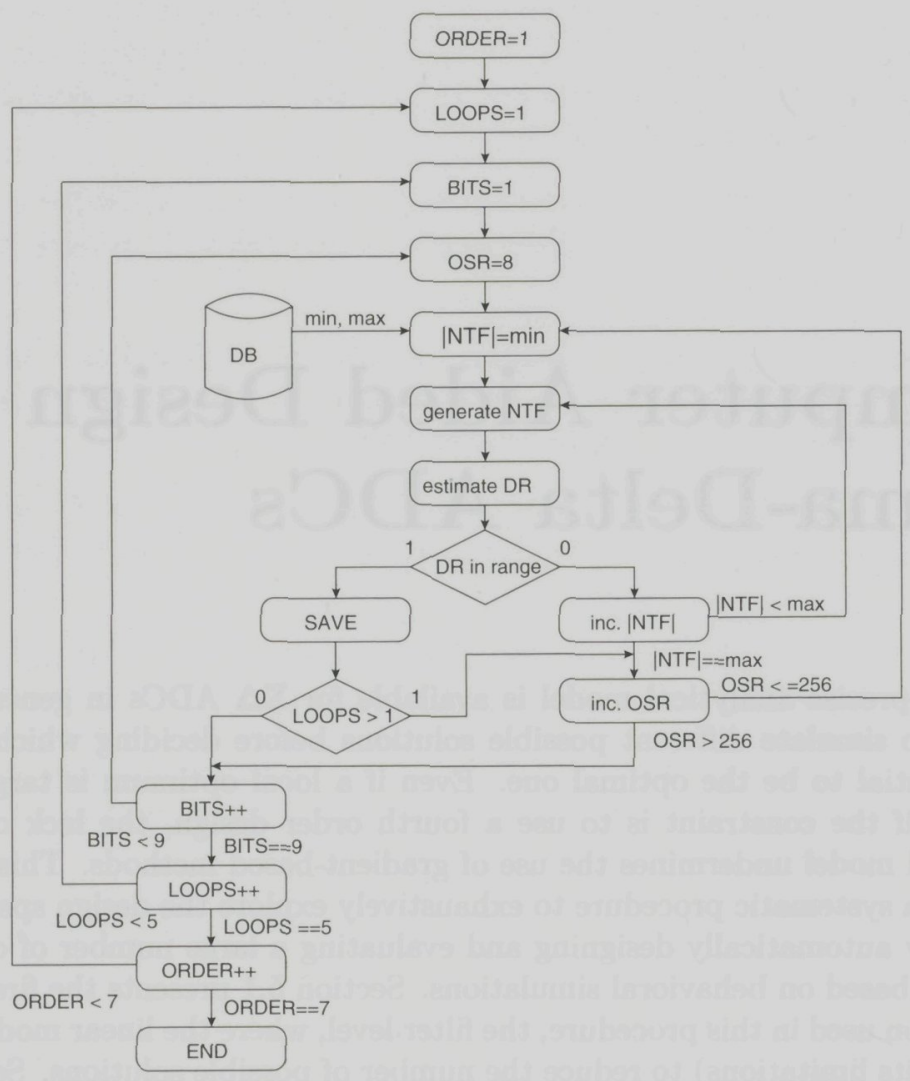

Figure 5.1: Filter-level exploration algorithm

and exponentially in parameter space.

At each step a set of two filter transfer functions are generated, the NTF and the signal transfer function (STF). As a provision for direct sensor interfacing, it is possible to set the STF gain to values different from unity. Filter generation can also optimize in-band zeros for the NTF (to reduce total in-band noise power [1]) and off-band zeros for the STF (to reduce in-band gain ripple and increase off-band rejection). After the two filter transfer functions are generated the in-band noise power is calculated to estimate the DR. A test is applied with two thresholds derived from the target DR (in $\mathrm{dB}$ )

$$
D R_{\text {target }}+D R_{\text {lower }} \leq D R \leq D R_{\text {target }}+D R_{\text {upper }}
$$




\subsection{Filter-Level Design}

The lower limit tests if, after the circuit (white) noise is added, the converter still reaches the target DR. The upper limit is set to reject power-hungry solutions which offer more quantization-noise DR than actually needed. If the test is passed, the solution is saved in a database.

For single-loop solutions the algorithm stops when a combination of minimal $O S R$ and NTF is found. For cascaded designs however, because significant architectural details are not available at the filter level, the algorithm continues searching for solutions even after finding the first valid one. This insures that no valid solution is prematurely rejected.

The limits within which $N T F$ is browsed are decided based on a database stored on disk containing, for each set of (ORDER, BITS), the value of the overloading level. This database is built by applying the entire design procedure explained in this chapter (i.e., filter-level and architecture-level design) to the entire topology design sub-space with the LOOPS constrained to 1 because overloading level of cascaded solution is ultimately decided by the first loop. In the parameter sub-space, $N T F$ is sampled in a wide range while $O S R$ is set to 64 , because $O S R$ does not affect the overloading level. The use of the NTF database results can drastically reduce run-time because the solutions considered as possible candidates are actually guaranteed to yield stable $\Sigma \Delta$ loops.

\subsubsection{Design of Stable Discrete-Time NTF}

The design of NTF characteristics suitable for use as $\Sigma \Delta$ loop filters is performed by generating a discrete-time (DT) NTF with Chebyshev poles that yields the peak NTF magnitude required at each NTF step in the parameter design subspace. This is done by adjusting the cut-off frequency of a high-pass filter generated with the equations outlined in the following sub-Section. The stability of the designed NTF is guaranteed by its peak magnitude lying inside the range extracted from the NTF database, depending on the $\Sigma \Delta$ loop ORDER and quantizer BITS.

\section{DT NTF with Chebyshev Poles}

The design of candidate NTF filter consists of calculating a set of Chebyshev poles for a low-pass filter which transformed to a high-pass equivalent [3] yields a causal filter. Design requirements are the cut-off frequency and passband ripple. The choice of NTF filter family (Chebyshev, Butterworth or elliptic) does not affect the overloading level of the $\Sigma \Delta$ ADC for the same filter aggressiveness. However, high NTF passband ripple can decrease the overloading level. The low-ripple Butterworth family is convenient from this point of view but does not tolerate off-DC zeros which sometimes can be employed for increased inband quantization noise rejection [1]. Therefore, Chebyshev filters are used 
in the CAD software, as they offer controlled passband ripple and keep their characteristics with off-DC zeros.

For a continuous-time (CT) filter, the amplitude characteristic $|H(\omega)|$ of a low-pass Chebyshev filter of order $n$ and having a cut-off frequency $\omega_{c}$ is

$$
|H(\omega)|=\frac{1}{\left(1+\epsilon^{2} C_{n}^{2}\left(\frac{\omega}{\omega_{c}}\right)\right)^{1 / 2}}
$$

where $\omega$ is the CT frequency, $C_{n}$ is the Chebyshev polynomial of order $n$ and $\epsilon$ is related to the passband ripple $\delta$ by

$$
\delta=1-\left(1+\epsilon^{2}\right)^{-1 / 2}
$$

Chebyshev polynomials are generated recursively by

$$
\begin{aligned}
& C_{0}(x)=1 \\
& C_{1}(x)=x \\
& C_{n}(x)=2 x C_{n-1}(x)-C_{n-1}(x)
\end{aligned}
$$

By using a bilinear transform [3] the CT filter is transformed to a DT correspondent

$$
|H(\Omega)|=\frac{1}{\left(1+\epsilon^{2} C_{n}^{2}\left(\frac{\tan (\Omega / 2)}{\tan \left(\Omega_{c} / 2\right)}\right)\right)^{1 / 2}}
$$

with $\Omega$ being the DT frequency, and $\Omega_{c}$ being the cutoff frequency in DT domain. From this form of the transfer function, the poles result with their real and imaginary parts

$$
\begin{aligned}
& \Re\left(P_{i}\right)=2 \frac{1-A \tan \left(\Omega_{c} / 2\right) \cos (\Phi)}{\left(1-A \tan \left(\Omega_{c} / 2\right) \cos (\Phi)\right)^{2}+B^{2} \tan \left(\Omega_{c} / 2\right)^{2} \sin (\Phi)^{2}}-1 \\
& \Im\left(P_{i}\right)=2 B \tan \left(\Omega_{c} / 2\right) \sin (\Phi)
\end{aligned}
$$

as functions of

$$
\begin{aligned}
& A=\frac{1}{2}(\sqrt[n]{C}-\sqrt[-n]{C}) \\
& B=\frac{1}{2}(\sqrt[n]{C}+\sqrt[-n]{C}) \\
& C=\left(1+\epsilon^{-1}+\epsilon^{-2}\right)^{1 / 2}
\end{aligned}
$$

The frequency $\Phi$ is a discrete variable which is a function of pole index $i$

$$
\Phi= \begin{cases}\frac{i \pi}{n} & \text { if } n \text { is odd } \\ \frac{(2 i+1) \pi}{2 n} & \text { if } n \text { is an even }\end{cases}
$$


For the low-pass filter there is a zero at $f_{s} / 2(z=-1)$ of order $n$. Transforming the low-pass into a high-pass simply means changing the sign of real parts of all poles. The zeros are also inverted to move to DC for the high-pass filter. If $n$ is odd, the real pole takes the value

$$
P_{0}=\frac{2}{1+\operatorname{Atan}\left(\Omega_{c} / 2\right)}-1
$$

\section{NTF Database}

The one performance parameter of $\Sigma \Delta$ ADCs that cannot be predicted accurately by linear modeling is the overloading level (OVL) [2]. In the algorithm presented here, the overloading levels for each single-loop $\Sigma \Delta \mathrm{ADC}$ in the entire range of loop orders and quantizer bits are computed once from time-domain simulations and stored in a database. The peak magnitude of NTF is varied in a range from $1.0(0 \mathrm{~dB})$ to $16.0(24 \mathrm{~dB})$. Each single-loop $\Sigma \Delta \mathrm{ADC}$ is designed and optimized in a range of input signal values for each NTF peak magnitude. The overloading level is detected as the input signal which causes integrator clipping.

Fig. 5.2 shows the entry in the peak NTF database for the single-bit, fourthorder $\Sigma \Delta$ ADC. It shows the variation of overloading level $O V L$, peak output of

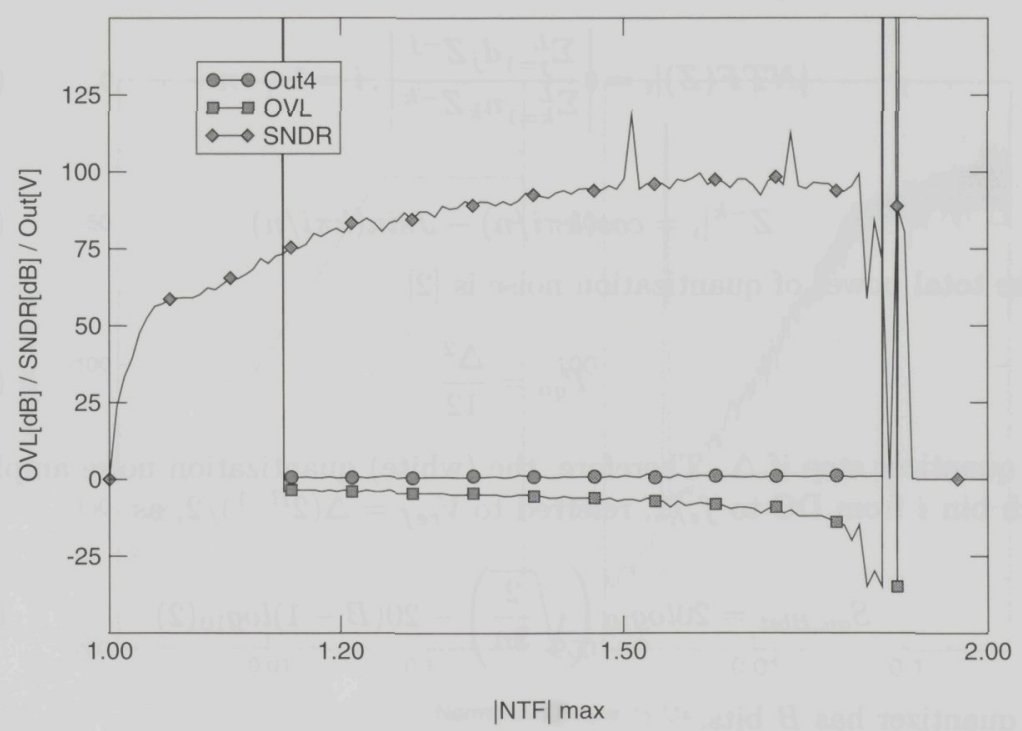

Figure 5.2: Peak NTF magnitude database entry example (ORDER=4, $B I T S=1, V_{\text {clip }} / V_{\text {Ref }}=1$ ) 


\section{Computer Aided Design of Sigma-Delta ADCs}

the last integrator Out4 and the peak signal-to-noise+distortion SNDR in the NTF range where the loop can be stabilized. The decision that the loop can be stabilized is taken by comparing the values of integrator coefficients against a bottom value. If all integrator coefficients are larger than the bottom value and integrator outputs are smaller than the $V_{\text {clip }}$ value, the loop can be designed stable and there exists a dB-negative overloading level (see Chapter 2).

Because of this tight relationship between the $O V L$ value and the ratio of the clipping voltage over reference voltage, the database has to be generated for each different ratio of $V_{\text {clip }} / V_{\text {Ref }}$. This takes about 24 hours on a $1 \mathrm{GHz}$ computer and the same database can be used for any target DR (SNDR) design as long as the $V_{\text {clip }} / V_{\text {Ref }}$ ratio does not change.

\subsubsection{Dynamic Range Estimation}

A fast yet accurate method to estimate the DR is used. It overcomes the drawbacks of both time-domain simulations, which are slow, and classical DR estimation using a formula which is inaccurate especially for high-order $\Sigma \Delta$ loops [2]:

$$
D R=\frac{3}{2} \frac{2 L+1}{\pi^{2 L}} O S R^{2 L+1}\left(2^{B}-1\right)^{2}
$$

First the magnitude of NTF is calculated in $n$ points equally distributed from DC to $f_{s} / 2$

$$
|N T F(Z)|_{i}=\left|\frac{\sum_{j=1}^{L} d_{j} Z^{-j}}{\sum_{k=1}^{L} n_{k} Z^{-k}}\right|, i=1 \cdots n
$$

using

$$
\left.Z^{-k}\right|_{i}=\cos (k \pi i / n)-J \sin (k \pi i / n)
$$

The total power of quantization noise is [2]

$$
P_{q n}=\frac{\Delta^{2}}{12}
$$

if the quantizer step if $\Delta$. Therefore, the (white) quantization noise amplitude in each bin $i$ from DC to $f_{s} / 2$, referred to $V_{\text {ref }}=\Delta\left(2^{B-1}\right) / 2$, as

$$
S_{q n, B b i t}=20 \log _{10}\left(\sqrt{\frac{2}{3 n}}\right)-20(B-1) \log _{10}(2)
$$

if the quantizer has $B$ bits.

The value of the NTF magnitude, given by Eq. (5.11) expressed in dB, is added to the value of $S_{q n, B b i t}$ and a curve showing the quantization noise amplitude in each bin is drawn. Its integral in the band of interest yields the 
estimated DR value. Fig. 5.3 shows the estimated (left) and time-domain simulated (right) spectrum of the output of a fourth-order, one-bit, single-loop $\Sigma \Delta$ ADC. The two graphs show the good accuracy of this estimation method for high-order loops.

The DR of the fourth-order, single-loop, single-bit $\Sigma \Delta$ ADC operated at 64 times OSR is estimated to be $102 \mathrm{~dB}$, which is only $3 \mathrm{~dB}$ different from the time-domain simulated result of $105 \mathrm{~dB}$. As a comparison, the formula in Eq. (5.10) yields $134 \mathrm{~dB}$, due to the idealized NTF expression used to derive it [2]. An extended comparison between the classical formula and the polynomial estimation method applied to single-loop $\Sigma \Delta$ ADCs is shown in Fig. 5.4. The set of analyzed solutions is listed on the x-axis as sets of (ORDER, OSR, BITS). The full line shows the error of the polynomial estimation method as the difference between estimated value and time-domain simulation results. The error is always less than $4 \mathrm{~dB}$, as opposed to the error yielded by calculating the DR using the classical formula (dotted line on the graph) which can be comparable to the target DR itself.

The polynomial estimation method also works better than the calculation for cascaded loops. Fig. 5.5 shows the errors yielded by the two methods for a class of fifth order $\Sigma \Delta$ ADCs with two to four loops in cascade and the number of bits in the last loop varying from two to seven. Only the number of bits in the last loop is considered for the quantizer because it is the parameter that has to be used as B in Equation 5.10.

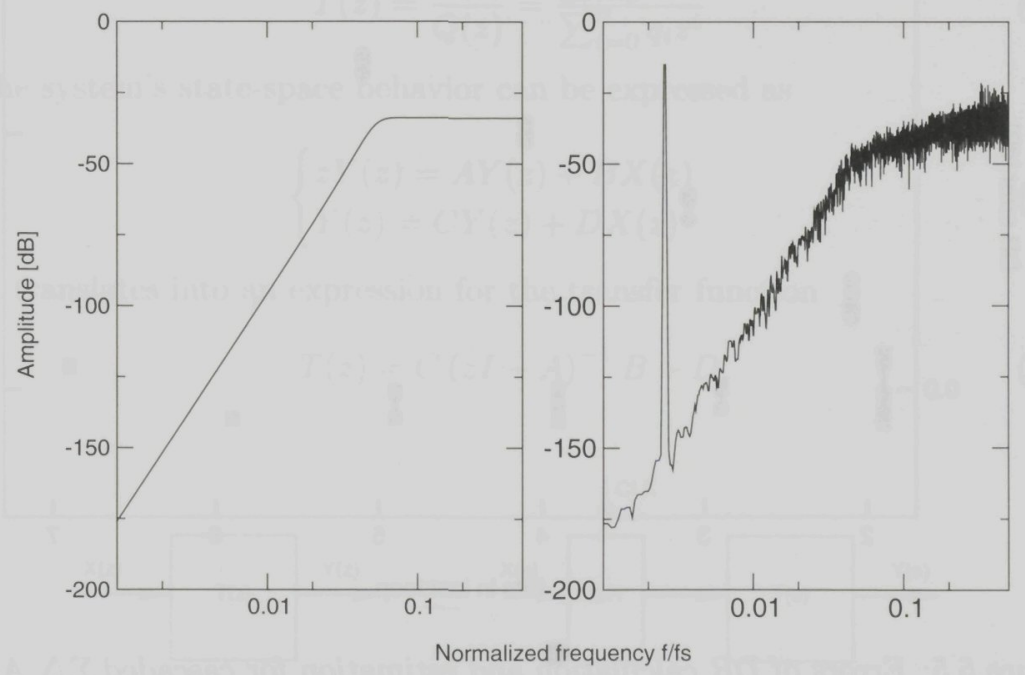

Figure 5.3: Estimated (left) and time-domain simulated (right) quantization noise spectrum for $\mathrm{ORDER}=4, \mathrm{BITS}=1, \mathrm{LOOPS}=1$ 
Computer Aided Design of Sigma-Delta ADCs

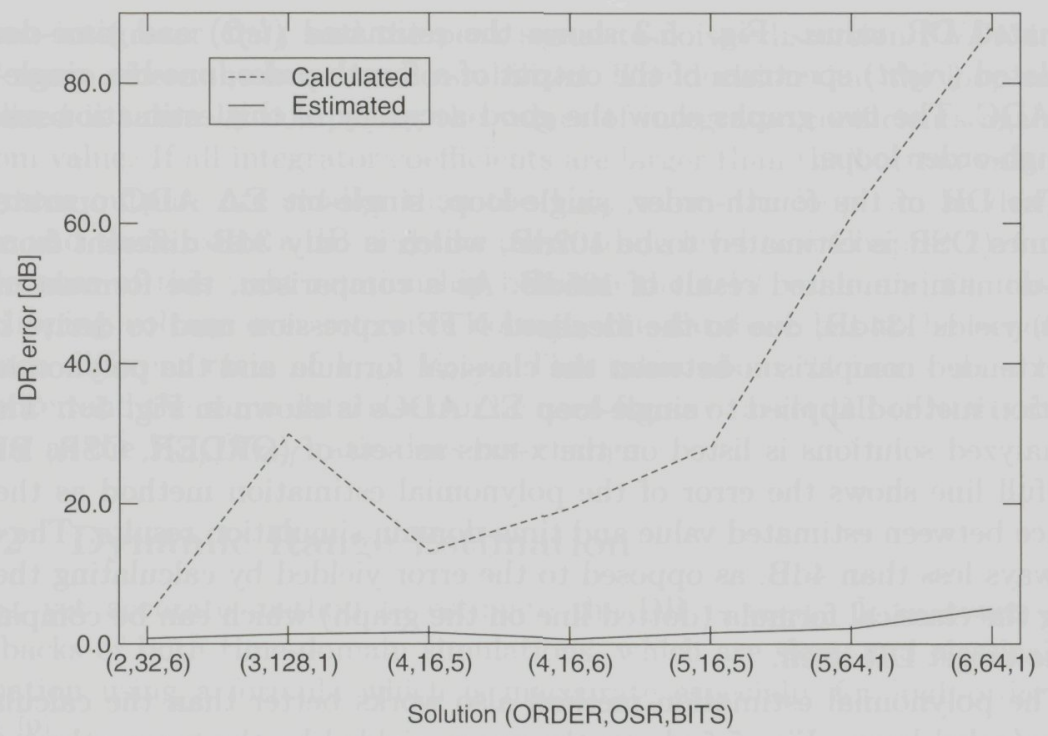

Figure 5.4: Errors of DR calculation and estimation for single-loop $\Sigma \Delta A D C s$

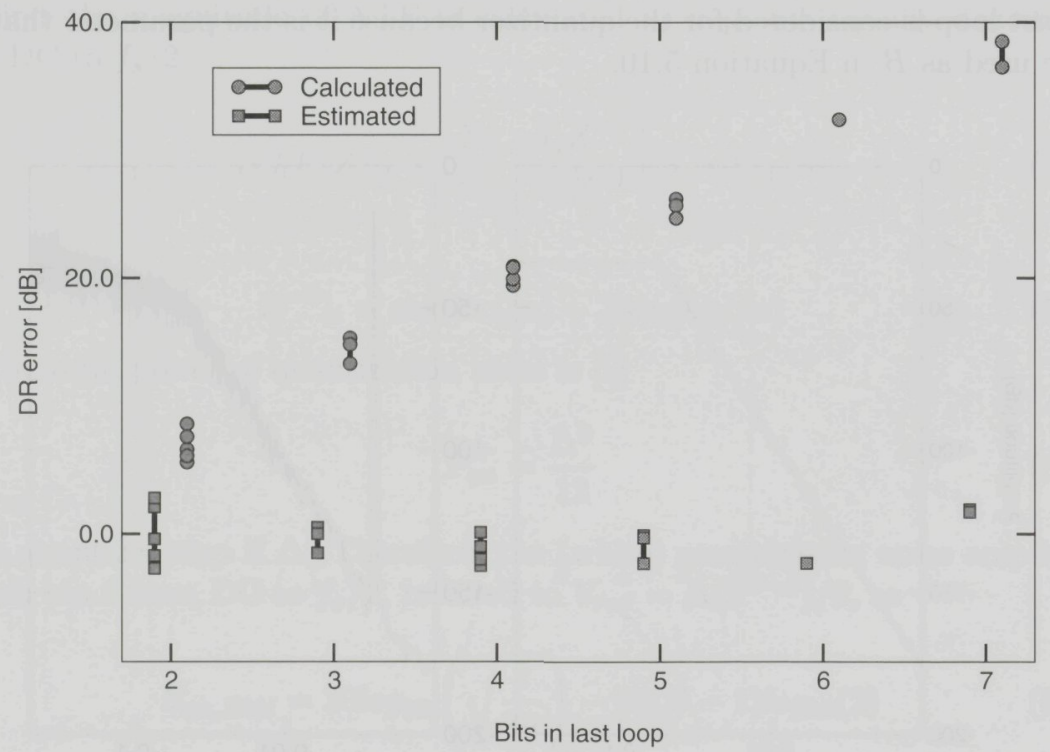

Figure 5.5: Errors of DR calculation and estimation for cascaded $\Sigma \Delta A D C s$ 


\subsection{Filter-Level Design}

\subsubsection{Mapping DT TF to CT TF}

The design of CT transfer functions (both NTF and STF) is done in two stages. Firstly, a DT transfer function pair is calculated with Chebyshev poles using the equations explained in sub-Section 5.1.1. Secondly, the generated pair of DT transfer functions is mapped to CT equivalents to be used in CT $\Sigma \Delta$ modulators. Both NTF and STF undergo the same mapping. This two-stage design procedure for CT modulators allows the same NTF aggressiveness metric to be used for both DT and CT design, namely the peak NTF magnitude.

A DT system and a CT one are shown in Fig. 5.6. The two systems are equivalent if, under the assumption that the two input signals are equivalent at discrete time moments,

$$
X(n)=\left.X(t)\right|_{t=n T}, T=\frac{1}{f_{s}}
$$

the two outputs are equivalent

$$
Y(n)=\left.Y(t)\right|_{t=n T}
$$

The mapping from DT to CT is therefore done assuming a zero-order hold behavior for the sampler in front of the CT system. Because both NTF and STF are rational transfer functions, the DT transfer function $\mathrm{T}(\mathrm{z})$ can be generally written as

$$
T(z)=\frac{R(z)}{Q(z)}=\frac{\sum_{i=0}^{n} r_{i} z^{i}}{\sum_{i=0}^{n} q_{i} z^{i}}
$$

and the system's state-space behavior can be expressed as

$$
\left\{\begin{array}{l}
z Y(z)=A Y(z)+B X(z) \\
Y(z)=C Y(z)+D X(z)
\end{array}\right.
$$

which translates into an expression for the transfer function

$$
T(z)=C(z I-A)^{-1} B+D
$$

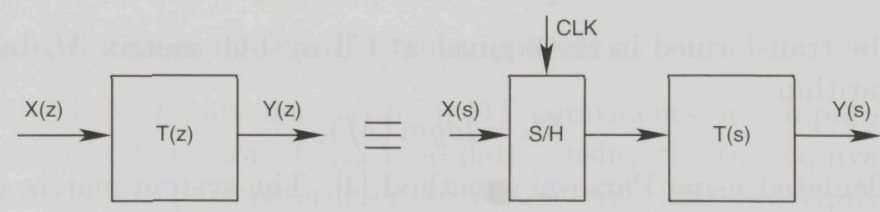

Figure 5.6: Equivalent DT and CT systems 
with I being the identity matrix eye $(n)$ (contains ones on main diagonal, the rest is filled with zeros). To express the system in a matrix form convenient for DT to CT mapping, the first step is to subtract the constant term D from T(z)

$$
D=T(\text { inf })=\frac{r_{n}}{q_{n}},
$$

building $T_{1}(z)$ as

$$
T_{1}(z)=T(z)-D=\frac{R(z)-D Q(z)}{Q(z)}=\frac{R_{1}(z)}{Q(z)}
$$

with

$$
R_{1}(z)=\sum_{i=0}^{n} r_{1_{i}} z^{i}
$$

The matrices $A, B$ and $C$ are now expressed as

$$
\begin{aligned}
& A=\left[\begin{array}{cc}
-q_{n-1} & \\
-q_{n-2} & \\
\vdots & \text { eye }(n-1) \\
-q_{1} & \\
-q_{0} & 0 \cdots 0
\end{array}\right] \\
& B=\left[\begin{array}{c}
r_{1_{n-1}} \\
r_{1_{n-2}} \\
\vdots \\
r_{1_{1}} \\
r_{1_{0}}
\end{array}\right] \\
& C=\left[\begin{array}{llll}
1 & 0 & \cdots & 0
\end{array}\right]
\end{aligned}
$$

and are used to build the system matrix $\mathrm{M}$

$$
M=\left[\begin{array}{cccc} 
& A & & B \\
0 & \cdots & 0 & 1
\end{array}\right]
$$

which can be transformed in the equivalent CT system matrix $M_{C}$ by a matrix natural logarithm

$$
M_{c}=\log m(M)
$$

which is calculated using Parseval's method [4]. The system matrix $M_{c}$ results in a form similar to $\mathrm{M}$

$$
M_{c}=\left[\begin{array}{cccc} 
& A_{c} & & B_{c} \\
0 & \cdots & 0 & 0
\end{array}\right]
$$


where the $A_{c}$ and $B_{c}$ matrices can be identified from. The $C_{c}$ and $D_{c}$ matrices are identical with their DT correspondents $\mathrm{C}$ and D and the entire CT system equivalent to the DT T(z) can be now expressed as

$$
T_{c}(s)=C_{c}\left(s I-A_{c}\right)^{-1} B_{c}+D_{c}
$$

From this state-space equation the poles and zeros of $T_{c}$ are extracted. The poles are the eigenvalues of $A_{c}$

$$
P\left(T_{c}(s)\right)=\operatorname{eig}\left(A_{c}\right)
$$

and the zeros are calculated in a more laborious way as the eigenvalues of the matrix

$$
A_{i n v}=A_{c}-\frac{B_{c} C_{c}}{D_{c}},
$$

therefore

$$
Z\left(T_{c}(s)\right)=\operatorname{eig}\left(A_{\text {inv }}\right)
$$

By noting $\operatorname{poly}(X)$ the polynomial built with the elements in vector $\mathrm{X}$ as roots, the CT transfer function $T_{c}$ results

$$
T_{c}=\frac{D_{c} p o l y\left(Z\left(T_{c}(s)\right)\right)}{\operatorname{poly}\left(P\left(T_{c}(s)\right)\right)}
$$

For STF, there are cases when $D_{c}$ is zero. In these cases, a non-zero offset is applied to $D_{c}$

$$
D_{c}=D_{o f f s e t}
$$

and $T_{c}$ results

$$
T_{c}=\frac{D_{\text {offset }}\left(\text { poly }\left(Z\left(T_{c}(s)\right)\right)-\text { poly }\left(P\left(T_{c}(s)\right)\right)\right)}{p o l y\left(P\left(T_{c}(s)\right)\right)}
$$

The equations above map the DT filters to CT filters with zero-order hold input signals [5]. For the NTF this means a non-return-to-zero DAC pulse is used. If the DAC pulse is not a $\mathrm{ZOH}$ signal (i.e., constant during one clock period) additional transforms have to be applied to the $A_{c}$ and $B_{c}$ matrices [2].

\section{Comparison between DT and CT loop filters}

Because the CT NTFs are derived from DT equivalents, a comparison can be made between the behavior of $\Sigma \Delta$ modulators using the two equivalent NTFs as loop filters. The parameter used to characterize the two equivalent loop filters is the peak amplitude of the DT NTF. The comparison shows that, even if the loop filters are equivalent, the behavior of the time-domain simulated modulators depend on the nature of the loop filter. 
The OVLs of equivalent, fourth-order CT and DT modulators with 1-bit quantizers are plotted in Fig. 5.7. On the $X$-axis, the parameter NTFmax denoting the peak magnitude of the DT NTF is stepped exponentially. This graph is obtained by generating the entire range of DT NTFs characterized by their NTFmax, building the equivalent CT NTF, and designing the two equivalent DT and CT modulators with optimized loop coefficients on identical topologies. Time-domain simulations are performed with the DT and CT equivalents and their OVLs are measured. The range of NTFmax where the OVL takes negative $\mathrm{dB}$ values is the range of NTFs that yield a stable modulator. It can be seen that there is a minimal and maximal peak NTF that can be used to design a stable modulator. This shows that there is a minimal and a maximal amount of high-frequency quantization noise power that has to re-circulate inside the loop to keep it stable. Also, the CT designs have a lower usable range (from 1.1 to 1.55 on the $X$-axis) compared to the DT correspondents, which means CT designs cannot make use of highly-aggressive NTFs. This translates into a lower limit of attainable DR for the same OSR. Also, their peak SNDR is lower at the same NTF aggressiveness because their OVL is lower.

An explanation of this effect can be given based on the two other curves plotted in Fig. 5.7. They show the variation of the optimized coefficient of the first integrator a1 with NTF aggressiveness. For more aggressive NTFs, a1 increases, which in DT designs explains the decrease in OVL. Noting that

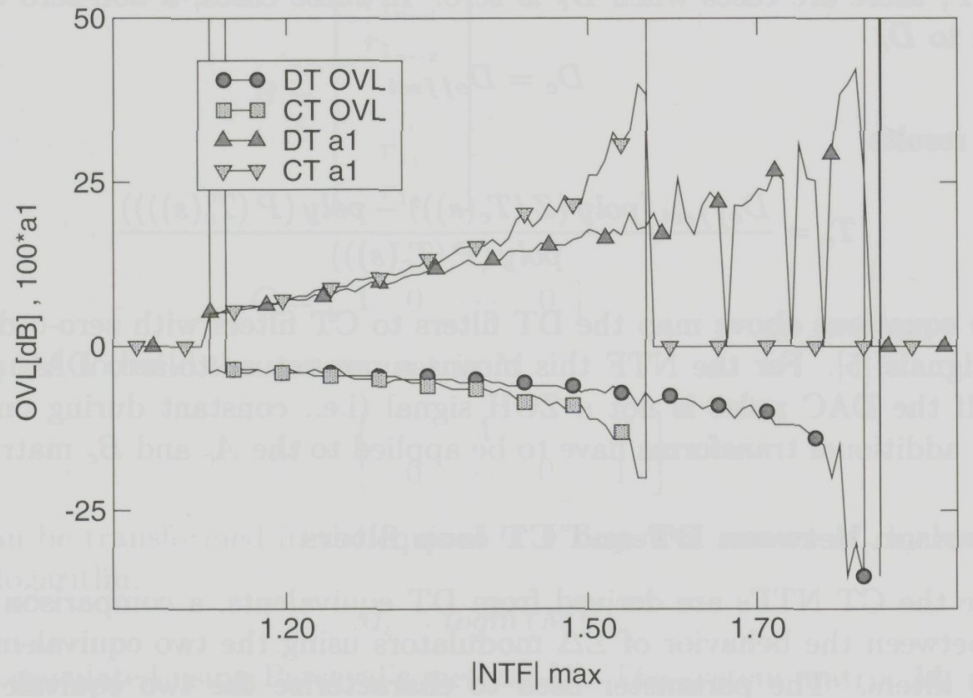

Figure 5.7: Peak NTF database entries for both DT and CT loops with ORDER $=4, B I T S=1$ 
the gradient of $\mathrm{CT}$ a1 is higher than that of DT a1, it becomes clear why CT designs tend to overload faster than DT equivalents and cannot reach higher levels of NTF aggressiveness.

\subsection{Architecture-Level Design}

\subsubsection{Design Space Exploration}

The architecture-level exploration algorithm evaluates the performance of the filter-level solutions mapped on a specific architecture, as shown in Fig. 5.8. If a single-loop solution is processed, an architecture is generated with the feedforward and feedback connectivity specified by the user. An initial set of coefficients are then calculated and simulations are performed with a wide range of input signal amplitudes to determine the OVL. Because the initial set of coefficients are not optimized they can cause premature overloading if integrator clipping to the supply rails is simulated. Therefore, for these simulations the supply voltage is considered to be 100 larger. Since the reference voltage remains the same, this simulation setup is equivalent to using no-clipping integrator outputs. Since the initial, un-optimized integrator coefficients are at most 10 times larger than the final, optimized coefficients, the 100 times increase in output range offer a sufficient margin.

The input signal applied at this stage is a pulse (a busy signal [2]) with a fundamental frequency three times lower than the signal bandwidth. With this fundamental, the main spectral component is present inside the conversion bandwidth along with the first two harmonics. Because more harmonics are present between DC and $f_{s} / 2$, the OVL is independent of input signal frequency (see OVL explanation in Chapter 2).

The next step is the coefficient optimization, which is performed at the OVL previously detected. This is performed by observing the peak output of each integrator and by correcting the associated loop coefficient accordingly. The SNDR and DR variations as a function of the input signal are then simulated using a sine input signal. The two curves are tested for performance and passing solutions are subject to a Monte-Carlo (MC) analysis to test the architecture's tolerance to coefficient mismatch. The solution is then saved for further processing.

For the cascaded $\Sigma \Delta$ ADCs the only architectural details predefined at filter level are the number of loops and the number of bits in the last loop. Therefore, the filter orders of individual cascaded loops are generated as one additional design space dimension named ORDERS in Fig. 5.8. Another new design space dimension is the number of BITS in the first loop of the cascade. For simplicity, the last $n-1$ loops in a cascade of $n$ loops have the same number of bits as the last one. 


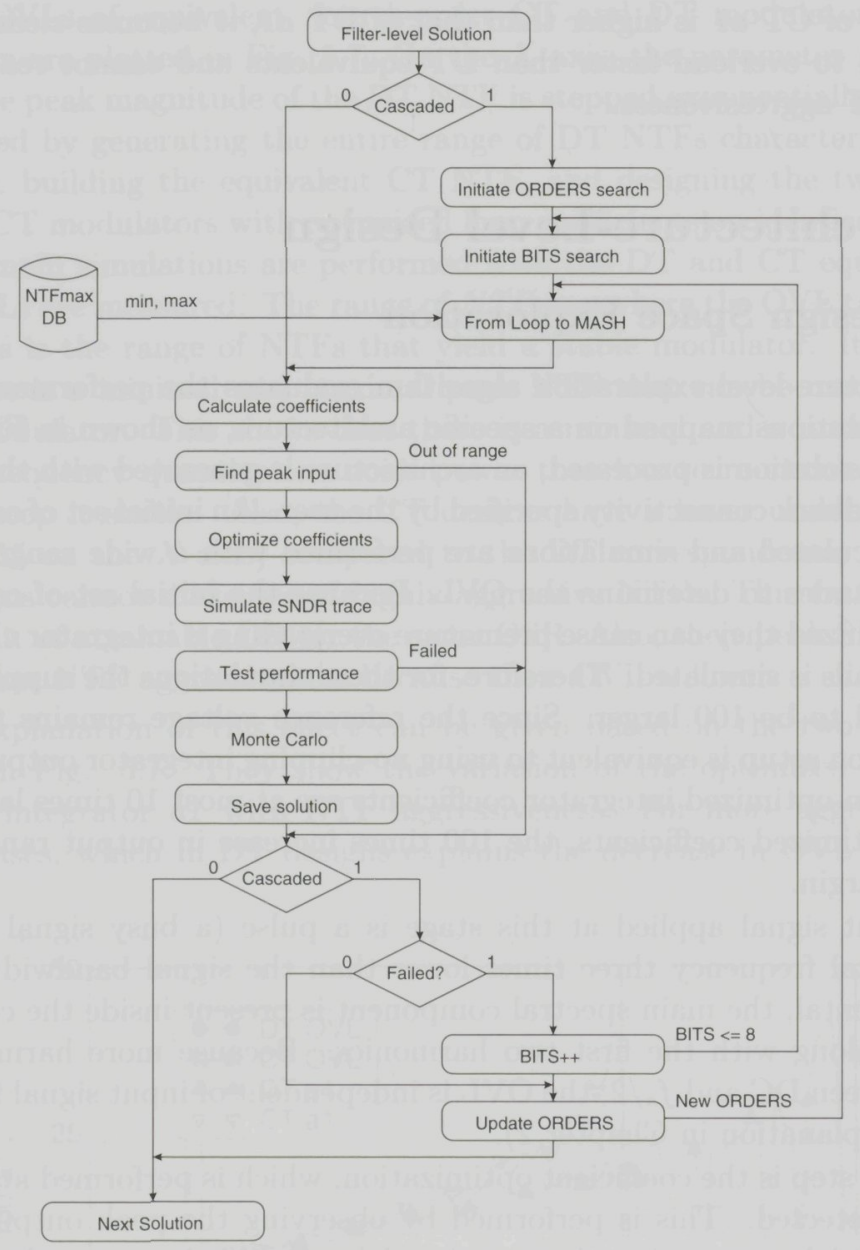

Figure 5.8: Architecture-level design space exploration

Each derivative of the input solution in the extended design space is analyzed as a possible solution. The architectural derivative is built starting from the parameters supplied in the filter-level solution, namely the peak NTF and the number of quantizer bits for the last loop in the cascade. The peak NTF in the filter-level solution is the product of peak NTF values in each loop

$$
|N T F|_{\text {filter-level }}=|N T F|_{1}|N T F|_{2} \cdots|N T F|_{n}
$$

and this equation can introduce an additional design space dimension set, the 


\subsection{Architecture-Level Design}

peak values of independent NTFs. However, the method of digitally re-combining individual outputs of cascaded loops with simple digital circuits introduces a design constraint in this respect. It has been observed that good quantization noise cancellation is achieved through digital filters in the shape of idealized, n-order NTF [6]

$$
\operatorname{NTF}(z)=\left(1-z^{-1}\right)^{n}
$$

only if the peak NTF value in each cascaded loop is the same. In other words, only identical NTFs can be used to design individual loops in the cascade, therefore the total peak NTF value is the product of $n$ identical values

$$
|N T F|_{\text {filter-level }}=|N T F|_{1}^{n}
$$

Based on this design constraint, the generation of a cascaded design architecture consists of finding the $|N T F|_{1}$ value that yield stable NTFs for all the loops in the cascade, no matter their individual order and quantizer bits. The individual orders and quantizer bits are set at this stage by the filter-level solution and the $O R D E R S$ and first quantizer BITS design parameters, so the range of common stable NTFs is found by performing a logical- $A N D$ between the stable ranges of each loop in the cascade, as extracted from the peak NTF database (see subSection 5.1.1). There are cases when the logical-AND yields a null range and the architectural derivative is then dropped.

Each individual loop in the cascade is then designed following the procedure described for single-loop solutions. The coefficients connecting the loops are derived from corresponding loop coefficients [6].

\subsubsection{Optimization of Loop Gains}

The goal of coefficient optimization is to map the two filters $\operatorname{NTF}(Z)$ and $\operatorname{STF}(Z)$ supplied with the filter-level solutions to a user-defined topology without causing unwanted integrator clipping. By correct optimization, clipping occurs when the input signal reaches the OVL of the loop and works as a stabilization mechanism. The integrator coefficients are limited based on integrator output observed in time-domain simulations and the rest of the coefficients are calculated based on $\operatorname{NTF}(Z)$ and $\operatorname{STF}(Z)$ expressions. The optimization is performed in two steps: coefficient calculation followed by coefficient scaling.

The explored solutions are associated with user-defined topologies in the form of square connectivity matrices. Single-loop solutions have one matrix associated while cascaded solutions have each loop in the cascade associated with one matrix. For the single-loop topology in Fig. 5.9 (a high-complexity topology used here only as an example), the connectivity matrix shown in Eq. 5.37 contains all the connections from the input node in column 1 , all the connections from the quantizer output in column 9 and all the connections to the quantizer input in row 8. Row 9 only contains the quantizer transfer function. All the 


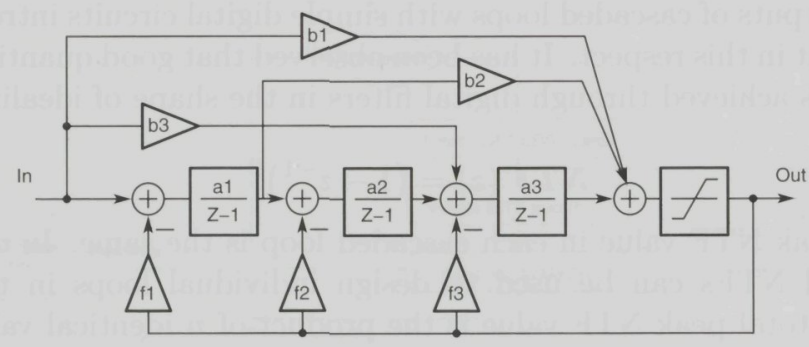

Figure 5.9: Architecture-level design space exploration

other connections are described around the integrator inputs and outputs, with column 2 being the first integrator input, column 3 the first integrator output and so on. The coefficients in the matrix are not simple numeric values, instead they are custom-defined data structures containing a type and a value along with a set of flags used for programming purposes.

$$
C M=\left[\begin{array}{ccccccccc}
0 & 0 & 0 & 0 & 0 & 0 & 0 & 0 & 0 \\
1 & 0 & 0 & 0 & 0 & 0 & 0 & 0 & f_{1} \\
0 & \frac{a_{1}}{Z-1} & 0 & 0 & 0 & 0 & 0 & 0 & 0 \\
0 & 0 & 1 & 0 & 0 & 0 & 0 & 0 & f_{2} \\
0 & 0 & 0 & \frac{a_{2}}{Z-1} & 0 & 0 & 0 & 0 & 0 \\
b_{3} & 0 & 0 & 0 & 1 & 0 & 0 & 0 & f_{3} \\
0 & 0 & 0 & 0 & 0 & \frac{a_{3}}{Z-1} & 0 & 0 & 0 \\
b_{1} & 0 & b_{2} & 0 & 0 & 0 & 1 & 0 & 0 \\
0 & 0 & 0 & 0 & 0 & 0 & 0 & Q & 0
\end{array}\right]
$$

Two lists of sums of products are generated by reading all paths from input to quantizer output with no feedback and from quantizer output to quantizer input with no input connected. These two lists are a symbolic equivalent to

$$
\begin{aligned}
& L_{0}=\frac{a_{1} a_{2} a_{3}}{(Z-1)^{3}}+\frac{a_{1} b_{2}}{(Z-1)}+\frac{b_{3} a_{3}}{(Z-1)}+b_{1} \\
& L_{1}=\frac{f_{1} a_{1} a_{2} a_{3}}{(Z-1)^{3}}+\frac{f_{1} a_{1} b_{2}}{(Z-1)}+\frac{f_{2} a_{2} a_{3}}{(Z-1)^{2}}+\frac{f_{3} a_{3}}{(Z-1)}
\end{aligned}
$$

which in turn can be used to equate two sets of equations for mapping to Zdomain polynomials representing $\mathrm{NTF}(\mathrm{Z})$ and $\mathrm{STF}(\mathrm{Z})$

$$
\begin{aligned}
& S_{2}^{L_{1}}=f_{1} a_{1} b_{2}+f_{3} a_{3} \\
& S_{1}^{L_{1}}=f_{2} a_{2} a_{3} \\
& S_{0}^{L_{1}}=f_{1} a_{1} a_{2} a_{3}
\end{aligned}
$$


and

$$
\begin{aligned}
& S_{3}^{L_{0}}=b_{1} \\
& S_{2}^{L_{0}}=a_{1} b_{2}+b_{3} a_{3} \\
& S_{1}^{L_{0}}=0 \\
& S_{0}^{L_{0}}=a_{1} a_{2} a_{3}
\end{aligned}
$$

The first set of equations maps the topology to $\operatorname{NTF}(Z)$ denominator (the pole set) so the system has to be precisely solved. The second set of equations maps the topology to the numerator of $\operatorname{STF}(Z)$ and therefore its fitting is not critical. An error here translates to shifting of STF $(\mathrm{Z})$ zeros, producing magnitude and phase bending. Some topologies cannot accommodate both equation sets, for example the topologies using only feedback or only feedforward coefficients do not offer any control over the $\operatorname{STF}(Z)$ zeros (see Chapter 2). If control of $\operatorname{STF}(\mathrm{Z})$ is critical, a high-complexity architecture complete with multiple feedbacks and feedforwards should always be used.

From $\operatorname{NTF}(Z)$ and $\operatorname{STF}(Z)$ expressions, the equation systems shown above are solved in iterations consisting of:

\section{INITIALIZE}

- set all integrator gains to arbitrary values (for example 1)

- determine how many equations are then over-constrained (only dependent on integrator gains)

- decide how many integrator gains are freedom degrees and set their values starting with the first one.

- calculate the $f_{1}$ coefficient (conversion gain) as the ratio $S_{0}^{L_{1}} / S_{0}^{L_{0}}$

\section{ITERATE}

- solve all equations which can be solved, starting with NTF equations and using the already-set integrator gains

- repeat until no other coefficient can be calculated in the current iteration

Remaining equations contain multiple independent variables and the equation systems are under-determined, so feedforward coefficients $b_{i}$ are treated like the integrator gains have been before, and the algorithm is applied again. After this step all the coefficients are determined.

Once the coefficients are fully determined, a time-domain simulation is performed with an input signal amplitude at the modulator's OVL. Starting with the first integrator, the peak output of each integrator is determined from simulation and the integrator coefficient $a_{i}$ is scaled with the ratio of the target peak output over the simulated peak output. After an integrator is scaled, another 
simulation is performed and the next integrator is scaled. Scaling the integrator coefficients requires a scaling of all the coefficients coupled to the integrator. For example, in the matrix in Eq. 5.37, if the coefficient of the first integrator $a_{1}$ has to be multiplied by $r$ then all the coefficients on columns 3 and 4 are divided by $r$ and all coefficients on rows 3 and 4 are multiplied by $r$. This leaves the mapping of the matrix to the target $\operatorname{NTF}(Z)$ and $\operatorname{STF}(Z)$ unchanged but scales the output of the first integrator to desired value, $r$ times larger than the previous result.

The cascaded solutions are treated as a set of single-loop solutions. Each loop in the cascaded is mapped and optimized individually. The coupling coefficients are calculated to bring the peak amplitude of the quantization noise of a loop at the OVL of the next one. The digital re-combination of the output digital streams is performed after scaling of each output stream in a digital filter using idealized $\operatorname{NTF}(\mathrm{Z})$ expressions [6].

\subsubsection{Performance Test}

Performance testing is based on statistics of SNDR and DR curves as functions of input signal level. Linear regressions are performed on each curve, from SNDR zero-crossing to the overloading level. The slope of SNDR is tested to be within $10 \%$ of the desired conversion gain (typically unity). A slope outside this range shows a strong dependency of quantization noise power of the input signal level, which is not desired. The intercept of the DR curve is then tested against the target $\mathrm{DR}$ value to insure the target $\mathrm{DR}$ is attained in the worst condition (DR extrapolated value at $0 \mathrm{~dB}$ input level). Finally, the peak regression residual of the DR curve is tested to be lower than $6 \mathrm{~dB}$ ( 1 bit) to insure the required integral non-linearity (INL). Finally, the overloading level is tested to be greater than $-15 \mathrm{~dB}$.

$S N D R\left(V_{i n}\right)$ and $D R\left(V_{i n}\right)$ curves for an architecture rejected by performance test algorithm are shown in Fig. 5.10. The dotted lines are the linear regression fitted values for both simulated curves. The drop in DR at high input levels (larger than -20dB) shows that the peak NTF value needed to reach the target DR is too high so premature clipping occurs [1]. The peak SNDR which still keeps a good overall INL is about $85 \mathrm{~dB}$ instead of almost $95 \mathrm{~dB}$, as shown by its absolute peak value. But the detection of this effect requires a set of linear regressions with each point in the curve used as higher limit, which would increase the computation time tenfold. Instead, based on the fact that the decrease in peak SNDR already disqualifies this solution, the simple yet effective criterion of peak regression residual limiting is used as rejection reason. The slope of DR fitted line shows that the test for DR intercept works toward rejecting the solution as well, even if peak regression residual would be passing its test. The test for SNDR slope also works toward rejecting the solution. 


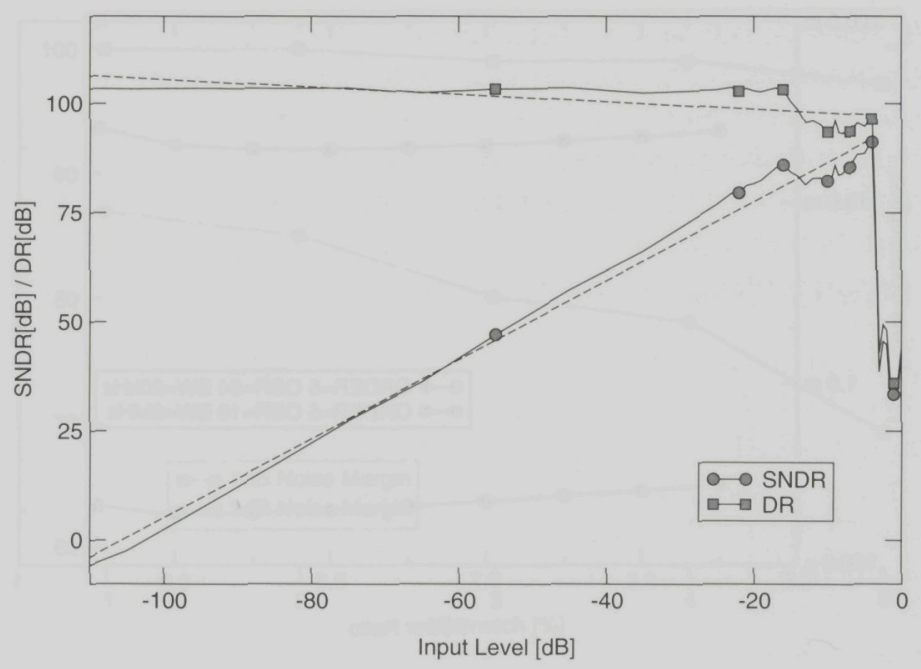

Figure 5.10: SNDR and DR curves for a rejected architecture (ORDER=3, $O S R=32, B I T S=2, L O O P S=3[1,1,1]), 2$ bits in the first loop

\subsubsection{Estimation of Power Consumption}

Each integrator in the loop has its power consumption characterized (for comparison purposes) by the $g_{m}$ of the operational amplifier insuring the required integration linearity. To calculate the total $g_{m}$ for a designed architecture-level solution, the passive components (resistors and capacitors) in each integrator have to be calculated from the noise budget and placed in the power models developed in Chapters 2 and 3 for SC and CT circuits respectively.

The noise budget of each integrator is allocated based on evaluation of total power consumption as a function of noise budget distribution across the converter. A part $R$ of the noise power of the previous integrator is allocated to the next in the loop

$$
\left(P_{n}\right)_{i}=R\left(P_{n}\right)_{i-1}
$$

and the value of $R$ is chosen to minimize total power consumption. Fig. 5.11 illustrates the dependence of power consumption of noise power allocation through the ratio $R$. The top curve is the power (expressed as the supply current) consumed for the total $g_{m}$ for a fifth-order, 3-loop, 2-2-1 architecture, with 5 bits in the first loop and 3 bits in the other two loops. It is operated at 16 times OSR for a signal bandwidth $B W$ of $2 \mathrm{MHz}$, as in [7]. It is worth noting the $25 \%$ reduction in current consumption by optimization of $R$ and the fact that the minimum current, of $14 \mathrm{~mA}$, is well related to the reported consumption of $36 \mathrm{~mA}$ if folded cascode amplifiers are used [8]. The second curve in Fig. 5.11 
Computer Aided Design of Sigma-Delta ADCs

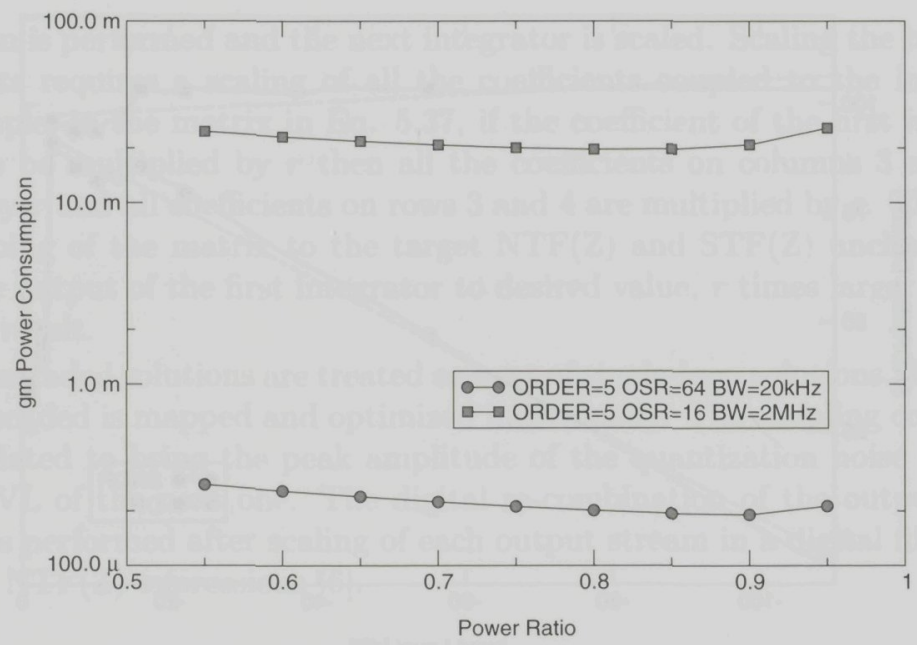

Figure 5.11: Power consumption for $g_{m}$ as function of noise power ratio $R$

is the current consumption for a fourth-order, one-bit single-loop $\Sigma \Delta$ ADC for audio applications $(B W=20 \mathrm{kHz})$. The supply current estimated in the best case also matches the design reported in [8].

\subsubsection{Yield Analysis and Optimization}

From the accepted solutions, a top-ten set is chosen based on the ratio of peak SNDR and the power consumption in opamps measured by the total $g_{m}$. The solutions set is reduced because the Monte-Carlo yield analysis and optimization is likely to take a long processing time. For each top-ten solution, the MonteCarlo analysis varies the $\Sigma \Delta \mathrm{ADC}$ coefficients using a normal distribution with the standard error supplied by the user. The user can also specify a spread $3 \sigma$ value along with the coefficient-to-coefficient mismatch $3 \sigma$ value. Required minimal capacitor and maximal resistor values are specified for the latter $3 \sigma$. The spread is used in the case of CT designs to simulate the effect of RC product spread over design performance.

A few hundreds of Monte-Carlo simulation steps are run for the top-ten solutions and the performance tests explained in sub-Section 5.2.3 are applied. The single-loop solutions can be designed from the early stages to give $100 \%$ yield with relaxed matching requirements by controlling the difference between the quantization noise power and in-band white noise power. Fig. 5.12 shows the yield variation for a fourth-order, single-bit design when this difference is $1 \mathrm{~dB}$ and $3 \mathrm{~dB}$. It can be seen that, in this case, if the white noise is $3 \mathrm{~dB}$ above the quantization noise power inside the signal bandwidth no significant yield 


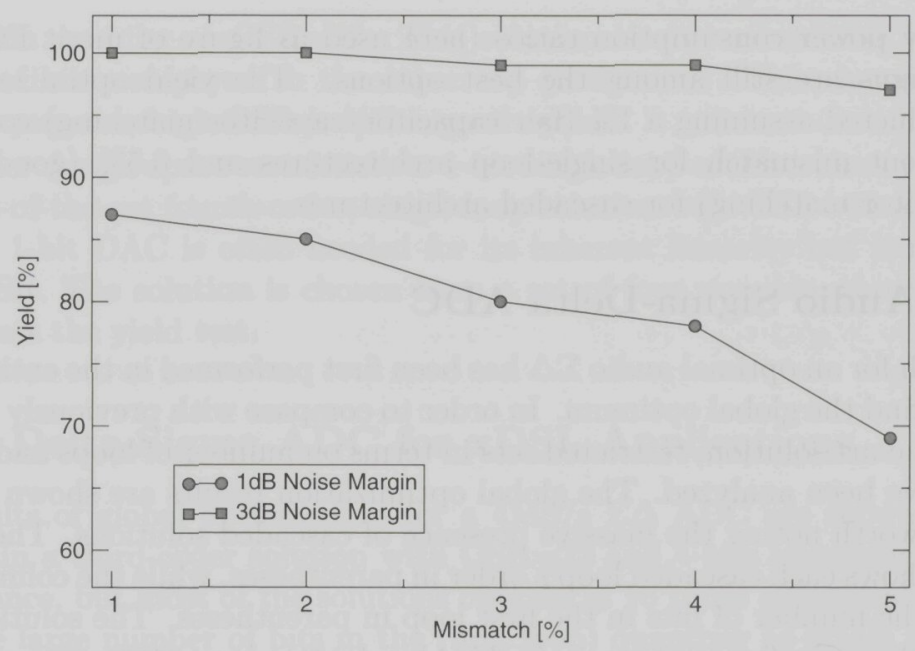

Figure 5.12: Yield of a single-loop solution (ORDER=4, BITS=1) as a function of coefficient mismatch at different white-quantization noise margins

variation is observed up to $4 \%$ coefficient mismatch.

For cascaded solutions however, the yield can be corrected by increasing the number of bits in the first loop. This decreases the quantization noise power in the first loop and therefore the power of the mismatch-induced, low-order shaped noise leaking to the output. When the yield is lower than $90 \%$ the number of bits in the first loop in the cascade is increased and another Monte-Carlo yield analysis is started.

\subsection{Design Examples}

Two examples are presented to show the effectiveness of global optimization through exhaustive design-space exploration. The first one is an audio $\Sigma \Delta$ ADC powered at $1.5 \mathrm{~V}$ with rail-to-rail input and $1.5 \mathrm{~V}$ reference voltage. A designed circuit has been reported [8] which consumes $0.95 \mathrm{~mW}$ (only the analog part, without voltage reference buffers) for a DR of $98 \mathrm{~dB}$ and peak SNDR of $89 \mathrm{~dB}$ at a signal bandwidth of $20 \mathrm{kHz}$. The second one is an ADC for xDSL applications, powered at $2.5 \mathrm{~V}$, also with rail-to-rail input and reference voltage equal to the supply voltage. The signal bandwidth is $2 \mathrm{MHz}$. A design has been reported which consumes $90 \mathrm{~mW}$ in the analog circuits to attain $95 \mathrm{~dB}$ DR and $90 \mathrm{~dB}$ peak SNDR.

The designs mentioned above are state-of-the art examples. The results presented here show that other architectures can offer better peak SNDR ver- 
sus supply power consumption ratios (here used as figure of merit FOM) but these designs are still among the best options. The yield optimization has been conducted assuming a 1\% (fair capacitor/capacitor matching) coefficientto-coefficient mismatch for single-loop architectures and $0.5 \%$ (good capacitor/capacitor matching) for cascaded architectures.

\subsubsection{Audio Sigma-Delta ADC}

The search for an optimal audio $\Sigma \Delta$ has been first performed in the entire design space, to find the global optimum. In order to compare with previously reported state-of-the-art solution, restricted sets in terms on number of loops and number of bits have been analyzed. The global optimization results are shown in Table 5.1. It is worth noting the massive presence of cascaded solutions. The column LOOPS shows each cascaded loop's order in parentheses, while the column BITS contains the number of bits in the first loop in parentheses. The solutions have virtually the same figure-of-merit FOM. All solutions have OSR=32 and a large number of bits in the first loop, which increases the FOM value by increasing the overloading level with no power costs.

Table 5.1: Global solutions for Audio $\Sigma \Delta A D C$

\begin{tabular}{|c|c|c|c|c|c|}
\hline ORDER & LOOPS & BITS & OSR & SNDR & FOM \\
\hline 4 & $2(2-2)$ & $2(6)$ & 32 & 97.5 & 119.8 \\
\hline 4 & $3(2-1-1)$ & $2(5)$ & 32 & 97.3 & 119.5 \\
\hline 5 & $3(2-1-2)$ & $1(7)$ & 32 & 98.5 & 120.2 \\
\hline 5 & $2(2-3)$ & $4(7)$ & 32 & 98.5 & 119.8 \\
\hline 5 & $2(2-3)$ & $5(7)$ & 32 & 98.1 & 119.5 \\
\hline
\end{tabular}

Table 5.2 contains the optimization results for a set of solutions restricted by the number of loops (LOOPS $=1$ ). The best solution is the third order, 4-bit

Table 5.2: Audio ADCs with LOOPS=1

\begin{tabular}{|c|c|c|c|c|}
\hline ORDER & BITS & OSR & SNDR & FOM \\
\hline 3 & 6 & 16 & 95.3 & 117.6 \\
\hline 3 & 8 & 16 & 96.3 & 117.8 \\
\hline 3 & 4 & 32 & 95.5 & 118.4 \\
\hline 4 & 8 & 8 & 95.6 & 116.4 \\
\hline 4 & 5 & 16 & 96.0 & 117.8 \\
\hline 5 & 4 & 16 & 95.4 & 116.5 \\
\hline 5 & 6 & 16 & 96.2 & 115.9 \\
\hline
\end{tabular}


loop, again working at OSR $=32$. This solution is also remarkable by its low number of bits compared to the other top performers. This shows that, for audio frequencies, high-OSR is still a good option.

Further design space restriction to 1-bit single-loop architectures yields only the state-of-the-art fourth-order, single-loop solution with OSR $=64$ reported in [8]. The 1-bit DAC is often needed for its inherent linearity but it requires a larger OSR. The solution is chosen from a set of four possible, three of which do not pass the yield test.

\subsubsection{Delta-Sigma ADC for xDSL Applications}

The results of global optimization for a $4 \mathrm{MS} / \mathrm{s} \Sigma \Delta \mathrm{ADC}$ are shown in Table 5.3. Again a third-order solution with $\mathrm{OSR}=32$ and 4-bits quantizer has good performance, but most of the solutions operate at 16 times oversampling. They also have large number of bits in the (first loop) quantizer to attain high overloading levels. The FOM values are $20 \mathrm{~dB}$ smaller than the ones obtained with the audio ADCs because of increased sampling frequency.

Table 5.3: Global solutions for xDSL $\Sigma \Delta A D C$

\begin{tabular}{|c|c|c|c|c|c|}
\hline ORDER & LOOPS & BITS & OSR & SNDR & FOM \\
\hline 3 & 1 & 4 & 32 & 96.5 & 102.7 \\
\hline 4 & $2(2-2)$ & $4(8)$ & 16 & 97.4 & 103.1 \\
\hline 4 & $3(2-1-1)$ & $4(7)$ & 16 & 98.1 & 102.7 \\
\hline 5 & $2(2-3)$ & $3(8)$ & 16 & 96.6 & 102.1 \\
\hline 5 & $2(2-3)$ & $4(6)$ & 32 & 97.1 & 101.7 \\
\hline 6 & $3(2-2-2)$ & $2(8)$ & 16 & 97.4 & 102.6 \\
\hline
\end{tabular}

Table 5.4: $x D S L A D C$ s with $\mathrm{ORDER}=5, \mathrm{OSR}=16$

\begin{tabular}{|c|c|c|c|}
\hline LOOPS & BITS & SNDR & FOM \\
\hline $2(4-1)$ & $2(5)$ & 93.1 & 98.0 \\
\hline $3(2-2-1)$ & $2(6)$ & 97.1 & 102.6 \\
\hline $3(2-2-1)$ & $3(5)$ & 95.7 & 101.0 \\
\hline $3(2-2-1)$ & $4(6)$ & 97.8 & 102.5 \\
\hline 1 & 5 & 96.6 & 100.4 \\
\hline $3(2-2-1)$ & $5(5)$ & 98.1 & 102.6 \\
\hline 1 & 6 & 96.5 & 99.6 \\
\hline $3(2-2-1)$ & $6(6)$ & 98.2 & 101.7 \\
\hline
\end{tabular}


To avoid solutions like the ones requiring 32 times OSR, the designer can conduct the search in a limited space, for example for (ORDER=5, OSR=16). Furthermore, the number of bits can be limited, for example to 6 , to keep a low DAC complexity. The solutions for this search are shown in Table 5.4. They are all cascaded $\Sigma \Delta$ ADCs except two which, even with their low-FOM, can be good choices for low-voltage, mismatch-tolerant designs.

The best are the three-loops with a 2-2-1 configuration. The state-of-theart solution reported so far [7] is among them. During initial optimization stages, only 3 bits were needed in the first loop, but yield optimization reached the 5-bit solution reported in [7], the increase being needed to accommodate capacitor/capacitor mismatch effects. 


\section{References}

[1] R. Schreier Simulation and Design Examples, Delta Sigma Converters for Telecom, Mead course, Switzerland, July 2000.

[2] S.R. Norsworthy, R. Schreier, G.C. Temes, Delta-Sigma Data Converters, IEEE Press, 1997.

[3] W.H. Press et.al., Numerical Recipes in C; The Art of Scientific Computing, Cambridge University Press, Cambridge, U.K., 1992.

[4] L.A. Zadeh, Linear System Theory; The State Space Approach, McGraw Hill, New York, U.S.A., 1963.

[5] G. Lago, L.M. Benningfield, Circuit and System Theory, John Wiley \& Sons, U.S.A., 1979.

[6] F. Medeiro, B.P. Verdu, A.R. Vasquez, Top-Down Design of HighPerformance Sigma-Delta Modulators, Kluwer Academic Publishers, 1999.

[7] K. Vleugels, S. Rabii, B.A. Wooley, A $2.5 \mathrm{~V}$ Broadband Multi-Bit $\Sigma \Delta$ Modulator with 95dB Dynamic Range, ISSCC 2001 Digest of Technical Papers, Feb. 2001.

[8] A.L. Coban, P.E. Allen, A $1.5 \mathrm{~V} 1.0 \mathrm{~mW}$ Audio $\Sigma \Delta$ Modulator with 98dB Dynamic Range, Digest of Technical Papers of Intl. Solid-State Circuits Conference, pp. 50, Feb. 1999. 
REFERENCES

128 Systematic Design of $\Sigma \Delta$ ADCs 


\section{Sigma-Delta ADC for Audio Applications}

Most of the present cordless and mobile telephony sets and audio multimedia devices are employing an electret microphone as audio input device. These microphones are small, reliable, cheap and have electro-acoustical characteristics well suited for low- to moderate-quality audio applications. The microphones can be manufactured in different sizes, larger diameter devices showing better acoustic sensitivity at the expense of increasing costs, especially when used in mobile phones [1].

A schematic of the A/D conversion chain commonly used in mobile phones is shown in Fig. 6.1, (left). The JFET packaged along with the microphone $M$ is used as voltage-to-current converter. The signal is AC coupled to the Codec chip by a large-valued capacitor $C$. The cost of the system can be reduced by integrating $R$ along with the JFET and designing new Codec chips that can be connected directly to the JFET drain [3].

However, other disadvantages of this system remain. At approximately $60 \mathrm{~dB}$ dynamic range and $1 \%$ total harmonic distortion the performance is limited by the JFET's noise and nonlinearity, even when it is biased at a relatively high drain current. The transconductance $g_{m}$ of the JFET is not very large and the voltage gain is $g_{m} R, R$ being limited in value by the available voltage span to $V d d$. With decreasing supply voltages, the voltage gain available decreases as well, hence the amplitude of the signal at JFET's drain. This analog signal is sensitive to externally generated electrical noise because it is connected through an un-shielded, single wire to the Codec chip. This sensitivity is especially not desired when the microphone is used in mobile phone designs where digital and $\mathrm{RF}$ components generate powerful noise fields.

The concept developed in this project is illustrated in Fig. 6.1, (right). 

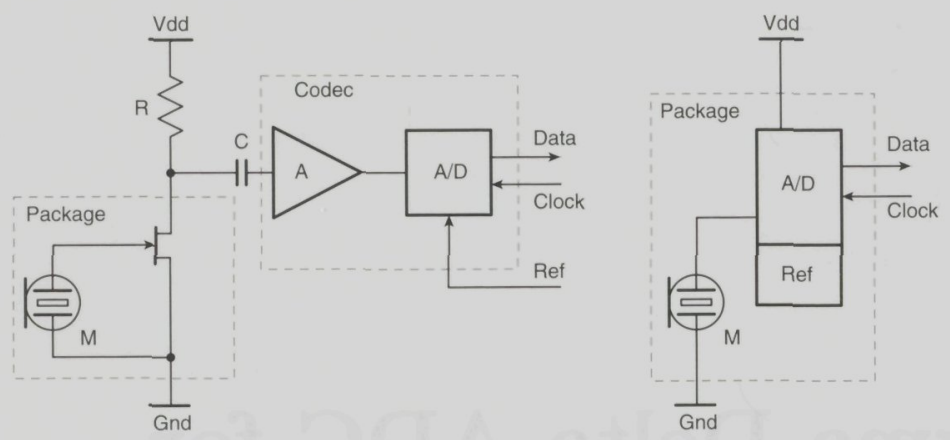

Figure 6.1: Conventional (left) and new (right) approach for $A / D$ conversion of electret microphone signal

The JFET is replaced by a chip containing an ADC and a voltage reference. The ADC is connected directly to the microphone, without making use of an amplifier or filter for signal conditioning. By moving the ADC closer to the microphone the most sensitive node in the system, namely the output of the microphone, remains inside the shielding metal capsule and therefore protected from external noise sources. There is no need of external (discrete) components anymore.

The voltage reference is placed on the same chip with the ADC, so no sensitive analog connections are taken outside the metal package. The number of external connections is also minimized: only the digital Clock input and 1-bit Data output are needed besides ground and supply.

Section 6.1 describes the electret microphone as a high-impedance voltage source. Section 6.2 covers the various aspects of system design, explaining how the constraints are leading to a certain architectural choice for the $\Sigma \Delta$ ADC. Section 6.3 gives a detailed description of the circuits used in the ADC. The analysis of conversion linearity, limited by the performance of the first integrator, is presented in Section 6.4. The measurement results are presented in Section 6.5 .

\subsection{The Electret Microphone}

The structure of the electret microphone is shown in Fig. 6.2. The capacitor inside is built as one stiff, teflon-covered plate and one flexible membrane consisting of mylar covered with a conductive material. The sound waves entering the Sound Port make the flexible membrane vibrate, thus inducing capacitance variations. The electret is the teflon layer. 


\subsection{The Electret Microphone}
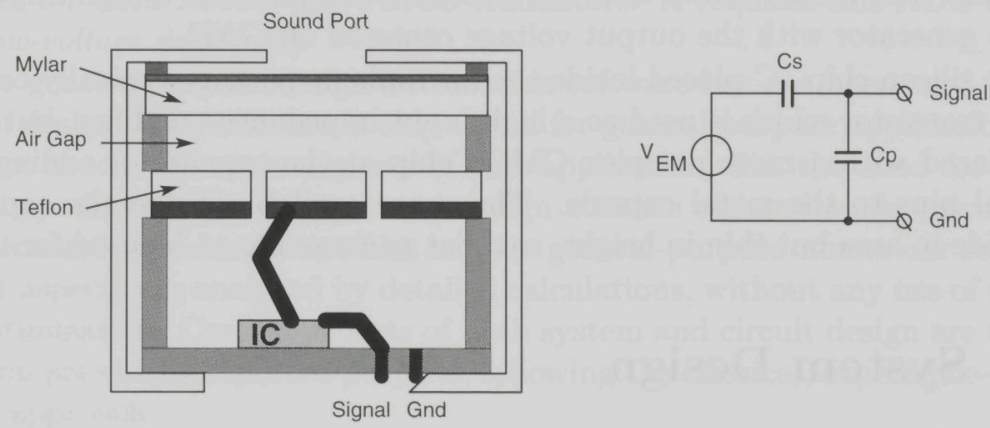

Figure 6.2: Electret Microphone Structure

Its fixed charge combined with the pressure-sensitive capacitor generate a voltage between the two plates which is given by

$$
V_{E M}=E_{0} \bar{h}+\frac{\sigma_{L} d}{\epsilon_{d}}
$$

The microphone voltage $V_{E M}$ is thus proportional to the built-in electrostatic field $E_{0}$ and the equivalent distance between the plates $\bar{h}$ added to a constant term given by factory-induced electret charge $\sigma_{L}$ and the thickness and electrical permittivity of the teflon $\epsilon_{d}$. The equivalent distance between the plates is defined as

$$
\bar{h}=h+e \frac{\epsilon_{0}}{\epsilon_{e}}+d \frac{\epsilon_{0}}{\epsilon_{d}}
$$

calculated for a stack of three layers with different permittivities. Parameter $h$ is the space between the plates, $e$ is the thickness of mylar and $d$ is the thickness of teflon, with each material's permittivity denoted by $\epsilon_{0}$ for air, $\epsilon_{e}$ for mylar and $\epsilon_{d}$ for teflon.

Without acoustic signal, the voltage between the microphone plates is zero, which can be used to define the electret equivalent voltage

$$
V_{E}=-\left.E_{0} \bar{h}\right|_{V_{E M}=0}=\frac{\sigma_{L} d}{\epsilon_{d}}
$$

Voltage $V_{E}$ is in the range of $200 \mathrm{~V}$ to $300 \mathrm{~V}$, its large value improving microphone sensitivity.

The electrical model of the microphone includes a voltage generator, a series and a parallel capacitor as shown in Fig. 6.2. The voltage generated is in the order of hundreds of milivolts. The two capacitors have values of approximately 
$5 \mathrm{pF}$ each. The model shows that the electret microphone is a high-impedance voltage generator with the output voltage centered on GND.

The silicon chip $I C$ placed inside the microphone package typically contains a FET transistor which is used as a high input-impedance amplifier, but it can be replaced with a more complex CMOS chip at the expense of adding more external pins to the metal capsule. The space available inside the capsule is very wide in area but thin in height, so a flat package has to be used for the IC.

\subsection{System Design}

\subsubsection{Project Specifications}

The small number of available pins and the need for a high conversion resolution at audio frequencies makes the choice of a 1-bit $\Sigma \Delta$ ADC the most suitable one for this application. The design challenges at system level consist of connecting the $\Sigma \Delta$ ADC directly to the microphone and to the on-chip voltage reference without making use of fast-slewing amplifiers or buffers. The $\Sigma \Delta$ ADC connected to the microphone requires a high impedance input to isolate the electret material from the rest of the circuit. This single-ended input connects capacitively to the $V_{E M}$ signal source (Fig. 6.2). The voltage generated by the microphone has a peak amplitude of $125 \mathrm{mV}$ on a load capacitor of $2 \mathrm{pF}$. The signal is centered on the ground potential, therefore the input of the $\Sigma \Delta$ $\mathrm{ADC}$ requires a voltage input range which extends below ground with the device powered from a single positive voltage source.

The output digital code of an ADC represents the ratio of the input signal and the reference voltage. Therefore, with a small input amplitude, a conversion gain has to be designed in the ADC so full-scale codes are reached when a large reference voltage is applied. While the microphone generates peak amplitudes of $125 \mathrm{mV}$, a conversion gain of $14 \mathrm{~dB}$ should be implemented by the $\Sigma \Delta$ ADC for a $1.25 \mathrm{~V}$ reference voltage and an expected overloading level of $-6 \mathrm{dBR}$. A low ripple of the frequency response inside the signal bandwidth is preferred, so the digital filter following the converter does not have to implement amplitude corrections.

The target dynamic range of $84 \mathrm{~dB}$ exceeds the dynamic range achievable by the JFET-based solution. The converted signal bandwidth has to be $11 \mathrm{kHz}$, so that the microphone can be used for both telephony and multimedia applications. White noise should dominate the quantization noise from $\mathrm{DC}$ to $11 \mathrm{kHz}$ for an increase of $5 \mathrm{~dB}$ in DR when the microphone is used for only the telephony bandwidth of $3.5 \mathrm{kHz}$. For a $\Sigma \Delta \mathrm{ADC}$ design this requires the quantization noise to fall below the device-generated (mainly thermal) noise inside the signal band.

The supply voltage ranges between $1.8 \mathrm{~V}$ and $2.5 \mathrm{~V}$. For the $0.5 \mu \mathrm{m}$ CMOS technology used, the lower supply voltage limit equates less than two threshold 
voltages for both NMOS and PMOS transistors. It requires this $\mathrm{ADC}$ to be a true low-voltage design [2].

The general-purpose design automation software has not been used for this custom design. The software is only useful for general-purpose applications and does not model the main aspects of this application, like the need for a high input impedance. The system-level design consists of hand-made calculations and custom-made simulation files ran by general-purpose numerical software. Circuit aspects are analyzed by detailed calculations, without any use of numerical optimization. Certain aspects of both system and circuit design are treated based on previously reported projects, following the classical, experience-driven, design approach.

\subsubsection{Switched-Capacitor vs. Continuous-Time Loop Fil- ter}

As in all $\Sigma \Delta$ designs, a choice has to be made between a switched-capacitor (SC) loop filter and a continuous-time (CT) one.

At architecture-level, the SC circuits offer better stability (higher overloading levels for the same NTF aggressiveness) and easier timing of quantizer and DAC signals inside the loop. They are also less sensitive to circuit non-idealities than the CT counterparts.

At circuit-level, the CT designs are more appealing. While all SC circuits need a driver in front of the integrator to load the sampling capacitors which perform the voltage-to-charge conversion [4], CT integrators can be designed with a high input impedance [3]. Their additional advantage is a lower noise bandwidth than a SC counterpart, so a better performance versus power ratio can be achieved. Furthermore, the SC circuits require high-bandwidth amplifiers to be used in order to transfer the charge from sampling to integrating capacitors at required settling accuracy during one clock cycle. CT circuits require less unity-gain bandwidth in the amplifiers for the same operating speed. On the $\Sigma \Delta$ feedback side, the SC DACs are also made of switched capacitors so the reference voltage has to be buffered to attain the required sampling speed. The DACs for CT integrators can be implemented with current sources which do not load the voltage reference dynamically.

Therefore, a mixed CT-SC loop filter has been found to be the most convenient choice for this project. A system-level schematic of the designed chip is shown in Fig. 6.3. A fourth-order, single-loop $\Sigma \Delta$ modulator performs the function of A/D conversion. Only one bit of data is delivered at the output on each clock cycle, therefore a single-bit quantizer (comparator) is used. A large oversampling ratio (OSR) in conjunction with higher-order noise shaping make it possible to attain a high conversion resolution.

The $\Sigma \Delta$ modulator is connected to one plate of the microphone $M$ with the single-ended input of the first integrator. As one plate of the microphone is 


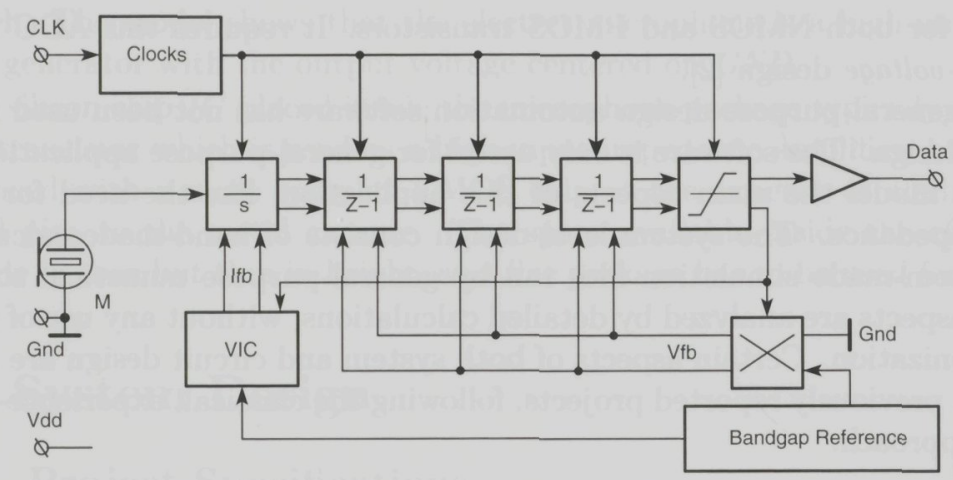

Figure 6.3: System-level schematic of the digital microphone chip

structurally connected to ground, there is no possibility to differentially connect the microphone to the modulator. However, for good performance at low supply voltages, the rest of the modulator is designed with differential circuitry. Singleended-to-differential conversion takes place at the level of the first integrator, as will be explained in sub-section 6.3.3.

The first integrator is a continuous-time circuit with a high input impedance. The higher-order integrators, free from special constraints, are designed as SC integrators which perform well when biased at low supply voltage. The 1-bit quantizer inside the $\Sigma \Delta$ loop is a differential comparator.

The on-chip bandgap reference supplies a voltage which is converted to a differential current by the voltage-to-current converter $(V I C)$. This approach does not load the bandgap reference dynamically, therefore allowing low power consumption in the voltage reference buffer. The buffer has to drive only the scaled-down capacitors of the higher-order integrators.

All the clock phases required by the SC circuits are derived on-chip from a master clock line supplied from outside.

\subsection{Sigma-Delta ADC Design}

\subsubsection{Topology}

The full architecture of the $\Sigma \Delta$ modulator is shown in Fig. 6.4. This architecture allows for good control of the noise transfer function (NTF) as well as for a good control of signal transfer function (STF) by using both the feedback coefficients $f_{1} \cdots f_{4}$ and the feed-forward coefficients $b_{1} \cdots b_{3}$. No feed-forward coefficient is connected at the input of the converter, leaving only the first integrator to load the electret microphone. 


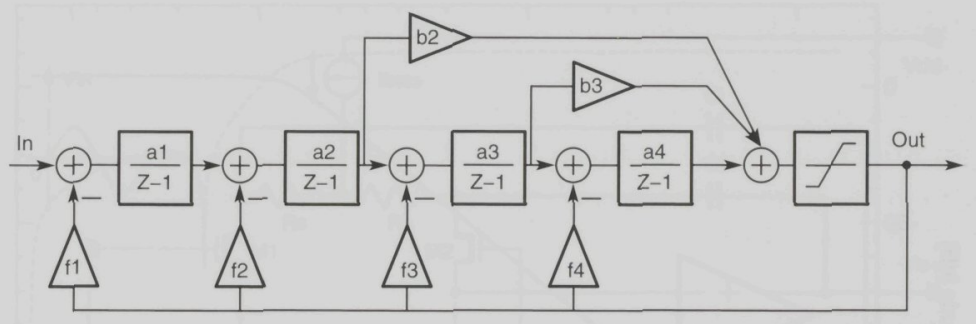

Figure 6.4: Modulator topology

The first three integrator coefficients $a_{1} \cdots a_{3}$ can be chosen arbitrarily and all the other coefficients are then calculated to map the $Z$-domain transfer functions $\operatorname{NTF}(Z)$ and $\operatorname{STF}(Z)$ to the physical structure. The coefficients are designed to bound the integrator outputs within $10 \%$ to $80 \%$ of the voltage between ground and the supply voltage when the modulator is not overloaded. Integrator clipping to supply rails is used to limit oscillations when the input is larger than the overloading level. A value of $f_{1}$ lower than unity sets the desired in-band conversion gain.

The modulator is designed to oversample the $11 \mathrm{kHz}$ signal bandwidth at 64 times, with a clock frequency of $1.408 \mathrm{MHz}$. Under these conditions, the fourth-order single-bit $\Sigma \Delta$ attains a total in-band quantization noise power of $-92 \mathrm{~dB}$. As a result, the white noise having a projected in-band power of $-84 \mathrm{~dB}$ will dominate the quantization noise and function as dither to prevent the idle tones at $-115 \mathrm{~dB}$ to become audible. The reference voltage considered for all behavioral modeling is $1.5 \mathrm{~V}$. The supply voltage is $1.8 \mathrm{~V}$ and all circuits and signals are considered fully differential.

\subsubsection{NTF and STF Design}

The NTF is designed following Lee's rule [5], with a gain slightly larger than the $3 \mathrm{~dB}$ recommended for stability reasons. The more aggressive NTF has been designed to push the in-band quantization noise as low as possible compared to the projected thermal noise. The value of NTF gain near $f_{s} / 2$ has been chosen based on system-level simulations for millions of clock cycles, designed to observe low-frequency oscillations proving loop instability. No in-band NTF zeros are used, as they are susceptible of generating extremely low-valued loop coefficients in connection with the large oversampling ratio used [6].

The advantage of a high-complexity architecture is illustrated in Fig. 6.5. As opposed to simpler architectures [6], complete control of in-band STF magnitude ripple is achieved. The designed flatness of the STF magnitude is better than $0.1 \mathrm{~dB}$ from DC to $11 \mathrm{kHz}$. The pair of zeros in the STF characteristic also 


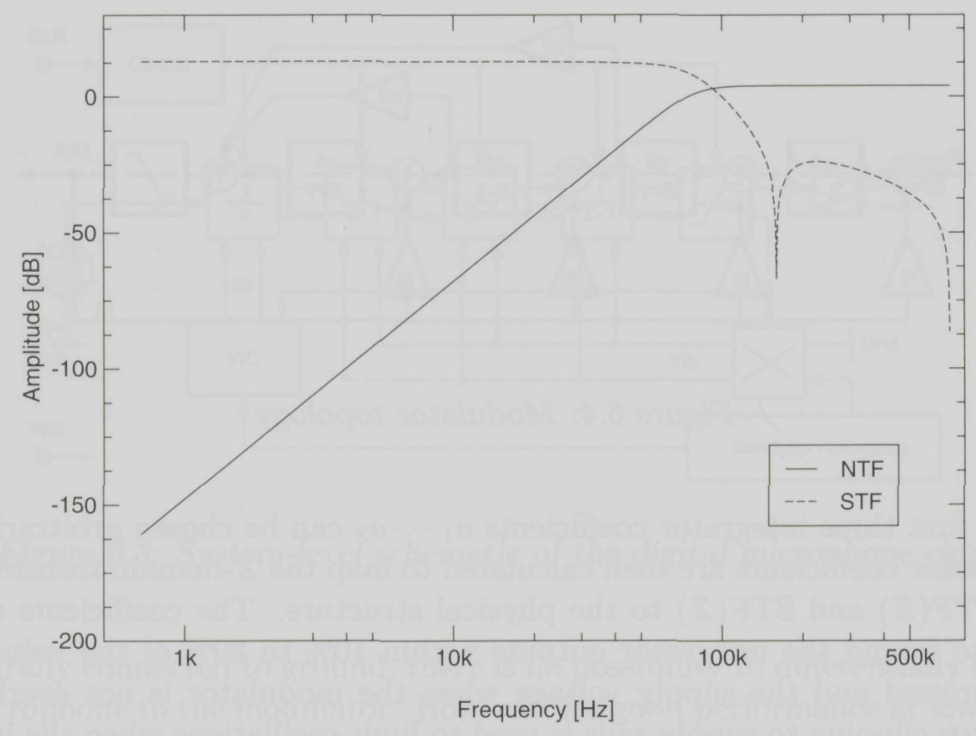

Figure 6.5: Magnitude characteristics of STF and NTF

provide for high-frequency roll-off, which increases rejection of out-of-band input signals. This compensates for the complete absence of input-signal conditioning. The in-band STF gain is $12 \mathrm{~dB}$, which translates the peak input signal of $125 \mathrm{mV}$ $(-21 \mathrm{dBR})$ to the output overloading level of the modulator of $-9 \mathrm{dBR}$.

\subsubsection{High Input Impedance Continuous-Time Integrator Signal Path}

Conventional SC integrators [4] and CT integrators [7] do not need a high input impedance because in most applications a signal buffer or amplifier is already available in front of the $\Sigma \Delta$ modulator. To connect the modulator directly to the microphone, the custom integrator shown in Fig. 6.6 and Fig. 6.8 has been designed. The integrator has an instrumentation amplifier input stage which works as a voltage-to-current converter and has high input impedance.

The single-ended microphone signal Vin (Fig. 6.6) is connected to the gate of the PMOS transistor M1. The use of PMOS devices provides an input range of more than $200 \mathrm{mV}$ below ground, enough to accommodate the microphone signal. The transistor pair $M 1, M 2$ repeats the voltage Vin onto the resistors $R s$. The resulting current is integrated by the operational amplifier on the feedback capacitors $\mathrm{C} i$. The two-stage class-A output amplifier keeps its differential input voltage close to zero by balancing the drain currents of $M 1$ and M2. In the 


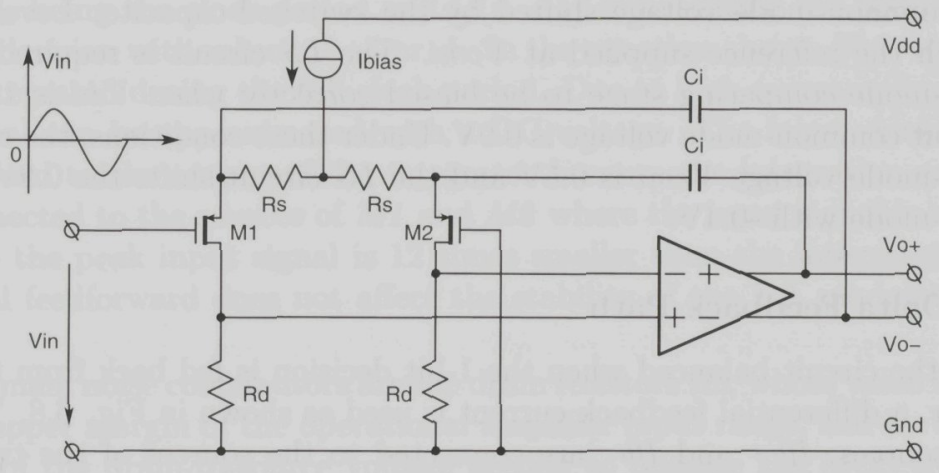

Figure 6.6: Microphone signal integration by single-ended input CT integrator

single-ended-to-differential conversion which takes place, balancing of the circuit greatly reduces common-mode cross talk and even harmonic distortion.

The amplifier used in the first integrator is designed for maximal output swing and high in-band gain. Its schematic is shown in Fig. 6.7. It consists of a PMOS input pair M1-M2 which is designed to operate in weak inversion for maximal transconductance at a given tail current. The input pair is cascoded to increase the impedance level at the output of the first stage so the offset and noise of the output stage has reduced effect. The DC gain in-band is also boosted without increasing power consumption. The second stage has M3 and M4 as active transistors to drive the load capacitors. Output common-mode voltage is controlled by a common-mode feedback loop which compares the

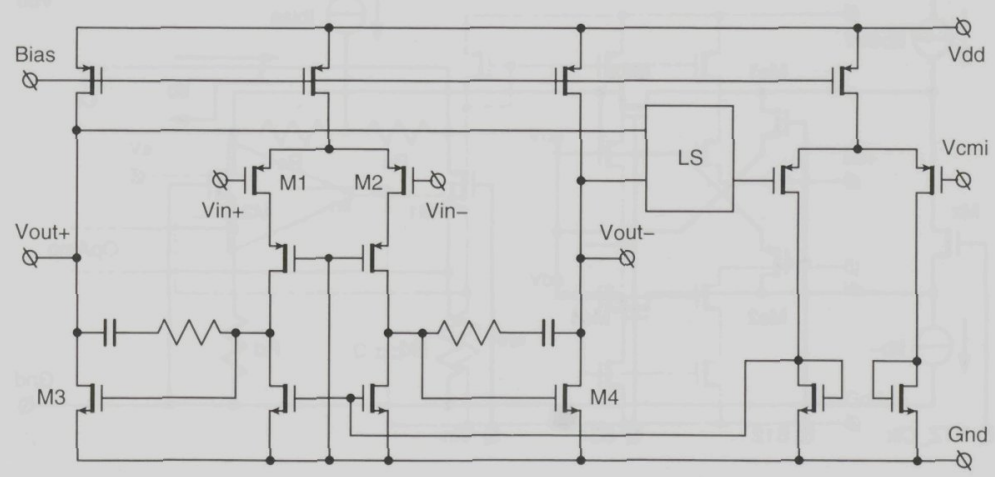

Figure 6.7: Two-stage class-A amplifier used in the first integrator 


\section{Sigma-Delta ADC for Audio Applications}

output common-mode voltage shifted by the switched-capacitor Level Shifter $(L S)$ with the reference supplied at $V c m i$. The $L S$ circuit is required for the common-mode comparing stage to be biased correctly when $V d d$ is $1.8 \mathrm{~V}$ and the output common-mode voltage is $0.9 \mathrm{~V}$. Under these conditions, the reference common-mode voltage $V c m i$ is $0.5 \mathrm{~V}$ and the $L S$ circuit shifts the $0.9 \mathrm{~V}$ output common-mode with $-0.4 \mathrm{~V}$.

\section{Sigma-Delta Feedback Path}

To keep the circuit balanced when the 1-bit decision is fed back from the loop quantizer, a differential feedback current is used as shown in Fig. 6.8. The two current sources, $I f b+$ and $I f b$-, are connected to the sources of the two MOS transistors according to the quantizer decision. The switches Ms1 $\cdots M s 4$ are activated by the digital signals $S 12$ and $S 34$, directing the differential feedback current $I f b$ to flow from the source of $M 1$ to the source of $M 2$ or vice versa. The feedback current is integrated by the operational amplifier on the two capacitors $\mathrm{Ci}$ in the same manner as the signal current. The functioning of the return-tozero clock $R T Z_{-}$clk will be explained in a following section. All three clocking signals are boosted by local charge-pump voltage doublers to $3.4 \mathrm{~V}$.

The feedback current sources are cascoded to increase their output impedance, so the supplied current is not dependent on the microphone voltage signal when an Ifb source is connected to the source of $M 1$ (Fig. 6.8).

With the signal Vin at the input and the $\Sigma \Delta$ feedback applied, the output of the integrator at an arbitrary clock index $n$ is:

$$
V_{\text {out }}(n)=V_{\text {in }}+V_{\text {out }}(n-1)+\frac{V_{\text {in }}}{R s} \frac{T_{c l k}}{C i}-Q(n-1) \frac{I_{f b} T_{c l k}}{C i}
$$

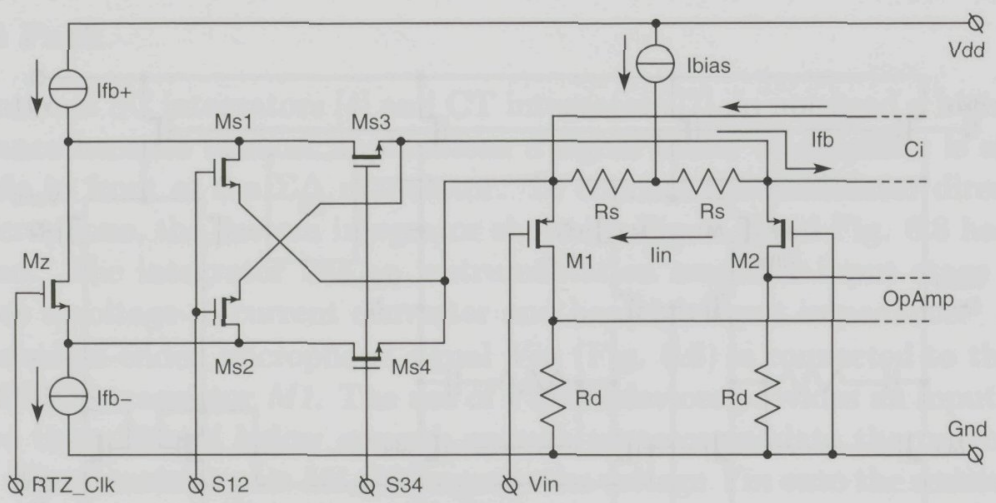

Figure 6.8: One-bit $\Sigma \Delta$ feedback integration by single-ended input CT integrator 
with $T_{c l k}$ being the clock period and $Q(n-1)$ representing the previous comparator decision with values of +1 or -1 . In the equation above, Ifb is considered to be integrated during the full clock period. Due to high oversampling, the input signal can be approximated with a DC voltage. The input signal itself is fed forward to the output of the integrator because the integration capacitors are connected to the sources of $M 1$ and $M 2$ where the input signal is repeated. Because the peak input signal is 12 times smaller than the integrator output, the local feedforward does not affect the stability of the $\Sigma \Delta$ modulator or its DR.

The main noise contributors are the drain resistors $R d$, whose value is limited by the upper margin of the operational amplifier input range, and by the lower margin of the drain-to-source voltage needed to operate the MOS transistors off the linear region. The other noise sources are the $R s$ resistors, whose values can only be decreased at the expense of increasing the size of the $\mathrm{C} i$ capacitors, and the MOS transistors M1 and M2, which are operated at drain currents of $7.5 \mu \mathrm{A}$ to allow the use of larger-value $R d$ resistors. Approximately $90 \%$ of the noise power is generated by the mentioned devices.

To generate the biasing for the feedback current sources from the bandgap voltage, the voltage-to-current $(\mathrm{V} / \mathrm{I})$ converter shown in Fig. 6.9 is used. The operational amplifier repeats the bandgap voltage $V a$ on the resistor Rref, sustaining the resulting current through the NMOS transistor connected in the loop. The noise bandwidth of the $\mathrm{V} / \mathrm{I}$ converter is reduced by the capacitive load of the one-stage amplifier. Variation of Rref over temperature does not degrade the $\Sigma \Delta$ performance as long as Rref is matched with the resistors $R s$ (Fig. 6.6). The matching ensures a constant signal/feedback ratio which guarantees the mapping of NTF poles to loop filter to be independent of temperature variations or process spreads.

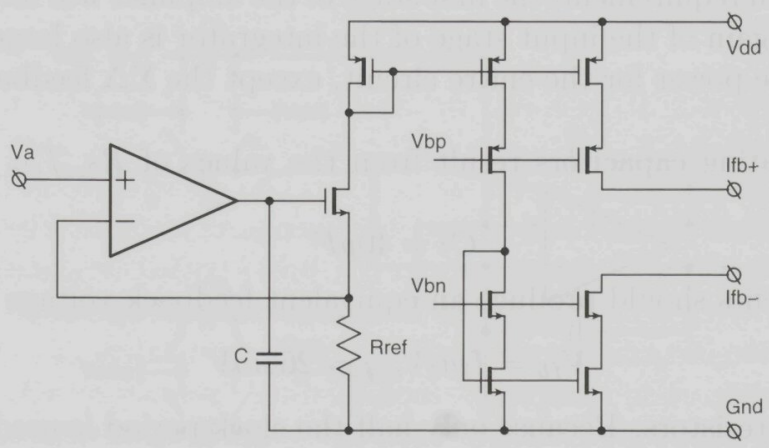

Figure 6.9: $\mathrm{V} / \mathrm{I}$ converter used to generate the feedback current for the first integrator 


\section{Sizing of the First Integrator}

The noise power added in the circuit by the first stage of the integrator can be calculated as

$$
\overline{v_{n}^{2}}=16 K T f_{b}\left(R_{S}+\frac{2}{3 g_{m}}\right)+8 K T f_{b} \frac{1}{R_{D}}\left(R_{S}+\frac{1}{g_{m}}\right)^{2}
$$

and shows that a small $R s$, a large $R d$ and a large $g_{m}$ for the input transistors M1 and M2 should be used. Because the input signal range extends below ground voltage, the last two demands are contradictory. A large $g_{m}$ can be obtained by operating $M 1$ and $M 2$ at large current in weak inversion, which will reduce the voltage span available for a large $R d$. Reducing the Ibias current and operating $M 1$ and $M 2$ in strong inversion is preferred in order to increase the available drain-source voltage on the input devices for linearity reasons. A small $R s$ requires large integration capacitors, thus increasing chip area and power consumption.

Balancing all these constraints and by using a circuit simulator the following optimal values are found for the resistors

$$
\begin{aligned}
& R_{S}=16 k \Omega \\
& R_{D}=36 k \Omega
\end{aligned}
$$

Transistors $M 1$ and $M 2$ are sized with $W=120 \mu m$ and $L=10 \mu m$ to maximize their $r_{d s}$ and $V_{G S}$ (and $V_{D S}$ implicitly) while still keeping their $g_{m}=0.12 \mathrm{mS}$ when Ibias $=15 \mu \mathrm{A}$. All these values yield a total noise power of $25 p \mathrm{~W}$, leaving enough noise margin to the total noise budget of

$$
\frac{V_{i n \_\max }^{2}}{2 D R}=31 p W
$$

to accommodate the reference noise. Opamp noise is of less importance. Due to large bandwidth requirement, the first stage of the amplifier will have a relatively large $g_{m}$. The gain of the input stage of the integrator is also larger than unity. Simulated noise power for the entire circuit, except the $\Sigma \Delta$ feedback sources, is $23 p W$.

The integrating capacitors result from the values of $R s, T_{c l k}$ and $a_{1}$ (Fig. $6.4)$

$$
C i=40 p F
$$

Feedback currents should produce an equivalent feedback voltage of

$$
V_{f b}=f_{1} a_{1} V_{\text {ref }}=265 \mathrm{mV}
$$

on the two Rs resistors. Because only half the clock period is used for feedback leaving the other one for return-to-zero, the current should be doubled

$$
I f b=\frac{2 V_{f b}}{2 R_{S}}=16.5 \mu \mathrm{A}
$$


Total current consumption for the first integrator is $480 \mu \mathrm{A}$, most of it biasing the operational amplifier to reach a unity-gain bandwidth of $6 \mathrm{MHz}$ when loaded by the integration capacitors in series with $R s$ resistors.

The feedback current sources are cascoded PMOS and NMOS devices. The tail current source which keeps the biasing of the two input transistors signalindependent is also cascoded. The switching scheme for the $\Sigma \Delta$ feedback control switches includes digital gates and charge pump voltage doublers. The latter are needed because the switch source is close to the middle of the supply range, leaving approximately one threshold voltage to the supply rail.

\subsubsection{Switched-Capacitor Integrators}

The higher-order integrators are fully differential SC circuits, following the topology shown in Fig. 6.10. They consist of a signal integration path built with the sampling capacitors $C s$, and a $\Sigma \Delta$ feedback integration path represented by $C f b$ capacitors. All integrators deliver their outputs to the adder in front of the quantizer to implement the $b_{1} \cdots b_{3}$ coefficients (Fig. 6.4).

The SC integrators are better suited for low-voltage operation than CT circuits. One advantage is the decoupling of the input signal common-mode Vcmi and the operational amplifier common-mode input Vcmo. As shown in Fig. 6.10, during the sampling phase the switches marked $s$ are closed and the input signal common-mode voltage is Vcmi. During the opposite clock phase, when the switches marked $s$ are open and the other four switches are closed, the common-mode voltage $V c m o$ is used to correctly bias the input stage of the operational amplifier. The two values, Vcmi and Vcmo, are independently set with $V c m i$ tied to the middle of the supply voltage to take advantage of the rail-to-rail output capability of the previous integrator while Vcmo has a low

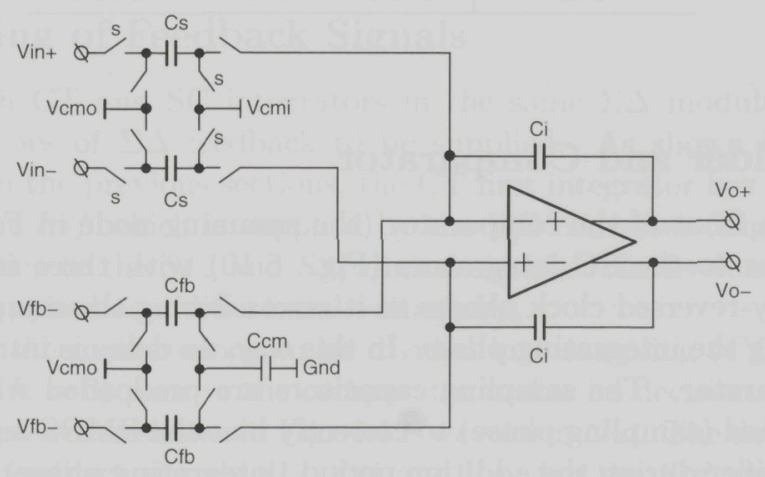

Figure 6.10: Switched-capacitor non-inverting integrator with $\Sigma \Delta$ feedback path 
value falling inside the common-mode input range of the PMOS-input amplifier.

Another advantage of SC circuits is the good control of capacitor ratios. For a CT circuit, a path gain ( $\Sigma \Delta$ loop coefficient) is dependent on the $R C$ or $g_{m} C$ product, which has a typical $3-\sigma$ spread of $30 \%$. In the case of SC circuits the gains are given by a $\mathrm{Cs} / \mathrm{Ci}$ ratio with a spread of less than $1 \%$. In a $\Sigma \Delta$ modulator, the better control of each path gain means that the output range of the integrators in non-overloaded condition can be extended closer to the supply rails without decreasing the yield. The larger the output range, the smaller the capacitive load of the operational amplifier, hence the lower the power consumption.

The MOS capacitor $\mathrm{Ccm}$ is charged to the common-mode value of the differential feedback voltage $V f b+-V f b$-, eliminating the need for an additional buffer circuit.

All the non-overlapping clock phases are generated on-chip. The switching signals for all the SC circuits are boosted by a single set of charge-pump voltage doublers. The second integrator contains a two-stage rail-to-rail output amplifier which attains higher output range compared to the folded-cascode amplifiers used inside the third and fourth integrators. The capacitor values for the higher-order integrators are scaled down considering respective loop gains. The values of sampling, integrating and feedback capacitors are shown in Table 6.1. The sizes of the third and fourth integration capacitors are mismatch-limited, as opposed to the ones in the second integrator which are noise-limited.

Table 6.1: 2nd, 3rd and 4th Integrator Capacitors

\begin{tabular}{c|ccc}
\hline Integrator & $C_{s}[p F]$ & $C_{i}[p F]$ & $C_{f b}[p F]$ \\
\hline 2nd & 0.36 & 1.65 & 0.60 \\
\hline 3rd & 0.20 & 0.40 & 0.24 \\
\hline 4th & 0.30 & 0.60 & 0.24 \\
\hline
\end{tabular}

\subsubsection{Adder and Comparator}

The adder in front of the comparator (the summing node in Fig. 6.4) is a SC circuit similar to the SC integrators (Fig. 6.10) with three input paths and with partially-reversed clock phases so it resets during the sampling phase and it adds during the integrating phase. In this way, no delay is introduced in front of the comparator. The sampling capacitors are pre-loaded with $0.5 \mathrm{~V}$ during the reset period (sampling phase) to correctly bias the PMOS input stage in the adder's amplifier during the addition period (integrating phase) when the input common-mode voltage is half the supply voltage, $0.9 \mathrm{~V}$. The capacitor values are mismatch-limited and are similar to the ones used in the fourth integrator. 
The comparator (Fig. 6.11) is a dynamic circuit, which is pre-charged at $V d d$ during the integrating phase and is allowed to make a decision at the beginning of the sampling phase, when the input voltage (supplied by the adder) is kept stable. Because the adder resets during this phase, advantage is taken of the existence of different clock phases to give approximately $20 \mathrm{~ns}$ of stable input to the comparator. During pre-charge, the RS latch implemented with NOR gates memorizes the previous decision, so no flip-flop is needed after the comparator.

The input transistors $M 1$ and $M 2$ are connected with the sources directly to the ground because the input common-mode voltage is comparable with the MOS threshold voltage. The two NMOS switches controlled by the inverted $C L K$ signal are used to cut the current paths from Vdd to Vss during precharge because no other control of drain current is possible for this half clock period.

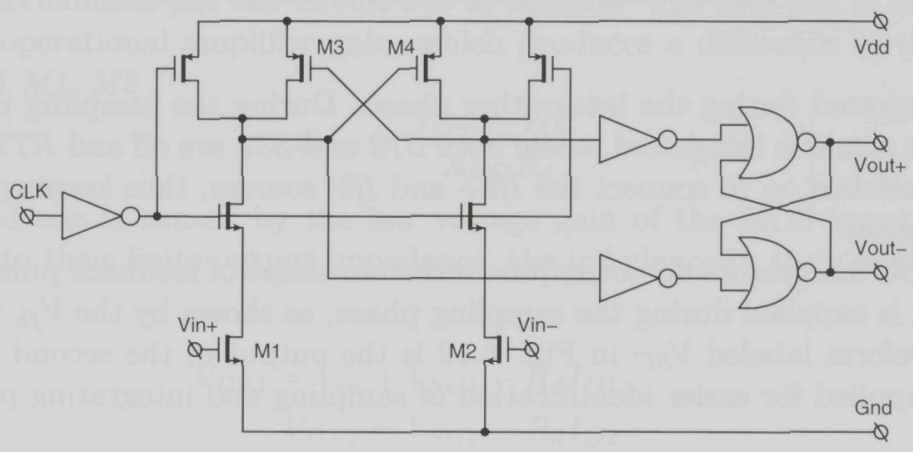

Figure 6.11: Schematic of the dynamic comparator

\subsubsection{Timing of Feedback Signals}

The use of both CT and SC integrators in the same $\Sigma \Delta$ modulator requires two different types of $\Sigma \Delta$ feedback to be supplied. As shown in Fig. 6.12 and explained in the previous sections, the CT first integrator has a differential reference current Iref which is supplied by the V/I converter (Fig. 6.9) and is switched according to the $S 12$ and $S 34$ digital signals. The feedback for the SC integrators is supplied as a differential voltage $V_{f b}$.

Considering the master clock to be divided into sampling $(C L K=1)$ and integrating $(C L K=0)$ phases, the comparator makes its decision at the end of the integrating phase, as shown in Fig. 6.12, waveform Out. The decision is used to generate the feedback signals $S 12, S 34$ and $V_{f b}$. For the S12 and S34 digital signals, the sampling clock is used as return-to-zero control to reduce intersymbol interference [3]. As shown by the waveform $V_{C T}$, the current feedback is 

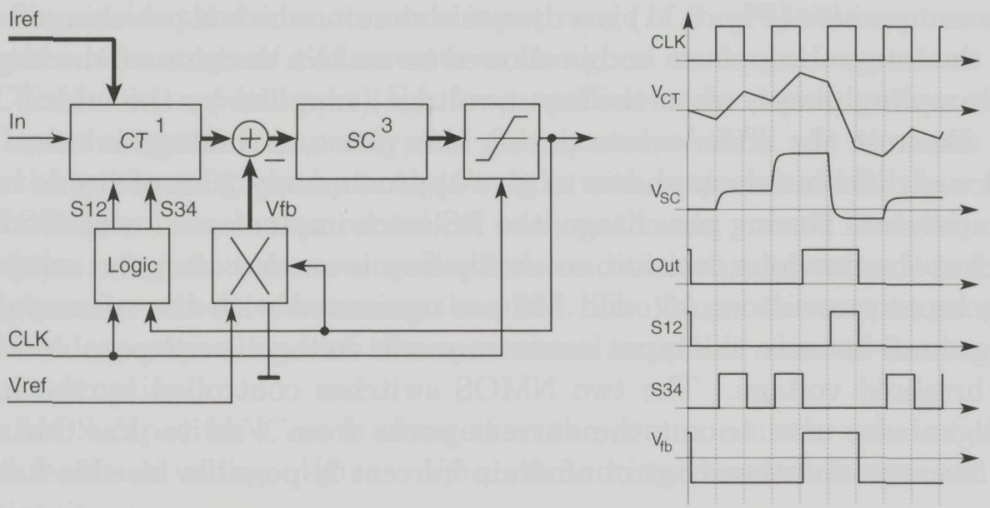

Figure 6.12: Timing of $\Sigma \Delta$ feedback signals

only integrated during the integrating phase. During the sampling phase only the input signal is integrated, while both $S 12$ and $S 34$ are off and $R T Z_{-} c l k$ (Fig. 6.8 ) is switched on to connect the $I f b+$ and $I f b$ - sources, thus keeping them out of saturation.

The SC integrators do not require a certain shape of feedback pulse, so their feedback is supplied during the sampling phase, as shown by the $V_{f b}$ waveform. The waveform labeled $V_{S C}$ in Fig. 6.12 is the output of the second integrator and is supplied for easier identification of sampling and integrating phases.

\subsection{Linearity Analysis}

High linearity can be achieved in single-bit $\Sigma \Delta$ modulators due to the inherent linearity of the 1-bit DAC [5]. The linearity limit in such modulators is the linearity of the integrators, and especially of the first integrator when high OSR values are used. At high OSR, the loop gain offered by the first integrator greatly relaxes the specifications for the rest of the integrators.

The high input impedance integrator used in this design makes use of a capacitive feedback connection to the sources of the MOS transistors M1, M2 (Fig. 6.6) which causes all the signal current to flow directly into the capacitors without passing through the input transistors. Compared to an open-loop MOS pair [3], the input transistors in this circuit are only used as error amplifiers therefore their nonlinearity is reduced by the loop gain. With a differential input signal in the order of $100 \mathrm{mV}$ the integration linearity can reach $100 \mathrm{~dB}$ (better than 16 bits) without requiring high current levels (large transconductance) for the input transistors.

With this topology, the linearity performance is limited by the single-ended- 
to-differential conversion. Imbalance of the input stage allows the commonmode component of the input signal to be converted to a differential output. The resulting common-mode to differential cross talk is

$$
\frac{1}{H}=\frac{\Delta \mu}{\mu} \frac{2 R_{s} g_{d s}}{1+g_{m} R_{s}}
$$

where $\Delta \mu / \mu$ is the relative imbalance of the voltage gain for the MOS transistors

$$
\mu=\frac{g_{m}}{g_{d s}}
$$

These equations assume that the integrator is placed in a feedback loop which helps to keep the output voltage stable. This is a good approximation of a high-oversampling $\Sigma \Delta$ feedback.

Two mechanisms put the circuit out of balance. The first one is caused by the finite operational amplifier gain, which produces a difference in the drain currents of $M 1, M 2$

$$
\Delta I_{D}=\frac{V_{O+}-V_{O-}}{A_{D C} R_{d}}
$$

The second one is caused by the low voltage gain of the MOS input transistors. Due to their finite output impedance, the imbalance in their drain-source voltages

$$
\begin{array}{r}
V_{D S 1}=V_{i n}+V_{S G 1}-R_{d} I_{D 1} \\
V_{D S 2}=V_{S G 2}-R_{d} I_{D 2}
\end{array}
$$

induces a difference in the gate-source voltages. The complete drain current equation of a PMOS transistor in strong inversion

$$
I_{D}=\beta\left(V_{S G}+V_{T H}\right)^{2}\left(1+\lambda V_{S D}\right)
$$

must be used to calculate the two $V_{S G}$ values numerically considering the values of $V_{S D}$ and the equality of the two drain currents. With all the voltages calculated, the expressions of $g_{m}$ and $g_{d s}$ can be derived and used to calculate equation (6.11). An equivalent distorted input signal can be written

$$
V_{i n}^{D I S T}=V_{i n}\left(1+\frac{1}{2 H}\right)
$$

The distortion is generated by the nonlinear dependence of $1 / H$ to the input signal.

For high-accuracy simulations both effects must be taken into account. In this design, the second effect was found to dominate due to the high voltage gain of the amplifier and low output impedance of the MOS transistors. This has 
been analyzed by implementing the equations above in a system-level simulator. Using transistor parameters extracted from technology information, spectra of the modulator output have been simulated to evaluate the design trade-offs. Fig. 6.13 shows such a result, which has been simulated for the circuit parameters used in the final design. The simulated distortion is only $2 \mathrm{~dB}$ lower than the measured one, showing the good accuracy of the simulation. The difference is coming from the unaccounted nonlinear variation of MOST output impedance with its drain-source voltage and shows that the final linearity limit of this circuit is given by the technology used. Therefore, the input MOS transistors are operated between moderate and strong inversion to increase their output impedance and source-to-drain voltage while keeping a high transconductance at low bias current. No cascoding of the input transistors is possible because the input range has to extend below ground.

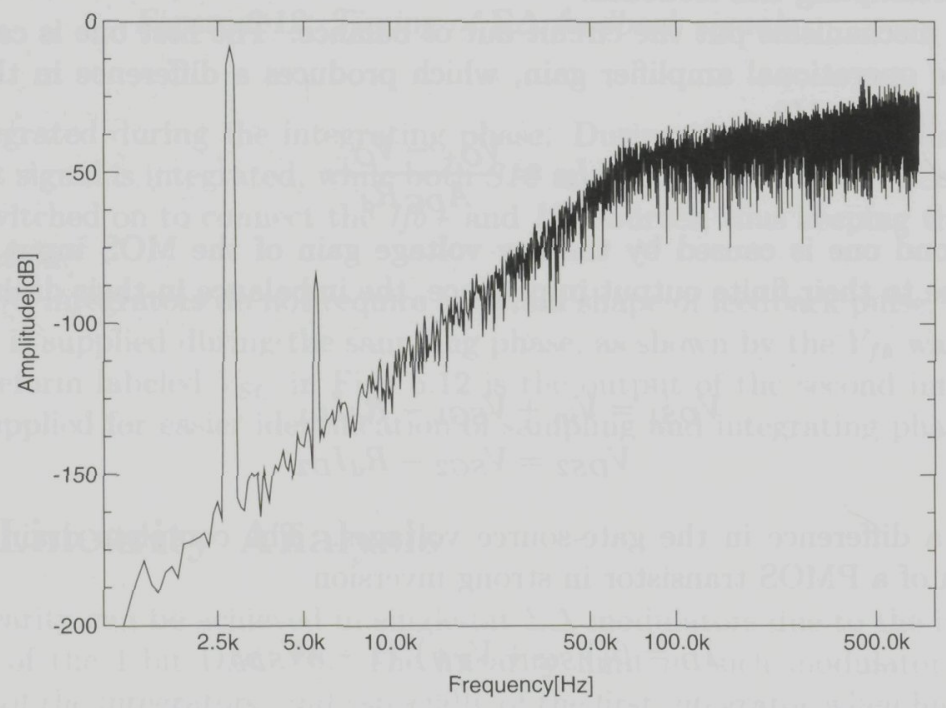

Figure 6.13: FFT of simulated modulator output with full-scale input signal and nonlinear input stage

\subsection{Experimental Results}

The chip has been realized in a $0.5 \mu \mathrm{m}$ CMOS process with a high-linearity capacitor option. A micro-photograph of the layout is shown in Fig. 6.14.

The $\Sigma \Delta$ modulator occupies $0.8 \mathrm{~mm}^{2}$, most of the area being taken by the first integrator capacitors with a total value of $80 \mathrm{pF}$. The on-chip bandgap reference takes another $0.4 \mathrm{~mm}^{2}$. The layout of the bandgap reference has not 


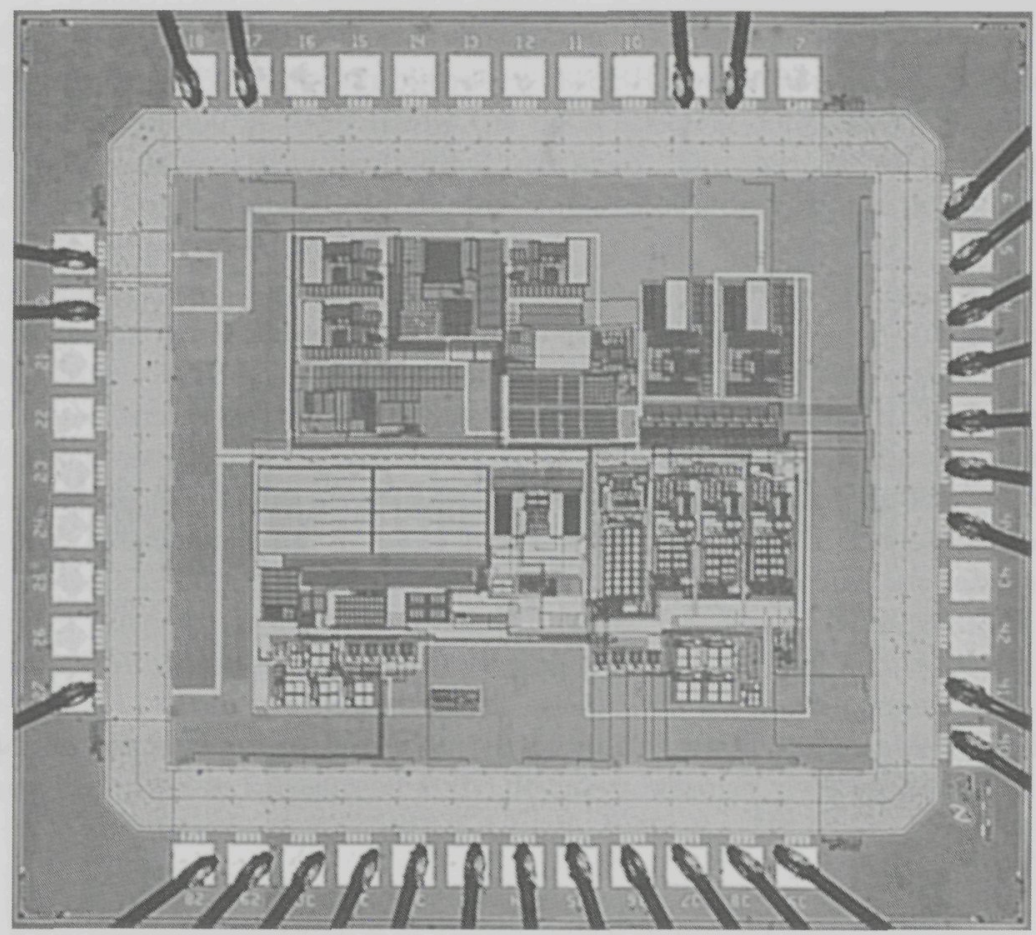

Figure 6.14: Chip micrograph of the $\Sigma \Delta$ modulator with on-chip reference

been optimized for area. Test circuitry is integrated on-chip to allow control of clock lines and observation of integrator outputs. The prototype can be operated in test mode or as a stand-alone acquisition system in which case only five pins are used. An on-chip $5 \mathrm{nA}$ current source is used to bias the input in the modulator to close a DC path from supply to the input node which would otherwise only connect the microphone's capacitive output to modulator's capacitive input. The $5 \mathrm{nA}$ current is derived from a $10 \mu \mathrm{A}$ reference current by a chain of current mirrors. Accuracy of the supplied current is not an issue as long as the current stays below $10 \mathrm{nA}$ (equivalent of a resistor of $180 \mathrm{M} \Omega$ connecting the input node to the supply rail).

All measurements reported here have been taken with the chip biased at $1.8 \mathrm{~V}$ and clocked at $1.404 \mathrm{MHz}$. A single-ended signal generator with 18-bit linearity and noise performance has been attached to the input of the $\Sigma \Delta$ modulator. The small-amplitude single-ended signal requires special care concerning the shielding of signal cables and PCB traces. Fig. 6.15 shows the measured output spectrum when a $2.75 \mathrm{kHz}$, zero DC offset sinusoid is applied at the input. The amplitude of the input signal is $125 \mathrm{mV}(-24 \mathrm{dBR} \mathrm{rms}$ with respect to the design 
Sigma-Delta ADC for Audio Applications

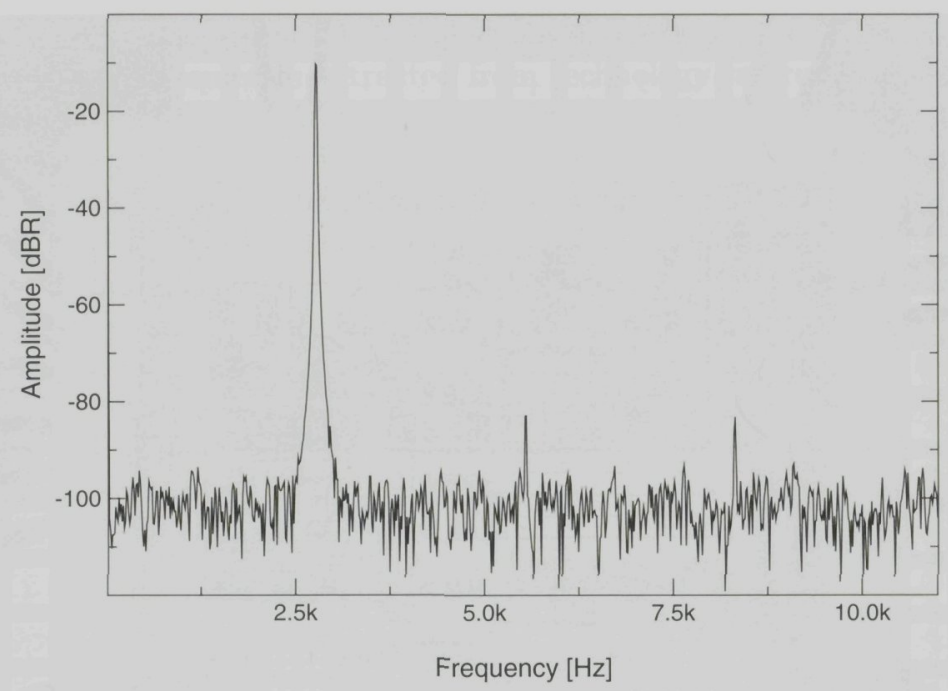

Figure 6.15: Measured FFT of output bit stream with an input signal of -24dBR rms

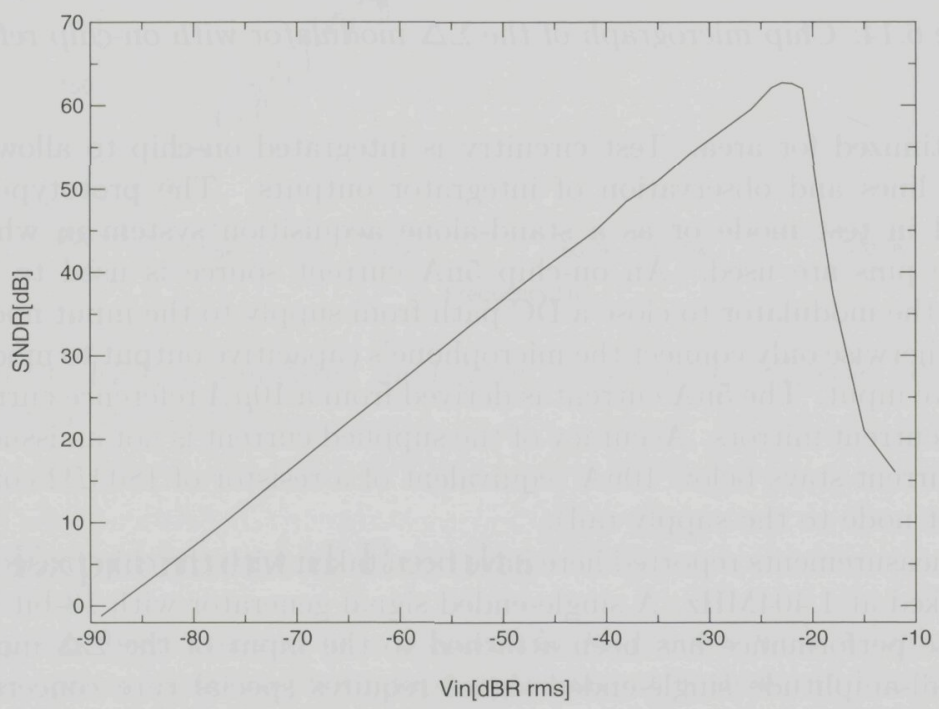

Figure 6.16: Measured SNDR vs input signal level for a chip using internal clocks and references

148 Systematic Design of $\Sigma \Delta$ ADCs 
reference of $1.5 \mathrm{~V}$ ). The spectrum has a frequency resolution (FFT bin) of $14 \mathrm{~Hz}$.

With the -21dBR input amplitude, a -11dBR output is reached. The second harmonic distortion is $73 \mathrm{~dB}$ below the main spectral component, as the theoretical model presented in Section 6.4 predicted. The third harmonic distortion is generated by nonlinearity of the first integrator's operational amplifier gain, which in turn is caused by its large output voltage swing.

The variation of signal-to-(noise+distortion) (SNDR) with input signal amplitude has been measured. The curve is shown in Fig. 6.16 for an input signal ranging from $-90 \mathrm{dBR}$ rms up to $-12 \mathrm{dBR}$ rms. Due to the conversion gain, the upper limit translates to $1 \mathrm{dBR}$ output signal, which is used to test the loop overloading condition. SNDR shows good linearity up to an input signal amplitude of $-23 \mathrm{dBR}$ rms (overloading level). There is a flat region caused by harmonic distortion and, at large input levels, the SNDR sharply drops due to loop overloading. The loop overloads but does not become unstable as the integrator outputs are clipped to supply rails. The peak SNDR value is $62 \mathrm{~dB}$ (10 bits). The measured dynamic range on the $11 \mathrm{kHz}$ signal bandwidth is $80 \mathrm{~dB}$ (more than 13 bits) in a measurement setup with no input connections, made to reduce external noise coming in the circuit through the single-ended input. In the setup used to measure the SNDR curve however, DR drops to $75 \mathrm{~dB}$ be-

Table 6.2: Performance Summary

\begin{tabular}{|c|c|}
\hline Supply Voltage & $1.8 \mathrm{~V}$ \\
\hline$\Sigma \Delta$ Supply Current & $600 \mu \mathrm{A}$ \\
\hline 1st Integrator Supply Current & $480 \mu A$ \\
\hline 2nd Integrator Supply Current & $60 \mu A$ \\
\hline 3rd Integrator Supply Current & $20 \mu A$ \\
\hline 4th Integrator Supply Current & $20 \mu A$ \\
\hline Adder Supply Current & $20 \mu A$ \\
\hline Reference Supply Current & $350 \mu \mathrm{A}$ \\
\hline Total Power Consumption & $1.7 \mathrm{~mW}$ \\
\hline Signal Bandwidth & $11 \mathrm{kHz}$ \\
\hline OSR & 64 \\
\hline Maximum Input Signal & $125 \mathrm{mV}$ peak \\
\hline Dynamic Range & $80 \mathrm{~dB}$ \\
\hline Peak SNDR & $62 \mathrm{~dB}$ \\
\hline Technology & $0.5 \mu \mathrm{m}$ CMOS 2P2M \\
\hline Total Die Area & $1.2 \mathrm{~mm}^{2}$ \\
\hline$\Sigma \Delta$ Die Area & $0.8 \mathrm{~mm}^{2}$ \\
\hline Reference Die Area & $0.4 \mathrm{~mm}^{2}$ \\
\hline
\end{tabular}


Sigma-Delta ADC for Audio Applications

cause of external noise associated with different wires and connectors. Thermal noise dominates the in-band quantization noise. The measured performance and characteristics have been summarized in Table 6.2. 


\section{References}

[1] J.C. Baumhauer et. al, Electroacoustic Transducer with Springs Forming Electrical Interconnections as a Result of Assembly, US Pat. 4046974, Sept. 1977.

[2] J.H. Huijsing, Operational Amplifiers. Theory and Design, Kluwer Academic Publishers, Boston, 2001.

[3] E.J. van der Zwan, E.C. Dijkmans, A 0.2- $m W$ CMOS $\Sigma \Delta$ modulator for Speech Coding with 80 dB Dynamic Range, IEEE Journal of Solid-State Circuits, Vol. 31, pp. 1873, December 1996.

[4] S. Rabii, B.A. Wooley, The Design of Low-Voltage, Low-Power Sigma-Delta Modulators, Kluwer Academic Publishers, Boston, 1999.

[5] S.R. Norsworthy, R. Schreier, G.C. Temes, Delta-Sigma Data Converters, IEEE Press, 1997.

[6] A.L. Coban, P.E. Allen, A $1.5 \mathrm{~V} 1.0 \mathrm{~mW}$ Audio $\mathrm{\Sigma} \Delta$ Modulator with $98 \mathrm{~dB}$ Dynamic Range, Digest of Technical Papers of Intl. Solid-State Circuits Conference, pp. 50, Feb. 1999.

[7] L. Breems, E.J. van der Zwan, J.H. Huijsing, A 1.8-mW CMOS $\Sigma \Delta$ Modulator with Integrated Mixer for A/D Conversion of IF Signals, IEEE Journal of Solid-State Circuits, Vol. 35, pp. 468, Apr. 2000. 
REFERENCES

152 Systematic Design of $\Sigma \Delta$ ADCs 


\section{7 \\ Broadband, High Dynamic Range Sigma-Delta ADC}

The increasing demand for high-speed data links for both mobile and wired applications has spawned ever faster communication protocols which require new architectures and circuits to fulfill the performance requirements (speed, error rate) and keep power consumption and cost low enough to be attractive as consumer applications. One such protocol is Digital Subscriber Line (DSL) which is designed to enable transmission data rates in the Mbit/s range while making use of the existing plain old telephone service (POTS) lines originally designed for a bandwidth as small as $7 \mathrm{kHz}$.

The block schematic of a typical DSL transceiver is shown in Fig. 7.1. Most of the signal processing, from modulation/demodulation to raw data input/output is handled by the DSP, so the entire system is flexible and easy to re-configure, assuming the analog components provide enough SNR and bandwidth. The outbound bit streams are fed into the $D A C$ by the $D S P$. Because the DSL protocols use multicarrier modulation (by splitting the entire bandwidth available in a number of narrow channels), an image rejection filter is used to attenuate the sampled signal's spectral components at multiples of the sampling frequency (images) so they do not interfere with the other channels. In the case of frequency-division duplexing (FDD) or frequency-division multiplexing (FDM) signals, a band-splitting transmit filter is used (Tx filter). In the case of EC systems there is no Tx filter filter in the transmit (Tx) path. Instead, digital echo cancellers are implemented by the DSP to subtract the Tx signal from the received signal $(\mathrm{Rx})$. Finally, a line driver is used to drive the line impedance which is $100 \Omega$ in US and $135 \Omega$ in Europe.

The receive path begins with the $\mathrm{Rx}$ amplifier $(R x A m p)$ which is a difference amplifier with programmable gain. An Rx filter is required in FDD or FDM 


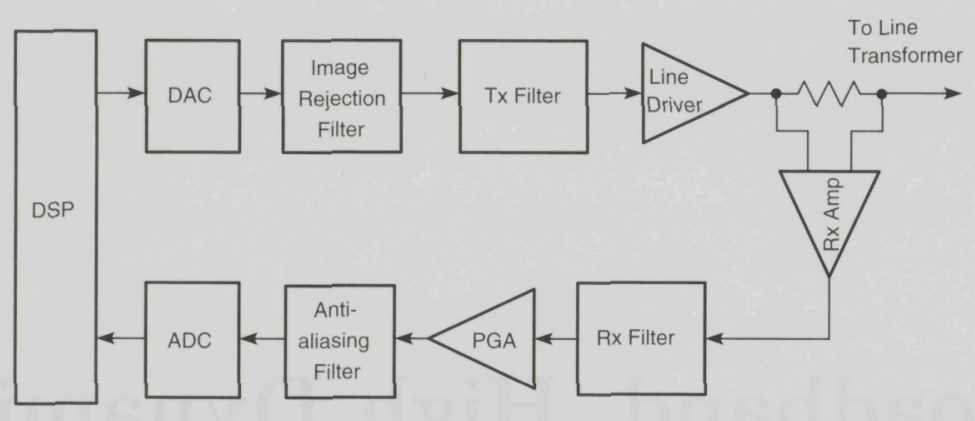

Figure 7.1: Block schematic of a DSL transceiver

systems and can also be employed with EC systems if the Tx bandwidth is larger than the Rx bandwidth. The programmable gain amplifier $P G A$ is needed to adapt the input signal range to that of the ADC after the signal is passed through the anti-aliasing filter.

Because DSL systems perform the modulation/demodulation in the digital domain, the ADC and the DAC have to process not only a single, narrowbandwidth channel but the entire communications bandwidth. This is the concept of the "software radio" applied at much lower frequencies than RF communications. Total bandwidth for ADSL, the most popular DSL protocol, is $1.1 \mathrm{MHz}[1]$. But the most aggressive member of the family, VDSL, can extend up to $12 \mathrm{MHz}$ bandwidth. On the Rx path this means the ADC should be able to convert a bandwidth in the order of 1 to $10 \mathrm{MHz}$ with a resolution which is high enough to guarantee a certain bit error rate (BER), as specified by communication standards. Increasing the resolution of the ADC can simplify the overall system by softening requirements imposed on analog filtering due to increased tolerance to interferers. In the same time, a better use of channel capacity can be obtained by making the channel noise the dominant source of error.

This Chapter describes in detail the design of a high-resolution, broadband $\Sigma \Delta$ ADC. The design starts from a top-level specification extracted from performance requirements of the DSL transceiver chain. From this specification and taking into account technology constraints, an optimal solution is found using the CAD programs covered in Chapter 5. The design is then implemented at transistor-level and, at this level, Spice-like simulations are performed to measure the final power consumption and performance in terms of noise and linearity. In the following Section, the performance requirements of the $\Sigma \Delta$ $\mathrm{ADC}$ are calculated from the DSL channel performance. In Section 7.2, an optimal solution is found based on performance, technology constraints and other

\section{Systematic Design of $\Sigma \Delta$ ADCs}




\subsection{Project Specifications}

constraints imposed by the designer. The building blocks of the $\Sigma \Delta$ ADC are then presented in detail, at transistor-level, in the Sections 7.3, 7.4, 7.5 and 7.6. Section 7.7 covers the calibration procedure used to linearize the first DAC in the $\Sigma \Delta$ loop. Transistor-level simulation results are presented in Section 7.8.

\subsection{Project Specifications}

Depending on the modulation scheme, the required ADC resolution varies differently with desired data rate for a given channel bandwidth. One of the simplest baseband modulation schemes, pulse amplitude modulation (PAM), requires mapping of $M=2^{b}$ codes (referred of as b-bit PAM or M-ary PAR) as equally-spaced levels around zero. In this case, the required SNR for the ADC is

$$
S N R=20 \log \left(Q^{-1}\left(\frac{1}{2(1-1 / M)}\right) P_{P A M}\right)+20 \log (M-1)+20 \log (\beta)-3
$$

with $Q(x)$ being the probability that a Gaussian random variable of unit variance is larger than $x, P_{P A M}$ is the tolerated error probability and $\beta$ is the excess bandwidth (ratio of channel bandwidth and symbol rate, typically 2). With this formula it can be calculated that the coding of 256 levels $(M=256)$ requires 11 bits resolution in the ADC, and the required resolution doubles with the doubling of $M$.

For a widely-used passband modulation scheme, quadrature amplitude modulation (QAM), two carriers in quadrature are used to transmit 2 bits in the same time. In this case, written using the same symbols as in Eq. 7.1, the SNR is given by

$$
\begin{aligned}
S N R= & 20 \log \left(Q^{-1}\left(\frac{1}{4(1-1 / \sqrt{M})}\right) P_{Q A M}\right) \\
& +10 \log \left(\frac{(M-1)(\sqrt{M}-1)}{\sqrt{M+1}}\right) \\
& +20 \log (M-1)+20 \log (\beta)
\end{aligned}
$$

The number of required bits of resolution in the ADC is no longer proportional with the number of coded bits, only 7 bits of resolution being required for $M=256$.

The QAM modulation is also used for DMT modulations like ADSL. In this case, up to 255 carriers are equally-spaced in the conversion bandwidth, each of them being used as one QAM carrier. Because more carriers are simultaneously converted, the ADC has to accommodate larger peak signals (worst case is when all carriers are in phase) so the required SNR doubles when the number of carriers is multiplied by 4 . The excess bandwidth $\beta$ is 1 (all bandwidth is 
occupied by the carriers) so the SNR formula for DMT systems using QAM is obtained from Eq. 7.2 by replacing $\beta$ with the number of carriers. With 255 carriers (ADSL), a resolution of 11 bits is required for $M=256$.

The requirements on the ADC performance are dramatically increased, however, when no filtering is available and the ADC connects directly to the line signal. Without filtering, the strong echo signal from the transmit path is also converted and should be accommodated by the ADC. The line noise also plays an important role, as it sets the absolute limit for the channel capacity at a given symbol rate. Considering all signal and noise sources, the ADC's SNR is given by

$$
S N R=P_{\text {signal }}-P_{\text {noise }}+20 \log \left(\frac{P A R}{1.4}\right)
$$

with $P A R$ being the peak-to-average ratio of the input signal. Here, $P A R$ expresses the number of sinusoids with equal amplitude converted in the same time and increases the SNR by one bit for every doubling of sinusoids number. With Tx echo considered input signal as well, and knowing the SNR requirements for echo-free QAM from Eq. 7.2, Eq. 7.3 can be expanded as

$$
S N R=P S D_{\text {signal }}-P S D_{n}-10 \log \left(B W_{R x}\right)+20 \log \left(\frac{P A R}{1.4}\right)
$$

with $P S D_{\text {signal }}$ given by

$$
\begin{aligned}
10^{\left(P S D_{\text {signal }} / 10\right)}= & 10^{\left(P S D_{T x}-T_{e}+10 \log \left(B W_{T x}\right)\right) / 10} \\
& +10^{\left(P S D_{n}+S N R_{Q A M}+10 \log \left(B W_{R x}\right)\right) / 10}
\end{aligned}
$$

where $P S D$ denotes a power spectral distribution, $B W$ denotes a signal bandwidth, $T x$ and $R x$ indices stand for transmit and receive properties while $n$ index stands for line noise properties. $T_{e}$ measures the echo rejection available. For extremely short lines $T_{e}$ is $0 \mathrm{~dB}$, and it can increase with line length up to $20 \mathrm{~dB}$. For a 255 -carriers ADSL, 32768-QAM (15 bit), $B W_{R x}=1.078 \mathrm{MHz}$, error probability $10^{-7}$, and $0 \mathrm{~dB}$ echo rejection in the presence of a $-140 \mathrm{dBm} / \mathrm{Hz}$ line noise, the ADC resolution should be as high as 20 bits $(120 \mathrm{~dB})$. In the presence of higher line noise and with $20 \mathrm{~dB}$ echo rejection the required resolution drops to 12 bits.

To reduce assembly and testing costs, the ADC described in this chapter is designed to operate in a system with no echo rejection filters, so $T_{e}=0$. The line noise is considered to be $-120 \mathrm{dBm} / \mathrm{Hz}$ and the protocol is ADSL, 32768-QAM, error rate $P_{Q A M}=10^{-7}, B W_{R x}=1.078 M H z$. Under these assumptions, the required resolution is more than 16 bits $(96 \mathrm{~dB})$. The $\mathrm{ADC}$ is a $\Sigma \Delta$ modulator designed to attain $96 \mathrm{~dB}$ of resolution over a bandwidth of $2 \mathrm{MHz}$, which is a factor $1.855(2.7 \mathrm{~dB})$ larger than $B W_{R x}$. This will give additional oversampling 
ratio to the conversion, raising the resolution in the band of interest by 0.5 bit. The $2 \mathrm{MHz}$ bandwidth is more convenient because it is oversampled by the $\Sigma \Delta$ ADC, usually by a power-of-2 OSR, and the resulting sampling frequency should match one of the commercially-available low-jitter crystal oscillators.

The ADC does not need a high input impedance because the PGA (Fig. 7.1) is always present in the Rx path to adjust input signal to full ADC input range. Therefore, both $\mathrm{SC}$ and $\mathrm{CT}$ designs for the loop filter can be considered. The DAC inside the loop is multibit, the only option to increase resolution when OSR is basically limited by technological reasons. To attain 96dB SNR for a fourth order, single bit loop the required OSR is 64 . For $2 \mathrm{MHz}$ conversion bandwidth this translates to a clock frequency of $256 \mathrm{MHz}$, which means the operational amplifier inside the first integrator needs a unity-gain bandwidth (UGB) of approximately $4 \mathrm{GHz}$. Besides the technology and packaging problems, this is also increasing the power consumption of the ADC so is not an attractive solution.

Because the ADC is designed to be placed on the same chip with the DSP, an advanced digital CMOS process is used. At the date this project started the most advanced yet stable CMOS process was the $0.18 \mu \mathrm{m}$, without any analog options. The $0.18 \mu \mathrm{m}$ process offers high-performance MOS transistors to be operated at a maximal supply voltage of $1.8 \mathrm{~V}$, and optionally $3.3 \mathrm{~V} \mathrm{I} / \mathrm{O}$ transistors which are not recommended for high-performance analog circuits.

\subsection{CAD-Assisted Design Optimization}

The CAD software described in Chapter 5 is used to evaluate all possible solutions and select the best one, from the designer's point of view. The design performance requirements, DR (SNDR) and signal bandwidth, are used in conjunction with technology-related constraints, like maximal sampling frequency, to reach an optimal solution by incrementally narrowing the solutions space under designer supervision. The loop topology contains all feedbacks to integrators and all feed-forwards from each integrator to the quantizer input (similar to Fig. 6.4 in Chapter 6).

Both SC and CT solution spaces are searched. The specifications are: target DR $98 \mathrm{~dB}, V_{\text {supply }}=1.8 \mathrm{~V}$, signal bandwidth $2 \mathrm{MHz}$, reference voltage $1.8 \mathrm{~V}$, clipping at $80 \%$ of $V_{\text {supply }}$.

\subsubsection{Directing the CAD Software Toward an Optimal So- lution}

Following the procedure outlined in Chapter 5 and using the optimization software described there, an exhaustive search is conducted to find the best-fit 
solution. First of all, a global search is performed for SC loop filters, in the entire design space, i.e. loop order (ORDER) from 1 to 6 , number of bits (BITS) from 1 to 8 , number of cascaded loops (LOOPS) from 1 to 4 and oversampling ratios $(O S R)$ from 8 to 256 . The results of CAD optimization are shown in Table 7.1, as a list of top ten best solutions from the point of view of a figure of merit $(F O M)$ defined as

$$
F O M=\frac{P e a k S N D R}{P}[d B]
$$

where $P$ is the power consumption required to implement the $g_{m}$ in all the operational amplifiers (see Chapter 5).

For each solution the table shows the order, the number of loops with the list of cascaded loops orders in parentheses, the number of bits in the last loop's quantizer with the number of bits in the first $n-1$ cascaded loops in parentheses, the oversampling ratio, the SNDR and the FOM in $\mathrm{dB}$.

It can be seen that theoretically the best performance is given by highoversampling, low order solutions. The solution with the largest FOM is a first order, 8-bit solution working at 128 OSR. The good performance of loworder, highly-oversampled $\Sigma \Delta$ modulators is caused by the relatively simple model used to evaluate their performance: there is no power penalty for increased number of bits in the quantizer and no SNDR penalty for extremely high sampling frequencies. This is not true in circuit design, where correcting the mismatch in a multibit DAC can cost as much as $40 \%$ of the total power consumption [2] and clocking a $\Sigma \Delta$ modulator at gigahertz frequencies can reduce theoretically-predicted SNDR due to difficulties in designing fast comparators and latches without adding excessive switching noise that can alias inside the signal bandwidth [3].

Table 7.1: Global solutions for Broadband SC ADC

\begin{tabular}{|c|c|c|c|c|c|}
\hline ORDER & LOOPS & BITS & OSR & SNDR & FOM \\
\hline 1 & 1 & 8 & 128 & 99.8 & 105.5 \\
\hline 2 & $2(1-1)$ & $2(5)$ & 256 & 102.7 & 104.4 \\
\hline 2 & $2(1-1)$ & $1(5)$ & 256 & 101.6 & 103.3 \\
\hline 4 & 1 & 2 & 64 & 98 & 102.3 \\
\hline 3 & 1 & 5 & 32 & 97.2 & 102.0 \\
\hline 6 & $2(2-4)$ & $2(6)$ & 32 & 97.6 & 102.0 \\
\hline 3 & 1 & 7 & 16 & 97.5 & 101.8 \\
\hline 4 & $3(2-1-1)$ & $3(7)$ & 16 & 97.1 & 101.6 \\
\hline 6 & $3(2-1-3)$ & $2(7)$ & 32 & 98.3 & 101.6 \\
\hline 6 & $3(2-3-1)$ & $2(7)$ & 16 & 97.5 & 101.6 \\
\hline
\end{tabular}


The solutions with order higher than 3 can offer enough performance while working at OSR 32 but this still means a sampling frequency of $128 \mathrm{MHz}$. To decrease the sampling frequency lower than $100 \mathrm{MHz}$ the third order solution which appears in the table needs seven bits in the quantizer but has a good FOM for a single-loop architecture. The other two solutions with $\mathrm{OSR}=16$ are cascaded architectures, also with 7 bits in the first quantizer. The difference of $1 \mathrm{~dB}$ in peak SNDR does not justify increasing complexity, so the next step is to look in greater detail inside the solutions sub-space obtained by an additional design constraint, $\mathrm{OSR}=16$.

The results of this constrained search are listed in Table 7.2. There are many cascaded solutions, again with large number of bits in the first quantizer. This increased resolution is needed to accommodate coefficient-to-coefficient mismatch. The mismatch is considered to be as high as $1 \%$ because metalmetal capacitors are used and no detailed matching data is available for these devices. Metal-metal capacitors are preferred for their high linearity even if they take a large chip area and the thickness of inter-metal oxide (field oxide) is not very well controlled during processing so spread can be significant.

The sensitivity to coefficient mismatch is a major drawback of cascaded solutions [4] but it becomes less important as technology features decrease and better control of processing parameters is achieved. But smaller-feature CMOS technologies also work at lower supply voltage $(3.3 \mathrm{~V}$ at $0.35 \mu \mathrm{m}$ down to $2.5 \mathrm{~V}$ at $0.25 \mu \mathrm{m}$ and 1.8 for $0.18 \mu \mathrm{m}$ ) and push the threshold voltage of MOS transistors at lower values, which is detrimental for analog designs as this reduces the output impedance of the transistors. Reduction of output impedance is difficult to correct under reduced output voltage swing when cascoding is difficult to employ. For $\Sigma \Delta$ ADC design, all these effects make difficult the design of opamps with high and signal-independent gain. These opamps are needed in

Table 7.2: Broadband SC ADC Solutions with $O S R=16$

\begin{tabular}{|c|c|c|c|c|}
\hline ORDER & LOOPS & BITS & SNDR & FOM \\
\hline 4 & $3(2-1-1)$ & $3(7)$ & 97.6 & 102.1 \\
\hline 4 & $3(2-1-1)$ & $4(7)$ & 97.4 & 101.6 \\
\hline 3 & 1 & 7 & 97.4 & 101.6 \\
\hline 6 & $3(2-2-2)$ & $3(8)$ & 97.7 & 101.6 \\
\hline 6 & $3(2-3-1)$ & $3(7)$ & 97.7 & 101.4 \\
\hline 4 & $3(2-1-1)$ & $5(7)$ & 97.4 & 101.2 \\
\hline 6 & $3(2-2-2)$ & $2(8)$ & 97.2 & 101.2 \\
\hline 5 & $3(2-1-2)$ & $2(8)$ & 96.3 & 100.5 \\
\hline 5 & $3(2-1-2)$ & $3(8)$ & 96.3 & 100.4 \\
\hline 5 & $4(2-1-1-1)$ & $2(7)$ & 96.2 & 100.1 \\
\hline
\end{tabular}


cascaded designs because they guarantee the matching of NTFs implemented in different loops in the cascade, hence reducing the leakage of low-order shaped noise to the output [5].

Therefore, the next step in optimization is to further reduce the design space by an additional constraint, LOOPS $=1$. The single-loop $\Sigma \Delta$ designs are known to be tolerant to both coefficient-to-coefficient mismatch and integrator leakage introduced by low-gain opamps used inside the integrators, because their tolerance to NTF performance shift can be made arbitrarily high. The results of this last optimization step are listed in Table 7.3. The best FOM belongs to the third order, seven-bits solution due to its reduced count of integrators and an SNDR level comparable to the other solutions in the table. Its FOM and SNDR vary slightly from the ones in Table 7.2 because random effects are included in the design process and, for simulation time limiting, the length of random samples is not extremely large so their statistical properties vary slightly from run to run.

There are many fourth-order solutions with five to eight bits in the quantizer, two fifth-order and one sixth-order solutions. Choosing the best solution from the designer's point of view means balancing all the advantages and disadvantages of the solutions listed in Table 7.3 in a process similar to the previous design space reduction steps. The best solution, $(\mathrm{ORDER}=3$, BITS $=7)$, has an extremely large number of bits in the quantizer which would require $2^{7}-1$ comparators and identical DACs in the loop. The best solution from this point of view is the (ORDER $=6$, BITS $=4)$ but it shows a FOM which is twice smaller than for $(\mathrm{ORDER}=3$, BITS=7). This means it burns two times more power for a reduced SNDR so is not a good choice unless the small number of bits is really critical. In the design presented here, a solution with five bits can be implemented (31 comparators and DACs are not extremely critical to implement on silicon in a high-speed process) and the best solution is (ORDER=4,

Table 7.3: Broadband SC ADC Solutions with $O S R=16$, LOOPS $=1$

\begin{tabular}{|c|c|c|c|}
\hline ORDER & BITS & SNDR & FOM \\
\hline 3 & 7 & 97.9 & 102.1 \\
\hline 3 & 8 & 97.5 & 101.4 \\
\hline 4 & 6 & 97.7 & 101.4 \\
\hline 4 & 5 & 97.1 & 101.2 \\
\hline 4 & 7 & 97.7 & 100.8 \\
\hline 4 & 8 & 97.8 & 100.1 \\
\hline 5 & 5 & 97.3 & 100.0 \\
\hline 5 & 7 & 98.1 & 99.5 \\
\hline 6 & 4 & 95.5 & 97.9 \\
\hline
\end{tabular}




\subsection{CAD-Assisted Design Optimization}

BITS=5) which has a FOM only $1 \mathrm{~dB}$ worse than $(\mathrm{ORDER}=3, \mathrm{BITS}=7)$. Its peak SNDR is also comparable to the top solution for a four-times reduced quantizer complexity.

The CT solutions found to be globally optimal are listed in Table 7.4. Compared to the SC solutions there is an abundance of modulators working at $\mathrm{OSR}=16$ which shows that CT solutions are a better choice for high-frequency (low OSR) designs. Their power consumption is much lower then the SC counterparts, as shown by the approximately $3 \mathrm{~dB}$ larger FOM values for comparable SNDRs. However, a larger number of bits in the quantizer or a larger order are needed for the same peak SNDR. This is caused by the reduced overloading levels in CT designs compared to SC designs, as well as the drastic limitations of the design space for CT loop filters, as explained in Chapter 5.

Table 7.4: Global solutions for Broadband CT ADC

\begin{tabular}{|c|c|c|c|c|c|}
\hline ORDER & LOOPS & BITS & OSR & SNDR & FOM \\
\hline 1 & 1 & 7 & 256 & 104.3 & 114.0 \\
\hline 3 & $2(2-1)$ & $8(8)$ & 32 & 98.0 & 105.6 \\
\hline 6 & $3(2-1-3)$ & $5(6)$ & 16 & 97.6 & 105.3 \\
\hline 5 & $3(2-1-2)$ & $5(6)$ & 16 & 96.8 & 105.0 \\
\hline 5 & $3(2-2-1)$ & $5(8)$ & 16 & 96.9 & 104.8 \\
\hline 4 & $3(2-1-1)$ & $6(6)$ & 16 & 96.6 & 104.6 \\
\hline 5 & $4(2-1-1-1)$ & $4(6)$ & 16 & 96.6 & 104.5 \\
\hline 6 & $4(2-1-1-2)$ & $3(7)$ & 16 & 96.4 & 104.3 \\
\hline 6 & $4(2-1-2-1)$ & $3(7)$ & 16 & 96.2 & 104.0 \\
\hline 6 & $4(2-2-1-1)$ & $4(8)$ & 16 & 95.6 & 102.7 \\
\hline
\end{tabular}

For a comparison against the set of SC final solutions with $\mathrm{LOOPS}=1$, $\mathrm{OSR}=16$, an equivalent optimization has been run for the CT solutions. The results are listed in Table 7.5. The least number of bits required is 6 for a

Table 7.5: Broadband CT ADC Solutions with $O S R=16, L O O P S=1$

\begin{tabular}{|c|c|c|c|}
\hline ORDER & BITS & SNDR & FOM \\
\hline 4 & 7 & 95.0 & 103.4 \\
\hline 5 & 8 & 98.2 & 101.3 \\
\hline 4 & 8 & 93.2 & 100.1 \\
\hline 6 & 6 & 90.1 & 96.5 \\
\hline 6 & 7 & 91.0 & 96.5 \\
\hline 5 & 7 & 88.0 & 93.0 \\
\hline 6 & 8 & 85.3 & 89.3 \\
\hline
\end{tabular}


sixth order loop. It is interesting to note that power consumption as reflected by FOM values is better only for certain solutions, not as many as they were in the global solution set. This is caused by the restriction to have a one-loop filter which translates typically in smaller integrator coefficients meaning larger integration capacitors compared to the cascaded designs. The fact that, for a CT integrator, the coefficient is given by an $\mathrm{RC}$ constant with processing spread of $+/-30 \%$ further increases the integration capacitor values through the need for lower coefficients to avoid state clipping.

\subsubsection{Loop Coefficients}

Based on the findings explained in the previous section, the best solution (chosen based not only on the FOM) is the single-loop, fourth order, 5-bit working at $\mathrm{OSR}=16$ (Table 7.3) and designed with SC circuits. The SC circuits allow an aggressive NTF to be used for a given loop order, therefore the number of required bits is kept relatively low without significantly degrading the overloading level. The chosen architecture does not offer the best FOM, but is the best fit for a high-speed CMOS technology.

The loop topology (Fig. 7.2) contains four integrators with separate feedback paths for the 5 -bit DACs $f_{1} \cdots f_{4}$, a 5 -bit quantizer with 31 decision levels (31 comparators) and feedforward paths $b_{1} \cdots b_{3}$ for control of STF characteristics. Values for NTF peak, needed opamp gain in each integrator, and loop coefficients, as output by the CAD software, are:

NTFmax_value $\quad 5.795$

OpAmp_Gain $24.082400 \mathrm{~dB}$

$\mathrm{a}[1]=5.625000 \mathrm{e}-01$

$\mathrm{a}[2]=1.600000 \mathrm{e}+00$

$\mathrm{a}[3]=2.285714 \mathrm{e}+00$

$\mathrm{a}[4]=4.000000 \mathrm{e}-01$

b [1] $=7.647059 e-01$

b [2] $=1.416667 \mathrm{e}+00$

b [3] $=8.125000 \mathrm{e}-01$

$f[1]=-1.000000 e+00$

$f[2]=-8.750000 e-01$

$f[3]=-5.625000 e-01$

$\mathrm{f}[4]=5.000000 \mathrm{e}-01$

As the list shows, the peak NTF magnitude is large (more than 15dB) but still the loop is stable due to a large number of quantizer bits. Values for loop coefficients are convenient, which means they are not extremely low, therefore 


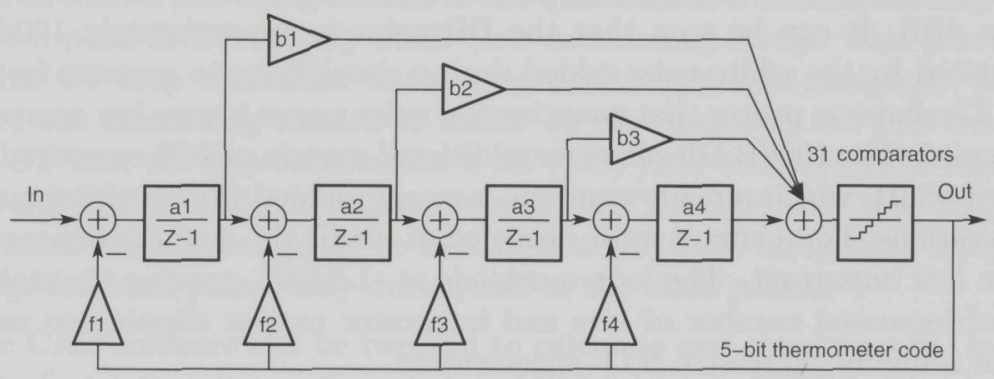

Figure 7.2: Loop topology for the DSL $\Sigma \Delta A D C$

the power consumption can be kept low. The coefficients are designed to bound the integrator outputs to $+/-1.4 \mathrm{~V}$, leaving $0.2 \mathrm{~V}$ margin to both supply rails. Required operational amplifier gain is at least $16(24 \mathrm{~dB})$, a value easy to attain even with a 1-stage amplifier without cascoding of amplifying transistors.

The performance characteristics of the chosen solution can be observed from

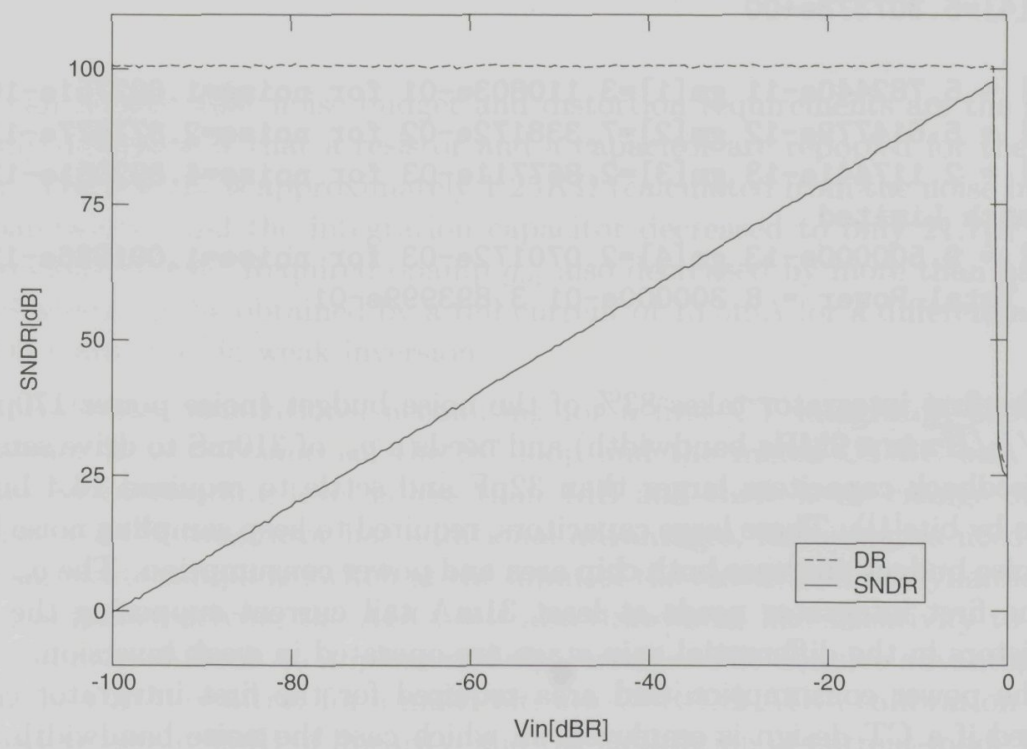

Figure 7.3: Simulated DR and SNDR curves for chosen $\Sigma \Delta A D C$ 


\section{Broadband, High Dynamic Range Sigma-Delta ADC}

Fig. 7.3 which shows simulated DR and SNDR variations with input signal level in $\mathrm{dBR}$. It can be seen that the DR value is approximately $100 \mathrm{~dB}$ and flat, limited by the white noise added during simulations to account for circuit noise. The flatness proves that quantization noise power is very low compared to white noise. The $100 \mathrm{~dB}$ DR offers an additional margin of $2 \mathrm{~dB}$ compared to the targeted $98 \mathrm{~dB}$, which already contains a margin added from architecture-level considerations. For a circuit noise designed at $-98 \mathrm{~dB}$ the quantization noise will be even less important. The loop overloads at $-1.5 \mathrm{dBR}$, proving the stabilizing effect of increased number of bits and integrator output clipping to supplies, even for a highly aggressive NTF specification.

\subsubsection{Power Dissipation for DT and CT Circuits}

The SC loop performs well at the architecture level, but the circuit-level design can still be challenging. The output of the CAD software contains a list of required capacitors and $g_{m}$ opamp transconductances calculated for each integrators from noise, distortion and matching considerations. For the SC loop chosen for this design, the circuit-level output is:

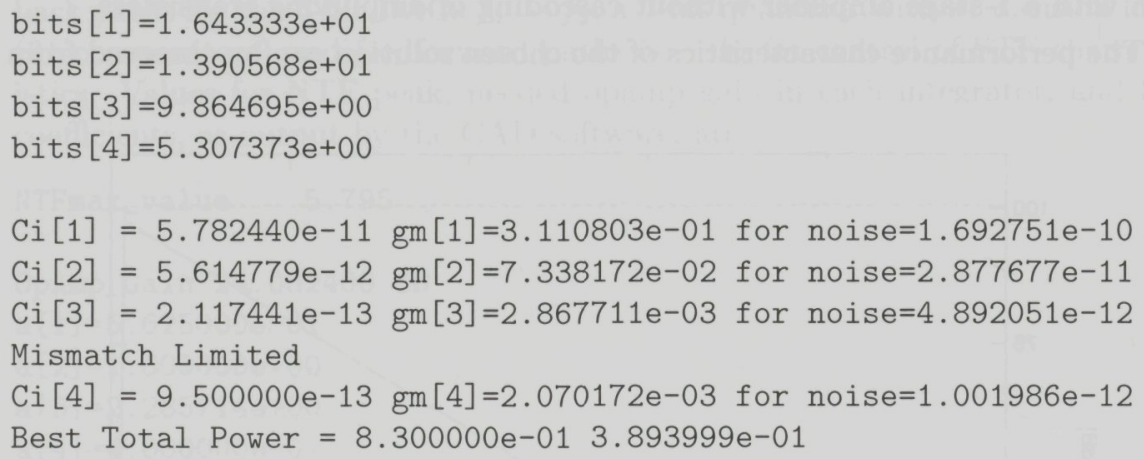

The first integrator takes $83 \%$ of the noise budget (noise power $170 \mathrm{pW}$ or $9.2 n \mathrm{~V} / \sqrt{\mathrm{Hz}}$ in a $2 \mathrm{MHz}$ bandwidth) and needs a $g_{m}$ of $310 \mathrm{mS}$ to drive sampling and feedback capacitors larger than $32 \mathrm{pF}$ and settle to required 16.4 bits (as shown by bits[1]). These large capacitors, required to keep sampling noise below the noise budget, increase both chip area and power consumption. The $g_{m}$ listed for the first integrator needs at least $31 \mathrm{~mA}$ tail current supposing the MOS transistors in the differential gain stage are operated in weak inversion.

The power consumption and area required for the first integrator can be reduced if a CT design is employed, in which case the noise bandwidth is decreasing, hence the size of the integration capacitor. The attempt to generate a CT solution has been explained in the previous sub-section, the result of which 


\subsection{CAD-Assisted Design Optimization}

showed at least 6 bits being needed in the quantizer of a sixth order loop at the price of a peak SNDR of only $90 \mathrm{~dB}$ (low overloading level). This proves that no stable CT loop equivalent to the target $\mathrm{SC}$ one can be designed. There is however one interesting remark to make: by changing only the first integrator into a CT one, the loop behaves like a $S C$ (DT) filter as long as the rest of the loop is $S C$ and the $C T$ first integrator uses a RTZ DAC pulse. The RTZ DAC pulse makes the CT integrator to behave closer to a SC integrator, integrating the large feedback pulses only during half of the clock period.

The CAD software can be tweaked to calculate and simulate a SC loop but map the first integrator coefficients to a CT integrator. For the current design, the output of such a run reads:

bits $[1]=1.643333 \mathrm{e}+01$

bits $[2]=1.390568 \mathrm{e}+01$

bits $[3]=9.864695 \mathrm{e}+00$

bits $[4]=5.307373 e+00$

$\begin{aligned} \operatorname{Ri}[1]=1.275285 e+03 \operatorname{Ci}[1]=2.178162 e-11 & \text { gm }[1]=1.343768 e-01 \\ & \text { for noise }=1.692751 e-10\end{aligned}$

It can be seen that noise budget and distortion requirements are the same, the only difference is that a resistor and a capacitor are reported for the integrator. The resistor is approximately $1.25 K \Omega$ (calculated from the noise budget and bandwidth) and the integration capacitor decreased to only $21.7 \mathrm{pF}$ from the previous $57.8 \mathrm{pF}$ ! Required opamp $g_{m}$ also decreased by more than half, to $135 \mathrm{mS}$ which can be obtained by a tail current of $13.5 \mathrm{~mA}$ for a differential pair of MOS transistors in weak inversion.

Time-domain simulations, accounting for a first CT integrator, show the equivalence in performance of the $\mathrm{SC}$ loop and the mixed $\mathrm{CT}-\mathrm{SC}$ one. The decrease in overloading level is less than $1 \mathrm{~dB}$ and there is no change of DR. The use of a CT integrator has additional advantages, including no need for a highly-accurate sampling switch at the input of the circuit and no dynamic load for the amplifier driving the ADC, but also drawbacks like sensitivity to clock jitter and more difficult to implement DAC circuits. The decisive advantage lies also in the DAC circuitry: for a multi-bit, low-OSR $\Sigma \Delta \mathrm{ADC}$, calibration is the only way to attain $96 \mathrm{~dB}$ of linearity, and the calibration of current-mode DACs in MOS circuits is a better option than the calibration of voltage-mode (SC) DACs. 


\subsection{Design of the 16-bit CT Integrator}

\subsubsection{Integrator Topology}

An active- $\mathrm{RC}$ topology is used for the first integrator (Fig. 7.4). This should be preferred for high-linearity applications since the operational amplifier holds the voltage between its inputs ideally equal, turning the $R i$ resistors into linear voltage-to-current converters with performance limited only by the voltage dependence of polysilicon resistors (typically the smallest available on-chip). This also reduces possible nonlinear, signal-dependent effects on the DAC which have to be coupled to the same nodes.

In the topology chosen for this design the DAC is implemented as a set of 31 differential current sources controlled by a thermometer-coded, 31-bit word supplied by the loop quantizer (Fig. 7.2). By using equal unity DACs the signal-dependency of DAC switching is reduced compared to a binary-coded DAC [10]. The DACs are differential, hence the common-mode control loop at the opamp output is not loaded by the DAC switching because no common-mode current is integrated. That is why the DACs are not designed as current-steering (a topology preferred for high-speed DACs [6]), but as a set of PMOS (Vddreferred, sourcing) and NMOS (Gnd-referred, sinking) current sources which can be alternately connected to the opamp inputs, depending on corresponding comparator decision bit $B$. The matching requirements for the 31 current sources will be discussed later in this Chapter, Section 7.7, and a dynamic calibration method will be explained.

The two currents, signal and feedback, are integrated on the $\mathrm{Ci}$ capacitors by the opamp. These capacitors have to be voltage-independent down to $100 \mathrm{~dB}$ for the entire output voltage range. The operational amplifier should

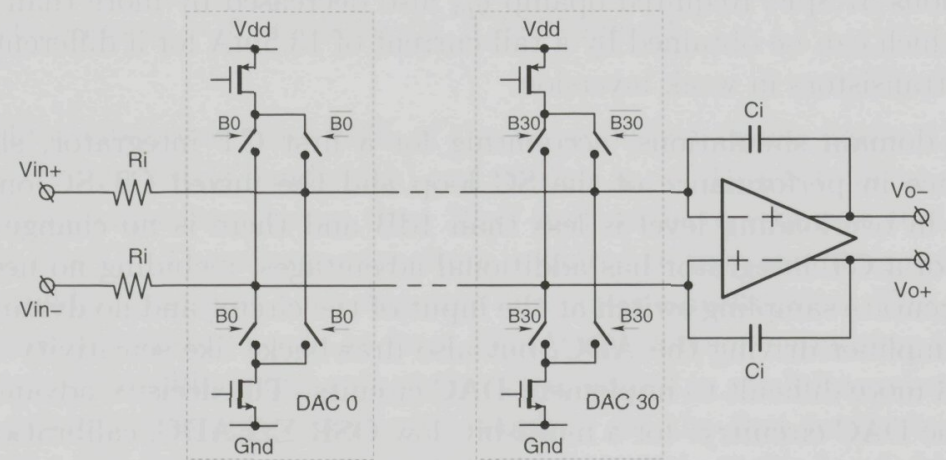

Figure 7.4: Topology of the CT integrator 
always operate in closed-loop, without slewing with a full-swing input and fullswing DAC current in phase. Its finite transconductance is the main source of nonlinearity by means of an opamp-input voltage which is dependent of the DAC output in a non-linear way, therefore modulating the integrated signal current. Depending on the operating region of the input-stage MOS transistors, there is a minimal opamp transconductance which guarantees the required linearity (see Chapter 4).

\section{Circuit Parameters}

As explained in sub-Section 7.2.3, the noise budget for the first integrator is $83 \%$ of the total noise budget. Based on the equations developed in Chapt 4 , the integration resistors and the opamp transconductance are calculated inside the CAD software as

$$
\begin{aligned}
& R_{i}=1.25 K \Omega \\
& g_{m}=135 \mathrm{mS}
\end{aligned}
$$

The $R i$ resistors have to be linear to $-100 \mathrm{~dB}$ over the entire input range, therefore polysilicon resistors have to be used on-chip, with widths larger than the minimal width to reduce their voltage dependence. The $g_{m}$ is calculated considering the opamp input transistors operated in weak inversion, which is almost always the case in low-voltage designs.

The integration capacitors $C i$ also have to show 16 bits linearity when the output of the first integrator swing close to the supply rails. Their size results form the first integrator's coefficient, the integration resistor and the clock period (the input signal is integrated for the entire clock period)

$$
C_{i}=\frac{T_{C L K}}{R_{i} a_{1}}=22.2 p F
$$

This value is slightly larger than the one reported by the CAD software and is caused by the choice of a smaller integration resistor, which should offer an extra margin for the in-band noise power.

The DACs are intended to be used in a Return-to-Zero configuration (RZ switches are not shown in Fig. 7.4 for the sake of schematic clarity) which means the differential current supplied during half clock period by each of them is

$$
I_{D A C}=\frac{1}{31} \frac{C_{i} a_{1} f_{1}}{T_{C L K}}=103 \mu A
$$

Because the conversion gain is $0 \mathrm{~dB}$, the differential input signal has a peak amplitude of $1.35 \mathrm{~V}(-2.5 \mathrm{~dB}$ compared to the reference value of $1.8 \mathrm{~V})$. This means a rail-to-rail input capability since only 2 saturation voltages are not covered near the supply rails. To take full advantage of the rail-to-rail capability, the common-mode voltage of the input signal has to be placed in the middle of the $1.8 \mathrm{~V}$ rail-to-rail voltage and the operational amplifier in the first integrator has to contain the value of $0.9 \mathrm{~V}$ in its common-mode input range. 


\section{Broadband, High Dynamic Range Sigma-Delta ADC}

\subsubsection{Operational Amplifier}

\section{Gain-Linearity Trade-off in Low-Voltage Design}

The only requirement deducted so far for the operational amplifier is to show an equivalent transconductance of $135 \mathrm{mS}$. This value guarantees that the DACinduced residual signal at the input of the opamp does not distort the integrated signal current more than $-100 \mathrm{~dB}$. There is however another source of nonlinearity which becomes important in low voltage design, namely the variation of the voltage gain with the output voltage. This is caused by the finite drain-source impedance of the output transistors, which typically is decreasing when process feature decreases. For the operational amplifier design this means it costs both more current and output voltage room to attain a given voltage gain under a gain linearity constraint with a single-stage amplifier when a digitally-advanced CMOS process is used.

The output range available with a single-stage opamp drastically decreases in $1.8 \mathrm{~V}$ designs compared to $3.3 \mathrm{~V}$ and $5 \mathrm{~V}$ equivalents. Since in $\Sigma \Delta \mathrm{ADC}$ design a lower output range means more-than-proportionally larger integration capacitor area, the most convenient solution is to use the 2-stage opamp shown in Fig. 7.5. The 2-stage class-A opamp offers a large DC gain without the need of cascodes in the second stage, therefore without any penalty on output range. Another advantage is a reduced input capacitance because the input transistors M1 and M2 are operated at lower current than the load-driving transistors M5 and M6. This reduces the capacitance driven by the opamp itself and by the driver in front of the ADC.

The tail current source of the input stage is cascoded to further improve common-mode rejection of the PMOS differential pair M1 and M2. The current sources $M 3$ and $M 4$ acting as active loads in the first stage are also cascoded

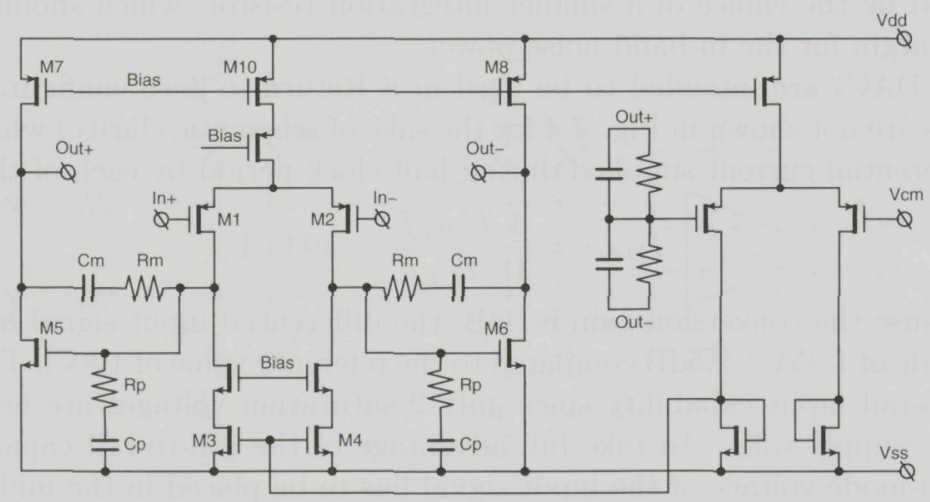

Figure 7.5: Class-A differential operational amplifier used in the first integrator 
so the output impedance of the first stage is only limited by the active transistors M1 and M2. Cascoding is not an option for these two devices because it introduces extra delay in the signal path, therefore reducing the phase margin. But because PMOS devices offer large output impedance than NMOS devices this is not a major drawback.

The second stage has rail-to-rail capability because no cascoding is done on either NMOS active transistors or PMOS current sources. The output impedance of the second stage is deliberately held low to reduce its voltagedependence. From the $60 \mathrm{~dB}$ DC voltage gain of the entire opamp, only $25 \mathrm{~dB}$ are supplied by the output stage. The gain of each half of the amplifier is still non-linearly dependent of the output voltage for the entire output range of $1.4 \mathrm{Vpp}$, as shown in Fig. 7.6. The curve is not symmetrical relative to zero volts ( Vout $=$ Vout $-=V c m$ ) so for the differential amplifier a net gain variation is present. Because the two halves of the curve are produced by variation of output impedance in the NMOS (M5, M6) and PMOS (M7, M8) transistors respectively, it is not possible to obtain a more balanced curve so its absolute variation is minimized by design.

The quiescent current in the input stage is $4 \mathrm{~mA}$, yielding a transconductance $g_{m 1}=31 \mathrm{mS}$. This transconductance drives the large gate-source capacitance of the output stage transistors (in excess of $2 \mathrm{pF}$ for transistors sized

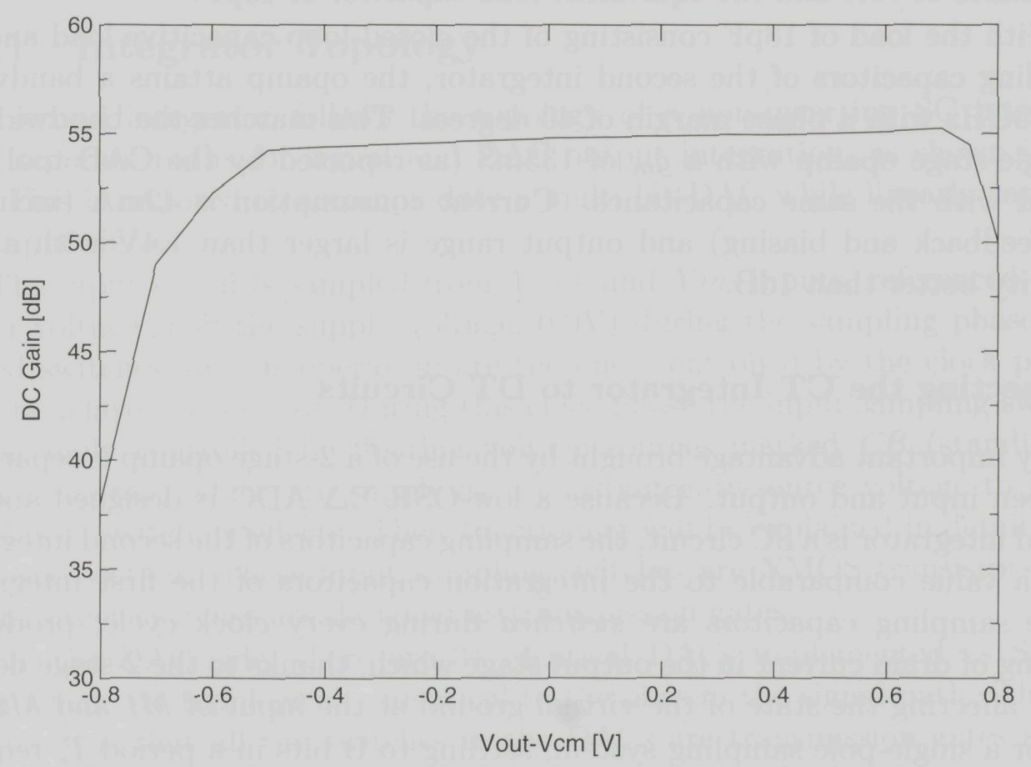

Figure 7.6: Variation of differential DC gain of the first amplifier with output voltage amplitude 
$W / L=4700 \mu \mathrm{m} / 0.18 \mu \mathrm{m}$ ) which are biased at $18 \mathrm{~mA}$ (each side) to attain a transconductance $g_{m 6}=320 \mathrm{mS}$. The output impedance of the wide NMOS output transistors is less than $70 \Omega$, which has two advantages: a low dependence of overall voltage gain to output voltage, and low inverting voltage gain for the gate-drain capacitance undergoing a Miller effect.

The latter fact is an advantage in the present design, since RC zeros are implemented in the compensation network to increase phase margin. Half of the compensation network is a Miller capacitor $\mathrm{Cm}$ in series with a resistor $\mathrm{Rm}$ sized to cancel the phase shift introduced by the positive zero at [7]

$$
Z_{m}=\frac{g_{m 6}}{2 \pi C_{m}}
$$

The resistor is actually sized to be larger than $1 / g_{m 6}$ so the negative zero shows at $600 \mathrm{MHz}$, one octave lower than the unity-gain bandwidth of $1.15 \mathrm{GHz}$ and 30 times lower than the actual positive zero frequency. The zero pre-warps the phase before the UGB frequency at the cost of a much more abrupt roll-off around the UGB.

The second half of the compensation network is built with $C p$ and $R p$ which introduce parallel compensation (convenient for its large bandwidth) and also a zero at $120 \mathrm{MHz}$ to cancel the effect of the output pole given by the output impedance of $70 \Omega$ and the equivalent load capacitor of $18 \mathrm{pF}$.

With the load of $18 \mathrm{pF}$ consisting of the closed-loop capacitive load and the sampling capacitors of the second integrator, the opamp attains a bandwidth of $1.15 \mathrm{GHz}$ with a phase margin of 40 degrees. This matches the bandwidth of a single-stage opamp with a $g_{m}$ of $135 \mathrm{mS}$ (as reported by the CAD tool) and loaded with the same capacitance. Current consumption is $42 \mathrm{~mA}$ (including $\mathrm{CM}$ feedback and biasing) and output range is larger than $1.4 \mathrm{~V}$ with a gain linearity better than $1 \mathrm{~dB}$.

\section{Connecting the CT Integrator to DT Circuits}

A very important advantage brought by the use of a 2 -stage opamp is separation between input and output. Because a low-OSR $\Sigma \Delta \mathrm{ADC}$ is designed and the second integrator is a SC circuit, the sampling capacitors of the second integrator have a value comparable to the integration capacitors of the first integrator. These sampling capacitors are switched during every clock cycle, producing peaking of drain current in the output stage which, thanks to the 2 -stage design, is not affecting the state of the virtual ground at the input of M1 and M2.

For a single-pole sampling system, settling to $\mathrm{B}$ bits in a period $T_{s}$ requires a system bandwidth

$$
U G B=\frac{B \ln (2)}{T_{s}}
$$


Settling requirement for the second integrator is 14 bits, as shown in Section 7.2 .3 with the CAD software output. Considering that sampling lasts only half of a clock period (i.e. $7.8 \mathrm{~ns}$ ), a bandwidth of $1.24 \mathrm{GHz}$ is required for the sampling system formed by the opamp in the first integrator and the sampling capacitors of the second integrator. Since the sampling capacitors of the second integrator are approximately one-half of the total capacitive load of the first integrator's opamp, the actual phase margin of the sampling system is larger than the 40 degrees mentioned above for the completely loaded opamp. The other half of the capacitive load is not switching and it actually helps during switching by reducing the high-frequency impedance at the output of the first integrator.

During initial design phase, the quiescent current of the output stage in the first integrator's opamp has been calculated to $14 \mathrm{~mA}$ on each side (Fig. 7.5) but has later been increased to $18 \mathrm{~mA}$ to correct the phase margin which would have otherwise affect settling performance for the sampling in the second integrator. As the next section will prove, the second integrator is as difficult to design a circuit as the first integrator, due to a combination of factors including large UGB in a low-voltage technology, low output impedance (hence low voltage gain) and high gate capacitance for the MOS transistors.

\subsection{Design of the 14-bit SC Integrator}

\subsubsection{Integrator Topology}

The second integrator follows the topology of a non-inverting SC integrator with separate paths for signal and DAC output integration, as shown in Fig. 7.7. This is required to accommodate a multi-bit DAC while linearly sampling the input signal.

The input signal is sampled from Vint and Vin-inputs, referenced to the $V c m i$ voltage (half the supply voltage, $0.9 \mathrm{~V}$ ) during the sampling phase. The closed switches for this operation are the ones controlled by the clock phase $s$ and its delayed version $s d$. During this clock phase the input sampling switches are actually controlled by floating voltage sources marked $C B$ (standing for clock bootstrap) which are supplying a constant gate-source voltage to reduce non-linear switching effects. Their functioning will be explained in detail in the next sub-section. These input sampling switches are NMOS transistors while all the other switches are designed as transmission gates.

On the DAC side, there are 31 identical DACs implemented as SC sections which are topologically identical to the ones in the signal path. The only difference is that all the switches in the DACs are transmission gates and no clock boosting is required because charge-transfer linearity is not an issue. The sampled voltage for each DAC is supplied by four switches which connect one $C f b$ capacitor to Vref and the other to Gnd according to each comparator's 


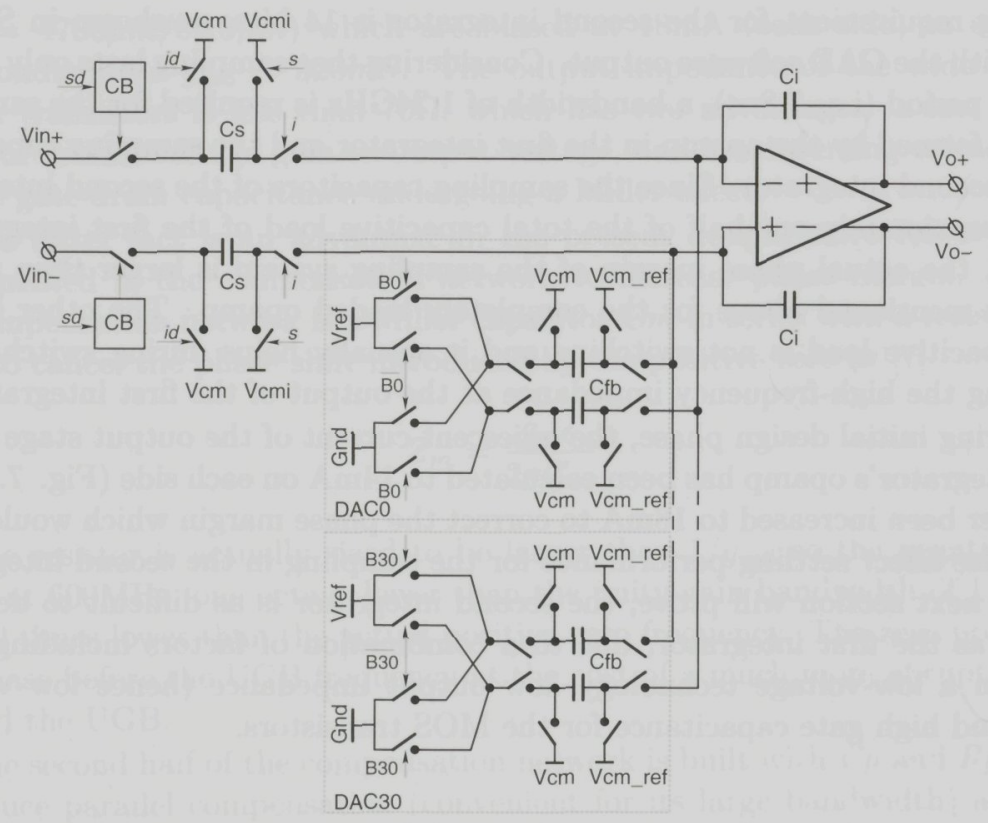

Figure 7.7: Complete topology of SC second integrator

decision. Both sampled voltages are referenced to Vcm_ref which is half Vref (again $0.9 \mathrm{~V}$ for $V r e f=1.8 \mathrm{~V}$ ). In this way, each $C f b$ capacitor is charged with $+/$ Vref $/ 2$, keeping the common-mode voltage at the input of the opamp to $0.9 \mathrm{~V}$. Floating feedback capacitors are not used here because they have one major asymmetry, the parasitic capacitor to substrate. A balanced configuration is preferred to reduce any signal-dependent switching effects.

All the capacitors are metal-metal capacitors to keep their linearity better than the required 14 bits. The parasitic capacitance for the integration capacitors is quite a large fraction of their nominal value and it adds a major capacitive load to the total load of the opamp. The parasitic capacitances associated with the switched capacitors are connected to the minimal impedance nodes, namely the signal inputs and Vref and Gnd.

\subsubsection{Linearized Sampling Switch}

The input sampling switches (on the signal path, Fig. 7.7) have to offer a low, signal-independent on-resistance during the sampling phase. The simplest option to implement these switches, with single MOS transistors, does not work because for one half of the input voltage range (the higher one for NMOS, 
lower one for PMOS) the switch stays off even if the clock voltage reaches the supply rail. Two other options span from here: one NMOS switch with doubleVdd clock signal or a transmission gate with a PMOS and an NMOS device in parallel. The clock-boosted option requires the use of a thick-oxide transistor, which is not convenient because the on-resistance for these devices is higher than for high-performance, thin-oxide devices. Also, both the clock-boosted and the transmission-gate options inject non-linear, signal-dependent charge in the signal path (see Chapter 3). The signal dependency of this charge, due to the fixed gate clock voltage, distorts the sampled signal and reduces the conversion linearity when the switches are used in a $\Sigma \Delta \mathrm{ADC}$ integrator.

A method that injects a fixed, signal-independent charge in the circuit is used in this design. The principle is explained in [8] and it consists in using a floating clock signal, setting the gate of an NMOS switch to a fixed voltage above its source in the $O N$ state. This means the $O N$ clock voltage is always constant, therefore the injected charge is signal-independent. The schematic of the clock bootstrap is shown in Fig. 7.8. When the CLK signal is low, transistors $M 11$ and $M 10$ connect the gate $G$ of the switch to Vss, keeping the switch in off-state (high impedance). During this time the battery capacitor C3 is charged to the supply voltage, the voltage doubler C1, C2, M1 and M2 supplying a $2 V d d$ switching voltage to the gate of $M 3$. When $C L K$ is high $(1.8 \mathrm{~V}), M 8$ and $M 9$ are switched on, connecting battery capacitor between the gate and the source of the switch.

Due to its floating operation, the bootstrapped clock only stresses the gate oxide of the switch for a short time (under 1ns) until a channel is formed, the voltage across the oxide dropping to the technology-tolerated $V d d$ after that.

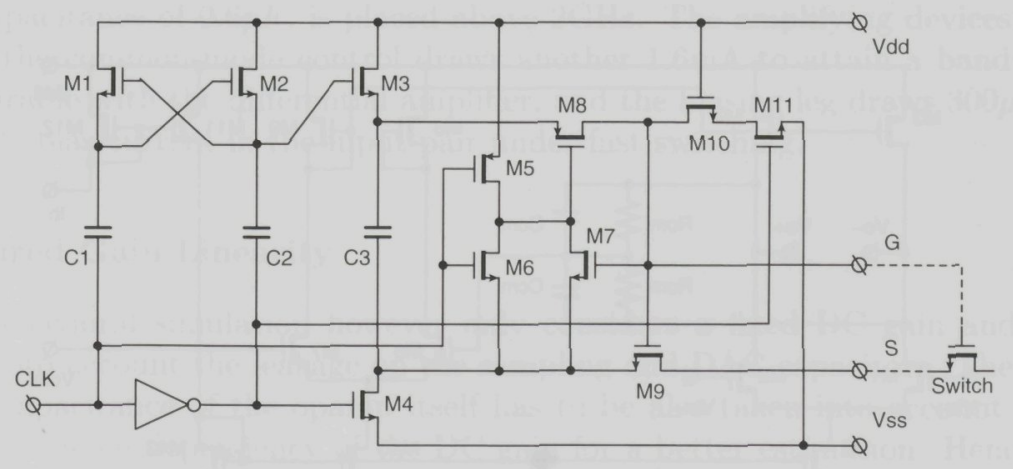

Figure 7.8: Clock boosting circuit 


\subsubsection{Capacitor Sizing}

Based on the CAD software output, the integration capacitors are $5.5 \mathrm{pF}$, sampling capacitors are $8.8 \mathrm{pF}$ and the total capacitance of the DAC is $7.7 \mathrm{pF}$. For 31 unitary DACs, this yields a $250 \mathrm{fF}$ capacitor per unit DAC, which is a value large enough to guarantee a $1 \%$ matching from unit-to-unit DAC. System level simulations show that, due to the low OSR used, such mismatch would cause a distortion in the order of $-80 \mathrm{~dB}$ even if it occurs in the second integrator's DAC. Therefore, a DWA circuit is employed between quantizer and second DAC to spread the mismatch across the sampled bandwidth (DC to $f_{s} / 2$ ), therefore reducing its in-band power and especially its peak spectral component.

\subsubsection{Operational Amplifier}

The operational amplifier for the second integrator has a single-stage topology. Its schematic is shown in Fig. 7.9. A very simple opamp topology has been chosen because, as opposed to cascaded $\Sigma \Delta$ ADCs, the single-loop approach is not especially sensitive to low opamp gain. Cascodes are not used for the $g_{m}$ pair $M 1-M 2$ because they reduce phase margin by introducing an additional pole in opamp's characteristic and additional capacitive load for the input $g_{m}$ pair. The cascodes also reduce the usable output voltage range.

The input devices are NMOS transistors because the input common-mode level allows one Vgs (either PMOS or NMOS) and one (large) V sat to either supply rails, and common-mode rejection is not a very restrictive issue in the second integrator. As a matter of fact, the main common-mode rejection decrease does not come from the back-gate transconductance effect [7], but from the reduced impedance of the tail current source, M5. Cascoding of this current

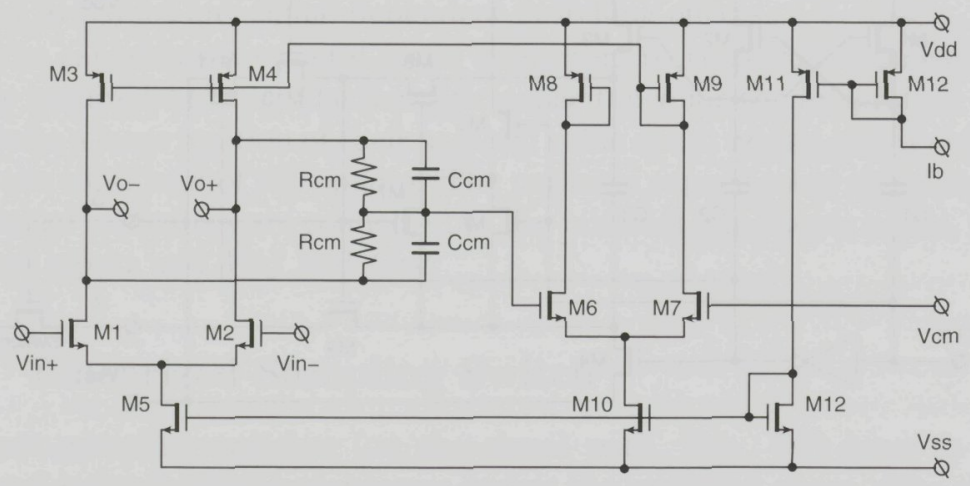

Figure 7.9: One-stage high-bandwidth opamp for SC second integrator 
source, with an available total drain-source voltage of $0.3 \mathrm{~V}$, does not significantly increase its impedance because not enough voltage is available to obtain a significant voltage gain in the cascode transistor.

The common-mode feedback loop is implemented as a CT circuit to minimize the dynamic capacitive load of the amplifier. The danger of instability of this loop is eliminated by using the MOS capacitors $\mathrm{Ccm}$ in parallel with the readout resistors, $R \mathrm{~cm}$. Power consumption in the biasing diode M12 is significant, in order to maintain a stable tail current for the fast-switching input pair.

\section{Required DC Gain and Bandwidth}

Behavioral simulations performed during design inside the CAD tool indicate that an opamp DC gain of only $24 \mathrm{~dB}$ is sufficient to guarantee the leakage in the SC integrators does not reduce DR. This gain is easily obtained with a one-stage amplifier, even without cascoding the MOS input pair. The amplifier has therefore been designed for a transconductance $g_{m}$ of $62 \mathrm{mS}$, lower than the one found by the CAD tool. This difference is caused by the fact that, in the simple switching model used to calculate the required $g_{m}$, the worst case in terms of input voltage is considered, namely a peak input voltage equal to $V d d$. This is not the case in a $\Sigma \Delta \mathrm{ADC}$, where the input signal in each integrator has a certain distribution that contains only a small percentage of peak input voltages. The simple $g_{m}$ calculation is still good for design comparisons but small corrections have to be made in the final circuit.

The equivalent open-loop capacitive load of the opamp is $25 \mathrm{pF}$, including DAC capacitors, sampling capacitors and parasitic capacitors. With this load a bandwidth of $370 \mathrm{MHz}$ is attained with 89 degrees phase margin. The second pole, caused by the switch on-resistance of around $100 \Omega$ and the opamp input capacitance of $0.6 p F$, is placed above $2 \mathrm{GHz}$. The amplifying devices draw $8 \mathrm{~mA}$, the common-mode control draws another $1.6 \mathrm{~mA}$ to attain a bandwidth comparable with the differential amplifier, and the biasing leg draws $300 \mu \mathrm{A}$ for a stable bias current in the input pair under fast switching.

\section{Required Gain Linearity}

The behavioral simulation however only considers a fixed DC gain and only takes into account the leakage on the sampling and DAC capacitors. The large input capacitance of the opamp itself has to be also taken into account along with the signal dependency of the DC gain for a better estimation. Hence, for a given clock index $n$, the output voltage $V_{\text {out }}^{+}$at the output of the amplifier is given by the previous input voltage $V_{i n}^{-}$, the sampling, integrating and DAC capacitors $C_{s}, C_{i}$ and $C_{f b}$ respectively, the previous output voltage $V_{\text {out }}^{-}$and the previous DAC voltage $V_{D A C}^{-}$, along with the opamp input capacitance $C_{p}$ 
and the signal- (and, therefore, time-) dependent DC gain, $A_{V}(n)$

$$
V_{\text {out }}^{+}=\frac{C_{s} V_{\text {in }}^{-}-C_{f} V_{D A C}^{-}+V_{\text {out }}^{-}\left(C_{i}+\frac{C_{p}}{A_{V}(n)}\right)}{C_{i}+\frac{C_{s}+C_{f b}+C_{p}}{A_{V}(n)}}
$$

Using this equation in behavioral simulations show that only a small gain non-linearity can be tolerated if the nominal voltage gain of the opamp is small. The designed differential gain for the opamp is $32 \mathrm{~dB}$, and it is a compromise between an $8 \mathrm{~dB}$ safety margin to the required value of $24 \mathrm{~dB}$ and the variation of drain-source impedance of the NMOS input pair across the output voltage range of $1.2 \mathrm{~V}$. The NMOS input devices have non-minimal lengths to increase their drain-source impedance, hence the voltage gain. This solution has been preferred over the cascoding because it produces a less variable output voltagedependent gain. Fig. 7.10 shows the variation of the voltage gain for one half of the opamp when its output voltage signal covers the entire output range. The two curves show this variation when the input NMOS pair is cascoded and when it is not. Even if in the latter case the gain is smaller, it is much less variable and its variations are more symmetrical around zero volts. The variation of the cascoded version is more than $25 \%$ at its peak while for the non-cacoded version the peak variation is $9 \%$ and is highly localized at the limits of the output range.

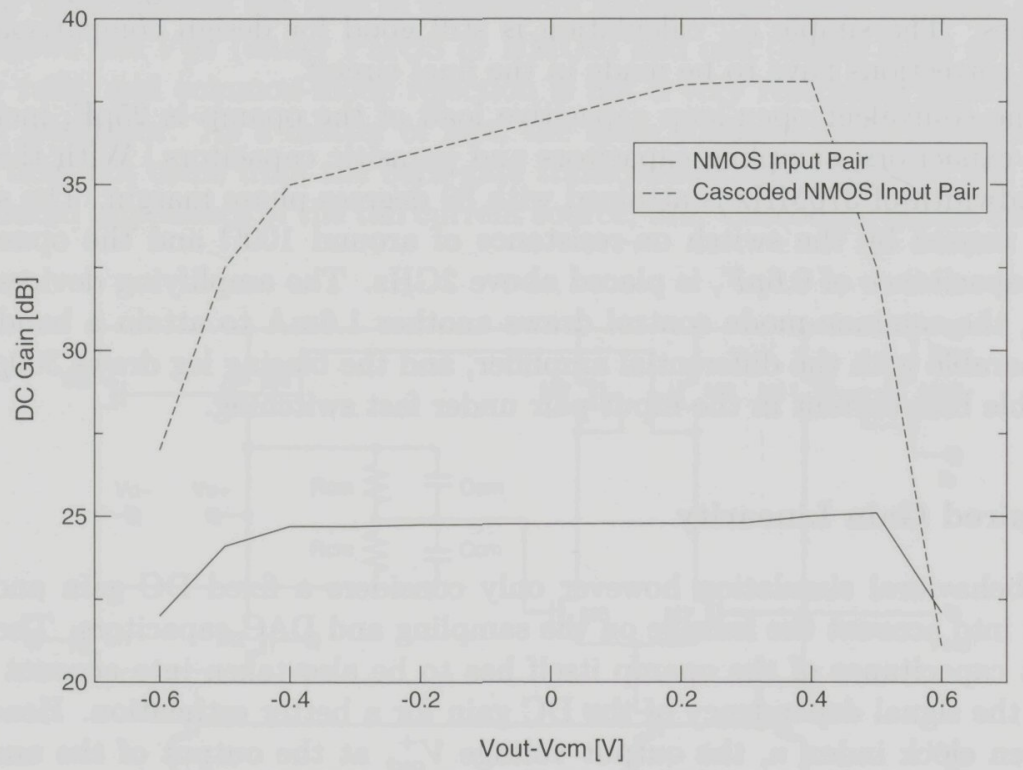

Figure 7.10: Variation of differential DC gain of the second amplifier with output voltage amplitude 


\subsection{Higher Order Integrators}

\subsubsection{Integrator Topology}

The third and the fourth integrator have topologies similar to the second integrator except for the DAC capacitors, as shown in Fig. 7.11. The floating-capacitor DAC is simplifying the design of the switches and no common-mode voltage has to be supplied. The common-mode voltage at the input of the opamp is only set by the signal integration path. Signal-dependent DAC switching shows negligible effects due to the large loop gain sustained by the first two integrators. The effect of signal-dependent charge injection in the sampling switches controlled by $s d$ is also negligible.

For the higher-order integrators the DAC capacitors are not noise-limited but mismatch-limited at circuit level. The CAD tool outputs a total DAC capacitance of $270 \mathrm{fF}$ for the third integrator, which is larger than the specified minimum capacitance of $200 \mathrm{fF}$, and only the fourth integrator has mismatchlimited capacitors. In the final design however, all DACs are actually split into 31 unity DACs, which for the third integrator equates

$$
C_{D A C}^{\text {unit }}=\frac{270 f F}{31}=8.7 f F
$$

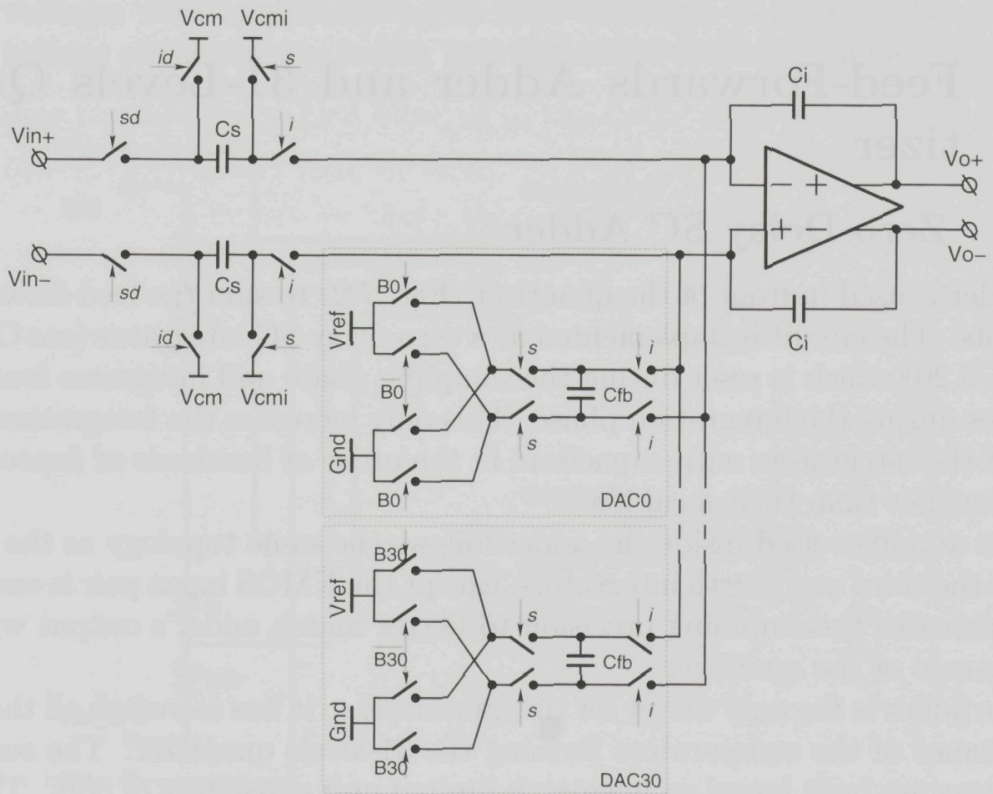

Figure 7.11: Topology of third and fourth SC integrators 
This value is too small to guarantee the required matching of $1 \%$ so all the capacitors in the third integrator are increased by a factor of 4.7 which yields convenient capacitor values. The same procedure is applied for the fourth integrator, with a multiplying factor of 6.3 .

\subsubsection{Capacitors Sizing and Opamps Performance}

The resulting capacitor sizes are listed in Table 7.6 along with the $g_{m}$ implemented by single-stage opamps. The opamps have the same topology as the one in the second integrator (single-stage, NMOS input) but the transistors are down-sized because the biasing currents are also smaller. The supply current for the third integrator is $3.2 \mathrm{~mA}$ and the one for the fourth integrator is $1.3 \mathrm{~mA}$. Unity-gain bandwidths for the opamps are $250 \mathrm{MHz}$ and $120 \mathrm{MHz}$ for equivalent open-loop capacitive loads of $7 \mathrm{pF}$ and $9 \mathrm{pF}$ respectively.

Table 7.6: Third and fourth integrator design parameters

\begin{tabular}{|l|c|c|c|c|}
\hline Integrator & $C_{s}$ & $C_{i}$ & $C_{D A C}^{\text {unit }}$ & $g_{m}$ \\
\hline Third & $2.25 \mathrm{pF}$ & $1.0 \mathrm{pF}$ & $40 \mathrm{fF}$ & $15 \mathrm{mS}$ \\
\hline Fourth & $2.4 \mathrm{pF}$ & $6.0 \mathrm{pF}$ & $38 \mathrm{fF}$ & $6 \mathrm{mS}$ \\
\hline
\end{tabular}

\subsection{Feed-Forwards Adder and 31-Levels Quan- tizer}

\subsubsection{Zero-Delay SC Adder}

An adder is used in front of the quantizer (Fig. 7.2) to sum the feed-forward coefficients. The circuit is implemented as a zero-delay SC integrator (see Chapter 3, Fig. 3.20) which is reset during the sampling phase and integrates four input voltages during the integration phase. This only increases the integration-phase load of the integrators with capacitors in the order of hundreds of femtofarads, much smaller than their local loads.

The amplifier used inside the adder follows the same topology as the amplifiers in the third and fourth integrators, except the NMOS input pair is cascoded. This increases the summing precision to better match adder's output with the input range of the quantizer.

The adder is the only driver for the quantizer, so it has to switch all the input capacitance of the comparators forming the 31-levels quantizer. The summing capacitors are built based on mismatch-limited unit capacitors of $50 \mathrm{fF}$. The first integrator's output is integrated on 6 capacitor units, and 10.5 units, 6.5 units and 8 units are used for the second, third and fourth integrator respectively. The 
summing capacitors are 18 units each to limit the adder's output voltage range to the input range of the quantizer, as will be explained in the next sub-section.

Equivalent capacitive load for the amplifier is $2 \mathrm{pF}$. The amplifier is supplied with $1.45 \mathrm{~mA}$ to attain a unity-gain bandwidth of $300 \mathrm{MHz}$. This large bandwidth guarantees that the dynamic comparators in the quantizer switch according to the the adder's output just before the adder is reset.

\subsubsection{Quantizer Topology}

Thirty-one comparators connected to a resistive ladder are used to build the 5bit quantizer, as shown in Fig. 7.12. The resistive ladder supplies the reference levels by dividing the reference voltage $V_{\text {ref }}=V($ Vref $)-V($ Ref_Gnd $)$ into 31 equal steps, with half step offset on either sides generated by the $R / 2$ resistors. The reference voltages are read differentially to reject common-mode switching noise. The differential input voltage $V_{i n+}-V_{i n-}$ is supplied to all comparators, while each comparator has a different reference voltage. Comparator number 30 (supplying decision bit B30) compares the input voltage with $\frac{30}{31} V_{\text {ref }}$, the next one with $\frac{28}{31} V_{\text {ref }}$ an so on until comparator number 16 has a reference voltage of zero volts $\left(V_{r-}\right.$ tied to $V_{r+}$ and both connected in the middle of the resistive ladder) and all following comparators have negative reference levels decreasing from zero to $-\frac{30}{31} V_{\text {ref }}$. For $V_{\text {ref }}=1.8 \mathrm{~V}$, the quantizer can process differential input voltages with amplitudes up to $1.8 \mathrm{~V}(3.6 \mathrm{Vpp})$ as long as the commonmode voltage of the resistor ladder and the one of the input signal match.

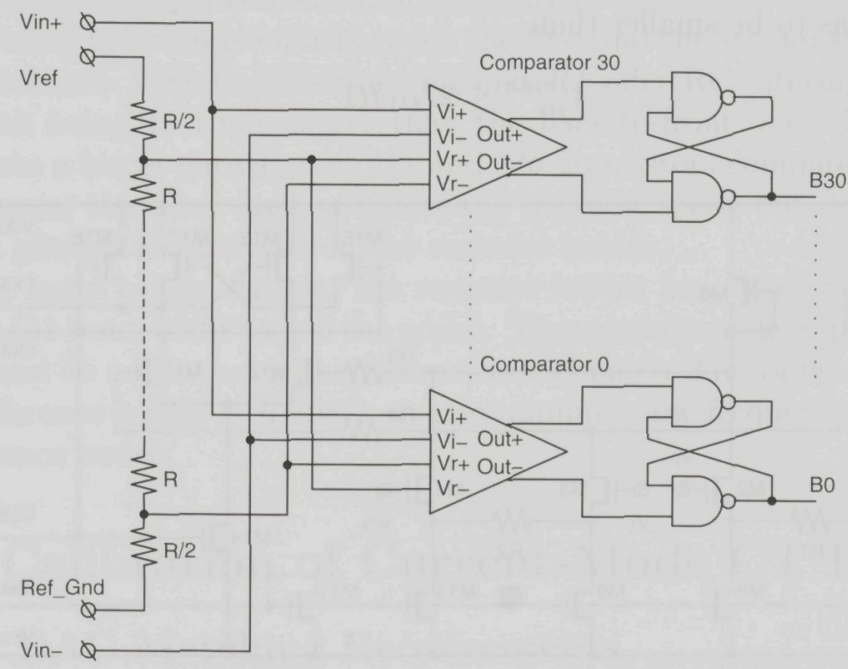

Figure 7.12: 31-levels quantizer 
Mismatches between different reference levels does not introduce significant distortion in the converted output, the loop gain from the converter input to the input in the quantizer being approximately $100 \mathrm{~dB}$ at the end of the signal bandwidth (see Chapter 2).

\section{Differential-Difference Dynamic Comparator}

For the low-voltage quantizer presented here, the differential-difference comparator shown in Fig. 7.13 has been designed. Two NMOS differential input stages, $M 1-M 2$ and $M 3-M_{4}$, are used to repeat the input and reference voltage on the floating resistors $R 1$ and $R 2$ respectively. The two resistors convert the voltages to differential currents which are subtracted in the diode-connected M5 and M6. The difference in gate-source voltages of M5 and M6 switches the latch formed by M16 and M17 by changing current balance in the second-stage differential amplifier M12-M13.

The biasing of the input stages is done with four separate current sources to maximize common-mode input range. The second stage is biased only on the $C L K=1$ (sampling phase) when the new decision is needed at the output. During $C L K=0$ phase the outputs Out + and Out-are tied to $V d d$ and the latch following the comparator (see Fig. 7.12) keeps the previous decision at the quantizer output.

\section{Differential Voltage Input Range}

Because the subtracting function has to be valid for the entire input range, the input signal has to be smaller than

$$
V_{\text {in }}^{\max } \leq 2 I_{D 1} R 1
$$

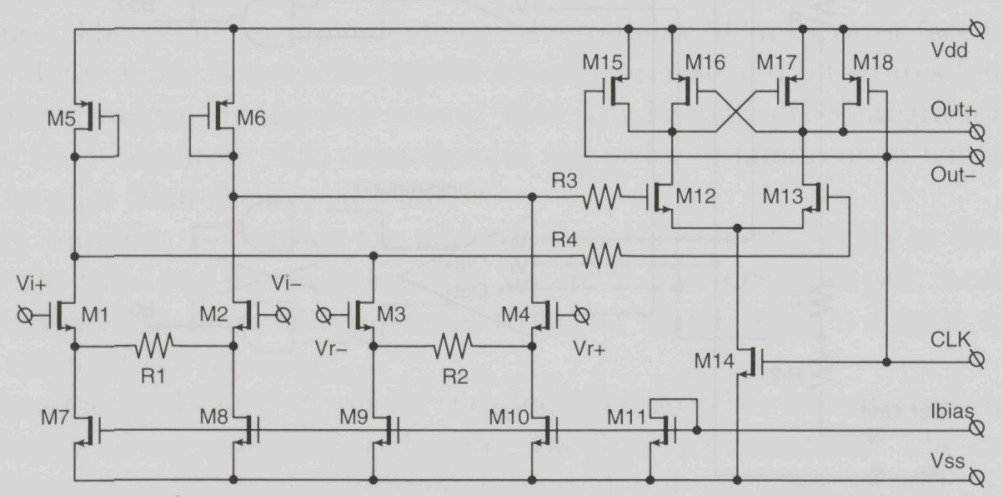

Figure 7.13: Differential-difference comparator 
assuming all input transistors are biased identically at $I_{D 1}$ drain current and the current sources biasing the input stages are saturated MOS transistors $\left(V_{D S} \geq\right.$ $V_{D S s a t}$ for M7-M10). The bias currents are designed for fast (around 1ns) switching of all gate capacitances connected to the drains of the input pairs, and they are $60 \mu \mathrm{A}$ for each input transistor. Resistors $R 1$ and $R 2$ are $31 \mathrm{k} \Omega$, kept at minimum to reduce both area and offset, and leaving a large margin between $V_{i n}^{\max }$ and $2 I_{D 1} R 1$. With these values, and taking into account the output voltage limits of the one-stage cascoded amplifier driving the quantizer (the opamp inside the SC adder), the entire adder plus quantizer ensemble is designed for a quantizer input amplitude of $0.8 \mathrm{~V}(1.6 \mathrm{Vpp}$ differential) when the input in the $\Sigma \Delta \mathrm{ADC}$ is close to the overloading level. That is why the summing capacitor in the adder (sub-section 7.6.1) is built with 18 unit capacitors instead of 8 . This drastic reduction of the input amplitude also guarantees that the comparator input stages are always biased by $M 7-M 10$ and, at full input swing, any input transistor has a enough drain current to keep the subtraction accurate.

\section{Comparator Kickback Minimization}

This two-stage design, besides providing the subtracting function in the first stage, also separates the fast-switching second stage from the resistive ladder supplying the reference voltage (see Fig. 7.12). The input stages are designed to work in a linear manner, with all input transistors being biased at non-zero drain current for all input voltage range. This minimizes comparator kickback which can introduce switching- (and therefore signal-) dependent reference levels, translating in A/D conversion non-linearity.

A filtering function is built between the first and the second stage to further reduce kickback. The two resistors $R 3$ and $R 4$ offer $\mathrm{RC}$ filtering, the forward bandwidth being 15 times larger than the back-to-front one. This difference comes from a larger gate area of M5 and M6 transistors compared to M12 and M13. Hence, the filter does not slow the decision speed but does reduce the kickback glitches as observed on the resistive ladder.

As an extra safety margin, the resistive ladder has small MOS capacitors attached (31 times 350fF) in all the nodes. These reduce node impedance for fast glitches and do not introduce significant area penalty. The value of the resistors in the reference ladder is $R=68 \Omega$, to also minimize low-frequency impedance in the reference nodes.

\subsection{Calibration of Current-Mode CT DAC}

\subsubsection{DAC Matching Requirements}

As opposed to single-bit $\Sigma \Delta$ ADC, the multibit designs suffer from nonlinear distortion induced by nonlinear DAC behavior. A single-bit DAC is inherently 
linear, since it only has two states. Any difference between the two states generates an offset and gain error but not harmonic distortion. Multi-bit DACs on the other hand have multiple states and their linearity is defined by the peak difference between any of these states and the line interpolated on the entire state set.

The DAC of the first integrator is the most critical one since its associated loop gain is unity, therefore its nonlinearity is not attenuated by the loop. The first DAC in this design is a 5-bit DAC built as 31 identical unity DACs, therefore its nonlinearity originates from unity DAC-to-DAC mismatch. Fig. 7.14 shows how DR and SNDR vary with unity DAC mismatch for the $\Sigma \Delta$ ADC designed here. The curves have been generated by behavioral simulations including normally-distributed DAC mismatch with peak values listed on the $\mathrm{X}$-axis. Mismatch values of $5 \%$ reduce the $\mathrm{DR}$ below $60 \mathrm{~dB}$ by means of harmonic distortion and modulation of out-of-band noise at lower frequencies. A mismatch better than $0.01 \%$ does not produce a significant increase in DR and SNDR values, because the power of harmonics induced by nonlinearity decrease below the in-band noise power.

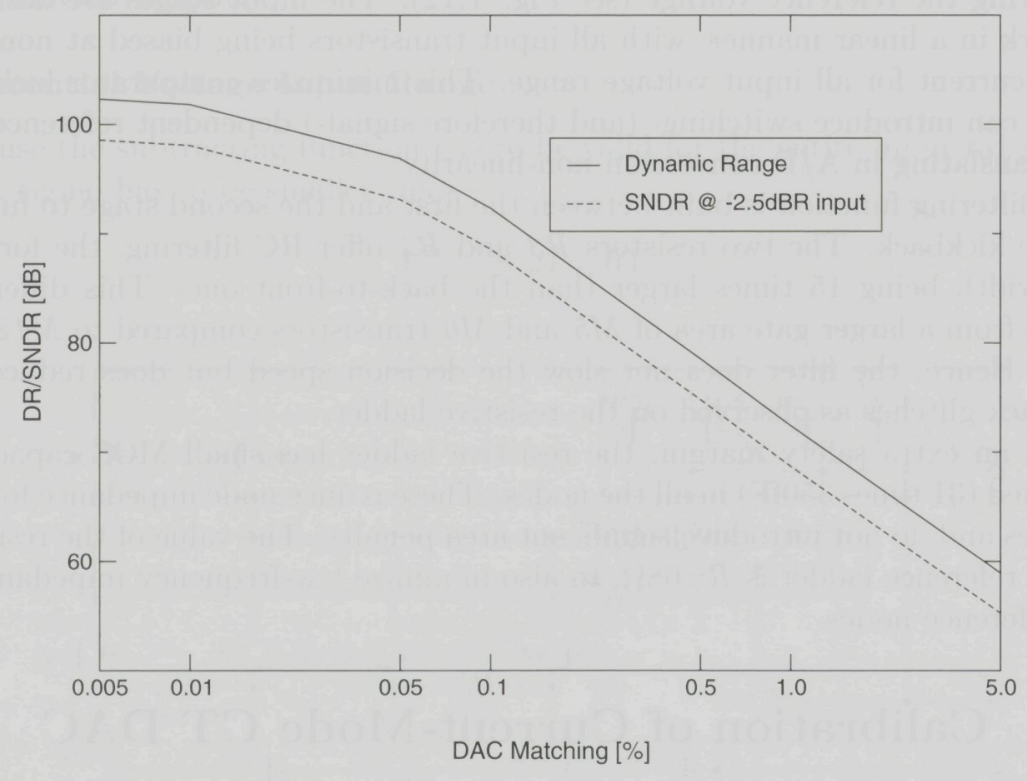

Figure 7.14: Decrease in DR and SNDR as a function of DAC matching 


\subsubsection{CT Current-Mode DAC}

As it has been shown in sub-Section 7.3.1, a set of 31 identical, pseudo-differential DACs are used in the first integrator. Each DAC is switched directly or crosscoupled to the opamp differential input (Fig. 7.4) according to the decision of the corresponding comparator in the quantizer. A more detailed schematic of each DAC is given in Fig. 7.15. The sink half is built with the NMOS transistor M1 while the source half is built with the PMOS transistor M2. Both current-source transistors are cascoded to increase their output impedance and therefore reduce the DAC current's sensitivity to the residual voltage variations at the opamp input (Fig. 7.4).

The switching scheme connects the two current sources to the opamp inputs. There are two PMOS switches controlled by the $P 1$ and $P 2$ switching phases and two NMOS switches controlled by $N 1$ and N2. By using two types of switches there is no need for double- $V d d$ clock signals. A return-to-zero switch controlled by $R Z$ connects the two current sources drain-to-drain for the half clock period when no DAC current is supplied to the integrator. The switching signals are generated to each DAC by a local clocks generator CLOCK which derives the P1, P2, RZ, N1 and N2 signals from the master clock signal and the bit decision of the corresponding comparator, $B$. The switching signals are generated as nonoverlapping digital clocks, as shown in Fig. 7.15 by the waveforms depicted in the lower-right corner. If the decision is $B=1$, on the integrating phase $C L K=0$ the $R Z$ is inactive $(R Z=0)$ while the NMOS source is connected to IDAC2 $(N 1=1)$ and the PMOS source is connected to IDAC1 $(P 1=0)$, decreasing first integrator's output (Fig. 7.4). The other two switches are in high-impedance mode, $N 2=0$ and $P 2=1$. During the second half of the clock period, $R Z$ is activated while all the other switches are in high-impedance mode.
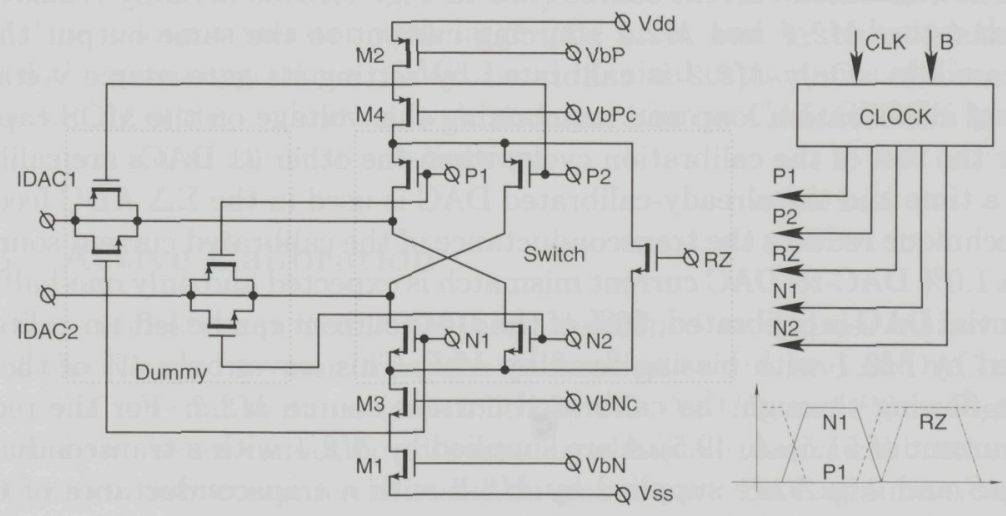

Figure 7.15: CT current-mode DAC 
At the IDAC outputs, two cross-coupled dummy switch pairs compensate for the charge injection introduced by the main current switches. The dummies are half the size of the current switches and they only compensate for the commonmode charge injection. This reduces the fast peak currents during switching, therefore reducing the dynamic load for the opamp in the first integrator.

As it has been explained in the previous sub-Section, the CT DACs have to be matched to $-80 \mathrm{~dB}$ in order to attain the target $-100 \mathrm{dBc}$ distortion. Such matching cannot be attained only with good layout techniques or large transistor gate area, so either a dynamic matching or a calibration procedure has to be applied. In this design the calibration is preferred for its less-spurious output [2] [10]. The target of this calibration is the difference between the currents sunk and sourced by each DAC (Fig. 7.15) and not the exact value of each of these currents thanks to the common-mode rejection of the opamp integrating the DAC currents. Therefore, only one calibration per DAC is required.

In this design the calibration procedure is a background calibration, with 32 DACs actually implemented in total but only 31 of them used at any given time for $\Sigma \Delta$ feedback. Meanwhile, the spare DAC is calibrated. Each DAC is calibrated once per calibration cycle. During each DAC calibration slot, the gate-source voltage of the target current source in that particular DAC is corrected in a linear manner by a calibration loop, and the corrected voltage is memorized on a capacitor until the next calibration cycle. It is more convenient to apply this calibration to the PMOS current source to take advantage of its reduced transconductance compared to the NMOS counterpart. Because a change of the memorized gate-source voltage is expected to take place between two calibration cycles, a reduced transconductance means this change has less effect on the value of the sourced current.

A detailed schematic of the calibration hardware in each DAC is given in Fig. 7.16. The calibrated current source (M2 in Fig. 7.15) is actually realized with two transistors M2_1 and M2_2 sourcing current to the same output through their cascodes. Only M2_2 is calibrated by setting its gate-source voltage by means of a calibration loop and memorizing this voltage on the MOS capacitor $\mathrm{Cm}$ for the rest of the calibration cycle, when the other 31 DACs are calibrated one at a time and the already-calibrated DAC is used in the $\Sigma \Delta$ ADC feedback. This technique reduces the transconductance of the calibrated current source [9]. Since a $1.0 \%$ DAC-to-DAC current mismatch is expected and only one-half of the differential DAC is calibrated, $96 \%$ of the DAC current can be left un-calibrated, supplied by M2_1 with biasing fixed by $V b P$. This leaves only $4 \%$ of the DAC current flowing through the calibrated current source M2_2. For the required DAC current of $51.5 \mu \mathrm{A}, 49.5 \mu \mathrm{A}$ are supplied by $M 2_{-} 1$ with a transconductance of $475 \mu \mathrm{S}$ and $2.0 \mu \mathrm{A}$ are supplied by $\mathrm{M}_{2} 2$ with a transconductance of $6.0 \mu \mathrm{S}$. Therefore, for a $-80 \mathrm{~dB}$ change in DAC current (which means $\Delta I_{D A C}=2.5 n A$ during one calibration cycle) the gate-source voltage memorized on $\mathrm{Cm}$ can vary 

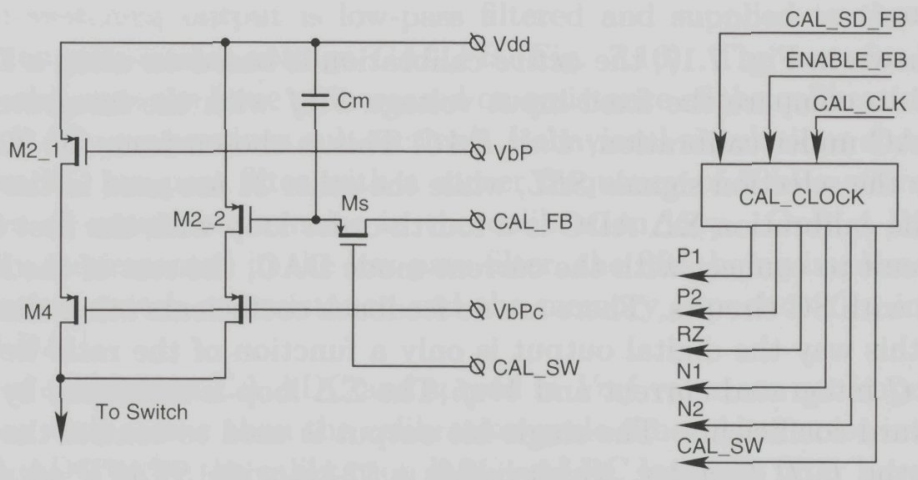

Figure 7.16: Detail of calibrated PMOS current mirror for CT current-mode $D A C$

with

$$
\Delta V_{g s}=\frac{\Delta I_{D A C}}{g_{\text {mcal }}}=416 \mu \mathrm{V}
$$

This voltage change has two different causes, the finite off-resistance of the calibration switch $M s$ and the capacitive coupling from the output of the DAC to the gate of the calibration PMOS, M2_2. The effect of Ms finite off-resistance can be reduced by increasing transistor's length, therefore attaining better separation from $C A L_{-} F B$ node to the gate of M2_2. This generates two negative effects, larger on-resistance and larger charge injection when $M s$ switches. It will be shown in the next section that both these drawbacks can be successfully corrected by means of calibration loop design. The effect of capacitive coupling from DAC output to the gate of M2_2 is reduced by designing the cascode of M2_2 as a long transistor with large gate area, and by reducing the ratio between the gate capacitance of M2_2 and nominal capacitance of $\mathrm{Cm}$.

The timing of the calibration cycle and operation of $C A L_{-} C L O C K$ generator will be explained in the next sub-Section.

\subsubsection{Active Calibration}

An active calibration principle has been developed to address mainly the effects of the charge injection from the calibration switch and of DAC-to-DAC mismatch of internal switches (controlled by N1, N2, P1 and P2 in Fig. 7.15). Both these two issues cannot be addressed by classical, passive calibration methods [9], which only compare the static output of each DAC against a reference value to correct it. With all these procedures the switches involved in selecting the calibrated DAC are completely different from the ones actually used in DAC 
operation.

As shown in Fig. 7.17, the active calibration is based on using a $\Sigma \Delta$ singlebit ADC to compare the fixed input voltage Vref with the integrated current of the DAC under calibration, Unit DAC. This is chosen from the 32 available DACs by the selection signals $S E L$, while the other 31 are used in the main $\Sigma \Delta$ ADC. The calibration $\Sigma \Delta \mathrm{ADC}$ is a fourth-order loop with the first integrator a CT circuit to connect with the current-mode DAC, the rest of the loop being designed with SC circuits. There are no feedback coefficients other than the first one. In this way the digital output is only a function of the ratio between the Unit DAC integrated current and Vref. The $\Sigma \Delta$ loop is stabilized by means of feed-forward coefficients. The single-bit output is used to control the up/down input of the $U / D$ Counter, which builds a 12-bit-wide STATE output as the integral of the ratio between the input voltage Vref and Unit DAC integrated current. For an ideal DAC current value the ratio is 4 (Vref is $-12 \mathrm{dBR}$ in terms of $\Sigma \triangle A D C$ design), therefore the STATE value is shifted left twice and compared to the current calibration slot state, CRT_STATE. The CRT_STATE value is generated by a counter counting upwards and reset at the beginning of the calibration slot, when a new Unit $D A C$ is selected. The two values are read by a 31-steps comparator (5-bit Comparator) which memorizes the last 30 values of CRT_STATE and compares them against the shifted STATE to generate a 5 -bit output proportional with the difference between the two inputs, $A-B$. This 5-bit signal is used to activate a binary-coded DAC, Calibration DAC,

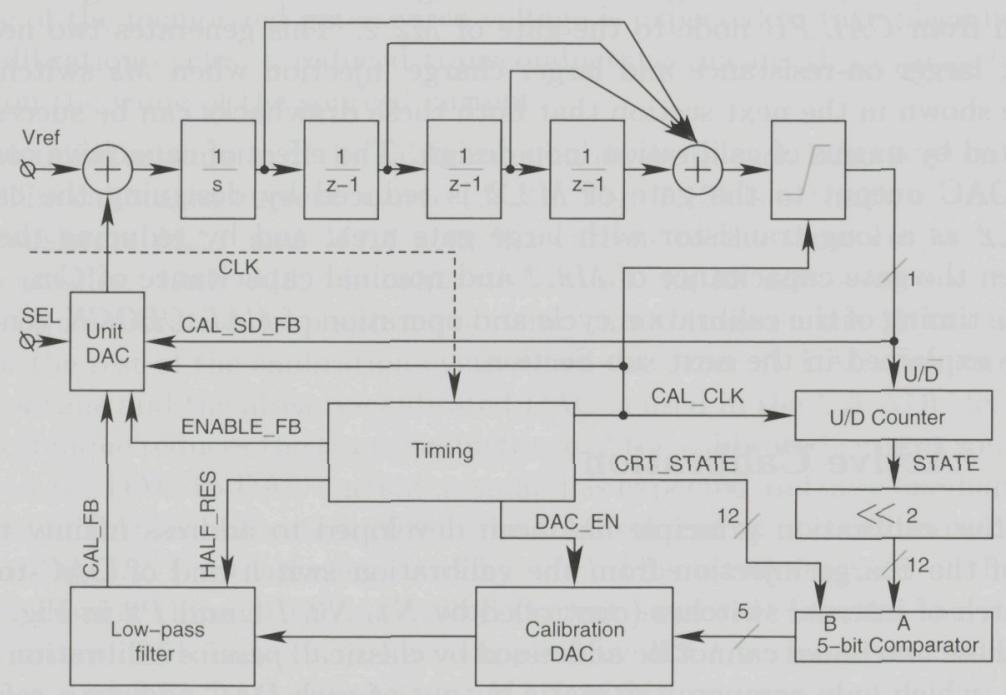

Figure 7.17: Calibration loop for the CT current-mode DAC 
whose fast-switching output is low-pass filtered and supplied to the DAC as the corrected gate-source voltage $C A L_{-} F B$ (Fig. 7.16). The use of a low-pass filter also addresses the issue of increased on-resistance of the calibration switch inside the DAC (see previous sub-section). Behavioral simulations showed that a 5 th order RC low-pass filter with a corner frequency of $60 \mathrm{kHz}$ offers enough attenuation of quantization noise in the calibration loop. Only $4 \mathrm{RC}$ stages are actually implemented in the low-pass filter, the fifth being implemented by the calibration switch on-resistance and the memory capacitor $\mathrm{Cm}$ inside the calibrated DAC.

Offset of calibration $\Sigma \Delta \mathrm{ADC}$ and spread in Vref value are not important as long as they drift slower than the calibration cycle time. Also, noise introduced by the $\Sigma \Delta \mathrm{ADC}$ or by the calibration DAC and RC low-pass filter have relaxed specifications because of the low bandwidth of the loop.

The timing of the entire calibration slot and the switching from one slot to the next (change of the Unit DAC under calibration) is controlled by a Timing block. This block supplies the calibration clock $C A L_{-} C L K$ which is the main clock $C L K$ divided by 8 . The calibration clock frequency of $8 \mathrm{MHz}$ reduces power consumption in the calibration $\Sigma \Delta \mathrm{ADC}$ and all additional digital circuits. When the slot begins, the Timing block activates the HALF_RES signal for one-third of the slot to double the corner frequency of the low-pass filter and therefore help the calibration loop to faster correct large changes required in the Unit DAC. Also, for $16 C A L_{-} C L K$ cycles the Calibration DAC is not activated, so the control loop is not closed until the 5-bit Comparator has half of its register bank filled with correct values.

To address the issue of charge injection by the calibration switch, as explained in the previous sub-Section, the calibration timing is controlled in such a way that the charge is injected inside the calibration loop. The most important calibration waveforms are plotted in Fig. 7.18. For half $C A L_{-} C L K$ period $\left(C A L_{-} C L K=1\right)$ all the DAC switches are inactive and the DAC under calibration is in return-to-zero mode while the gate-source voltage of M2_2 (Fig. 7.16) is connected to the calibration loop through the calibration switch $M s$ $\left(C A L_{-} S W=0\right)$. The DAC is actually used inside the calibration $\Sigma \Delta \mathrm{ADC}$ for only half of $C L K$ period (for the $64 \mathrm{MHz}$ clock this means $7.8 \mathrm{~ns}$ ) when $E N$ $A B L E_{-} F B$ is asserted by the Timing block. With this timing, charge injected in the memory capacitor by the calibration switch is accounted for during the comparison period ( $\left.E N A B L E_{-} F B=1\right)$ and therefore corrected by the calibration loop.

The evolution of normalized calibration error for one calibration slot is plotted in Fig. 7.19, for a DAC showing the peak mismatch of 3.5\%. The graph shows that, after a fast and steady decrease for the first 1000 clock cycles (when the low-pass filter has double bandwidth), the error starts to show a cyclic behavior caused by the tones in the $\Sigma \Delta \mathrm{ADC}$, the reduction of these tones being the main reason why a second-order $\Sigma \Delta \mathrm{ADC}$ cannot be used. With the fourth 
Broadband, High Dynamic Range Sigma-Delta ADC

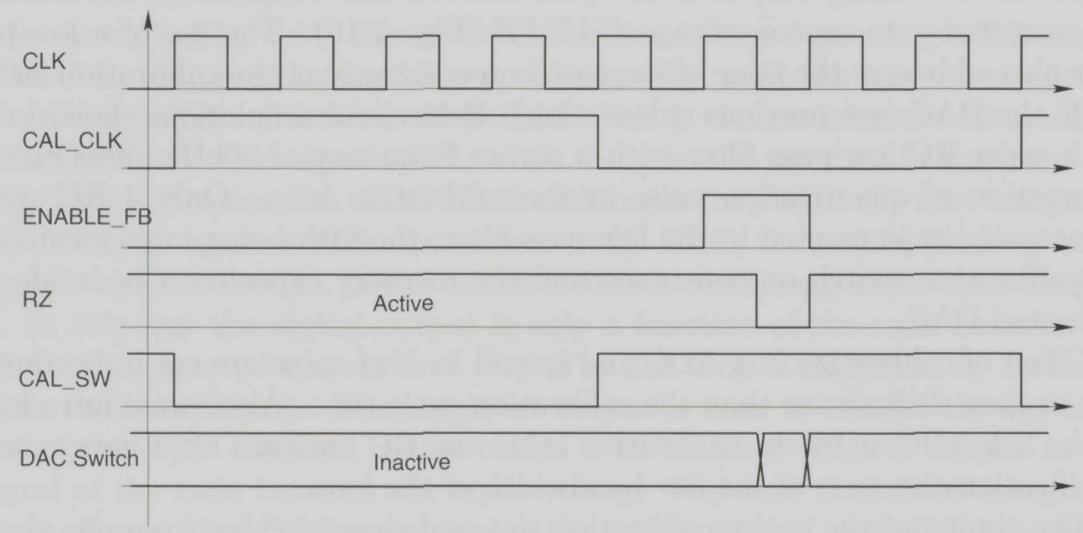

Figure 7.18: Calibration timing

order $\Sigma \Delta \mathrm{ADC}$ however, even the peak error is below the target $0.01 \%\left(10^{-4}\right)$ after 3000 clock cycles, so the entire calibration slot takes 3072 (a convenient

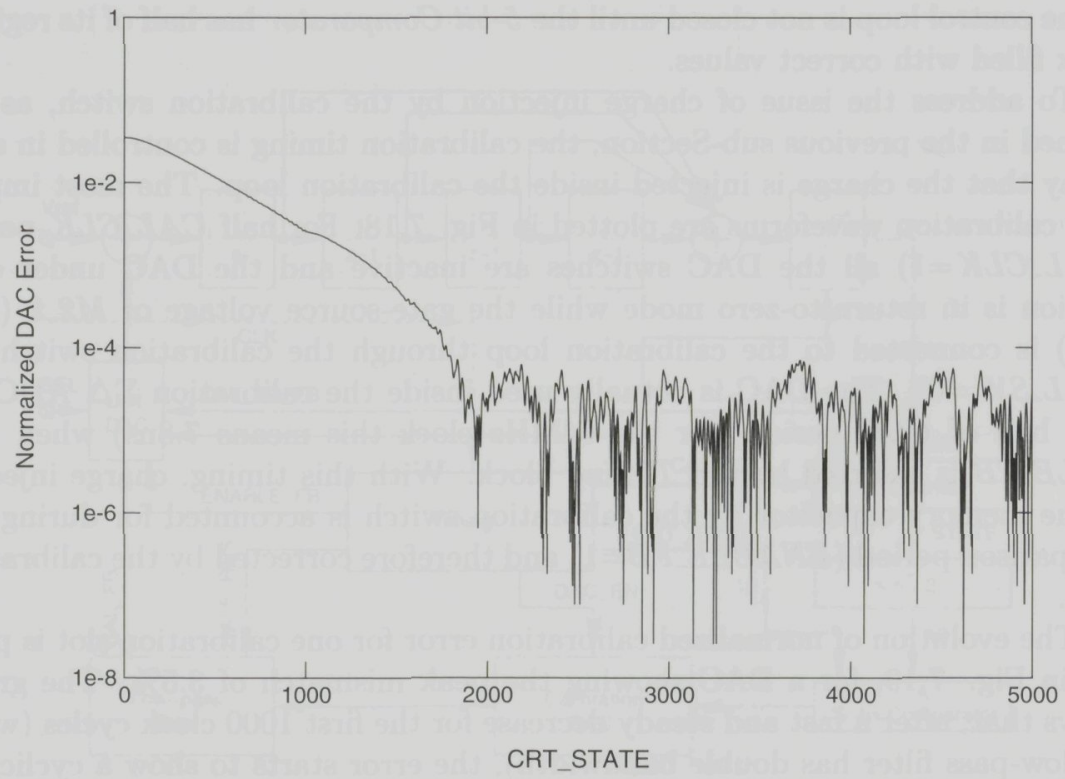

Figure 7.19: DAC calibration error as a function of slot time, CRT_STATE 188 Systematic Design of $\Sigma \Delta$ ADCs 
binary value, equals $2^{11}+2^{10}$ ) clock cycles. With an $8 \mathrm{MHz}$ calibration clock,

$$
T_{\text {slot }}=\frac{3072}{8 M H z}=384 \mu \mathrm{s}, T_{\text {cycle }}=32 T_{\text {slot }}=12.3 \mathrm{~ms}
$$

\subsubsection{Calibration DAC}

For a certain slot time, the amplitude of the calibration error is directly proportional with the number of bits used in the calibration DAC which supplies the signal for the low-pass filter. The design trade-off is between the amount of quantization noise put in the low-pass filter and the cutoff frequency of this filter, to reach a variation smaller than $0.01 \%$ at the filter output. A longer calibration slot means tougher requirements for the calibration transistor M2_2 (Fig. 7.16) and its associated calibration switch $M s$. Therefore, the number of bits in the calibration DAC has been set to 5 , which is increasing the complexity of this part of the calibration loop but shortens calibration time.

A large increase in complexity appears in the digital comparator supplying the calibration DAC's 5-bit inputs. As shown in Fig. 7.20, the comparator consists of 30 registers which contain the previous 30 values of CRT_STATE and 31 comparators which compare the current value of STATE (input $A$ ) with the current value of CRT_STATE (input $B$ ) and the registered 30 previous values, the result of the 31 comparators being a thermometer-coded 31-bit word which is binary-coded by a 31 to 5 encoder.

The 5-bit binary code is used to switch the calibration DAC designed with binary-divided current sources, as shown in Fig. 7.21. The DAC can be disabled,

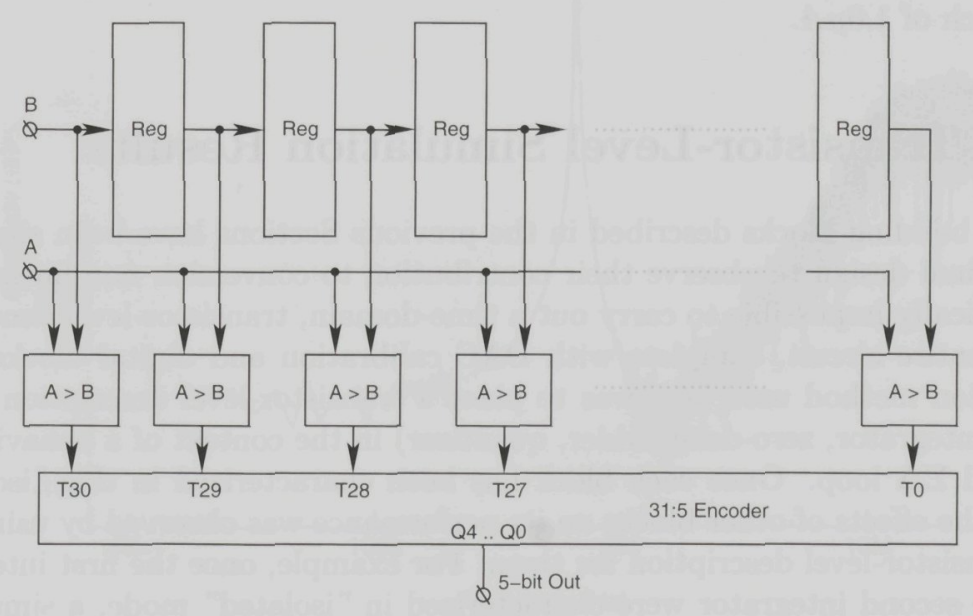

Figure 7.20: Digital 31-step comparator with binary-coded 5-bits output 


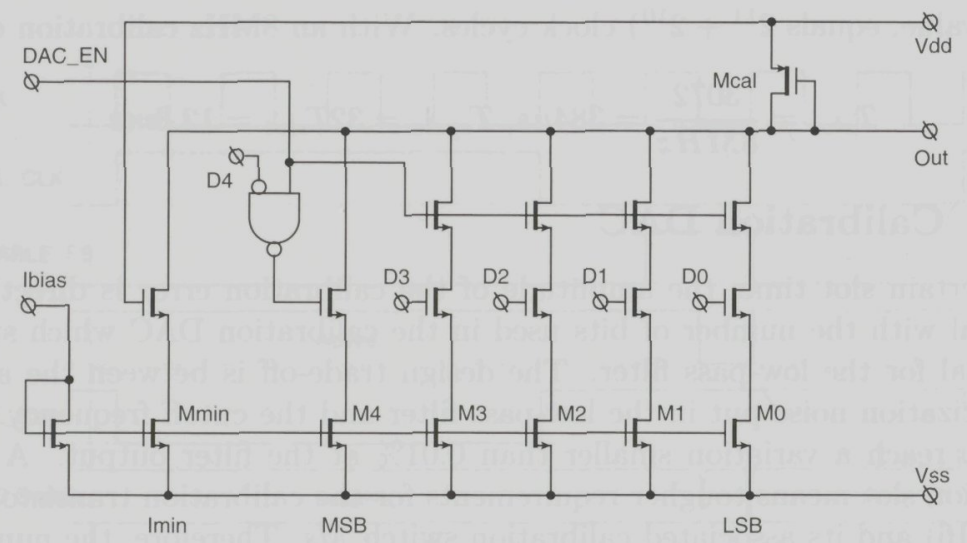

Figure 7.21: Binary-coded calibration DAC

leaving only a minimal current of $10 \mu \mathrm{A}$ plus the MSB current of another $10 \mu \mathrm{A}$ to bias the diode-connected $\mathrm{Mcal}$ which is 10 times the size of the calibration transistor in each DAC, M2_2 (Fig. 7.16). In disabled state the input word D4 to DO has no effect.

When enabled, the input word defines the state of the calibration DAC. The transistors $M 4$ to $M 0$ sink binary-divided currents from $\mathrm{Mcal}$, with a full-scale current of $19.4 \mu \mathrm{A}$. The biasing current range for $\mathrm{Mcal}$ is therefore $10 \mu \mathrm{A}$ to $30 \mu \mathrm{A}$, which translate into $1 \mu \mathrm{A}$ to $3 \mu \mathrm{A}$ for the calibration transistors M2_2 in each DAC. This range is sufficient to cover the expected $3 \sigma$ DAC-to-DAC mismatch of $1.0 \mu \mathrm{A}$.

\subsection{Transistor-Level Simulation Results}

All the building blocks described in the previous Sections have been simulated in the final design to observe their contribution to conversion non-linearity. It is practically impossible to carry out a time-domain, transistor-level simulation of the entire circuit, complete with DAC calibration and digital blocks. The simulation method used here was to place a transistor-level description of one block (integrator, zero-delay adder, quantizer) in the context of a behaviorallymodeled $\Sigma \Delta$ loop. Once each block has been characterized in this "isolated" mode, the effects of other blocks on its performance was observed by using partial transistor-level description for them. For example, once the first integrator and the second integrator were characterized in "isolated" mode, a simulation was run using full transistor-level model for the first integrator, loaded by a transistor-level model of the second-integrator's sampling capacitors with their 
switches; in the second integrator, the opamp was modeled as a functional block to speed up simulation without reducing its accuracy. The most complex simulation was carried out for the combination of the transistor-level model of the zero-delay adder and the transistor-level model of the quantizer.

The distortion introduced by the first integrator is usually the most important since it is not attenuated by the loop gain. In this design, not only the integration distortion introduced by the finite, non-linear $g_{m}$ of the opamp is important, but also the effect of the second integrator's sampling capacitors which have to be driven by the first opamp. These coupled effects have been simulated by considering linear (ideal) multibit DACs for the first and second integrators. The spectrum of the output of the $\Sigma \Delta$ ADC with a single-tone, $-3 \mathrm{dBR}$ input signal is shown in Fig. 7.22. The FFT bin is $6.6 \mathrm{kHz}$ (4800 bins from DC to $32 \mathrm{MHz}$ ). From this spectrum, estimated DR is $100 \mathrm{~dB}$, which is smaller than the expected quantization-noise-limited number. The extra noise is a common problem in relatively short, time-domain circuit simulations. Its source is the numerical noise, and the DR measurements on the chip are likely to be closer to the behavioral-level simulations than to the circuit-level ones. The spread of the input tone is caused by the window used to limit bin-to-bin leakage. A longer output trace (smaller bin size) was difficult to reach. As is, the result has been obtained after a 20 -hour simulation on a high-performance workstation. The most important result is the third harmonic distortion, at

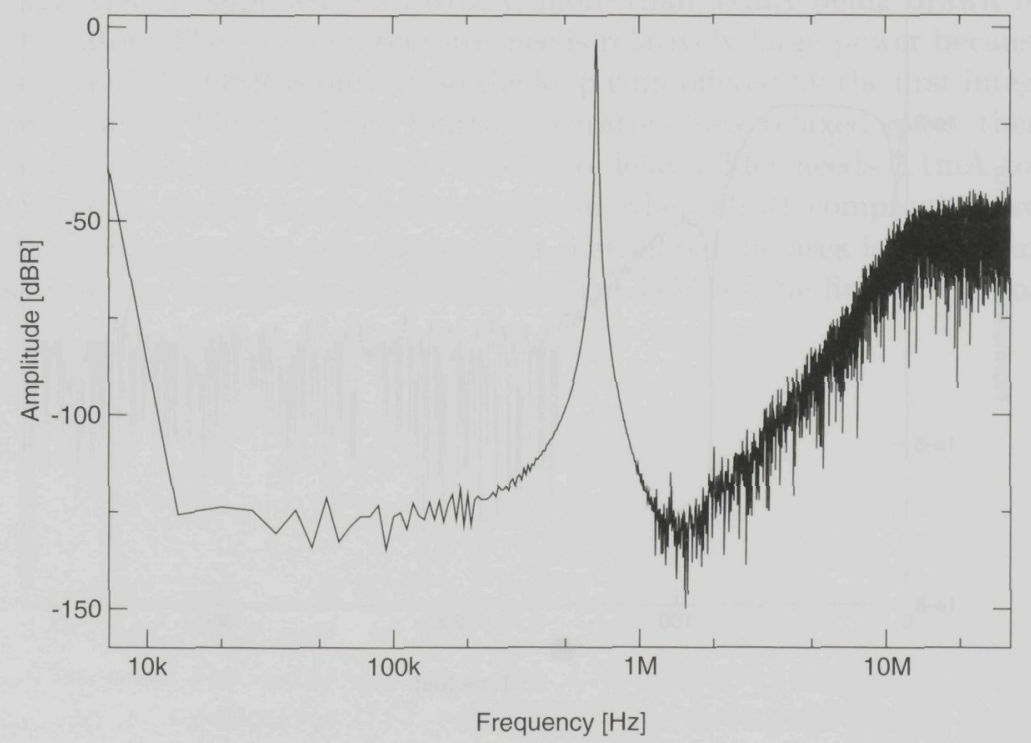

Figure 7.22: Spectrum of the digital output simulated at transistor-level 
$-103 \mathrm{~dB}$ compared to the main tone. It shows that the first integrator can attain the required performance while driving relatively large switched capacitors. The high-frequency part of the spectrum shows good noise shaping and a peak noise value matching the behavioral-level simulations.

For the first integrator, the linearity of the DAC has to be corrected by calibration. The efficiency of the active calibration explained in the previous Section has been analyzed by simulating at transistor level the critical analog blocks involved (the DAC under calibration, the calibration 5-bit DAC and the filters) along with behavioral-level descriptions of the digital blocks and of the calibration $\Sigma \Delta$ ADC. The evolution in time of the relative calibration error during one calibration slot $(384 \mu \mathrm{s})$ is shown in Fig. 7.23 . It can be seen that the error goes below the desired $10^{-4}(-80 \mathrm{~dB})$ after $300 \mu \mathrm{s}$ even if, at the beginning of the calibration slot, the unit DAC showed a mismatch in excess of $1 \%$. The flat region in error evolution at the beginning of the calibration slot shows the time required by the calibration $\Sigma \Delta \mathrm{ADC}$ and the entire filtering path to reach their steady-states. This only happens at power-up and represents the worstcase for the calibration process. The following calibration slots and cycles do not reset the calibration loop, so they are likely to reach the target error faster than in Fig. 7.23.

The simulated performance is summarized in Table 7.7. An area estimate,

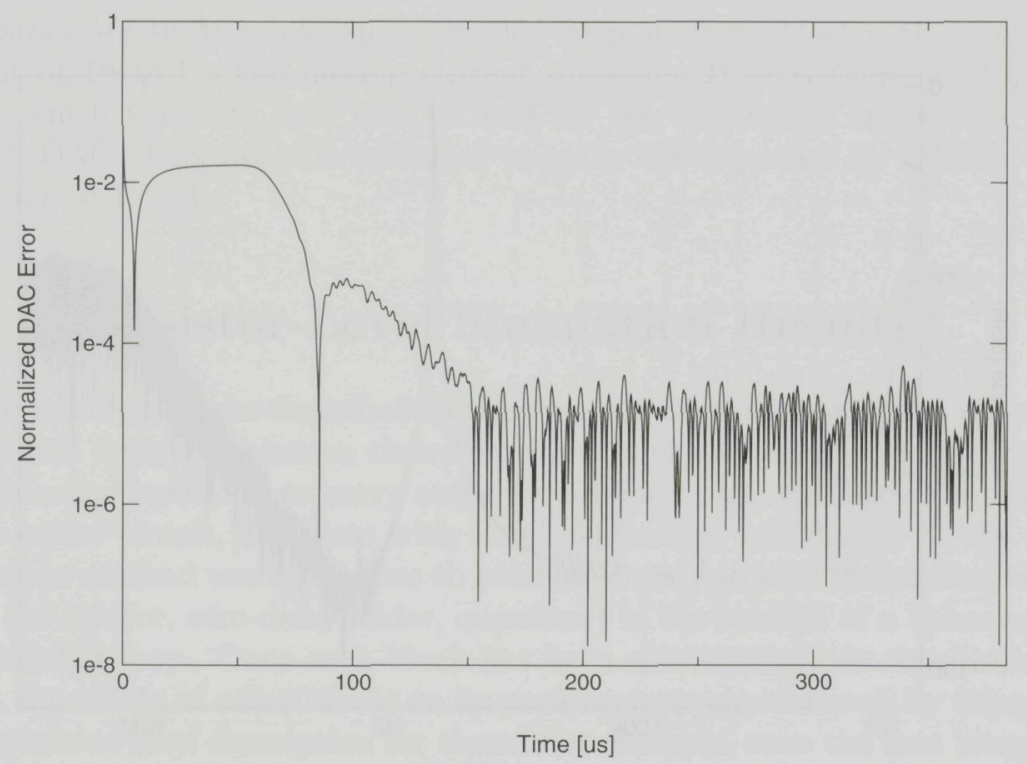

Figure 7.23: Calibration error extracted from transistor-level simulations of the active calibration loop 
Table 7.7: Performance Summary

\begin{tabular}{lc}
\hline Supply Voltage & $1.8 \mathrm{~V}$ \\
\hline$\Sigma \Delta$ Supply Current & $67 \mathrm{~mA}$ \\
1st Integrator Supply Current & $41.8 \mathrm{~mA}$ \\
2nd Integrator Supply Current & $10.0 \mathrm{~mA}$ \\
3rd Integrator Supply Current & $1.65 \mathrm{~mA}$ \\
4th Integrator Supply Current & $0.65 \mathrm{~mA}$ \\
Adder Supply Current & $2.1 \mathrm{~mA}$ \\
\hline Calibration Supply Current & $3 \mathrm{~mA}$ \\
\hline Total Power Consumption & $120 \mathrm{~mW}$ \\
\hline Signal Bandwidth & $2 \mathrm{MHz}$ \\
\hline OSR & 16 \\
\hline Maximum Input Signal & $1.275 \mathrm{~V} \mathrm{peak}$ \\
\hline Dynamic Range & $100 \mathrm{~dB}$ \\
\hline Peak SNDR & $97 \mathrm{~dB}$ \\
\hline Technology & $0.18 \mu \mathrm{m} \mathrm{CMOS} 4 \mathrm{M}$ \\
\hline Estimated Die Area & $5 \mathrm{~mm}^{2}$ \\
\hline
\end{tabular}

based on technology parameters, is also given. At a supply voltage of $1.8 \mathrm{~V}$, the main $\Sigma \Delta$ ADC is supplied with $67 \mathrm{~mA}$, more than $41 \mathrm{~mA}$ being drawn by the first integrator. The second integrator needs relatively large power because is a SC design and the OSR is only 16 so the loop gain offered by the first integrator is not very large. The third and fourth integrators have relaxed specs, therefore their power consumption is small. The zero-delay adder needs $2.1 \mathrm{~mA}$ to hold a steady signal at the input of the quantizer when all 31 comparators switch. The calibration circuitry only draws $3 \mathrm{~mA}$. Estimated die area is $5 \mathrm{~mm}^{2}$, mainly occupied by the integration capacitors and the DAC of the first integrator. 


\section{References}

[1] C. Toumazou, G. Moschytz, B. Gilbert, Trade-Offs in Analog Circuit Design, Kluwer Academic Publishers, 2002.

[2] K. Vleugels, S. Rabii, B.A. Wooley, A $2.5 \mathrm{~V}$ Broadband Multi-Bit $\Sigma \Delta$ Modulator with 95dB Dynamic Range, ISSCC 2001 Digest of Technical Papers, Feb. 2001.

[3] J. Cherry, W.M. Snelgrove, Continuous-Time Delta-Sigma Modulators for High-Speed A/D Conversion, Kluwer Academic Publishers, 2000.

[4] F. Medeiro, B.P. Verdu, A.R. Vasquez, Top-Down Design of HighPerformance Sigma-Delta Modulators, Kluwer Academic Publishers, 1999.

[5] A. Marques, High Speed CMOS Data Converters, PhD Dissertation, Katholieke Universiteit Leuven, Belgium, 1999.

[6] ***, Proceedings of the 10th Workshop on Advances in Analog Circuit Design, AACD 2001, Noordwijk, The Netherlands.

[7] J.H. Huijsing, Operational Amplifiers. Theory and Design, Kluwer Academic Publishers, 2001.

[8] A.M. Abo, P.R. Gray, A 1.5-V, 10-bit, 14.3-MS/s CMOS Pipeline Analogto-Digital Converter, IEEE Journal of Solid-State Circuits, Vol. 34, No. 5, May 1999.

[9] D.W.J. Groeneveld, et.al., A Self-Calibrated Technique for Monolithic HighResolution D/A Converters, IEEE Journal of Solid-State Circuits, Vol. 24, No. 6, December 1989.

[10] S.R. Norsworthy, R. Schreier, G.C. Temes, Delta-Sigma Data Converters, IEEE Press, 1997. 


\section{Summary}

This thesis describes the issues related to $\Sigma \Delta$ analog-to-digital converter (ADC) design in a systematic manner, from the top level of abstraction represented by the filters defining signal and noise transfer functions (STF, NTF), passing through the architecture-level where topology-related performance is calculated and simulated, and finally down to parameters of circuit elements like resistors and capacitors in the integrators and amplifier transconductances. The systematic approach allows the evaluation of different loop filters (order, aggressiveness, discrete-time or continuous-time implementation) with quantizers varying in resolution. Topologies explored can be single loops with only distributed feedforwards up to multiple cascaded loops with complex structures including more feedbacks and feedforwards. For differential circuits, with switched-capacitor integrators for discrete-time (DT) loop filters and active-RC for continuous-time (CT) ones, the passive integrator components can be calculated and the power consumption can be estimated, based on top-level requirements like harmonic distortion and noise budget.

In Chapter 1, an introduction to oversampled, noise-shaping converters is given. Starting from Nyquist-rate ADCs, it is shown that one convenient method to improve conversion resolution without increasing quantizer complexity is to over-sample the analog input signal so only a part of the quantization noise falls inside the input signal bandwidth. This can be accomplished provided the quantizer is much faster than the Nyquist frequency associated with the input signal bandwidth. To further improve conversion resolution for a given over-sampling ratio (OSR), the oversampled noise-shaping ADC is introduced that, while over-sampling the input signal, also filters the quantization noise introduced by the quantizer so only a small fraction of its power is still present inside the input signal bandwidth. Chapter 1 also describes the motivation of writing this thesis and contains an overview of the chapters.

In Chapter 2 the linear model of $\Sigma \Delta$ ADC general topology is used to introduce the expressions for NTF and STF. With integrators in the loop, NTF results in a high-pass filter and pushes most of the quantization noise power at high frequencies, while STF is a low-pass filter leaving the input signal un- 
attenuated at low frequencies. The linear model only holds if the two signals filtered by the NTF (quantization noise) and STF (input signal) respectively are independent of each other. This is not true for large input signals, so in practice the $\Sigma \Delta$ loop overloads at an input level below full-scale reference, the overloading level (OVL). The overloading is associated with modulation of the quantization noise by the input signal. With the linear model not holding for large input signals, the conversion performance must be measured based on time-domain simulations, which model the behavior of the $\Sigma \Delta$ ADC more accurately.

Discrete-time (DT) circuits are the first choice in designing the loop filter of a $\Sigma \Delta$ ADC since they fit perfectly in the sampled environment built by the sampled quantizer and DAC. With a DT loop filter, the NTF should be causal and have a unitary initial impulse response, so a connection arises between its cutoff frequency and peak magnitude near $f_{s} / 2$. More aggressive filters (with high cutoff frequency) always show a higher peak magnitude than less aggressive ones. This effect causes a reduction in the OVL. A trade-off appears between attainable DR, larger for more aggressive NTFs, and the effective number of bits (ENOB) reduced by a lower OVL. The trade-off is more important in singleloop topologies, where the two are tightly connected. In cascaded designs the connection is partially broken and the OVL is only influenced by the (low-order, high-OVL) first loop.

For single-loop topologies, an example of NTF-to-topology mapping is given. It is important to correctly size the loop coefficients which are not determined by the mapping, based on time-domain simulations. In connection with circuitlevel non-idealities, attenuation from the input of the loop of each individual integrator's noise and distortion is calculated. The DR degradation effect induced by the loop coefficient mismatch is found to be easily concealed by the circuit white noise if it dominates the quantization noise power inside the signal bandwidth. For cascaded topologies, the digital cancellation of lower-order shaped quantization noise has a major role in attaining a high DR while keeping the OVL also high. Usually approximating each cascaded loop's NTF by a scaled version of an ideal DT high-pass filter, the digital noise cancellation is highly sensitive to loop coefficient mismatch.

The CT loops filters are built starting from a DT NTF/STF prototype. The sampled nature of the quantizer along with the (high) OSR which makes the input signal to be slow varying during one clock cycle allows for a mapping from DT domain to CT domain based on identical impulse response of NTF and STF. DAC waveform affects this mapping however, with intersymbol interference introducing more in-band quantization noise power. Clock jitter is also a modulation of DAC waveform but its main contribution is direct addition of white noise power inside the signal bandwidth. Direct NTF and STF mapping opens the possibility of comparison between DT and CT loop filter performance in terms of OVL offered on the same topology with identical aggressiveness. CT 
loop filters are not only sensitive to coefficient mismatch but also to initial accuracy of the coefficients which is likely to be up to $30 \%$ in CMOS processes. This constraint has to be taken into account when the loop coefficients are designed. Cascaded topologies with CT loop filters can be implemented without adding custom-designed digital cancellation filters if a sampler is introduced between two consecutive loops, so all loops process DT input signals. There is need a for calibration of digital coefficients to cope with initial coefficient accuracy.

In Chapter 3 different DT integrator topologies are presented and one of them, the switched-capacitor (SC) integrator is further analyzed as being the most linear option. Multiple DACs give multiple topologies for the summing $\mathrm{SC}$ integrators to be used in $\Sigma \Delta$ ADCs. Circuit non-idealities like finite and non-linear opamp gain, finite opamp bandwidth and slew-rate and non-zero on-resistance of MOS switches introduce errors in the transfer function of the integrator, degrading conversion resolution and total harmonic distortion. From calculations or simulations, lower or upper limits can be derived form these nonidealities so the target conversion performance is reached. In a switched circuit, it is also important to correctly time and apply the clock phases to the different switches to reduce additional non-ideal effects causing harmonic distortion.

The power consumption of a SC integrator is estimated based on its noise budget and total harmonic distortion requirements. The switched capacitors are calculated from the noise budget and they are setting the speed (transconductance $g_{m}$ ) requirements for the opamp used to drive them. A compact expression for opamp's $g_{m}$ can be derived if the opamp's input transistors are operated in weak inversion.

Other discrete-time blocks used in DT $\Sigma \Delta$ ADC implementations include SC zero-delay amplifiers and dynamic quantizers. Multibit quantizers can be build taking care the strong kick-back does not affect the reference levels derived from one reference voltage by a resistive string. With multibit quantizers, multibit DACs are also implemented by splitting the DAC capacitor into many unit DAC capacitors, all switched between two reference voltages and the inputs of the opamp. Correction of unit capacitors mismatch is difficult so usually dynamic element matching is employed to spread the effect of the errors in the entire sampled bandwidth.

In Chapter 4 the circuits used to implement CT loop filters are explained. From different methods to implement the integrators, the active- $\mathrm{RC}$ is preferred for its enhanced linearity due to the local feedback loop closed over a high-gain opamp. Three types of DAC generate three different topologies for the summing $\mathrm{CT}$ integrators with active- $\mathrm{RC}$ circuits, each of them with their advantages. The finite and non-linear opamp $g_{m}$ is found to be the most important non-ideality, increasing total harmonic distortion. It is not difficult to design the opamp so the slewing does not occur in normal operation and the opamp unity-gain bandwidth exceeds the limit where the integrator transfer function is affected. The voltage-to-current conversion resistors and the integration capacitor are 
calculated from the noise budget and the $\Sigma \Delta$ loop coefficients. The power consumption is estimated from the $g_{m}$ of the opamp, calculated based on distortion requirements and resistor values.

The quantizers used in CT $\Sigma \Delta$ ADCs with non-return-to-zero (NRZ) DACs must switch fast to reduce excess loop delay. Excess loop delay affects the NTF implemented by the loop and reduces the DR by increasing the in-band quantization noise power. If return-to-zero (RTZ) DACs are used the speed restriction on the quantizer is greatly reduced since more decision time is available. To implement RTZ functionality, the current-mode DACs need an extra current path where they are connected during RTZ to avoid saturation of the current sources. One advantage of using current-mode DACs in multibit designs is the ease of background calibration to reduce DAC cell mismatch.

In Chapter 5 the algorithms used to implement an exhaustive search in the $\Sigma \Delta$ ADC design space are explained. The search is conducted at two abstraction levels. Firstly, the filter-level design uses the linear model of $\Sigma \Delta$ ADCs to estimate the DR performance, and use this to reject a major part of the possible solutions. The feasibility of analyzed solutions is decided based on a database containing, for different loop orders and number of quantizer bits, the ranges of usable NTF aggressiveness as expressed by the peak magnitude of the DT variant. The cascaded solutions are analyzed as extremely aggressive yet feasible single-loop solutions. The solutions passing the filter-level evaluations are processed at architecture level to extract their final performance from long time-domain simulations. For this, according to each case, DT to CT conversion of NTF and STF is performed and/or single-loop to cascade mapping. The loop coefficients are calculated to bound the integrator outputs within required limits so clipping only occurs when the input signal reaches the OVL. The DT loops are found to be designable in a wider NTF aggressiveness range than their CT equivalents. The power consumption of each solution passing the performance tests is estimated, and only a "top ten" of solutions are passed to yield estimation and optimization in a long Monte-Carlo simulation step. The performance of this exhaustive search is proven by its ability to find previously published, state-of-the-art solutions among the "top-ten" generated for the corresponding targets in terms of DR, signal bandwidth and supply voltage.

In Chapter 6 , the design of a $\Sigma \Delta \mathrm{ADC}$ for low-voltage, mobile telephony applications is explained in detail, from NTF/STF design at filter level down to transistor level. This is a custom-made design, developed to replace both the preamplifier and the ADC normally used to convert the output signal of an electret microphone. To connect directly to the microphone, a CT first integrator has been designed which has high input impedance, has single-ended input to the microphone side and differential output to connect to the rest of the $\Sigma \Delta$ loop filter, and can also integrate a differential DAC current according to the quantizer decision. The topology is a fourth-order, single-loop, single-bit which attains $80 \mathrm{~dB}$ white-noise limited DR in a bandwidth of $11 \mathrm{kHz}$. Supply 
voltage is $1.8 \mathrm{~V}$, which for the chip implemented in $0.5 \mu \mathrm{m}$ CMOS means less than two threshold voltages. The supply current is $600 \mu \mathrm{A}$ for the $\Sigma \Delta$ ADC and $350 \mu \mathrm{A}$ for the on-chip voltage reference. Amplitude of the input signal as supplied by the microphone is $125 \mathrm{mV}$, centered around the ground node. Peak SNDR is $62 \mathrm{~dB}$, limited by distortion introduced by the first integrator, caused by the single-ended to differential conversion.

In Chapter 7 , the design of a broadband $\Sigma \Delta$ ADC for low-voltage, lowpower wire-line communications is presented. The $\Sigma \Delta$ ADC loop, as generated by automated, designer-constrained search using the algorithms described in Chapter 5, is a fourth-order single-loop with 5-bit quantizer. 100dB whitenoise limited DR is attained. To reduce power consumption and chip area, the first integrator is implemented with a CT active-RC topology. The rest of the loop maintains DT functioning to take advantage of better stability at highly aggressive NTF. The circuit is designed in $0.18 \mu \mathrm{m}$ digital CMOS technology with only 4 metal layers. The supply voltage is $1.8 \mathrm{~V}$ and the transistor-level (simulated) power consumption is $120 \mathrm{~mW}$. The simulated peak SNDR is $97 \mathrm{~dB}$, attained by calibrating the multibit DAC in the first integrator in an active, background calibration scheme containing a second $\Sigma \Delta$ ADC which uses each of the DAC cells as a one-bit DAC on each calibration slot. 


\section{Samenvatting}

Dit proefschrift beschrijft de onderwerpen die samenhangen met het ontwerp van de $\Sigma \Delta$ analoog-naar-digitaalomzetter (ADC) op een systematische manier, van het hoogste abstractieniveau vertegenwoordigd door filters die de signaalen ruisoverdrachtsfuncties (STF, NTF) definiëeren, via het architectuurniveau, waar de prestatie gerelateerd aan de topologie wordt berekend en gesimuleerd, uiteindelijk naar het niveau van de parameters van circuitelementen zoals weerstanden en condensatoren in de integrators, en de versterkertransconductanties. Deze systematische aanpak maakt het mogelijk om verschillende soorten lusfilters te vergelijken (ordegrootte, agressiviteit, tijddiscrete of tijdcontinue implementatie) met quantisatoren van verschillende resoluties. De onderzochte topologieën kunnen bestaan uit één enkele lus met alleen gedistribueerde vooruitkoppeling tot meervoudig gecascadeerde lussen met complexe structuren die meerdere vooruitkoppelingen en terugkoppelingen bevatten. Voor differentiale circuits, met geschakelde-condensator integrators voor tijddiscrete lusfilters en actieve-RC voor tijdcontinue lusfilters kunnen de passieve integrator-elementen worden berekend en het vermogensverbruik worden geschat, op basis van eisen zoals de toegestane harmonische vervorming en ruis.

In Hoofdstuk 1 wordt een inleiding tot overgesamplede ruisverdelingsomzetters gegeven. Beginnend bij de Nyquist-snelheid ADC's wordt aangetoond dat één praktische methode om de conversieresolutie te verbeteren zonder de complexiteit van de quantisator te vergroten het oversampelen van het analoge ingangssignaal is, zodat maar een deel van de kwantisatieruis binnen de bandbreedte van het ingangssignaal valt. Dit kan worden gedaan als de quantisator veel sneller is dan de Nyquistfrequentie die met de bandbreedte van het ingangssignaal wordt geassocieerd. Om de conversieresolutie voor een gegeven oversamplingverhouding (OSR) verder te verbeteren wordt de overgesamplede ruisverdeling $\mathrm{ADC}$ geintroduceerd, welke terwijl hij het ingangssignaal oversampled, ook de kwantisatieruis filtert die de quantisator introduceert, zodat er nog slechts een fractie van het vermogen aanwezig blijft binnen de ingangssignaalbandbreedte. Hoofdstuk 1 motiveert ook waarom dit proefschrift werd geschreven en bevat een overzicht van de hoofdstukken.

In Hoofdstuk 2 wordt het lineaire model van de algemene topologie van de 
$\Sigma \Delta$ ADC gebruikt om uitdrukkingen voor de NTF en STF te introduceren. Met integrators in de lus resulteert NTF in een hoogdoorlaatfilter die het grootste deel van de kwantisatieruis naar hoge frequenties duwt, terwijl STF een laagdoorlaatfilter is die het ingangssignaal niet dempt bij lage frequenties. Het lineaire model geldt alleen als de twee signalen die door de NTF (kwantisatieruis) en STF (ingangssignaal) worden gefilterd onafhankelijk zijn van elkaar. Dit gaat niet op voor grote ingangssignalen, dus in de praktijk overstuurt de $\Sigma \Delta$ lus bij een ingangsniveau dat lager ligt dan het maximale referentiesignaal, het oversturingsniveau (OVL). Oversturing wordt geassocieerd met modulatie van de kwantisatieruis door het ingangssignaal. Omdat het lineaire model niet geldt voor grote ingangssignalen moet de conversieprestatie worden gemeten op basis van simulaties in het tijddomein, welke het gedrag van de $\Sigma \Delta$ ADC nauwkeuriger kunnen modelleren.

Tijddiscrete (DT) circuits zijn de eerste keus bij het ontwerpen van een lusfilter van een $\Sigma \Delta A D C$ omdat ze perfect passen in de gesamplede omgeving opgebouwd uit de gesamplede quantizer en DAC. Met een DT lusfilter, zou de NTF causaal moeten zijn en een initiële impulsrespons van 1 moeten hebben, zodat er een verbinding ontstaat tussen zijn kantelfrequentie en piekhoogte van nabij fs/2. Agressievere filters (die een hogere kantelfrequentie hanteren) laten altijd hogere pieken zien dan minder agressieve filters. Dit effect zorgt voor een vermindering van de OVL. Hierdoor moet een afweging worden gemaakt tussen de haalbare DR, welke groter is voor agressievere NTF's, en het effectieve aantal bits (ENOB), welke wordt verkleind door een lagere OVL. Het maken van deze afweging is belangrijker voor enkele-lus-topologieën, waar beide factoren naww samenhangen. In cascade-ontwerpen valt de samenhang gedeeltelijk weg en wordt de OVL alleen beinvloed door de (lage-orde hoge-OVL) eerste lus.

Voor enkele-lus-topologieën geven we een voorbeeld van NTF-naar-topologie mapping. Het is belangrijk om de luscoëfficiënten die niet door de mapping worden bepaald correct te schalen op basis van simulaties in het tijddomein. In verband met niet-idealiteiten op circuitniveau wordt de vezwakking van de ruis en vervorming van iedere integrator naar de ingang van de lus berekend. Het DR degradatie-effect dat wordt veroorzaakt door de mismatch van de luscoëfficiënt blijkt makkelijk verborgen te worden door de witte ruis in het circuit als deze de kwantisatieruis binnen de signaalbandbreedte overheerst. Voor cascade-topologieën speelt het digitaal onderdrukken van lagere-orde gevormd kwantisatieruis een grote rol in het verkrijgen van een hoge DR terwijl men de OVL ook hoog houdt. Omdat de digitale onderdrukking van ruis over het algemeen de NTF van elke cascadelus benadert met een geschaalde versie van een ideale DT hoogdoorlaatfilter is deze erg gevoelig voor foutieve aanpassing van de luscoëfficiënt.

De CT lusfilters zijn gebouwd op basis van een DT NTF/STF prototype. De gesamplede aard van de quantisator samen met de (hoge) OSR welke ervoor zorgt dat het ingangssignaal langzaam varieert gedurende één klokcyclus maakt 
een mapping van het DT domein naar het CT domein op basis van een identieke impulsrespons van NTF en STF mogelijk. De DAC golfvorm beinvloedt deze mapping echter, waardoor signaalinterferentie meer kwantisatieruis introduceert binnen de band. Klok-jitter is ook een modulatie van de DAC golfvorm maar de belangrijkste bijdrage hiervan is de directe toevoeging van witte ruis binnen de signaalbandbreedte. Directe NTF en STF mapping maakt het mogelijk om de prestatie van de DT en CT lusfilter te vergelijken met betrekking tot de OVL die deze bieden voor dezelfde topologie voor even agressieve filters. CT lusfilters zijn niet alleen gevoelig voor foutieve aanpassing van de luscoëfficiënt, maar ook voor de initiële nauwkeurigheid van de coëfficiënten; meestal is dit $30 \%$ in CMOS processen. Met deze beperking moet rekening worden gehouden in het ontwerp van de luscoëfficiënten. Cascade-topologiën met CT lusfilters kunnen worden toegepast zonder de toevoeging van specifiek ontworpen digitale ruisonderdrukkers als er een sampler wordt geplaatst tussen twee opeenvolgende lussen zodat alle lussen DT ingangssignalen verwerken. Kalibratie van de digitale coëfficiënten is nodig om de initiële onnauwkeurigheid te compenseren.

In Hoofdstuk 3 worden verschillende DT integrator-topologieën beschreven en één van deze, de geschakelde-condensator (SC) integrator wordt verder geanalyseerd omdat deze het meest lineair is. Het gebruik van meerdere DAC's resulteert in meerdere topologieën voor de sommerende SC integrators die kunnen worden gebruikt in $\Sigma \Delta$ ADC's. Circuit niet-idealiteiten zoals een eindige en niet-lineaire opampversterking, een eindige opampbandbreedte en slewrate en de aan-weerstand van MOS-schakelaars introduceren fouten in de overdrachtsfunctie van de integrator, verslechteren de conversieresolutie en de totale harmonische vervorming.

Het vermogenverbruik van een SC integrator wordt geschat op basis van hoeveel ruis er aanwezig mag zijn en de eisen t.a.v. de totale harmonische vervorming. De geschakelde condensatoren worden berekend op basis van de hoeveelheid toegestane ruis en zij bepalen de vereiste snelheid (transconductantie $g_{m}$ ) van de operationele versterker die wordt gebruikt om ze aan te sturen. Een compacte uitdrukking voor de $g_{m}$ van de operationele versterker kan worden afgeleid als de ingangstransistoren die worden gebruikt ingesteld zijn in zwakke inversie.

Andere tijddiscrete systemen die in DT $\Sigma \Delta$ ADC toepassingen worden gebruikt zijn versterkers zonder vertraging en dynamische quantisatoren. Het is mogelijk om multi-bit quantisatoren te bouwen, waarbij ervoor gezorgd moeten worden dat de sterke terugslag de referentieniveaus niet beinvloedt, die worden afgeleid van een referentiespanning door middel van een weerstands string. Bij multi-bit quantizers worden ook multi-bit DAC's toegepast waarvan de DAC condensator is opgesplitst in verschillende units, die allemaal tussen twee referentiespanningen zijn geschakeld en de inputs van de operationele versterker. Het is moeilijk om een mismatch tussen condensator units te corrigeren, dus meestal wordt dynamic element matching gebruikt om het effect van de fouten 
over de gehele bandbreedte te verdelen.

In Hoofdstuk 4 wordt uitleg gegeven van de circuits die worden gebruikt om de CT lusfilters toe te passen. Van de verschillende methoden die worden gebruikt voor het toepassen van de integrators wordt de voorkeur gegeven aan de actieve $\mathrm{RC}$ vanwege zijn verbeterde lineariteit die wordt veroorzaakt door een lokale terugkoppeling lus geschakeld over een operationele versterker met hoge versterking. Drie soorten DAC's genereren drie verschillende topologieën voor de sommerende CT integrators met actieve RC circuits, waarvan elk zijn eigen voordelen heeft. De eindige en niet-lineaire operationele $g_{m}$ van de verterker blijkt de belangrijkste niet-idealiteit, die de totale harmonische vervorming doet toenemen. Het is niet moeilijk om de opamp zo te ontwerpen dat slewing niet optreedt bij normale werking en de unity-gain bandbreedte boven de grens ligt waar de overdrachtsfunctie van de integrator wordt beinvloed. De weerstanden voor het omzetten van spanning in stroom en de integratie-condensator worden berekend op basis van de hoeveelheid toegestane ruis en de $\Sigma \Delta$ luscoëfficiënten. Het vermogensverbruik wordt geschat op basis van de gm van de operationele versterker, welke wordt berekend op basis van de vervormingvereisten en de weerstandswarden.

De quantisatoren die worden gebruikt in CT $\Sigma \Delta$ ADC's met DAC's die de stroom niet terug brengen tot nul ('non-return-to-zero' ofwel NRZ DAC's) moeten snel schakelen om overmatige vertraging in de lus te beperken. Overmatige vertraging in de lus beinvloedt de NTF die door de lus wordt geimplementeered en vermindert de DR door het vergroten van de kwantiesatieruis binnen de band. Als DAC's worden gebruikt die de stroom wel terug brengen naar nul ('return-to-zero' ofwel RTZ DAC's) wordt de beperking op de snelheid van de quantisator sterk gereduceerd omdat meer beslistijd voorhanden is. Om de RTZ functionaliteit toe te passen moet er voor een extra stroompad in de stroomgestuurde DAC's worden gezorgd waaraan de stroombronnen worden verbonden tijdens RTZ om verzadiging te vermijden. Een voordeel van het gebruik van stroombestuurde DAC's in multi-bit ontwerpen is het gemak waarmee deze op de achtergrond kunnen worden gekalibreerd om de DAC cel mismatch te verminderen.

In Hoofdstuk 5 worden de algoritmes beschreven die worden gebruikt voor een uitputtend onderzoek van de $\Sigma \Delta$ ADC ontwerpruimte. Dit onderzoek wordt uitgevoerd op twee abstractieniveau's. Allereerst wordt op het filter ontwerpniveau het lineaire model van $\Sigma \Delta$ ADC's gebruikt om de DR prestatie te schatten, en gebruiken deze om een groot deel van de mogelijke oplossingen uit te sluiten. De haalbaarheid van de geanalyseerde oplossingen wordt beoordeeld op basis van een database die de bereiken van bruikbare NTF agressiviteit als uitgedrukt door de piekhoogte van de DT variant voor verschillende lus-ordegrootten en aantal quantisatie bits bevat. De oplossingen voor gecascadeerde topologieën worden geanalyseerd als zijnde extreem agressieve maar haalbare enkele-lus oplossingen. De oplossingen die goed uit de filter-niveaubeoordeling komen wor- 
den op architectuurniveau verwerkt om hun uiteindelijke prestatie te verkrijgen uit lange simulaties in het tijd-domein. Voor elke van deze gevallen wordt DT naar CT conversie van NTF en STF uitgevoerd en/of enkele-lus naar cascade mapping. De luscoëfficiënten worden berekend om de integratoruitgangssignalen te begrenzen binnen de toegestane limieten zodat amplitudebegrenzing alleen wordt uitgevoerd als het ingangssignaal de OVL bereikt. Het bleek dat DT lussen kunnen worden ontworpen met een groter agressiviteitbereik dan hun CT equivalenten. Het vermogensverbruik van elke oplossing die goed uit de prestatietests komt is geschat, en alleen een top tien van oplossingen zijn gebruikt voor het schatten en optimalisatie in een lange Monte-Carlo simulatiestap. De prestatie van dit uitputtend onderzoek wordt aangetoond door haar vermogen om de allernieuwste eerder gepubliceerde oplossingen te vinden binnen de top tien die werd gegenereerd voor de overeenkomstige doelen met betrekking tot de DR, de signaalbandbreedte en de voedingsspanning.

In Hoofdstuk 6 wordt het ontwerp van een $\Sigma \Delta$ ADC voor mobiele telefonietoepassingen die op lage spanning werken uitvoerig uitgelegd, van het NTF/STF ontwerp op filterniveau tot op het transistorniveau. Dit is een op maat gemaakt ontwerp, welke is ontwikkeld om zowel de voorversterker en de ADC die normaliter wordt gebruikt om het uitgangssignaal van een electret microfoon te vervangen. Om de microfoon rechtstreeks op de ADC aan te kunnen sluiten is een CT eerste integrator ontworpen die een hoge ingangsimpedantie heeft, een single-ended ingang aan de microfoonkant en een differentiële ingang om de rest van de $\Sigma \Delta$ lusfilter aan te sluiten, en kan ook een differentiële DAC stroom integreren op basis van de beslissing van de quantisator. De topologie is een vierde-orde enkele-lus één-bits topologie die $80 \mathrm{~dB}$ DR bereikt (door witte ruis beperkt) in een bandbreedte van $11 \mathrm{kHz}$. De voedingsspanning is $1.8 \mathrm{~V}$, wat voor de chip die wordt toegepast in een $0.5 \mu \mathrm{m}$ CMOS minder dan twee drempelspanningen betekent. De voedingsstroom is $600 \mu \mathrm{A}$ voor de $\Sigma \Delta \mathrm{ADC}$ en $350 \mu \mathrm{A}$ voor de spanningsreferentie op de chip. De amplitude van het ingangssignaal zoals afkomstig van de microfoon is $125 \mathrm{mV}$, gecentreerd om het aardpunt. De piek SDNR is $62 \mathrm{~dB}$, beperkt door vervorming geintroduceerd door de eerste integrator, veroorzaakt door de conversie van single-ended naar differentieel.

In Hoofdstuk 7 wordt het ontwerp van een breedband $\Sigma \Delta$ ADC voor lagespanning laag-vermogen 'wire-line communications' gegeven. De $\Sigma \Delta$ ADC lus, zoals gegenereerd door een geautomatiseerd onderzoek gestuurd door de ontwerper met gebruik van de algoritmen uit Hoofdstuk 5 is een vierde-orde enkele-lus topologie met 5 bits quantisator. Een DR van 100dB (beperkt door witteruis) werd verkregen. Om het vermogensverbruik en de benodigde chipoppervlakte te verkleinen is de eerste integrator toegepast met een CT-actieve RC topologie. De rest van de lus behoudt DT werking om gebruik te maken van de betere stabiliteit bij een agressievere NTF. Het circuit is ontworpen in $0.18 \mathrm{~m}$ digitale CMOS technologie met slechts 4 metalen lagen. De voedingsspanning is $1.8 \mathrm{~V}$ 
en het vermogensverbruik gesimuleerd op transistorniveau is $120 \mathrm{~mW}$. De gesimuleerde piek SNDR is $97 \mathrm{~dB}$, verkregen door kalibratie van de multi-bit DAC in de eerste integrator in een actieve achtergrondkalibratieschema die een tweede $\Sigma \Delta \mathrm{ADC}$ bevat die ieder van de DAC cellen gebruikt als een één-bits DAC voor iedere kalibratie moment. 


\section{Acknowledgments}

The thesis so far has been about the author and his work. But all the work presented here, and the thesis itself, would not have been possible without the support and understanding of many people. This Chapter belongs to them.

First of all, I would like to thank to Professor Johan Huijsing for trusting me with a PhD project. He has been supportive without limiting my freedom in choosing the research direction. He taught me many important lessons, and not only about electronics. I remember one day when he told me "Think of me as your team-mate". And that he was, for all these years.

I wish to thank to Professor Mircea Bodea from the Laboratory of Microelectronic Systems (LSmE) in Bucharest who helped me decide to start my $\mathrm{PhD}$ work at T.U.Delft, knowing this was bad for his Laboratory but good for me. The "founders" of LSmE are still some of my best friends: Dr. Claudius "Claus" Dan, Dr. Sorin Spanoche and Dr. Serban Popescu.

Special thanks go to Dr. Lucien Breems from Philips Research, for helping me understand more about $\Sigma \Delta \mathrm{ADC}$ design than anybody else and for actively supporting me in the last phase of this $\mathrm{PhD}$ work. He was also a great office mate during his years at T.U.Delft. I thank Dr. Marcel Pelgrom from Philips Research for helping me produce a chip in a performant CMOS technology. At Philips Design Center in Delft, Dr. Anton Bakker provided financial and administrative support; Gian Hoogzaad and Marto-Jan Koerts answered my technical questions and taught me table tennis.

I would like to thank to my office mates Svetla Matova and Ourang AkhzarMehr for a great work environment. Special thanks go to Paolo Silva for a thorough review of this thesis and interesting technical and non-technical discussions.

I would also like to thank to the people at National Semiconductors Design Center in Delft, Dr. Ruud Eschauzier, Arie van Rhijn and Nico van Rijn for pro- 
viding the target and the means to design and measure the digital microphone chip.

I thank to the people at Electronic Instrumentation Laboratory for the pleasant environment, good technical discussions and great day-out parties. I would like to mention Edmond Cretu, who first introduced me to this group.

Last but not least, I want to thank to my wife Simona Maria, for understanding and supporting me during long working hours and weekends. To my parents, who taught me what was good and wrong, and knew when to let me decide for myself. To my sister Gabriela who always supports my decisions. 


\section{List of Papers}

O. Bajdechi, J.H. Huijsing,

A $1.8 \mathrm{~V}$ Delta-Sigma Modulator Interface for Electret Microphone with On-Chip Reference,

IEEE Conference on Custom Integrated Circuits, 2001.

M.F. Snoeij, O. Bajdechi, J.H. Huijsing,

$A$ 4th-order Switched-Capacitor Sigma-Delta A/D Converter Using a High-Ripple Chebyshev Loop Filter,

The 2001 IEEE International Symposium on Circuits and Systems, 2001.

O. Bajdechi, J.H. Huijsing,

A 1.8 V Delta-Sigma Modulator Interface for an Electret Microphone with On-Chip Reference,

IEEE Journal of Solid-State Circuits, Vol. 37, Issue 3, March 2002.

O. Bajdechi, J.H. Huijsing, G. Gielen,

Fast Exploration Delta-Sigma ADC Design Space,

The 2002 IEEE International Symposium on Circuits and Systems, 2002.

O. Bajdechi, J.H. Huijsing, G. Gielen,

Optimal Design of Delta-Sigma ADCs by Design Space Exploration, 39th Design Automation Conference, 2002.

O. Bajdechi, J.H. Huijsing, G. Gielen, Power Optimization in Sigma-Delta ADC Design,

14th International Conference on Digital Signal Processing, 2002. 